\title{
Dynamics Control Approaches to Improve Vibratory Environment of the Helicopter Aircrew
}

by

Viresh Kanchana Wickramasinghe, M. Sc., P. Eng.

A thesis submitted to the Faculty of Graduate and Postdoctoral Affairs in partial fulfillment of the requirements

for the degree of

\section{Doctor of Philosophy}

Ottawa-Carleton Institute for Mechanical and Aerospace Engineering

Department of Mechanical and Aerospace Engineering

Carleton University

Ottawa, Ontario

December 2012

(C) Copyright by Viresh Kanchana Wickramasinghe, 2012 
Library and Archives

Canada

Published Heritage

Branch

395 Wellington Street

Ottawa ON K1A ON4

Canada
Bibliothèque et

Archives Canada

Direction du

Patrimoine de l'édition

395 , rue Wellington

Ottawa ON K1A ON4

Canada
Your file Votre référence

ISBN: 978-0-494-94237-6

Our file Notre référence

ISBN: $978-0-494-94237-6$
NOTICE:

The author has granted a nonexclusive license allowing Library and Archives Canada to reproduce, publish, archive, preserve, conserve, communicate to the public by telecommunication or on the Internet, loan, distrbute and sell theses worldwide, for commercial or noncommercial purposes, in microform, paper, electronic and/or any other formats.

The author retains copyright ownership and moral rights in this thesis. Neither the thesis nor substantial extracts from it may be printed or otherwise reproduced without the author's permission.
AVIS:

L'auteur a accordé une licence non exclusive permettant à la Bibliothèque et Archives Canada de reproduire, publier, archiver, sauvegarder, conserver, transmettre au public par télécommunication ou par l'Internet, prêter, distribuer et vendre des thèses partout dans le monde, à des fins commerciales ou autres, sur support microforme, papier, électronique et/ou autres formats.

L'auteur conserve la propriété du droit d'auteur et des droits moraux qui protege cette thèse. $\mathrm{Ni}$ la thèse ni des extraits substantiels de celle-ci ne doivent être imprimés ou autrement reproduits sans son autorisation.
In compliance with the Canadian Privacy Act some supporting forms may have been removed from this thesis.

While these forms may be included in the document page count, their removal does not represent any loss of content from the thesis.
Conformément à la loi canadienne sur la protection de la vie privée, quelques formulaires secondaires ont été enlevés de cette thèse.

Bien que ces formulaires aient inclus dans la pagination, il n'y aura aucun contenu manquant. 


\section{Abstract}

Although helicopter has become a versatile mode of aerial transportation, high vibration levels leads to poor ride quality for its passengers and aircrew. Undesired vibration transmitted through the helicopter seats have been known to cause fatigue and discomfort to the aircrew in the short-term as well as neck strain and back pain injuries due to longterm exposure. This research study investigated the use of novel active as well as passive methodologies integrated in helicopter seats to mitigate the aircrew exposure to high vibration levels. Due to significantly less certification effort required to modify the helicopter seat structure, application of novel technologies to the seat is more practical compared to flight critical components such as the main rotor to reduce aircrew vibration.

In particular, this research effort developed a novel adaptive seat mount approach based on active vibration control technology. This novel design that incorporated two stacked piezoelectric actuators as active struts increases the bending stiffness to avoid the low frequency resonance while generating forces to counteract higher harmonic vibration peaks. A real-time controller implemented using a feed-forward algorithm based on adaptive notches counteracted the forced vibration peaks while a robust feedback control algorithm suppressed the resonance modes. The effectiveness of the adaptive seat mount system was demonstrated through extensive closed-loop control tests on a full-scale helicopter seat using representative helicopter floor vibration profiles. Test results concluded that the proposed adaptive seat mount approach based on active control technology is a viable solution for the helicopter seat vibration control application. In addition, a unique flight test using a Bell-412 helicopter demonstrated that the aircrew is exposed to high levels of vibration during flight and that the whole body vibration spectrum varied substantially depending on operating conditions as well as the aircrew configurations. This investigation also demonstrated the suitability of integrating novel energy absorbing cushion materials to the seat as a low cost solution to improve aircrew vibration suppression. Therefore, it was recommended to pursue certification of novel seat cushion materials as a near-term solution to mitigate undesirable occupational health hazards in helicopter aircrew due to vibration exposure. 


\section{Acknowledgements}

I wish to express sincere appreciation to my thesis advisors, Professor Fred Nitzsche and Professor Jurek Sasiadek, for their support, encouragement and guidance. I am also grateful to Professor Carlos Cesnik from the University of Michigan and Professor Dan Necsulescu from the University of Ottawa for serving on my committee. I would like to thank other members of the thesis examination committee from Carleton University, namely, Professor Daniel Feszty, Professor Abhijit Sarkar and Professor Joana Torres Da Rocha, for their time in reviewing dissertation and providing valuable suggestions.

This research project could not haven possible without the support of the National Research Council Canada. In particular, I would like to thank Dr. David Zimcik, the Group Leader of the Aeroacoustics and Structural Dynamics Group, for continuous encouragement and insightful guidance. In addition, I would like to thank my colleagues, namely, Dr. Anant Grewal and Dr. Yong Chen, Dr. Sebastian Ghinet, Luc Hurtubise and Brent Lawrie for their invaluable guidance and continuous support throughout this research project. I would also like to acknowledge the support from NRC Bell-412 helicopter crew, including, Stephan Carignan, Bill Gubbles and Robert Erdos, for accommodating unusual requests with a smile in order to conduct unique flight tests.

Finally, I would like to most sincerely thank my wife, Dinu, for her patience, understanding, encouragement, and unconditional support throughout this program. I appreciate all the hugs and encouraging comments from daughter Seyla and son Seth through the years. I am also grateful to both my parents and family for their encouragement and support. 


\section{Table of Contents}

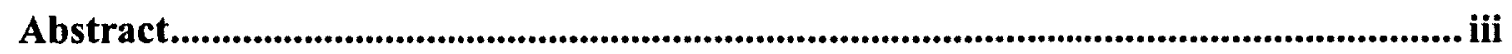

Acknowledgements ......................................................................................................................... iv

Table of Contents ....................................................................................................................... v

List of Tables ....................................................................................................................................... ix

List of Figures........................................................................................................................... $\mathrm{x}$

List of Abbreviations ..................................................................................................................... xiii

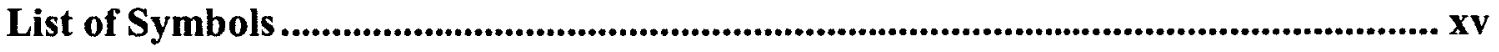

Chapter 1 Helicopter Aircrew Vibration and Control Approaches..................... 1

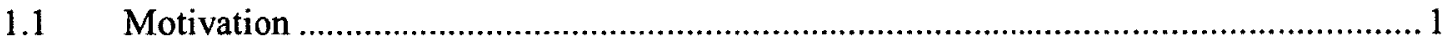

$1.2 \quad$ Background in Helicopter Vibration Control .............................................................

1.2.1 Passive Vibration Control for Helicopters ..............................................................

1.2.2 Active Rotor Control for Helicopters ..................................................................

1.2.3 Active Control of Structural Response (ACSR) ………......................................

1.3 Adaptive Helicopter Seat Mounts.............................................................................. 10

1.3.1 Current State of Adaptive Seat Mount Developments ........................................... 10

1.3.2 Active Control Technology for Adaptive Seat Mounts............................................11

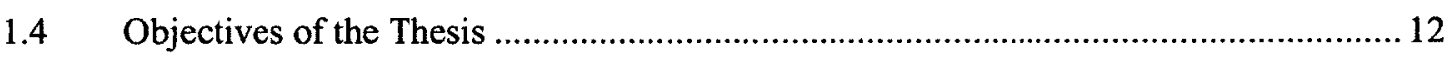

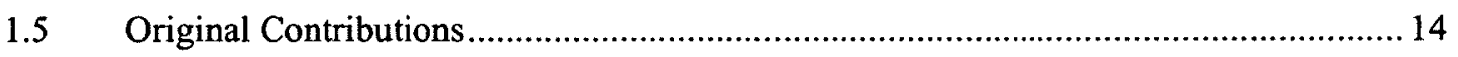

1.6 Organization of the Thesis..................................................................................... 16

Chapter 2 Flight Tests to Characterize Helicopter Aircrew Vibration ............ 17

2.1 Helicopter Seat Vibration .......................................................................... 17

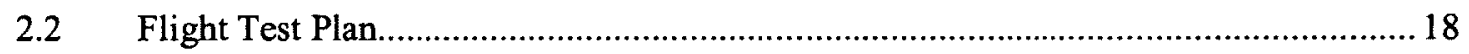

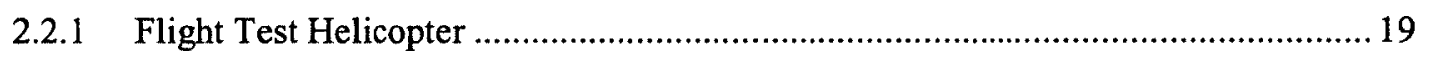

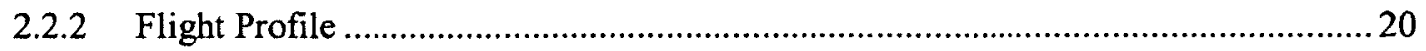

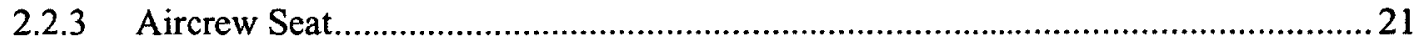

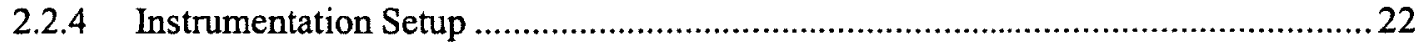

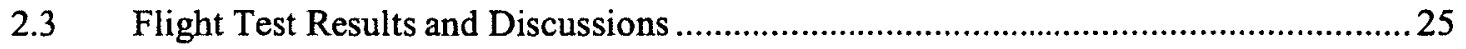




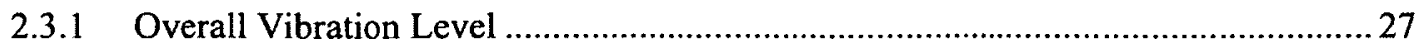

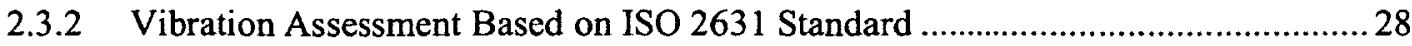

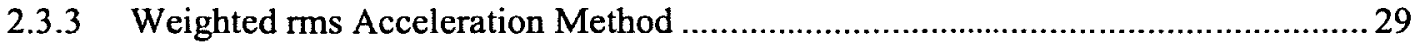

2.3.4 Maximum Transient Vibration Value …............................................................... 30

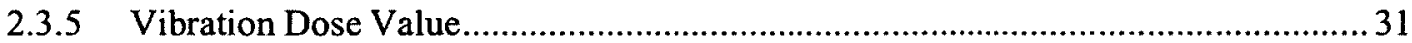

2.3.6 Assessment of Aircrew Health, Comfort and Perception......................................... 32

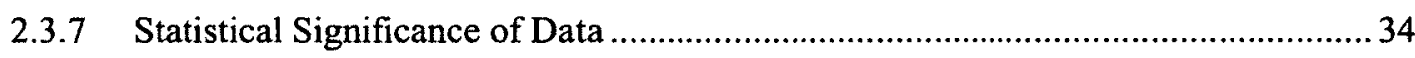

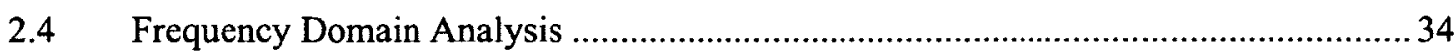

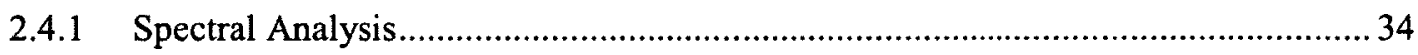

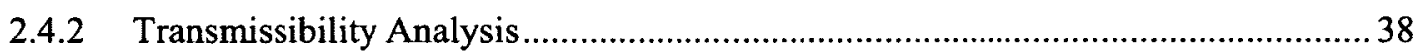

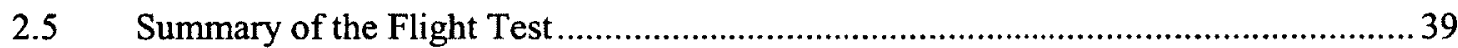

Chapter 3 Characterization of Seat System Dynamics for Simulation............ 41

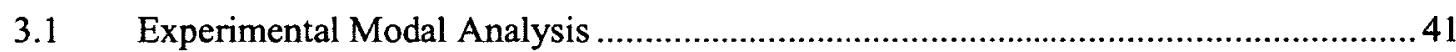

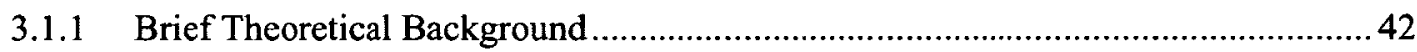

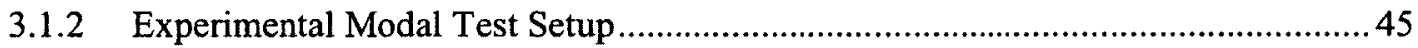

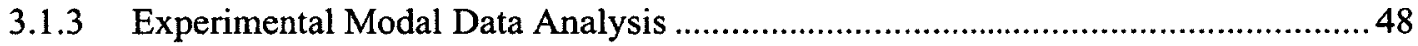

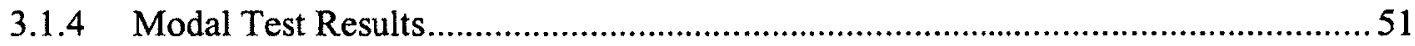

3.2 Identification of Factors that Affect Modal Parameters ............................................53

3.2.1 Variation in Aircrew on Modal Parameters .......................................................53

3.2.2 Variation in Vibration Input Level on Modal Frequencies ..................................56

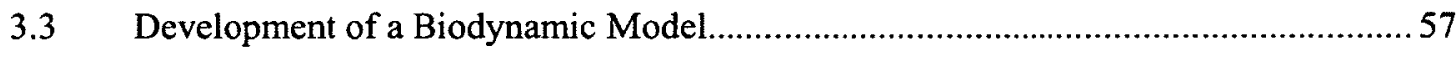

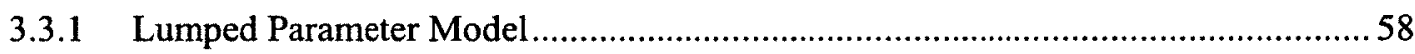

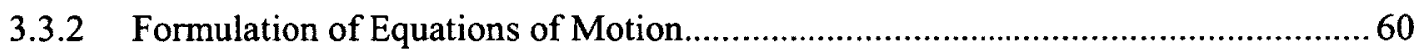

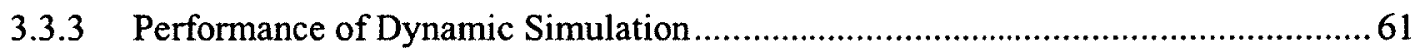

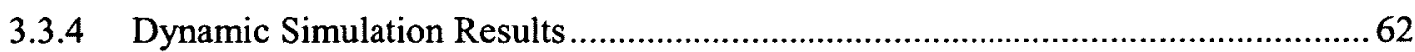

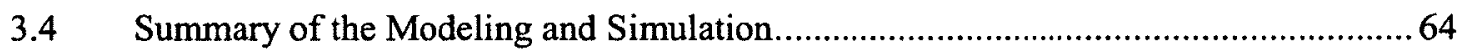

Chapter 4 Adaptive Seat Mount Development and Verification...................... 65

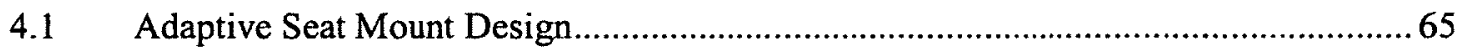

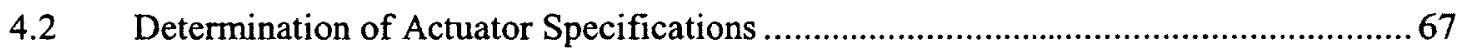

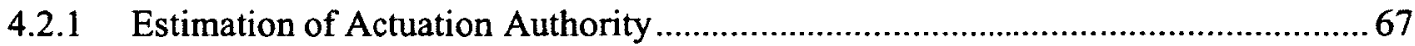

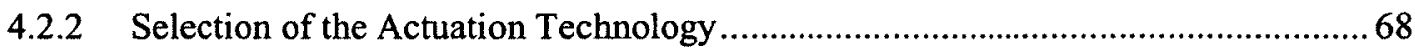

4.3 System Identification of Integrated Seat Hardware ................................................. 72 


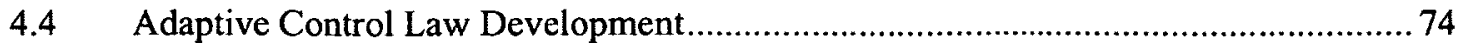

4.4.1 Adaptive Notch Concept to Control Harmonics ..................................................... 75

4.4.2 Control Path Identification Method.............................................................................. 79

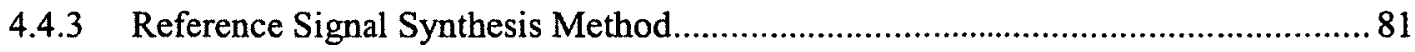

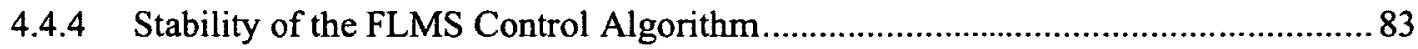

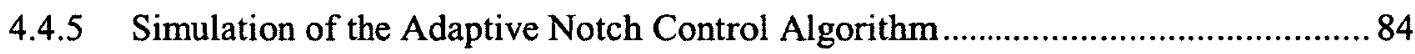

4.5 Feedback Algorithm for Resonance Vibration Control ............................................. 86

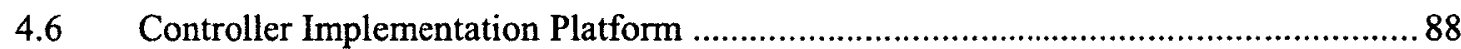

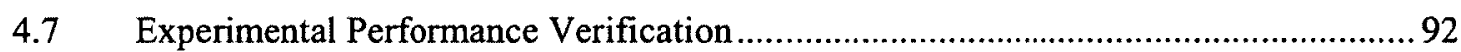

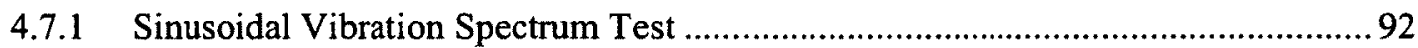

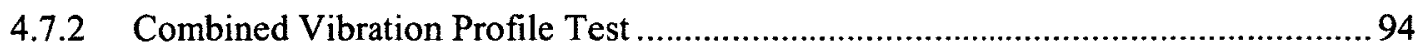

4.7.3 Discussion of the Adaptive Mount Performance …................................................ 95

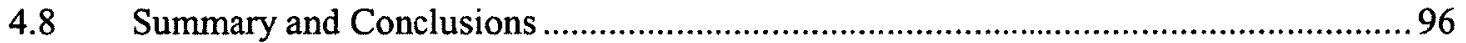

Chapter 5 Investigation of Energy Absorbing Seat Cushion Materials .......... 98

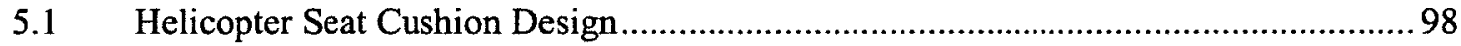

5.1.1 Applicable Cushion Design Requirements .......................................................99

5.1.2 Proposed Cushion Modification Approach .......................................................... 99

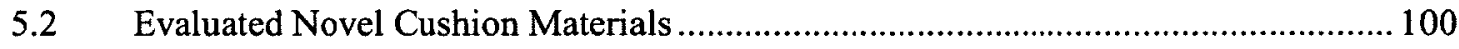

5.2.1 Hybrid Air Cushioning System - Mitigator....................................................... 101

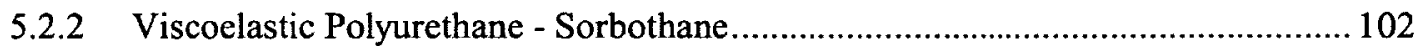

5.2.3 Material Characterization using Shaker Tests................................................... 103

5.3 Mechanical Shaker Tests to Extract Material Properties.......................................... 109

5.4 Flight Tests to Verify Vibration Suppression Performance .................................... 112

5.4.1 Evaluation of Sorbothane Material ….............................................................. 115

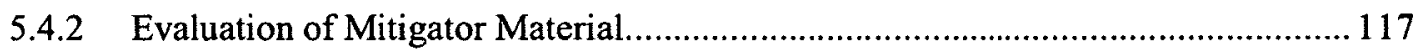

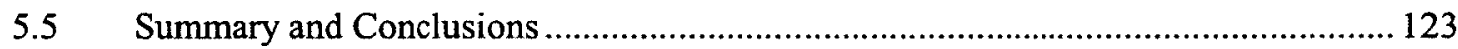

Chapter 6 Conclusions and Recommendations........................................... 125

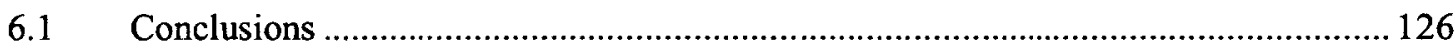

6.1.1 Confirmed the Viability of the Adaptive Seat Mount Methodology for the Helicopter Aircrew Vibration Suppression ....................................................................... 126

6.1.2 Implemented a real-time controller using a feed-forward algorithm and a feedback control law simultaneously. 127 
6.1.3 Verified the High Levels of Vibration Exposure in Helicopter Aircrew in Flight. 127

6.1.4 Demonstrated the Suitability of Novel Energy Absorbing Cushion Material for

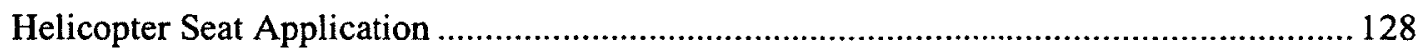

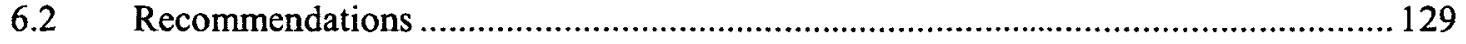

6.2.1 Refinement of the Adaptive Seat Mount Design ............................................... 129

6.2.2 Enhancement of the Biodynamic Seat Model ..................................................... 131

6.2.3 Helicopter Integration of the Actuation System................................................. 131

6.2.4 Expanded Flight Test Program to Survey Cabin Environment ............................ 132

6.2.5 Airworthiness Certification of the Novel Cushion Material ................................. 133

Appendix A Bell-412 Aircrew Vibration Spectra from Flight Tests........................ 135

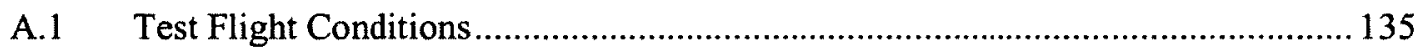

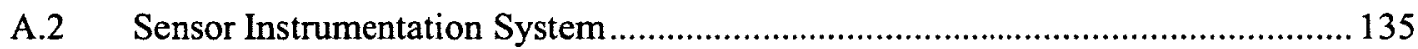

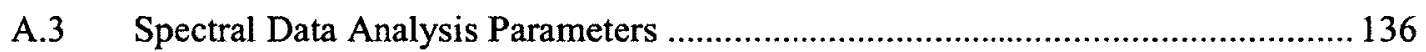

A.4 Measurement Locations and Directions ........................................................... 136

Appendix B State Space Formulation of the Biodynamic Model ............................ 153

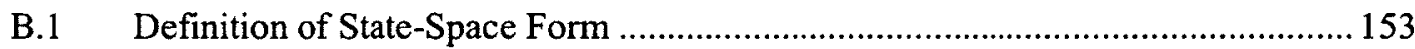

B.2 Derived State-Space Matrices …................................................................... 153

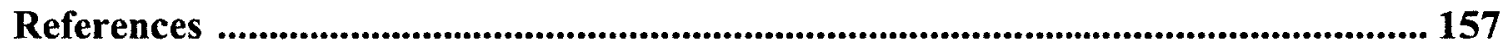




\section{List of Tables}

Table 2.1: Information of accelerometers used in the flight test ................................ 23

Table 2.2: Measured g-rms vibration levels for the pilot locations .............................. 26

Table 2.3: Measured g-rms vibration levels for the co-pilot locations ........................... 26

Table 2.4: rms acceleration ranges for level of comfort [92] ................................... 30

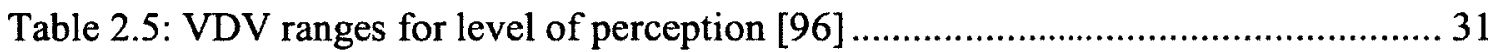

Table 2.6: Measured vibration of the pilot evaluated based ISO 2631 standard ............. 33

Table 2.7: Measured vibration of the co-pilot evaluated based ISO 2631 standard......... 33

Table 3.1: Variation in vibration resonance characteristics for each mannequin ............ 56

Table 3.2: Variation in vibration resonance characteristics with input levels ................ 57

Table 3.3: Mass, stiffness and damping parameters used in the model .........................59

Table 4.1: Actuation requirement based on flight conditions ................................ 68

Table 4.2: Bulk piezoelectric properties [120] ................................................ 69

Table 5.1: Material properties of Mitigator cushions .............................................. 102

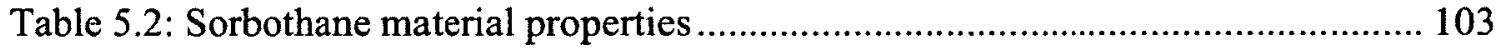

Table 5.3: Extracted material properties of cushion material ................................... 107

Table 5.4: Percentage of reduction for each cushion combination based on g-rms ....... 109

Table 5.5: Flight test matrix to evaluate all selected cushion configuration ................. 114

Table 5.6: Measured g-rms vibration levels for Sorbothane material .......................... 119

Table 5.7: Measured g-rms vibration levels for Mitigator material............................ 120

Table A.1: Vibration spectra with corresponding flight conditions for each test run..... 136 


\section{List of Figures}

Figure 1.1: Sources of helicopter rotor vibration ................................................... 4

Figure 1.2: Schematic of a Higher Harmonic Control (HHC) system .......................... 6

Figure 1.3: Schematic of Individual Blade Control (IBC) systems............................. 7

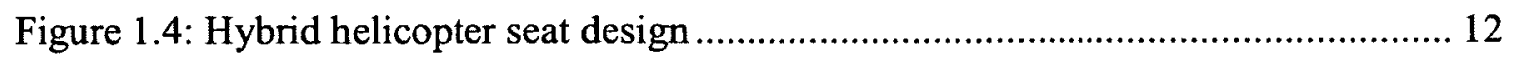

Figure 2.1: Principal directions on the NRC Bell-412 helicopter................................ 18

Figure 2.2: Airspeed and Altitude vs. Time for the Test Flight................................... 20

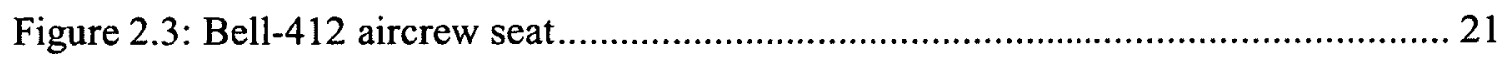

Figure 2.4: Accelerometer locations on the pilot and co-pilot..................................... 22

Figure 2.5: Instrumentation setup in the NRC Bell-412 helicopter ........................... 24

Figure 2.6: ISO 2631 Recommended Acceleration Measurements [92] ....................... 29

Figure 2.7: Pilot helmet vibration in the X-direction................................................... 35

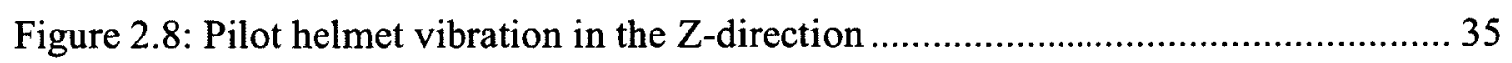

Figure 2.9: Transmission path spectra for the pilot in the Z-Direction for $120 \mathrm{kts}$........ 37

Figure 2.10: Transmission path spectra for the co-pilot in the Z-Direction for $120 \mathrm{kts} . .37$

Figure 2.11: Vibration transmissibility for the helmet location in the Z-Direction.......... 39

Figure 3.1: Seat modal test setup on the shaker with the mannequin ........................... 46

Figure 3.2: Geometry model for the experimental modal test .................................. 47

Figure 3.3: FRFs calculated from the shaker modal tests .................................... 49

Figure 3.4: Stability diagram generated from the modal test..................................... 50

Figure 3.5: Seat modes without mannequin .............................................................. 52

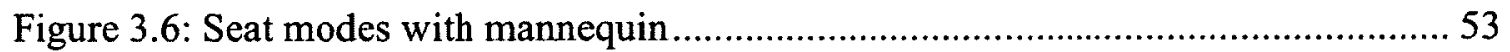

Figure 3.7: Hybrid III mannequins installed on the shaker .......................................55

Figure 3.8: FRFs measured from mannequin head accelerometer in Z-direction ........... 55

Figure 3.9: FRFs measured on the cushion interface in Z-direction............................ 57

Figure 3.10: Lumped parameter model of the seated aircrew .................................... 59

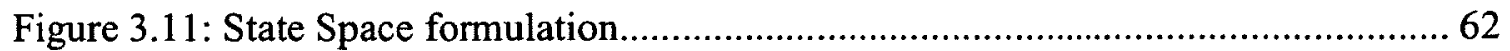

Figure 3.12: Helmet FRF comparison for simulation and flight test ..........................63

Figure 3.13: Time domain response comparison for simulation and flight test ..............63 
Figure 4.1: Mechanical design of the adaptive helicopter seat mount...........................66

Figure 4.2: (a) Interior multilayered stack (b) Selected piezoelectric actuators .............. 70

Figure 4.3: Adaptive seat mount integrated on full-scale Bell-412 seat ....................... 71

Figure 4.4: Modes of the Bell-412 seat integrated with active struts ......................... 73

Figure 4.5: Transfer functions from piezoelectric actuator and mechanical shaker ........ 74

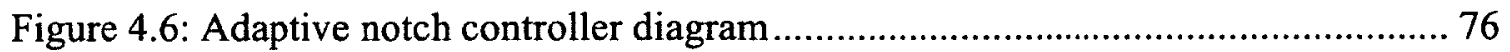

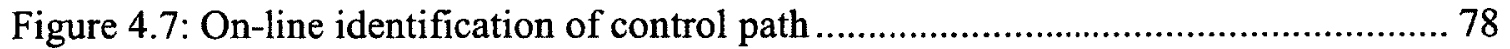

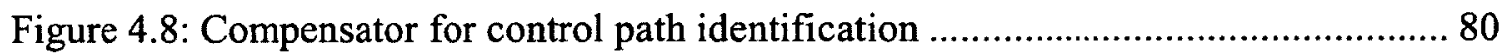

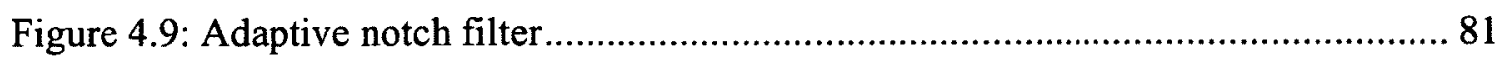

Figure 4.10: Simulation of aircrew helmet vibration in translational flight condition ..... 85

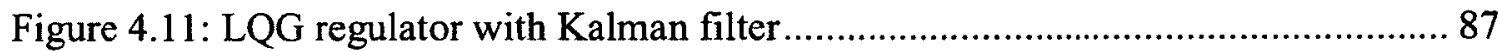

Figure 4.12: Simultaneous implementation of FLMS and LQG algorithms ................. 88

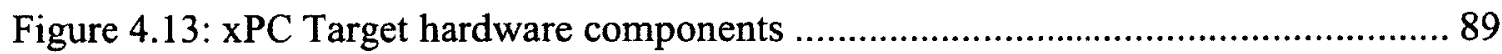

Figure 4.14: Experimental setup with the block diagram of instrumentation................ 91

Figure 4.15: Vibration control results under sinusoidal harmonic profile .................... 93

Figure 4.16: Vibration control results under combined vibration profile ....................... 95

Figure 5.1: Mitigator cushioning structure with interconnected vents ....................... 101

Figure 5.2: Shaker test setup to extract cushion material properties .......................... 104

Figure 5.3: Transfer Function of the original cushion $\left(\xi=0.11\right.$ and $\left.f_{d}=9.00 \mathrm{~Hz}\right) \ldots \ldots . .106$

Figure 5.4: Experimental FRF and dynamic model of combined cushion ................... 108

Figure 5.4: Comparison Mitigator materials using FRF in the $Z$ direction ................. 110

Figure 5.5: Comparison Mitigator materials using FRF in the X direction................. 110

Figure 5.6: Comparison Sorbothane materials using FRF in the $Z$ direction ................ 111

Figure 5.7: Comparison Sorbothane materials using FRF in the $\mathrm{X}$ direction................ 112

Figure 5.8: Cushions materials evaluated using flight test ..................................... 113

Figure 5.9: Aircrew helmet vibration level for selected cushions at 120 kts condition.. 114

Figure 5.10: Spectra of Pilot cushion in the $Z$ direction for Sorbothane material ......... 116

Figure 5.11: Spectra of Co-Pilot helmet in the $Z$ direction for Sorbothane material..... 116

Figure 5.12: Spectra of Pilot cushion in the $Z$ direction for Mitigator material ............ 121

Figure 5.13: Spectra of Co-Pilot cushion in the $Z$ direction for Mitigator material ...... 121 
Figure 5.14: Spectra of Co-Pilot cushion in the X-direction for Mitigator material ...... 122

Figure 5.15: Aircrew helmet vibration with Mitigator urethane for all flight conditions 122

Figure 6.1: Alternate mechanical design of the adaptive seat mount ......................... 130

Figure A.1: Vibration Spectra from Run No. 1 for flight condition ground run (1)...... 137

Figure A.2: Vibration Spectra from Run No. 2 for flight condition $25 \mathrm{ft}$ hover (1)...... 138

Figure A.3: Vibration Spectra from Run No. 3 for flight condition trans. lift (1)......... 139

Figure A.4: Vibration Spectra from Run No. 5 for flight condition $60 \mathrm{kts}$ climb (1).... 140

Figure A.5: Vibration Spectra from Run No. 6 for flight condition $60 \mathrm{kts}$ level (1) ..... 141

Figure A.6: Vibration Spectra from Run No. 7 for flight condition 100 kts level (1) ... 142

Figure A.7: Vibration Spectra from Run No. 8 for flight condition $120 \mathrm{kts}$ level (1) .... 143

Figure A.8: Vibration Spectra from Run No. 9 for flight condition 140 kts descent (1) 144

Figure A.9: Vibration Spectra from Run No. 11 for flight condition 60 kts climb (2)... 145

Figure A.10: Vibration Spectra from Run No. 12 for flight condition 120 kts level (2) 146

Figure A.11: Vibration Spectra from Run No. 13 for flight condition $60 \mathrm{kts}$ level (2).. 147

Figure A.12: Vibration spectra from Run No. 14 for flight condition $100 \mathrm{kts}$ level (2). 148

Figure A.13: Vibration spectra from Run No. 15 for flight condition $140 \mathrm{kts}$ level (1). 149

Figure A.14: Vibration spectra from Run No. 17 for flight condition trans. lift (2)...... 150

Figure A.15: Vibration spectra from Run No. 18 for flight condition $25 \mathrm{ft}$ hover (2) ... 151

Figure A.16: Vibration spectra from Run No. 19 for flight condition ground run (2) ... 152 


\section{List of Abbreviations}

\begin{tabular}{ll} 
ACF & Actively Controlled Flap \\
ACSR & Active Control of Structural Response \\
ACF & Actively Controlled Flap \\
AFC & Active Fiber Composites \\
APL & Active Pitch Link \\
AR & Autoregressive \\
ATR & Active Twist Rotor \\
BVI & Blade-Vortex-Interaction \\
DOF & Degree-of-Freedom \\
ER & Electrorheological \\
FE & Finite Element \\
FFT & Fast Fourier Transform \\
FIR & Finite Impulse Response \\
FLMS & Filtered-x Least Mean Square \\
FRF & Frequency Response Function \\
HACS & Hybrid Air Cushioning System \\
HHC & Higher Harmonic Control \\
HUD & Head-Up Display \\
IBC & Individual Blade Control \\
ISO & International Standard Organization \\
LMS & Least Mean Square \\
LP & Lumped-Parameter \\
LQG & Linear Quadratic Gaussian \\
LSCF & Least-Squares Complex Frequency-Domain \\
LTI & Linear Time Invariant \\
MAC & Modal Assurance Criteria \\
MB & Multi-Body \\
ML & Military \\
\hline
\end{tabular}




$\begin{array}{ll}\text { MFC } & \text { Macro-Fiber Composites } \\ \text { MMIF } & \text { Multivariant Mode Indicator Function } \\ \text { MR } & \text { Magnetorheological } \\ \text { MTVV } & \text { Maximum Transient Vibration Value } \\ \text { NRC } & \text { National Research Council Canada } \\ \text { NVG } & \text { Night Vision Goggle } \\ \text { PZT } & \text { Lead Zirconate Titanate } \\ \text { rev } & \text { Revolutions } \\ \text { rms } & \text { root mean square } \\ \text { SDOF } & \text { Single Degree-of-Freedom } \\ \text { SISO } & \text { Single-Input-Single-Output } \\ \text { VDV } & \text { Vibration Dose Value }\end{array}$




\section{List of Symbols}

\begin{tabular}{|c|c|}
\hline$/$ rev & Non-dimensional frequency \\
\hline$\tau$ & Integration time \\
\hline$\gamma$ & Transmissibility ratio \\
\hline$\mu$ & Convergence coefficient \\
\hline$\lambda_{\mathrm{k}}$ & Eigenvalues of the mode $k$ \\
\hline$\omega_{\mathrm{i}}$ & Harmonic disturbance frequency \\
\hline$\hat{\omega}_{\mathrm{i}}$ & Estimate of the harmonic disturbance frequency \\
\hline$\mu_{1}$ & Convergence coefficient of the compensator \\
\hline a & Acceleration \\
\hline$a_{i}$ & Acceleration for the $\mathrm{i}^{\mathrm{th}}$ frequency spectrum line \\
\hline$a_{i}$ & Coefficients of the autoregressive model \\
\hline$a_{w}$ & Frequency weighted acceleration \\
\hline $\mathrm{A}, \mathrm{B}, \mathrm{C}, \mathrm{D}$ & State Space matrices of the system \\
\hline$A_{f b}, B_{f b}, C_{f b}, D_{f b}$ & State Space matrices of the plant of the feed-back controller \\
\hline c & amplitude of the synthesized reference signal \\
\hline$c_{13}, k_{13}$ & Damping and stiffness between lower and upper torso \\
\hline $\mathbf{C}$ & Damping matrix \\
\hline $\mathrm{d}(\mathrm{k})$ & External disturbance signal \\
\hline $\mathrm{e}(\mathrm{k})$ & Error signal \\
\hline $\mathrm{ee}(\mathrm{k})$ & Error signal of the compensator \\
\hline $\mathrm{E}(\mathrm{z})$ & Error signal in discrete domain \\
\hline$F_{j}(\omega)$ & Fourier transform of a force $f_{j}(t)$ acting in DOF $j$ \\
\hline$f_{j}(t)$ & External force acting in the DOF $j$ \\
\hline$f_{k}$ & Natural frequency of the mode $k$ \\
\hline g-rms & Root mean square acceleration measured in $\mathbf{g}$ \\
\hline $\mathrm{G}(\mathrm{z})$ & Compensator in discrete domain \\
\hline
\end{tabular}




\begin{tabular}{|c|c|}
\hline$G_{x x}$ & Auto-power spectrum \\
\hline$G_{x y}$ & Cross-power spectrum \\
\hline$h(k)$ & Weights of the FIR filter for the control path transfer functionn \\
\hline $\mathbf{H}$ & Transfer function matrix \\
\hline $\mathrm{H}(\mathrm{z})$ & Transfer function of the control path in discrete domain \\
\hline$\hat{\mathrm{H}}(\mathrm{z})$ & Estimated transfer function of the control path in discrete domain \\
\hline$H_{i j}(\omega)$ & Transfer function of the response in DOF $i$ due to force in $\mathrm{DOF} j$ \\
\hline$k_{x}, k_{y}, k_{z}$ & Multiplication factors based on ISO 2631 standard \\
\hline$K$ & Scalar gain of the autoregressive model \\
\hline$K_{f b}$ & Optimal state-feedback gain vector of the feed-back controller \\
\hline $\mathrm{m}$ & Mass \\
\hline $\mathbf{M}$ & Mass matrix \\
\hline $\mathrm{mp}_{1}, \mathrm{cp}_{1}, \mathrm{kp}_{1}$ & Mass, damping and stiffness of the lower torso \\
\hline$m p_{2}, c p_{2}, k p_{2}$ & Mass, damping and stiffness of viscera \\
\hline $\mathrm{mp}_{3}, \mathrm{cp}_{3}, \mathrm{kp}_{3}$ & Mass, damping and stiffness of the upper torso \\
\hline $\mathrm{mp}_{4}, \mathrm{cp}_{4}, \mathrm{kp}_{4}$ & Mass, damping and stiffness of the head and neck \\
\hline $\mathrm{m}_{\mathrm{C}}, \mathrm{c}_{\mathrm{C}}, \mathrm{k}_{\mathrm{C}}$ & Mass, damping and stiffness of the seat cushion \\
\hline $\mathrm{m}_{\mathrm{F}}, \mathrm{c}_{\mathrm{F}}, \mathrm{k}_{\mathrm{F}}$ & Mass, damping and stiffness of the seat frame \\
\hline $\mathrm{n}$ & Number of samples \\
\hline $\mathrm{N}$ & Number of blades \\
\hline o & Number of frequencies to be included in the reference \\
\hline$p_{k}$ & Pole of the mode $k$ \\
\hline $\mathbf{P}$ & Arbitrary Integer \\
\hline$q$ & Model order of the autoregressive model \\
\hline$q_{k}$ & Generalized coordinates of the mode $k$ \\
\hline$Q_{f b}, N_{f}, R_{f b}$ & Matrices for state weighting, noise weighting, control weighting \\
\hline$r_{k, i j}$ & Residue of mode $k$ at DOF $i$ with reference to DOF $j$ \\
\hline $\mathrm{R}$ & Number of DOFs \\
\hline
\end{tabular}




\begin{tabular}{|c|c|}
\hline $\mathbf{S}$ & Stiffness matrix \\
\hline$S_{F X}(\omega)$ & Cross-spectrum of $x_{i}(t)$ and $f_{j}(t)$ \\
\hline$S_{F F}(\omega)$ & Auto- spectrum of $f_{j}(t)$ \\
\hline$t$ & Integration variable \\
\hline$t_{0}$ & Instantaneous time \\
\hline$T$ & Length of operating time \\
\hline $\mathbf{u}$ & Input vector with position and velocity of the helicopter floor \\
\hline $\mathrm{u}(\mathrm{k})$ & Control input signal \\
\hline$u_{f b}$ & Control signal of the feed-back controller \\
\hline$v_{f b}$ & Sensor noise of the feed-back controller \\
\hline$v(k)$ & White noise \\
\hline $\mathbf{v}_{k}$ & Eigenvector of the mode $k$ \\
\hline $\mathrm{w}(\mathrm{k})$ & Iterative weight coefficients of the FIR filter for the controller \\
\hline$w_{f b}$ & Process noise of the feed-back controller \\
\hline$W_{i}$ & Weighting factor for acceleration based on ISO 2631 standard \\
\hline $\mathrm{W}(\mathrm{z})$ & Adaptive weights of the controller \\
\hline$X(f)$ & FFT of a signal \\
\hline$X^{*}(f)$ & Complex conjugate of $X(f)$ \\
\hline$X_{i}(\omega)$ & Fourier transform of the response $x_{i}(t)$ in DOF $i$ \\
\hline $\bar{x}$ & Mean value of the single \\
\hline$x_{i}(t)$ & Deflection in the DOF $i$ \\
\hline $\mathrm{x}(\mathrm{k})$ & Synthesized reference signal \\
\hline$\hat{\mathbf{x}}(\mathbf{k})$ & Filtered reference signal \\
\hline$x_{f b}$ & State vector of the feed-back controller \\
\hline$\hat{\boldsymbol{x}}_{f b}$ & Estimated state vector of the feed-back controller \\
\hline $\mathbf{y}$ & Output vector with positions of the aircrew helmet \\
\hline$y(k)$ & Output due to controller input \\
\hline$y_{f b}$ & Measurement vector of the feed-back controller \\
\hline $\mathrm{Y}(\mathrm{z})$ & Output signal in discrete domain \\
\hline
\end{tabular}




\section{Chapter 1}

\section{Helicopter Aircrew Vibration and Control Approaches}

The helicopter has become a versatile mode of aerial transportation primarily due to its unique capability to take-off and land vertically as well as its ability to hover. These characteristics of helicopters enable many unique tasks such as rescue operations in civilian use and reconnaissance in military applications. The use of helicopters has increased in the last few decades, especially in civilian applications, and this trend is expected to continue in the future.

\subsection{Motivation}

Despite these unique abilities, helicopter flights are generally unpleasant for aircrew as well as passengers due to their exposure to high vibration levels in the cabin. The cabin vibration is primarily excited by the rotor blade aerodynamic and inertial loads at $\mathrm{N} / \mathrm{rev}$ harmonic frequencies. The cabin floor vibration is transmitted through the seat and exposes the aircrew to whole body vibrations. In the short-term, the mechanical vibration transmitted to the human body increases fatigue, degrades comfort, interferes with effective performance, and diminishes operational safety [1]. In addition, the continuous exposure to repetitive vibrations transferred through the helicopter seats have been known to cause damaging effects on the spine and neck of the aircrew leading to longterm occupational health issues [2]. Recent reports of adverse health effects, which 
include pilot neck and spine strain injuries due to whole body vibration exposure, have become a major concern among the rotary aircraft aircrew communities $[1,3]$. These vibration induced health issues become more severe when additional instruments, such as the Head-Up Display (HUD) and Night Vision Goggle (NVG) systems with associated counter weight, are integrated onto helicopter pilot helmets in military operations. The inclusion of additional devices such as HUDs and NVGs onto the aircrew helmet under a high vibratory environment has been suggested as a cause in recent reports of back pain and neck strain $[4,5]$. Extended mission durations and back-to-back missions are other factors that contribute to increased health issues in military helicopter aircrew. For example, approximately $80 \%$ of Canadian helicopter pilots have reported experiencing neck and back pain related to flying the $\mathrm{CH}-146$ Griffon helicopter, and $70 \%$ of pilots reported experiencing neck pain during actual flights [3]. Similar flying related pain has also been reported in Swedish (57\%) [6] and Australian (29\%) [7] militaries. Unfortunately, these health problems reported by the Canadian $\mathrm{CH}-146$ helicopter crew lead to temporary loss of flight status that affect aircrew availability for missions and in some extreme cases lead to permanent disability claims that require costly training of new aircrew while causing substantial expense to the health care system [8]. Therefore, the reduction of vibration levels experienced by the helicopter aircrew is essential to enhance the ride quality of the vehicle and to mitigate health problems of the rotary wing community.

An immediate and viable approach to reduce vibration transmitted to helicopters aircrew and passengers is through advanced seat designs. In general, helicopter seat structures are required to meet both crashworthy criteria and vibration isolation performance in accordance to MIL standards $[9,10]$. For current helicopter seat designs, the primary vibration isolation is provided passively through the use of seat cushions. Unfortunately, the impedance properties of the seat cushions are difficult to optimize without compromising pilot handling quality as well as seat comfort. Varied aircrew weight and continuously changing flight conditions also impose practical limitations on the selection of helicopter seat cushion properties and characteristics. Therefore, most seat cushions are designed to be relatively firm in order to avoid excessive displacement 
during flight manoeuvres and therefore the vibration isolation performance may be relatively poor, especially in the low frequency regime.

A novel hybrid helicopter seat that includes an adaptive seat mount approach and novel energy absorbing cushion materials are investigated in this study to reduce the vibration effects of the aircrew. The adaptive seat mount design incorporate novel piezoelectric actuators controlled using a real-time control algorithm to counteract transmission of seat vibration to the aircrew. In addition, this investigation also evaluated the use of novel energy absorbing cushion material as a low cost, near term, solution to improve aircrew vibration exposure. Although seat structures in helicopters are considered to be an essential part of the crash safety system, these approaches are considered to provide viable solutions to mitigate aircrew vibration because the certification requirements for seats are significantly less stringent than flight critical components such as helicopter blades. Therefore, these hybrid vibration suppression approaches based on novel active and passive methodologies for the helicopter seat application were developed in this study and their effectiveness was evaluated using fullscale system implementation under representative vibratory conditions.

\subsection{Background in Helicopter Vibration Control}

High levels of structural vibration due to unsteady aerodynamics and inertial loads in the rotor blade environment are a notable and undesirable characteristic of helicopters [11]. The most important sources that contribute to the vibration in a helicopter airframe are the main rotor hub reactions that corresponds to the harmonics of the blade passage frequency induced by the inertial loads as well as aerodynamic loads due to phenomena such as blade-vortex-interaction (BVI) and dynamics blade stall, et cetera as illustrated in Figure 1.1. The rotor vibration energy transferred throughout the helicopter structure may not only lead to fatigue damage of expensive components and higher maintenance costs but also may create a severe environment for passengers and aircrew. In order to reduce vibration in helicopters, the helicopter industry as well as the rotor dynamic 
research community has worked diligently in order to overcome undesired vibrations in helicopters using both passive as well as active vibration control approaches.

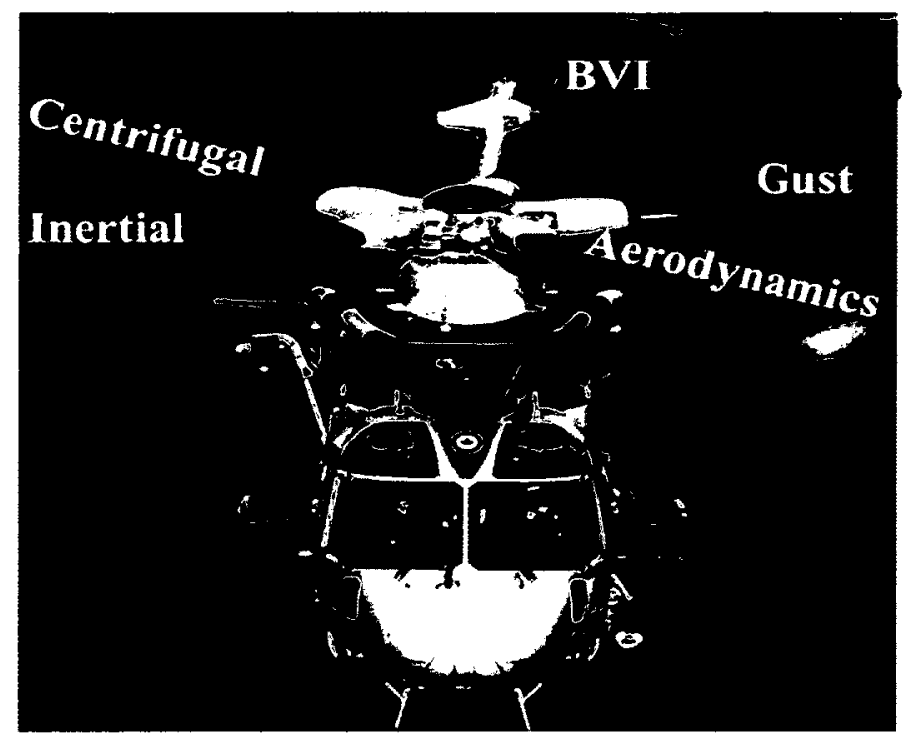

Figure 1.1: Sources of helicopter rotor vibration

\subsubsection{Passive Vibration Control for Helicopters}

A variety of passive vibration absorbers and isolators have been applied in helicopters to reduce structural vibration and noise. Passive control devices provide a moderate reduction in vibration with no risk of instability and lower complexity, compared to active devices [12]. Passive devices are used in many operational helicopters by tuning them to filter out specific disturbance frequencies. Most passive approaches are used in the helicopter cabin such as the seat cushions and trim panels, but a few studies have investigated the use of passive devices on the rotor blades themselves [13]. For example, pendulum absorbers can be mounted at the root of the blade to create a node at a given frequency. Significant reductions in the amplitude of specific vibratory frequencies have been achieved with such systems [14]. Isolation systems can also be implemented between the fuselage and the drive unit and the rotor [15].

In general, passive approaches designed for high vibration reduction performance results in undesirable weight penalties for helicopter application. One of the main 
disadvantages of using passive dampers to reduce vibration in helicopters is that passive devices are generally tuned to a specific frequency. Due to the fact that helicopter vibration consists of wider spectrum of frequencies, including many harmonics of the primary rotor vibration, passive vibration isolation or passive damping only have limited success [16]. Therefore more complex active vibration control approaches are currently being investigated and implemented as a means of reducing vibration in helicopter flight. The most commonly used active vibration reduction strategies for helicopters include active rotor control to achieve global reduction and Active Control of Structural Response (ACSR) for local vibration reduction targeted at critical areas or specific components.

\subsubsection{Active Rotor Control for Helicopters}

Active rotor control concepts have been investigated in the past as means of reducing vibration in helicopters but few have been implemented. Due to the stochastic nature of the rotor dynamics in flight and broadband of rotor harmonics, the active feedback control using actuators would lead to greater performance benefits in comparison to passive approaches [17]. Although active control systems can be very effective in reducing helicopter vibration, many of the active vibration reduction techniques have never been applied to production helicopters for several reasons, with the primary reason being ensuring the safety of the vehicle due to modifications required for flight critical components. Active systems that utilize the primary flight control system of the helicopter such as the pitch links or swash plate for vibration reduction purposes are required to achieve stringent airworthiness certification criteria. In addition, active rotor concepts require electrical or hydraulic slip rings susceptible to failure and require significant amounts of power to drive the actuators located on the rotor. Additional drawbacks include the requirement of high force actuation, a significant weight penalty and stability issues pertaining to the rotor system. In general, active rotor control approaches have been investigated based on two overall concepts, namely, Higher Harmonic Control (HHC) and Individual Blade Control (IBC). 


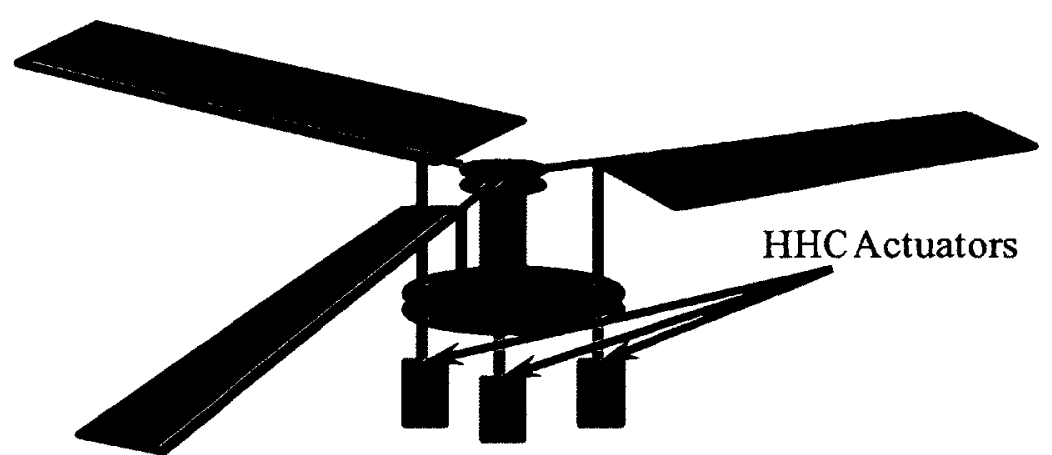

Figure 1.2: Schematic of a Higher Harmonic Control (HHC) system

In HHC, the whole rotor system comprising of all the blades is treated as the source of vibration and the feedback control inputs are applied to the whole system through the swashplate as shown in Figure 1.2. One of the earliest studies in HHC was performed by Kaman with Controllable Twist Rotor where a training edge servo-flap was used for controlling the dynamic behavior of the rotor system [18]. Full-scale wind tunnel tests of this rotor by Lemnios and McCloud showed that a mechanically controlled servo-flap in combination with a torsionally soft rotor blade could be used to redistribute the lift on the rotor disk through HHC [19]. HHC using swash plate control of blade pitch was applied by Shaw in a comprehensive wind tunnel study of a 1/6 Mach-scale $\mathrm{CH}-47 \mathrm{D}$ rotor [20]. Nguyen and Chopra performed an analytical study of the same rotor and confirmed these results [21]. A full-scale flight demonstration of the HHC system was carried out on a modified Sikorsky S-76A helicopter [22]. It is important to note that the HHC concept results in a rigid-body pitch actuation of the whole rotor system through the swash plate to reduce vibration in helicopters.

In contrast to $\mathrm{HHC}$, the IBC concept treats each rotor blade as a source of vibration and independent feedback control input is applied to each of the rotor blades [23]. There are many advantages of placing actuators closer to the source of vibration at the blade. These advantages include improved vibration control, higher noise reduction, more degrees of freedom, et cetera. Furthermore, the blade mounted actuators dedicated for vibration control would not be considered a flight critical system and thus the failure of actuators would not lead to safety of flight issues. The significant advantages of mounting the actuators directly on the blades has lead to in-depth studies of various 
actuator types that could be used to implement the IBC concept. For example, IBC approaches have been investigated to actively control the blade pitch links (APL), trailing edge active control flap (ACF), or active twist rotor (ATR) for blade twist control for vibration reduction as shown in Figure 1.3. These actuators must provide sufficient authority to apply the desired control while meeting inertial and geometric constraints of the rotor blade.

IBC concepts using the APL approach were implemented using electromagnetic or hydraulic actuators connecting blade and swash plate, replacing conventional pitch links [24]. A full-scale IBC system using blade pitch control was demonstrated in the NASA Ames $40-\mathrm{ft}$ by $80-\mathrm{ft}$ Wind Tunnel by Jacklin et al. using a 4-bladed BO-105 hingeless rotor [25]. In addition, several flight tests of the APL system for vibration reduction have been conducted successfully in the past [26,27]. Recently, an APL with semi-active impedance control system to control the blade torsional stiffness has also been investigated by Nitzsche et al. [28,29]. The mechanical impedance properties of the pitch link are actively controlled through a device known as the Smart Spring [30,31]. The performance of APL has also been demonstrated under centrifugal loads to reduce vibration in whirl tower rotor tests [32].

The most popular IBC approach is the trailing edge ACF that has been pursued by several research teams $[33,34,35,36]$. This approach uses a small flap on each blade to generate controlled aerodynamic loads for vibration reduction. The location and size of the flaps can be chosen optimally to increase the efficiency of the flap system while the failure of the ACF does not compromise the primary flight control system of the helicopter. Although many types of actuation mechanisms have been employed in the

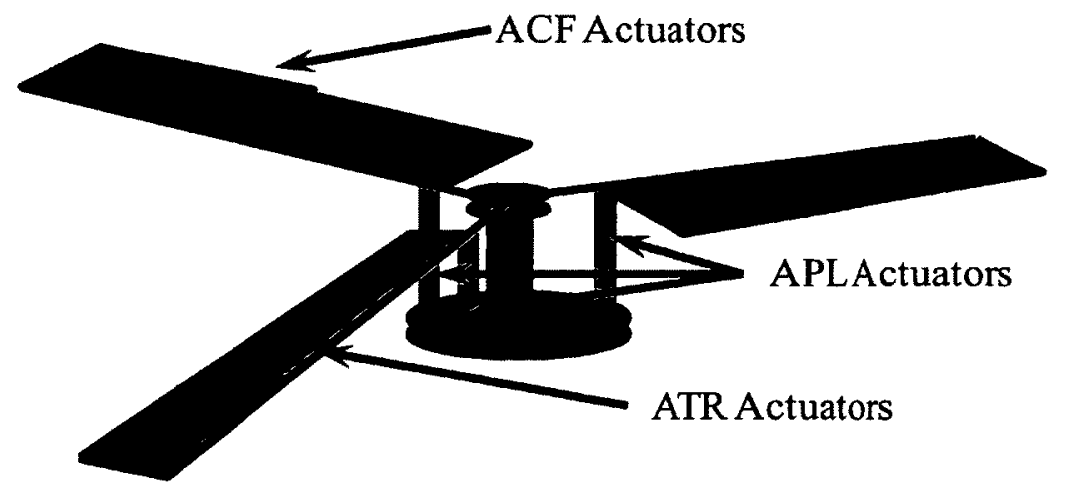

Figure 1.3: Schematic of Individual Blade Control (IBC) systems 
past, the use of novel smart materials actuators such as piezoelectric actuators $[37,38,39]$ and magnetostrictive actuators $[40,41,42]$ have enhanced the practicality of the ACF approach for helicopter application. The vibration suppression performance of the ACF for helicopter application has been demonstrated in wind tunnel tests $[43,44]$ and a blade integrated with an ACF was flown by Eurocopter recently [45]. Although the ACF approach offers excellent promise for vibration reduction, many practical issues such as the transfer of power to the rotating blade is a challenge for the flap system implementation. In addition, the actuator needs to be sufficiently powerful to provide the required control while small enough to be integrated within the blade cavity. Furthermore, complex flap to actuator linkage requires smooth operation under the high centrifugal loading of the blade.

ATR is another IBC approach that actively twists the blade using distributed conformable smart material embedded in the blade to alleviate the unsteady aerodynamic loads to achieve vibration reduction. The ATR concept has been investigated by using conformable smart materials, such as Active Fiber Composites (AFC) $[46,47]$ and Macro-Fiber Composites (MFC) [48] which uses piezoelectric material for actuation. Extensive electromechanical material characterization has been performed to evaluate AFCs and MFCs both as actuators and as structural component of the blade $[49,50]$. One of the advantages is the simplicity of its actuation mechanism compared to actuation of the ACF located at the trailing edge. The ATR concept has merit in that it does not increase the profile drag of the blade as does the discrete ACF. Although the ATR technology produces significant vibration reduction, the power required for vibration reduction is expected to be higher than that for ACF [51]. Wilbur et al. tested a prototype active-twist blade in the hover condition at the NASA Langley Research Center to demonstrate the vibration suppression performance and endurance characteristics of the prototype blade [52]. Shin and Cesnik established the performance of the active twist blade under forward flight conditions by performing open and closed-loop control tests in the wind tunnel [53]. Similar investigations in the ATR concepts using embedded piezoceramic elements have been conducted by a number of research teams $[54,55]$. Although investigations have shown that ATR technology is able to produce significant 
reduction in vibration in helicopters, the challenge common to other IBC approaches, specifically that of the transfer of power to the rotating blades through slip rings remains a major impediment to the practical implementation in production vehicles.

\subsubsection{Active Control of Structural Response (ACSR)}

Recent research efforts in helicopter vibration control not only focused on the global vibration reduction but also on the local vibration reduction that targets specific locations in the structure. Although it is highly desirable to reduce the unsteady aerodynamic loads from the rotor blades in order to achieve a global suppression of helicopter vibration levels, the successful applications on full-scale vehicles are limited due to lack of high efficiency actuators, heavy weight penalties, system complexities and stringent certification requirements for flight critical components. An alternate approach which is known as ACSR, employs one or more active devices to suppress vibration locally. Unlike the HHC and IBC concepts which are intended for global vibration reduction through the active control of the rotor system, the ACSR approach is designed to attenuate the vibration in specific locations such as the cabin or the aircrew seat. ACSR is one of the most successful vibration reduction methods that has been used in helicopters at the present time. ACSR systems include vibration sensors that are placed at key locations to monitor vibration and an efficient controller with a suitable control algorithm to calculate proper actions for actuators to reduce the vibration at a particular location of the helicopter. Actions determined by the controller are fed to appropriate actuators, located optimally to provide the desired actuation forces. Comprehensive investigations of various ACSR approaches have been conducted through analytical studies as well as experimental tests. For example, Chui and Friedmann conducted an analytical investigation of helicopter vibration reduction using the ACSR technique [56]. The results showed substantial reduction in the fuselage vibration using an ACSR system with four force actuators used to mount the rotor assembly into the fuselage [57]. Furthermore, flight tests have also been conducted to demonstrate the effectiveness of the ACSR systems on a Westland 30 helicopter [58] and an UH-60 Black Hawk helicopter [59]. In addition, Welsh et al. modified a Sikorsky S-76B helicopter with 4 servo force 
actuators and 10 accelerometers monitoring airframe vibrations to demonstrate that an ACSR system was effective in reducing airframe vibration under varying rotor speed [60]. Successful integration of ACSR technology has allowed the helicopter industry to incorporate ACSR systems in modern production helicopters such as the Westland EH101 [61] and the Sikorsky S-92 Helibus [62].

Although the ACSR systems provide a viable solution for helicopter vibration reduction, these systems generally require high power for effective performance. In addition to the actuators, sensor and controller weight, the weight penalty is also increased because the actuation locations have to be designed specially to accommodate large forces without compromising structural integrity.

\subsection{Adaptive Helicopter Seat Mounts}

The adaptive helicopter seat mount is a subset of the ACSR specifically designed for seat structures to reduce vibration transmitted to the helicopter aircrew and passengers while avoiding excessive weight penalty and power requirements. Traditionally, helicopter seats are designed primarily to meet crashworthy criteria while vibration isolation is achieved passively through the seat cushions. However, the impedance properties of the conventional passive seat cushion materials are difficult to optimize without compromising pilot handling qualities and seat comfort. Most seat cushions are designed to be relatively firm to avoid excessive displacement during flight manoeuvres. Varying pilot weight and flight configurations also impose compromises in the design of helicopter seat cushions. As a direct approach with near-term implementation horizon to reduce vibration transmitted to helicopter aircrew, many researchers have been motivated to investigate innovative seat mount designs and suspensions systems to improve vibration suppression performance.

\subsubsection{Current State of Adaptive Seat Mount Developments}

In contrast to passive cushions, an adaptive seat mount is able to overcome the variation in vibratory frequencies due to changes in aircrew size and flight configuration through 
embedded adaptive control systems. Moreover, adaptive seat mount systems do not require significant changes to helicopter structures or modifications to the rotor and flight control systems. Therefore, the adaptive seat mount approach offers a viable solution to reduce the harmful effects of vibration on helicopter aircrew health over broad operating conditions. Adaptive seat mount concepts have been investigated using both active approaches that counteract vibration using actuators as well as semi-active strategies that modify structural properties, such as stiffness or damping to achieve vibration reduction.

Several adaptive seat mounts that employ semi-active approaches such as magnetorheological (MR) and electrorheological (ER) dampers have been investigated for heavy industrial vehicles $[63,64]$. Choi et al. investigated seat vibration reduction systems using skyhook and sliding mode control algorithms with both ER and MR dampers for a commercial vehicle $[65,66]$. Wu and Griffin studied several semi-active control algorithms to reduce seat vibration using suspension systems to demonstrate that a compromise can be made between vibration isolation and end-stop impact reduction [67]. Recently, Choi and Wereley analytically evaluated the response of the seated human occupant controlled through a MR damping mechanism integrated on to a rotorcraft seat [68]. Heimenz et al. demonstrated that the semi-active MR damper based suspension system for helicopter seat showed significantly better vibration attenuation performance than passive seat suspensions $[69,70]$. An advantage of semi-active systems is that they require less power when compared to active systems. In addition, they are relatively reliable and fail-safe for practical implementation. However, the vibration suppression performance of semi-active approaches is generally more modest when compared to active concepts.

\subsubsection{Active Control Technology for Adaptive Seat Mounts}

An active system with actuators controlled by a real-time control algorithm applied as an adaptive helicopter seat mount is able to exploit its characteristics to counteract vibration under varying conditions such as aircrew weight, flight condition, et cetera. Compared to vibration suppression performance of semi-active approaches, the active control concepts are generally superior in counteractive forced vibrations such as $\mathrm{N} / \mathrm{rev}$ harmonics 
vibration excitation of helicopter seats. In addition, active control systems are able to operate effectively in a wide range of frequencies in a controlled manner to optimize system performance. In principle, the power required for the seat application is expected to be significantly less compared to cabin ACSR systems due to lower vibration excitation levels and the application is localized only to the seat structure. Moreover, adaptive seat mount systems do not require significant modifications to helicopter critical structural components such as the rotor blades or the flight control systems. It is important to note that studies that apply active control approaches specifically to helicopter seats to reduce vibration experienced by the aircrew could not be found in the literature. Therefore, this novel adaptive seat mount concept based on active control technology offers a viable solution to reduce the harmful vibration effects of helicopter aircrew health over broad operating conditions.

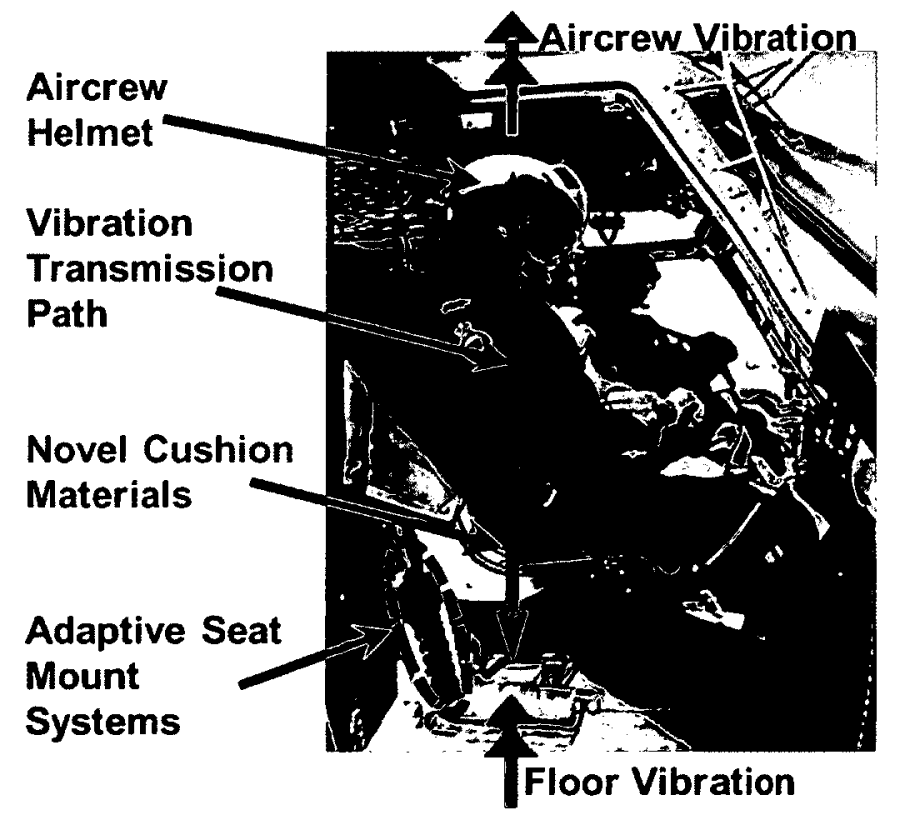

Figure 1.4: Hybrid helicopter seat design

\subsection{Objectives of the Thesis}

In this research study, both active and passive approaches are being investigated for the helicopter seat application to suppress the vibration transmitted to the aircrew as 
illustrated in Figure 1.4. This study is primarily focused on developing a novel adaptive seat mount system based on active vibration control technology to counteract forced vibration frequencies generated by the rotor. In addition, this study also evaluates the use of novel energy absorbing material to dampen the resonance vibration. Therefore, this novel hybrid seat system with active and passive approaches offers a viable solution to reduce the harmful vibration effects on helicopter aircrew health over a wide range of frequencies due to broad operating conditions.

The objective of this thesis is to develop a novel hybrid helicopter seat system that includes passive energy absorbing cushion materials as well as an adaptive seat mount approach based on active control technology to suppress transmission of vibration to the aircrew. The effectiveness of the hybrid vibration suppression approaches were evaluated using full-scale hardware under representative vibratory conditions. In particular, the hybrid seat was designed for retrofit into the Bell-412 helicopter to mitigate the transmission of vibration to the aircrew. The Bell-412 helicopter is the civilian version of the $\mathrm{CH}-146$ aircraft used by the Canadian military that has been reported by the aircrew to cause pain and injuries. These objectives of the thesis are achieved by:

1. Performing vibration measurements of helicopter aircrew as well as the helicopter structure under representative flight conditions to determine the characteristics of the aircrew vibration profile.

2. Performing experimental modal tests to extract the dynamic characteristics of the seated aircrew through shaker tests to develop an efficient biodynamic model for vibration simulation.

3. Developing a validated lumped parameter based biodynamic model of the seated aircrew to efficiently evaluate the effectiveness of various seat design options as well as cushion materials to mitigate vibration transmission to helicopter aircrew.

4. Developing a suitable hardware system using active material actuator technologies to retrofit a full-scale helicopter seat to counteract the vibration transmitted to the aircrew. 
5. Implementing a real-time control algorithm to adaptively control the piezoelectric actuators integrated with the seat structure to suppress the helicopter vibration transmitted through the seat.

6. Performing laboratory tests to demonstrate the effectiveness of the novel adaptive seat mount system implemented on full-scale helicopter seat hardware under representative helicopter vibratory environment.

7. Investigating novel energy absorbing cushion materials to dampen resonance vibration of the seat and performing flight tests to verify the vibration suppression performance of selected cushion materials.

\subsection{Original Contributions}

This research represents the first known investigation of an adaptive mount system based on active control technology on full-scale helicopter seats to suppress aircrew vibration. In addition, unique flight tests were performed to acquire vibration data from aircrew under typical flight conditions and evaluate novel energy absorbing cushion material. As such, there are a number of original contributions generated during this research study in the development of a full-scale hybrid helicopter seat integrated with passive and active approaches to mitigate aircrew vibration as follows:

1. Characterize helicopter aircrew vibration profile using flight tests conducted on a Bell-412 helicopter. Although vibration data on helicopter structures, such as floor and seat, have been published previously, vibration data on aircrew cannot be found in published literature. Therefore, a unique flight test was performed to acquire vibration data from helicopter aircrew under typical flight conditions.

2. Develop a lumped parameter based biodynamic model of the seated aircrew to efficiently evaluate the effectiveness of various seat design options to mitigate vibration transmission to helicopter aircrew through dynamic simulations. The uniqueness of this model was that the cushion and seat frame dynamics were separated to independently evaluate the effect of cushion properties on aircrew vibration. 
3. Develop an adaptive seat mount based on active control approaches using stacked piezoelectric material actuators controlled by a real-time control algorithm to counteract forced vibration transmitted to the aircrew. Although semi-active approaches have been used previously for helicopter seat applications, the use of piezoelectric actuator based active control technology for helicopter seat application could not be found in published literature.

4. Implement FLMS feed-forward control algorithm based on adaptive notch filters to suppress forced vibration frequencies as well as a robust feedback control law to control resonance modes simultaneously on a full-scale helicopter seat structure. The vibration suppression performance of the adaptive seat mount performed using a shaker showed viability of the proposed approach for aircrew vibration reduction under representative helicopter vibratory environment.

5. Investigate novel energy absorbing cushion materials for to dampen resonance vibration through Bell-412 helicopter flight tests. Selected novel cushion materials showed appreciable suppression of the aircrew vibration without significant modifications to the seat system. It is recommended to pursue the retrofit integration of these novel cushions to current helicopter seats as a low cost solution that only requires minimum certification effort.

It is important to note that the progress of this research work has been published during the course of the work in several international conferences and these presentations have been well received by the technical audience $[71,72,73,74,75]$. More importantly, significant outcomes of this study have already been published in a number of prominent peer reviewed journals in the field of aircraft vibration control, which verifies the originality of the research by the broader scientific community $[76,77,78,79]$. In addition, a number of these publications have been cited by over 10 technical papers authored by several prominent researchers across the world $[80,81,82,83,84,85]$. This attests not only to the originality of the findings of this research but also signifies the importance of this type of investigation to identify solutions to mitigate adverse health effects in helicopter aircrew due to exposure to high levels of vibration. 


\subsection{Organization of the Thesis}

Chapter 1 presents the motivation for this research and a brief background on need for helicopter seat vibration control. It included a detailed background regarding the need to reduce vibration exposure on helicopter aircrew and various approaches studied as potential solutions for vibration control in helicopters. The significance of the thesis, objectives and the main contributions are given in the present chapter.

Chapter 2 provides details of the flight test performed to characterize the vibration spectra of the aircrew under representative helicopter flight conditions. Characteristics of the aircrew vibration are required to develop an effective adaptive seat mount to mitigate vibration transmitted through the seat. It describes the flight test methodology, the data acquisition system, the data analysis technique and discusses significance of the measured aircrew vibration data from the flight test.

Chapter 3 discusses comprehensive biodynamic modeling effort that included a variety of techniques to characterize structural dynamics of the seated aircrew. This included base excited modal test using mechanical shaker tests to extract modal parameters of the seat with a strapped mannequin. Extracted parameters are used to develop a detailed lumped parameter model for vibration simulation.

Chapter 4 describes the comprehensive design methodology used to develop an adaptive seat mount system using piezoceramic actuators and a real-time control algorithm. It includes actuator selection, full-scale hardware integration, adaptive controller design, real-time control system implementation and performance verification of the adaptive seat mount using representative helicopter vibration using a mechanical shaker excitation. Chapter 5 provides details of the novel energy absorbing cushion material selection process perused to dampen resonance vibration. It provides the details of the flight test performed to verify the effectiveness of novel cushion materials for helicopter seat application and highlights the necessity to pursue the use of passive cushions as a nearterm solution for aircrew vibration suppression.

Chapter 6 concludes by summarizing the results of this research effort and provides recommendations for future work. 


\section{Chapter 2}

\section{Flight Tests to Characterize Helicopter Aircrew Vibration}

As a pre-requisite to develop an effective adaptive seat mount to mitigate vibration transmitted through the seat, it was necessary to identify the vibration spectra of the aircrew under representative helicopter flight conditions. Although some structural vibration data of the Bell-412 helicopter is available in the published literature, aircrew vibration data is not available. Therefore, a flight test was conducted to acquire the aircrew and structural vibration data using the NRC Bell-412 aircraft under representative flight conditions. This chapter describes the flight test methodology and analyses of the data in both the time and frequency domain. In addition, the aircrew vibration environment was assessed based on commonly used and accepted evaluation standards to rate comfort, health and perception.

\subsection{Helicopter Seat Vibration}

In general, vibration in the helicopter cabin is dominated by the vibration that originates from the main rotor [86]. Therefore, it was expected that the vibration of the seat as well as that experienced by the aircrew will also be dominated by the main rotor vibration. Most of the aerodynamic vibratory loads produced by the rotor system are cancelled at the hub, except for their $\mathrm{PN} /$ rev and $\mathrm{PN} \pm 1$ /rev harmonics, where $\mathrm{P}$ is an arbitrary integer and $\mathrm{N}$ is the number of blades [87]. In addition, the imbalance characteristics of the 
blade generates $1 /$ rev excitation on the hub. Due to the inherent coupling between the rotor system and the airframe, vibratory hub loads are transferred throughout the helicopter structure. For the Bell-412 helicopter, which has a 4 bladed rotor system, the $4 / \mathrm{rev}$ frequency is approximately $21.6 \mathrm{~Hz}$ and the $1 / \mathrm{rev}$ frequency is approximately 5.4 $\mathrm{Hz}$. In order to acquire the relevant vibration data from the aircrew, a flight test methodology was formulated in collaboration with the aircrew, aircraft technicians and airworthiness certification personnel at NRC. The collaborative effort of the multiple stake holders resulted in a detailed flight test plan as discussed below.

\subsection{Flight Test Plan}

A comprehensive flight test plan was developed to acquire aircrew vibration data at representative flight conditions while minimizing the flight test time and reducing the flight certification burden due to the addition of test instrumentation in the aircraft [88]. Reduction of flight test time was important in order to reduce the overall cost of the operation, including fuel and aircrew labour costs. In addition, test instrumentation that requires integration into the aircraft must be selected carefully to avoid unnecessary delays in obtaining the airworthiness certification for the modified test aircraft.

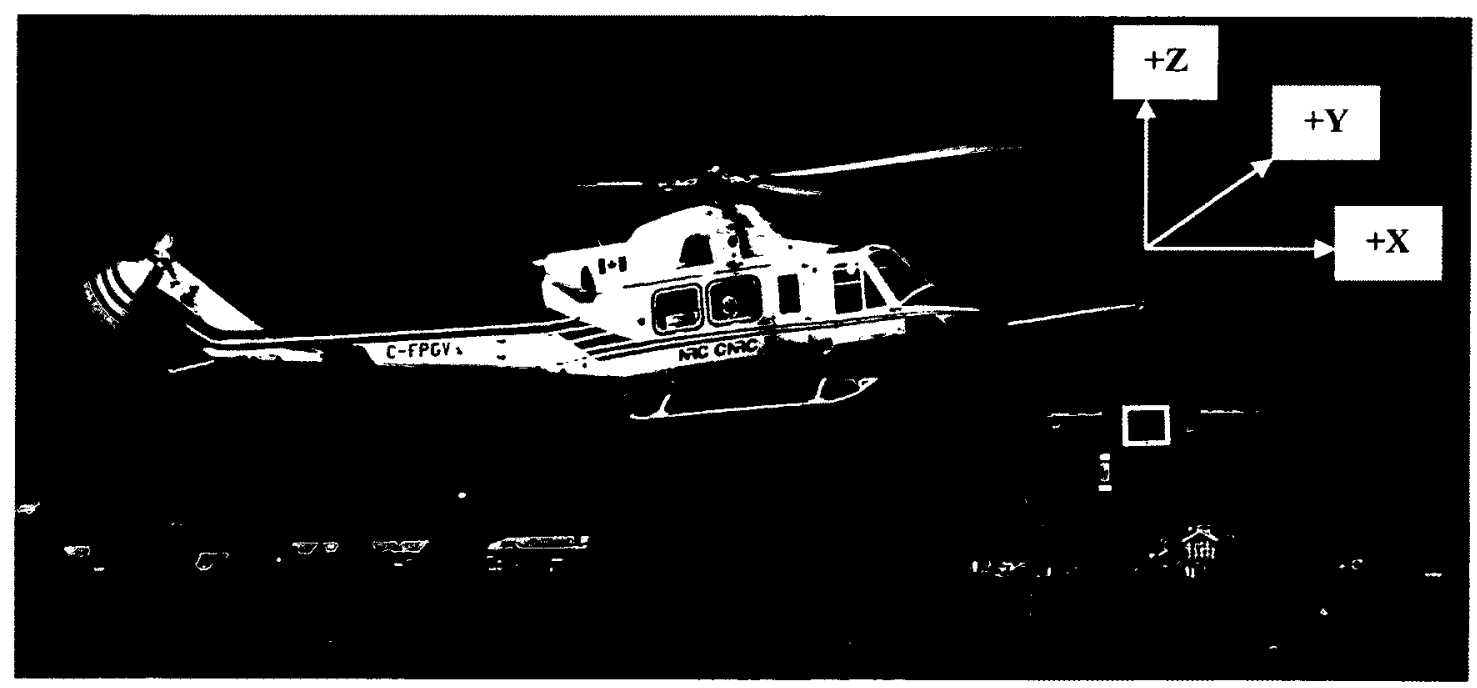

Figure 2.1: Principal directions on the NRC Bell-412 helicopter 


\subsubsection{Flight Test Helicopter}

The Bell-412 helicopter operated by the NRC, shown in Figure 2.1, was selected as the flight test aircraft. The Bell-412 helicopter was selected primarily because it is the civilian version of the $\mathrm{CH}-146$ aircraft used by the Canadian military which has been the subject of reported injuries, pain and discomfort by the aircrew. The Bell-412 is a utility helicopter with a four bladed main rotor system manufactured by Bell Helicopter Textron Canada Inc at its Mirabel plant. The general characteristics and performance specification of the Bell-412 are identified below [89].

\section{General characteristics}

Crew: 1-2 pilots

Capacity: up to 13 passengers

Length: $17.1 \mathrm{~m}$

Rotor diameter: $14.0 \mathrm{~m}$

Empty weight: $3,079 \mathrm{~kg}$

Maximum takeoff weight: $5,397 \mathrm{~kg}$

Power plant: Two Pratt \& Whitney Canada PT6T-3BE turboshafts engine

\section{Performance}

Maximum speed: $259 \mathrm{~km} / \mathrm{h}(140 \mathrm{kts})$

Cruise speed: $226 \mathrm{~km} / \mathrm{h}(122 \mathrm{kts})$

Range: $745 \mathrm{~km}$ (402 nmi)

Service ceiling: $6,096 \mathrm{~m}$

The NRC Bell-412 used for the flight tests is configured as a research aircraft employed in projects on airborne simulation, handling qualities, advanced controls, active controls and pilot-vehicle interfaces. It has been outfitted with advanced technology that makes it an ideal platform to perform research in a variety of areas such as digital fly-bywire control systems, precise guidance and navigation, advanced pilot-vehicle interfaces such as smart displays, helmet-mounted displays et cetera. This NRC Bell-412 helicopter 
is flown under an experimental airworthiness certification which allows airworthiness personnel at NRC rather than Transport Canada to approve modification to the aircraft, saving significant time and avoiding cumbersome bureaucracy for researchers.
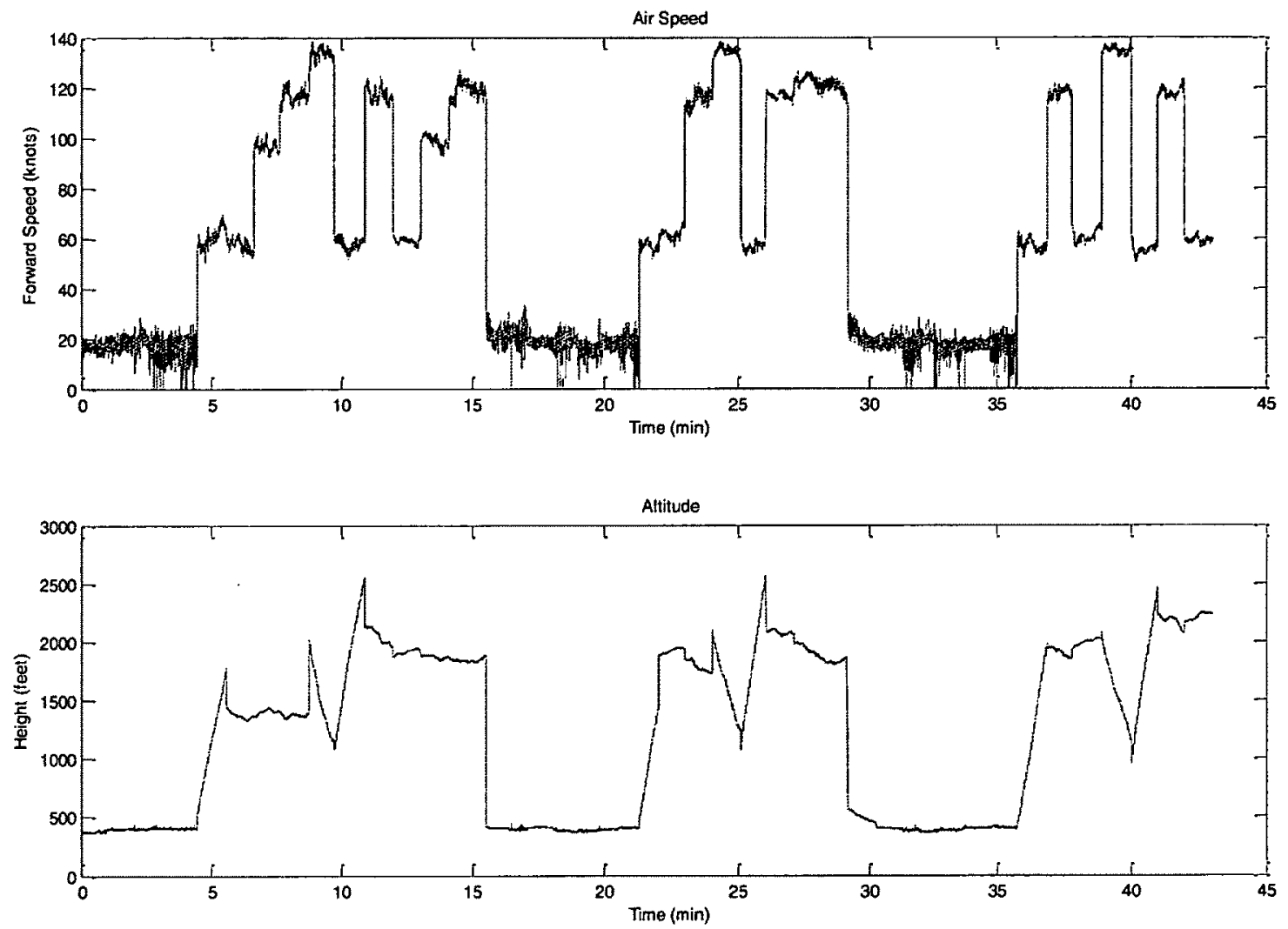

Figure 2.2: Airspeed and Altitude vs. Time for the Test Flight

\subsubsection{Flight Profile}

The flight plan included a wide range of flight conditions such as ground, hover, translational lift, forward flight at $60 \mathrm{kts}, 120 \mathrm{kts}$ and $140 \mathrm{kts}$, in order to identify the variation in the vibration profiles during a typical flight. The cruise condition for the Bell-412 helicopter is $120 \mathrm{kts}$, while $140 \mathrm{kts}$ was achieved through a sharp dive. A minimum of two data points were taken for each flight condition to verify repeatability. Each data point lasted 1 minute while the aircraft maintained the specified flight 
condition. The test flight was conducted on November 27, 2007 and vibration data from all flight conditions were acquired successfully. The airspeed and altitude attained during the test are shown in Figure 2.2. In general, the wind speed was $8 \mathrm{kts}$ from 290 degrees.

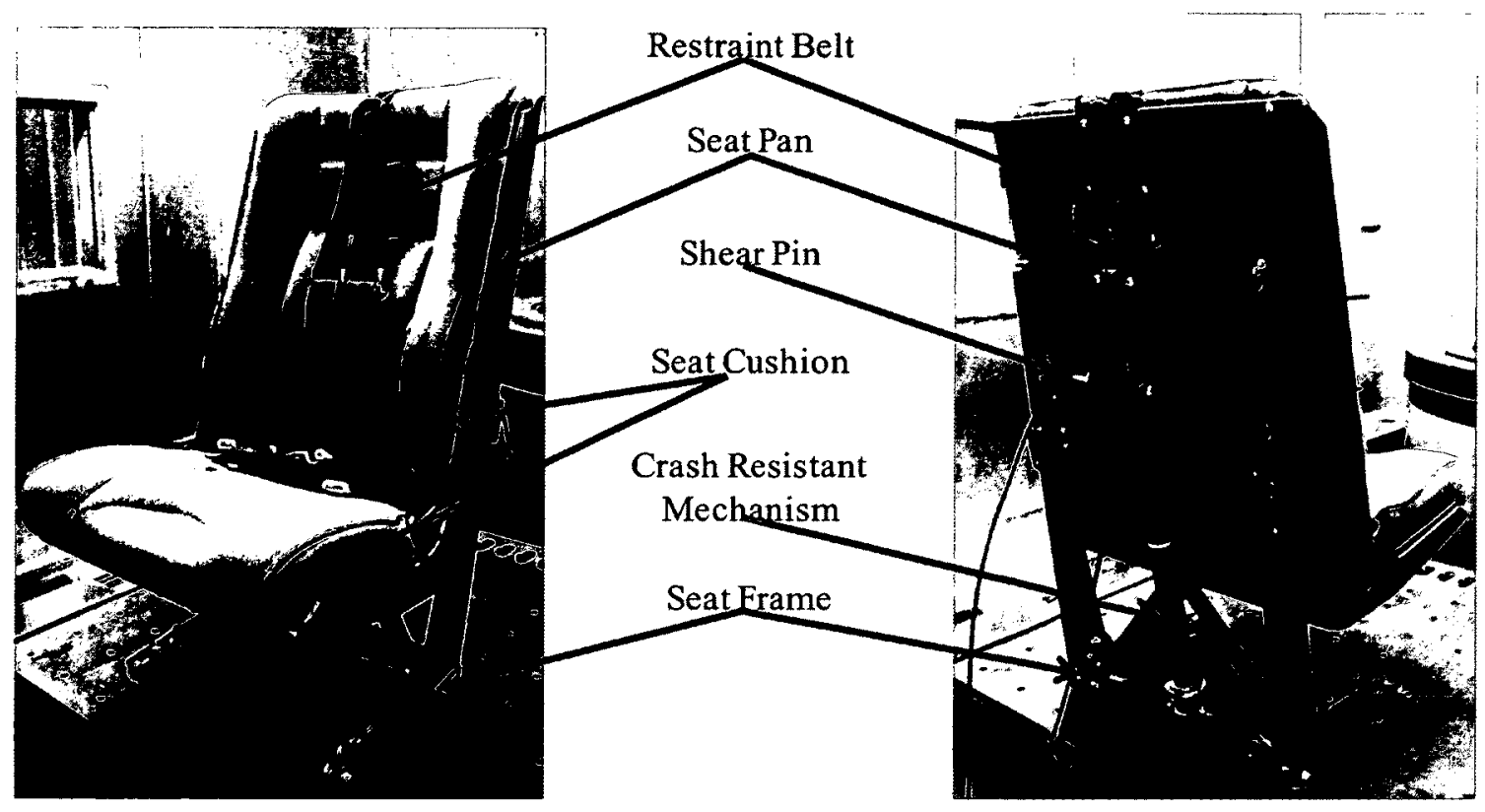

Figure 2.3: Bell-412 aircrew seat

\subsubsection{Aircrew Seat}

The standard Bell-412 non-armoured energy absorption seat shown in Figure 2.3 was used during the flight tests. This seat is composed of a pan made out of stiff composite material that slides on guide rails of the $\mathrm{L}$ shaped frame installed to the helicopter floor. The cushions at the bottom and the back that interface with the aircrew are installed to the seat pan. The seat is attached to the frame through the cylindrical crash resistant mechanism located in the center rear of the seat. The loads generated from the seat are transferred to the frame through a shear pin located on the top of the energy absorption tube. The shear pin was pre-engineered with a notch and the pin is expected to break in the case of a crash, allowing the seat base and pilot to slide downwards along the guide rails. The crushing materials installed inside the crash resistant mechanism was designed 

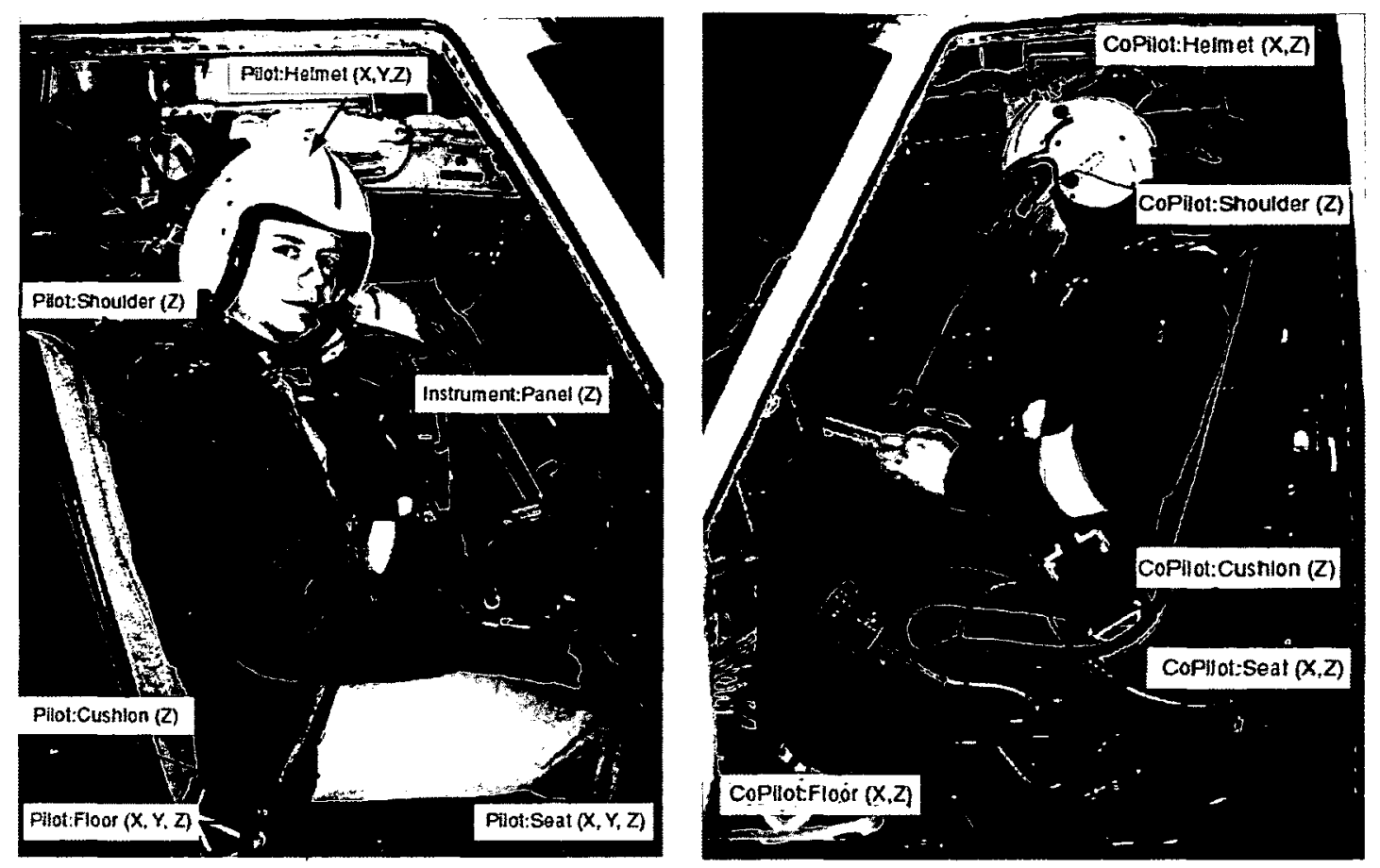

Figure 2.4: Accelerometer locations on the pilot and co-pilot

to absorb the crash impact energy and provide the required impact protection to the aircrew. Two seats were installed for both the pilot location on the right side and the copilot location on the left side of the aircraft. Each seat weighed approximately $50 \mathrm{lbs}$. The test pilot represented a $50^{\text {th }}$ percentile adult male and the co-pilot represented a $95^{\text {th }}$ percentile adult male in terms of height and weight.

\subsubsection{Instrumentation Setup}

The NRC Bell-412 helicopter is a specially configured test helicopter integrated with various flight sensors and an onboard data acquisition system. To characterize the aircrew vibration during flight, it was important to identify the vibration profile along the transmission path from the helicopter floor to the helmet while the pilot is seated. Both pilot as well as co-pilot locations were instrumented with accelerometers to measure the vibration experienced by the aircrew during the flight. Therefore, several measurement locations were selected on the aircrew, in addition to locations on the fuselage and the seat structure. Locations of accelerometers, namely, floor under the seat, bottom of the 
seat, top of the cushion, shoulder and helmet, are shown in Figure 2.4. In addition, one accelerometer was installed on the top of the instrument panel.

Table 2.1: Information of accelerometers used in the flight test

\begin{tabular}{|c|c|c|c|c|c|}
\hline $\begin{array}{c}\text { Channel } \\
\text { No }\end{array}$ & $\begin{array}{c}\text { Location } \\
\text { Name }\end{array}$ & $\begin{array}{c}\text { Accelerometer } \\
\text { Model }\end{array}$ & $\begin{array}{c}\text { Serial } \\
\text { No }\end{array}$ & $\begin{array}{c}\text { Sensor } \\
\text { Sensitivity }\end{array}$ & $\begin{array}{c}\text { Installed } \\
\text { Direction }\end{array}$ \\
\hline 1 & Pilot Helmet & PCB 357C10 & 8704 & $1.79 \mathrm{pC} / \mathrm{g}$ & $\mathrm{Z}$ \\
\hline 2 & Pilot Shoulder & PCB 357C10 & 5563 & $1.48 \mathrm{pC} / \mathrm{g}$ & $\mathrm{Z}$ \\
\hline 3 & Pilot Cushion & PCB 357C10 & 6786 & $1.63 \mathrm{pC} / \mathrm{g}$ & $\mathrm{Z}$ \\
\hline 4 & Pilot Seat & PCB 357C10 & 6787 & $1.54 \mathrm{pC} / \mathrm{g}$ & $\mathrm{Z}$ \\
\hline 5 & Pilot Floor & PCB 357C10 & 8701 & $1.67 \mathrm{pC} / \mathrm{g}$ & $\mathrm{Z}$ \\
\hline 6 & Pilot Helmet & PCB 357C10 & 11668 & $1.55 \mathrm{pC} / \mathrm{g}$ & $\mathrm{X}$ \\
\hline 7 & Pilot Helmet & PCB 357C10 & 8691 & $1.80 \mathrm{pC} / \mathrm{g}$ & $\mathrm{Y}$ \\
\hline 8 & Pilot Seat & PCB 357C10 & 11846 & $1.40 \mathrm{pC} / \mathrm{g}$ & $\mathrm{X}$ \\
\hline 9 & Pilot Seat & PCB 357C10 & 11669 & $1.61 \mathrm{pC} / \mathrm{g}$ & $\mathrm{Y}$ \\
\hline 10 & Pilot Floor & PCB 357C10 & 12061 & $1.46 \mathrm{pC} / \mathrm{g}$ & $\mathrm{X}$ \\
\hline 11 & Pilot Floor & PCB 357C10 & 8694 & $1.85 \mathrm{pC} / \mathrm{g}$ & $\mathrm{Y}$ \\
\hline 12 & Instrument Panel & PCB 357C10 & 8697 & $1.63 \mathrm{pC} / \mathrm{g}$ & $\mathrm{Z}$ \\
\hline 13 & Co-Pilot Helmet & PCB 352C22 & 58327 & $9.20 \mathrm{mV} / \mathrm{g}$ & $\mathrm{Z}$ \\
\hline 14 & Co-Pilot Shoulder & PCB 352C22 & 58328 & $9.17 \mathrm{mV} / \mathrm{g}$ & $\mathrm{Z}$ \\
\hline 15 & Co-Pilot Cushion & PCB 352C22 & 58325 & $8.77 \mathrm{mV} / \mathrm{g}$ & $\mathrm{Z}$ \\
\hline 16 & Co-Pilot Seat & PCB 352C22 & 58326 & $8.75 \mathrm{mV} / \mathrm{g}$ & $\mathrm{Z}$ \\
\hline 17 & Co-Pilot Floor & PCB 352C22 & 39250 & $9.41 \mathrm{mV} / \mathrm{g}$ & $\mathrm{Z}$ \\
\hline 18 & Co-Pilot Helmet & PCB 352C22 & 88189 & $10.00 \mathrm{mV} / \mathrm{g}$ & $\mathrm{X}$ \\
\hline 19 & Co-Pilot Seat & PCB 352C22 & 88190 & $10.17 \mathrm{mv} / \mathrm{g}$ & $\mathrm{X}$ \\
\hline 20 & Co-Pilot Floor & PCB 352C22 & 88191 & $9.62 \mathrm{mV} / \mathrm{g}$ & $\mathrm{X}$ \\
\hline
\end{tabular}

The principal axis directions in the helicopter selected for the test are shown in Figure 2.1. The floor, seat and the helmet locations for the pilot included accelerometers in all 3 directions while those locations for the co-pilot measured only in $\mathrm{X} \& \mathrm{Z}$ directions. The vibration at the remaining locations was measured only in the $\mathrm{Z}$ direction. The vibration response at the selected locations and directions was measured using 20 high sensitivity miniature accelerometers. Accelerometers installed on the structure were bonded with epoxy while the accelerometers that were placed on the aircrew were affixed with wax to facilitate ease of removal. It is a flight safety 
requirement to ensure that instrumentation such as accelerometers placed on the aircrew can be removed easily during any emergency. The high sensitivity of the accelerometers was ideal to obtain good signal-to-noise ratio measurements even when the response amplitudes were relatively low in the presence of relatively high background noise that exists in the environment. Details of the accelerometers used for the test is shown in Table 2.1. Prior to the flight test, all the accelerometers were calibrated with a $\mathrm{B} \& \mathrm{~K}$ 4294 Exciter to verify accuracy and ensure reliability.

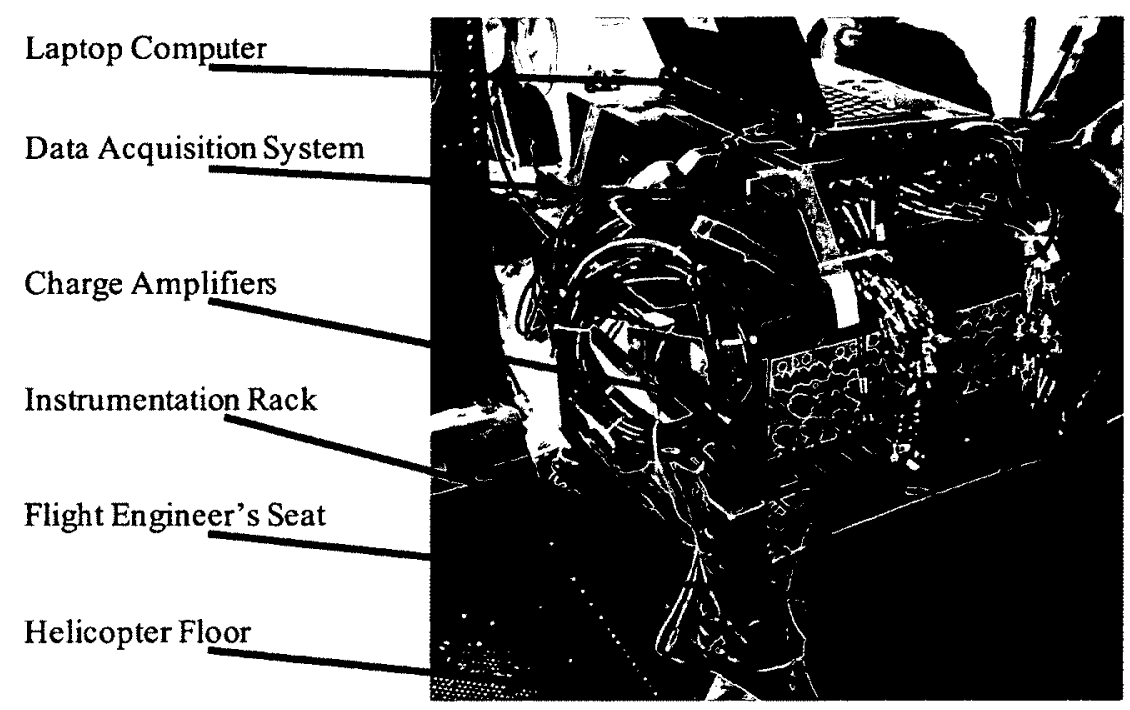

Figure 2.5: Instrumentation setup in the NRC Bell-412 helicopter

The LMS SCADAS 305 mobile front-end data acquisition system was configured for simultaneous data recording of the 20 accelerometer channels during the flight test [90]. All PCB 352C22 accelerometers were connected directly to the signal conditioning cards of the LMS data acquisition system, while each of the PCB 357C10 charge type accelerometers were connected through a B \& K 2635 model charge amplifiers prior to signal acquisition. The data acquisition hardware, charge amplifiers as well as the laptop computer used for data recording were installed in the aircraft rack designated for research equipment. The instrumentation setup in the NRC Bell-412 aircraft cabin is shown in Figure 2.5. Once all the instrumentation was installed, airworthiness personnel 
approved the installation of non-standard equipment in the aircraft based on established safety and operational regulations. The sampling frequency of $2 \mathrm{kHz}$ was used in the data acquisition system, which is approximately 10 times above the maximum frequency of interest in order to ensure the high quality of the acquired data. The time domain data were recorded digitally and simultaneously analyzed in the frequency domain to reveal the real-time vibration spectrum from each accelerometer using the onboard laptop computer. For each flight condition, the vibration data recorder was switched on manually by the test engineer once the pilot verified that the aircraft as well as the aircrew were at the pre-determined test condition.

The commercially available LMS Test.Lab Spectral Testing software package was used to record the data on the laptop [91]. The Flat-Top windowing function was used for the Fast Fourier Transform (FFT) to obtain accurate amplitude from the sinusoidal content in the recorded signal. This windowing function was applied to each data block used for the FFT calculation to avoid discontinuities, which resulted in better spectral resolution. In addition, a frequency resolution of $0.25 \mathrm{~Hz}$ was selected for the data analysis in order to accurately estimate the peak acceleration at each rotor harmonic. To reduce the noise in the measured data, 15 linear averages were taken during each test run which lasted only 1 minute.

\subsection{Flight Test Results and Discussions}

The flight test was conducted successfully using the NRC Bell-412 helicopter and the vibration data were recorded for a number of typical flight conditions. The flight test demonstrated that the helicopter aircrew were exposed to high levels of whole body vibration during flight. The overall vibration levels measured in the time domain were used to identify the level of comfort based on a commonly used comfort metric identified in ISO 2631 standard [92]. In addition, vibration data was analyzed in the frequency domain to determine the vibratory spectrum in order to design the adaptive seat mount to counteract the critical modes of vibration. Details of the analyses performed on the vibration data acquired during the flight test are discussed below. 
Table 2.2: Measured g-rms vibration levels for the pilot locations

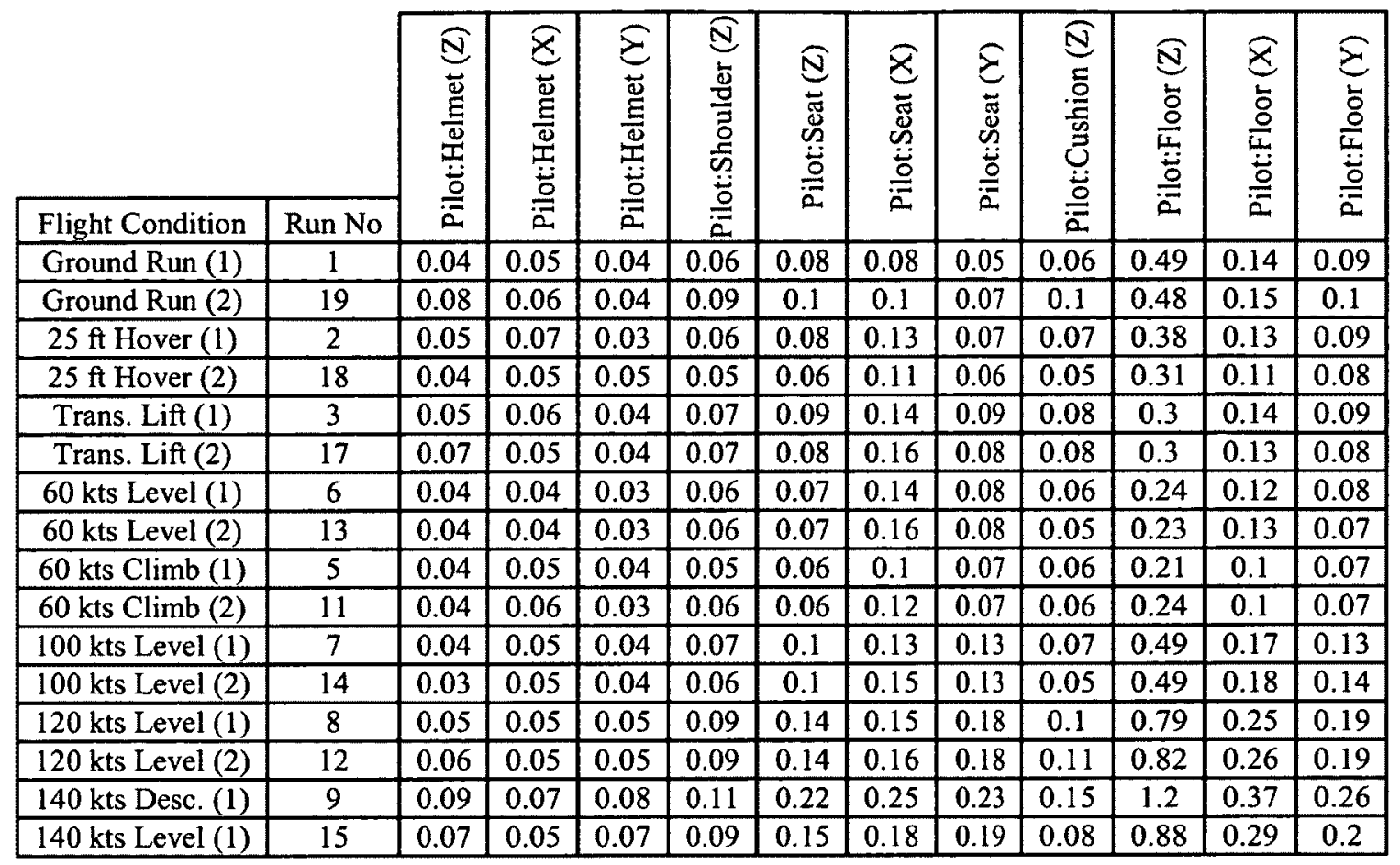

Table 2.3: Measured g-rms vibration levels for the co-pilot locations

\begin{tabular}{|c|c|c|c|c|c|c|c|c|c|c|}
\hline & \multirow{3}{*}{ 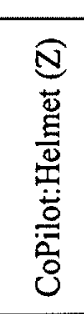 } & \multirow{3}{*}{ 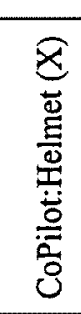 } & \multirow{3}{*}{ 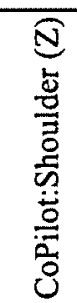 } & \multirow{3}{*}{ 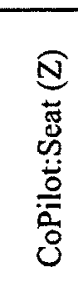 } & \multirow{3}{*}{ 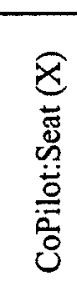 } & \multirow{3}{*}{ 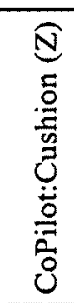 } & & & \\
\hline & & & & & & & & \multirow[t]{2}{*}{ 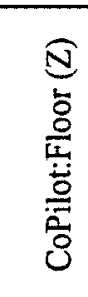 } & \multirow[t]{2}{*}{ 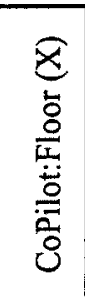 } & \multirow[t]{2}{*}{ 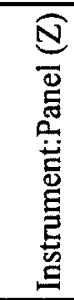 } \\
\hline Flight Condition & Run No & & & & & & & & & \\
\hline Ground Run (1) & 1 & 0.05 & 0.07 & 0.08 & 0.06 & 0.08 & 0.22 & 0.29 & 0.07 & 0.23 \\
\hline Ground Run (2) & 19 & 0.07 & 0.1 & 0.1 & 0.07 & 0.11 & 0.28 & 0.25 & 0.08 & 0.27 \\
\hline $25 \mathrm{ft} \mathrm{Hover}(1)$ & 2 & 0.07 & 0.07 & 0.1 & 0.08 & 0.14 & 0.25 & 0.25 & 0.1 & 0.28 \\
\hline $25 \mathrm{ft} \mathrm{Hover} \mathrm{(2)}$ & 18 & 0.06 & 0.07 & 0.08 & 0.06 & 0.13 & 0.22 & 0.23 & 0.09 & 0.21 \\
\hline Trans. Lift (1) & 3 & 0.06 & 0.06 & 0.1 & 0.09 & 0.15 & 0.26 & 0.26 & 0.12 & 0.32 \\
\hline Trans. Lift (2) & 17 & 0.08 & 0.07 & 0.09 & 0.08 & 0.16 & 0.25 & 0.22 & 0.12 & 0.26 \\
\hline 60 kts Level (1) & 6 & 0.07 & 0.06 & 0.07 & 0.08 & 0.13 & 0.17 & 0.2 & 0.11 & 0.31 \\
\hline 60 kts Level (2) & 13 & 0.05 & 0.05 & 0.07 & 0.09 & 0.14 & 0.16 & 0.2 & 0.12 & 0.3 \\
\hline 60 kts Climb (1) & 5 & 0.07 & 0.07 & 0.08 & 0.07 & 0.12 & 0.23 & 0.22 & 0.09 & 0.19 \\
\hline 60 kts Climb (2) & 11 & 0.05 & 0.07 & 0.07 & 0.06 & 0.12 & 0.21 & 0.2 & 0.09 & 0.17 \\
\hline $100 \mathrm{kts}$ Level (1) & 7 & 0.08 & 0.06 & 0.1 & 0.09 & 0.15 & 0.18 & 0.29 & 0.12 & 0.36 \\
\hline 100 kts Level (2) & 14 & 0.08 & 0.06 & 0.09 & 0.09 & 0.16 & 0.16 & 0.29 & 0.13 & 0.38 \\
\hline $120 \mathrm{kts}$ Level (1) & 8 & 0.09 & 0.07 & 0.13 & 0.13 & 0.17 & 0.24 & 0.46 & 0.14 & 0.47 \\
\hline $120 \mathrm{kts}$ Level (2) & 12 & 0.11 & 0.07 & 0.12 & 0.12 & 0.18 & 0.22 & 0.43 & 0.14 & 0.47 \\
\hline 140 kts Desc. (1) & 9 & 0.11 & 0.06 & 0.17 & 0.15 & 0.25 & 0.23 & 0.59 & 0.22 & 0.64 \\
\hline 140 kts Level (1) & 15 & 0.12 & 0.07 & 0.14 & 0.14 & 0.18 & 0.26 & 0.49 & 0.15 & 0.52 \\
\hline
\end{tabular}




\subsubsection{Overall Vibration Level}

In the past, a variety of methods have been used to determine the health risk and comfort of an occupant of a vehicle based on the level of vibration exposure on a variety of vehicles, including buses, aircraft, as well as cars [93]. The simplest and the most commonly used method is the root mean square (rms) acceleration because it evaluates the overall magnitude of vibration with high frequency content. The rms acceleration is considered as the total energy across the entire frequency range of the vibration input. The rms value of a signal is defined as the normalized second statistical moment of the signal or the standard deviation of the signal as:

$$
a=\sqrt{\frac{1}{T} \int_{0}^{T}(x(t)-\bar{x})^{2} d t}
$$

where, $T$ is the length of operating time and $\bar{x}$ is the mean value of the signal. For sampled or digitized signals with $N$ samples, rms of the signal is defined as:

$$
a=\sqrt{\frac{1}{N} \sum_{n=0}^{N-1}(x(n)-\bar{x})^{2}}
$$

with

$$
\bar{x}=\frac{1}{N} \sum_{n=0}^{N-1} x(n)
$$

The overall $\mathrm{rms}$ acceleration levels measured in $\mathrm{g}$ for the pilot and co-pilot locations for each corresponding flight condition are calculated and shown in Table 2.2 and Table 2.3, respectively. As expected, the helicopter structural vibrations measured in terms of g-rms increased with forward airspeed. Similarly, the vibration measured on the aircrew, specifically at the helmet location, also increased with forward speed. In general, the measured acceleration shows that the floor vibration at the co-pilot's location is lower than that of the pilot's location. However, the helmet vibration measured at the 
co-pilot is greater than the pilot. This variation could be due to the differences in the aircrew weight, tightness of the safety belt restraint harness, activity and posture. For example, while the pilot was flying the helicopter with both hands and feet on the controls, the co-pilot was working on a laptop acquiring data. These differences may account for the variation in vibration levels measured at the pilot's and co-pilot's locations.

The g-rms values show that the overall vibration levels varied substantially depending on flight conditions for each measurement location. In order to further analyze the effect of vibration on the aircrew, ISO 2631 was applied to assess the level of comfort, perception and health risk based on the flight test data.

\subsubsection{Vibration Assessment Based on ISO 2631 Standard}

The ISO 2631 standard is the most widely used metric to assess the effect of the whole body vibration exposure as it pertains to vehicles [94]. In general, the ISO 2631 standard recommends that the human operator and vehicle interface vibration be measured on locations and directions shown in Figure 2.6. This standard also specifies a number of frequency weighting functions to be applied between $0.01 \mathrm{~Hz}$ to $80 \mathrm{~Hz}$ to acceleration measurement to account for the human body sensitivity at specific frequencies [92].

- $\mathrm{W}_{\mathrm{k}}$ for vertical vibrations, Z-direction

- $\mathrm{W}_{\mathrm{d}}$ for horizontal vibrations, $\mathrm{X}$ - and $\mathrm{Y}$-directions

- $\mathrm{W}_{\mathrm{c}}$ for seat back vibration, $\mathrm{X}$-direction

- $\mathrm{W}_{\mathrm{e}}$ for rotational

- $\mathrm{W}_{\mathrm{j}}$ for head vibration

- $\mathrm{W}_{\mathrm{f}}$ for motion sickness

Details of these weighting functions are available in the ISO 2631 standard [92]. Based on the measured vibration, this standard identifies three different methods to assess whole-body vibration and evaluate its effects on the operator as described in detail below. 


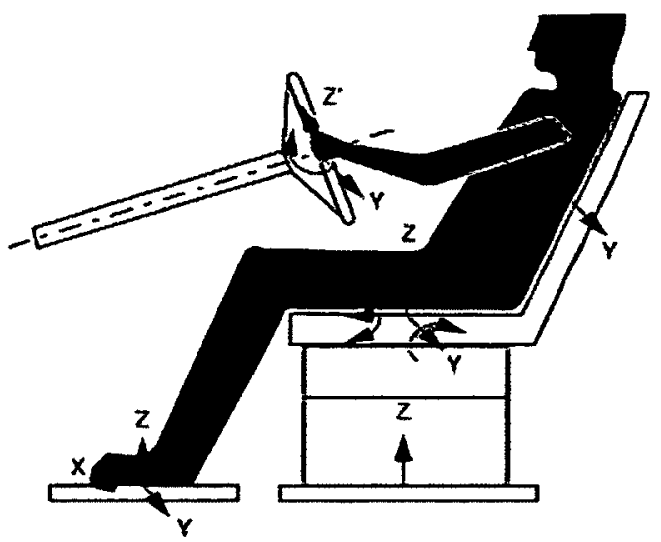

Figure 2.6: ISO 2631 Recommended Acceleration Measurements [92]

\subsubsection{Weighted rms Acceleration Method}

Weighted rms acceleration is calculated by multiplying each of the measured acceleration with the appropriate frequency weighting function specified in the ISO 2631 standard. The weighted rms calculation method is used to express the acceleration in meters per second squared $\left(\mathrm{m} / \mathrm{s}^{2}\right)$ for translational vibration.

$$
a_{w}=\sqrt{\sum_{i}^{N}\left(W_{i} a_{i}\right)^{2}}
$$

where, $a_{w}$ is the frequency weighted acceleration, $W_{i}$ is the weighting factor based on ISO 2631 standard and $a_{i}$ is the acceleration for the $\mathrm{i}^{\text {th }}$ frequency spectrum line. The total is determined by the vibration measured in the orthogonal directions as follows.

$$
a_{v}=\sqrt{k_{x}^{2} a_{w x}^{2}+k_{y}^{2} a_{w y}^{2}+k_{z}^{2} a_{w z}^{2}}
$$

where, $a_{w x}, a_{w y}, a_{w z}$ are weighted rms accelerations for orthogonal $\mathrm{x}, \mathrm{y}, \mathrm{z}$ axes, respectively. The multiplying factors are $k_{x}=1.4$ for health and 1.0 for comfort, $k_{y}=1.4$ for health and 1.0 for comfort and $k_{z}=1.0$ for both health and comfort. Based on the rms 
acceleration, an assessment of the effects of vibration on the level of comfort is provided in the standard as shown in Table 2.4 .

Table 2.4: rms acceleration ranges for level of comfort [92]

\begin{tabular}{|c|c|}
\hline Level of Comfort & rms acceleration $\left.\mathbf{~} \mathbf{m} / \mathbf{s}^{\mathbf{2}}\right)$ \\
\hline Not uncomfortable & $<0.315$ \\
\hline A little uncomfortable & $0.315-0.63$ \\
\hline Fairly uncomfortable & $0.5-1$ \\
\hline Uncomfortable & $0.8-1.6$ \\
\hline Very uncomfortable & $1.25-2.5$ \\
\hline Extremely uncomfortable & $>2$ \\
\hline
\end{tabular}

In addition, ISO 2631 standard stipulates a health guidance criterion indicated as a "caution zone" for the range of 4-8 hr exposure for weighted rms acceleration levels between $0.5 \mathrm{~m} / \mathrm{s}^{2}$ and $1.2 \mathrm{~m} / \mathrm{s}^{2}$. It is assumed that exposure to vibrations below the lower limit of this zone is "safe" while exposure to vibration levels above the upper limit is "not safe". The standard also indicates that this basic evaluation method may underestimate the effects of the vibration if the vibration signal consists of occasional shocks and harmonics. Therefore, two additional evaluation methods, namely, the maximum transient vibration value and the vibration dose value have been presented in the ISO 2631 standard.

\subsubsection{Maximum Transient Vibration Value}

The maximum transient vibration value (MTVV) is calculated based on the running rms evaluation method. This is a different method than the basic weighted rms evaluation method because it takes into account shocks and transient vibration through the use of a short integration time constant. MTVV is calculated as follows:

$$
M T V V=\max \left(\sqrt{\frac{1}{\tau} \int_{t_{0}-\tau}^{t_{0}}\left[a_{w}(t)\right]^{2} d t}\right)
$$


where, $a_{w}(t)$ is the instantaneous frequency-weighted acceleration, $\tau$ is the integration time; $t$ is the integration variable and $t_{0}$ is instantaneous time. However, no guidance for acceptable limits of the MTVV is given in the ISO 2631 standard [95].

\subsubsection{Vibration Dose Value}

The Vibration Dose Value (VDV) method is more sensitive to peaks than the rms or MTVV methods by using the fourth power instead of the second power of the acceleration time history as the basis for averaging. The VDV is expressed in meters per second to the power $1.75\left(\mathrm{~m} / \mathrm{s}^{1.75}\right)$ to evaluate human perception to vibration and it is calculated as follows:

$$
V D V=\left\{\int_{0}^{T}\left[a_{w}(t)\right]^{4} d t\right\}^{1 / 4}
$$

where, $a_{w}(t)$ is the instantaneous frequency-weighted acceleration, $T$ is the duration of the measurement and $t$ is the integration variable.

Although ISO 2631 does not provide references to levels of perception ranges based on VDV, recent research has suggested ranges for the VDV measurements be based on level of perception as shown in Table 2.5 [96]. These levels of perception were used to determine aircrew perception based on the VDV calculated for the flight tests data.

Table 2.5: VDV ranges for level of perception [96]

\begin{tabular}{|c|c|}
\hline Level of Perception & VDV $\left(\mathbf{m} / \mathbf{s}^{\mathbf{1 . 7 5}}\right)$ \\
\hline Reasonable & $<0.66$ \\
\hline Disturbing & $0.66-2.38$ \\
\hline Unacceptable & $2.38-4.64$ \\
\hline Panic & $>4.64$ \\
\hline
\end{tabular}




\subsubsection{Assessment of Aircrew Health, Comfort and Perception}

The three different methods recommenced by ISO 2631 standard were used to assess the whole-body vibration of the aircrew recorded during flight tests. Only the accelerometer data at the cushion location was used for calculation because this standard requires the acceleration measured at the occupant-machine interface [92]. Although the standard requires acceleration in all three orthogonal directions to be measured and consolidated using vector addition, this flight test only measured vertical Z-direction vibration. It is reasonable to assume that the $\mathrm{Z}$-direction vibration is the most dominant at the cushion interface while the effects of lateral and transverse vibration could be neglected [97]. The Human Body Vibration module of the commercially available LMS Test.Lab data analysis software package was used to calculate the rms, MTVV and VDV based on measured acceleration on the cushion. The level of comfort, health guidance and level of perception based on rms, MTVV and VDV assessed for the pilot and co-pilot for each corresponding flight condition is shown in Table 2.6 and Table 2.7, respectively.

The level of comfort assessed for the pilot clearly shows that most flight conditions are considered "uncomfortable" while the $140 \mathrm{kts}$ condition is rated as "very uncomfortable". In addition, the health guidance rates several flight conditions as "caution" while the $140 \mathrm{kts}$ condition is rated as "not safe". However, the vibration assessment for the co-pilot shows a significantly more severe vibration environment with several "extremely uncomfortable" flight conditions. Furthermore, all flight conditions are rated as "not safe" based on the health guidance for the 4-8 hour exposure time. As stated previously, the variation in pilot and co-pilot vibration assessment may be due to the weight, tightness of their harness restraint, and posture among other factors. For example, the tested pilot represented a $50^{\text {th }}$ percentile male while the co-pilot represented a $95^{\text {th }}$ percentile male performing vastly different activities. These significant differences may explain the variation in the ISO 2631 assessment based on measured vibration for the pilot and co-pilot. Although this data was sufficient for the current purpose of measuring aircrew vibration to develop an adaptive seat mount, it is recommended that a program of thorough flight tests be performed with a larger sample size to increase the statistical significance of the data. 
Table 2.6: Measured vibration of the pilot evaluated based ISO 2631 standard

\begin{tabular}{|c|c|c|c|c|c|c|}
\hline $\begin{array}{c}\text { Flight } \\
\text { Condition }\end{array}$ & $\begin{array}{c}\mathbf{r m s} \\
\left(\mathbf{m} / \mathbf{s}^{2}\right)\end{array}$ & $\begin{array}{c}\text { MTVV } \\
\left(\mathbf{m} / \mathbf{s}^{2}\right)\end{array}$ & $\begin{array}{c}\text { VDV } \\
\left(\mathbf{m} / \mathbf{s}^{\mathbf{1 . 7 5}}\right)\end{array}$ & $\begin{array}{c}\text { Level of } \\
\text { Comfort }\end{array}$ & $\begin{array}{c}\text { Health } \\
\text { Guidance }\end{array}$ & $\begin{array}{c}\text { Level of } \\
\text { Perception }\end{array}$ \\
\hline Ground Run (1) & 0.51 & 0.78 & 1.96 & A Little Uncomfortable & Safe & Disturbing \\
\hline Ground Run (2) & 0.88 & 1.28 & 3.04 & Uncomfortable & Caution & Unacceptable \\
\hline 25 ft Hover (1) & 0.58 & 8.83 & 1.57 & Fairly Uncomfortable & Caution & Disturbing \\
\hline 25 ft Hover (2) & 0.39 & 0.49 & 1.37 & A Little Uncomfortable & Caution & Disturbing \\
\hline Trans. Lift (1) & 0.52 & 0.59 & 1.96 & A Little Uncomfortable & Caution & Disturbing \\
\hline Trans. Lift (2) & 0.52 & 0.78 & 1.77 & A Little Uncomfortable & Safe & Disturbing \\
\hline 60kt Level (1) & 0.39 & 0.49 & 1.37 & Not Uncomfortable & Safe & Disturbing \\
\hline 60kt Level (2) & 0.41 & 0.48 & 1.67 & A Little Uncomfortable & Safe & Disturbing \\
\hline 60kt Climb (1) & 0.49 & 0.59 & 1.47 & A Little Uncomfortable & Caution & Disturbing \\
\hline 60kt Climb (2) & 0.39 & 0.49 & 1.37 & A Little Uncomfortable & Safe & Disturbing \\
\hline 100kt Level (1) & 0.39 & 0.59 & 1.47 & A Little Uncomfortable & Safe & Disturbing \\
\hline 100kt Level (2) & 0.29 & 0.39 & 1.57 & Not Uncomfortable & Safe & Disturbing \\
\hline 120kt Level (1) & 0.59 & 0.98 & 2.38 & Fairly Uncomfortable & Caution & Unacceptable \\
\hline 120kt Level (2) & 0.59 & 0.88 & 2.45 & Fairly Uncomfortable & Caution & Unacceptable \\
\hline 140kt Desc (1) & 1.18 & 1.37 & 3.43 & Very Uncomfortable & Not Safe & Unacceptable \\
\hline 140kt Level (1) & 0.49 & 0.78 & 2.16 & A Little Uncomfortable & Caution & Disturbing \\
\hline
\end{tabular}

Table 2.7: Measured vibration of the co-pilot evaluated based ISO 2631 standard

\begin{tabular}{|c|c|c|c|c|c|c|}
\hline $\begin{array}{c}\text { Flight } \\
\text { Condition }\end{array}$ & $\begin{array}{c}\text { rms } \\
\left(\mathbf{m} / \mathbf{s}^{\mathbf{2}}\right.\end{array}$ & $\begin{array}{c}\text { MTVV } \\
\left(\mathbf{m} / \mathbf{s}^{\mathbf{2}}\right)\end{array}$ & $\begin{array}{c}\text { VDV } \\
\left(\mathbf{m} / \mathbf{s}^{1.75}\right)\end{array}$ & $\begin{array}{c}\text { Level of } \\
\text { Comfort }\end{array}$ & $\begin{array}{c}\text { Health } \\
\text { Guidance }\end{array}$ & $\begin{array}{c}\text { Level of } \\
\text { Perception }\end{array}$ \\
\hline Ground Run (1) & 2.06 & 2.75 & 7.16 & Very Uncomfortable & Not Safe & Panic \\
\hline Ground Run (2) & 2.75 & 3.73 & 8.53 & Extremely Uncomfortable & Not Safe & Panic \\
\hline 25 ft Hover (1) & 2.35 & 2.84 & 8.00 & Extremely Uncomfortable & Not Safe & Panic \\
\hline 25 ft Hover (2) & 1.86 & 2.45 & 6.57 & Very Uncomfortable & Not Safe & Panic \\
\hline Trans. Lift (1) & 1.77 & 3.04 & 6.08 & Very Uncomfortable & Not Safe & Panic \\
\hline Trans. Lift (2) & 1.86 & 2.55 & 6.08 & Very Uncomfortable & Not Safe & Panic \\
\hline 60kt Level (1) & 1.37 & 1.77 & 4.91 & Uncomfortable & Not Safe & Panic \\
\hline 60kt Level (2) & 1.47 & 1.77 & 5.00 & Uncomfortable & Not Safe & Panic \\
\hline 60kt Climb (1) & 1.96 & 2.55 & 6.28 & Very Uncomfortable & Not Safe & Panic \\
\hline 60kt Climb (2) & 1.77 & 2.06 & 6.28 & Very Uncomfortable & Not Safe & Panic \\
\hline 100kt Level (1) & 1.47 & 2.35 & 5.10 & Uncomfortable & Not Safe & Panic \\
\hline 100kt Level (2) & 1.37 & 1.77 & 5.10 & Uncomfortable & Not Safe & Panic \\
\hline 120kt Level (1) & 1.86 & 2.26 & 6.67 & Very Uncomfortable & Not Safe & Panic \\
\hline 120kt Level (2) & 1.96 & 2.55 & 6.87 & Very Uncomfortable & Not Safe & Panic \\
\hline 140kt Desc (1) & 2.16 & 2.55 & 7.34 & Extremely Uncomfortable & Not Safe & Panic \\
\hline 140kt Level (1) & 2.35 & 2.94 & 8.04 & Extremely Uncomfortable & Not Safe & Panic \\
\hline
\end{tabular}




\subsubsection{Statistical Significance of Data}

It is important to note that the vibration data analyzed for each flight condition was acquired by flying a single Bell-412 aircraft twice at a specified flight condition with a particular pilot and co-pilot. Therefore, it is recommended that more extensive flight tests be conducted by a larger population of pilots and co-pilots as well as by using several aircraft of the same type in order to obtain a more statistically significant set of flight data.

\subsection{Frequency Domain Analysis}

The above assessment based on the commonly used ISO 2631 standard clearly shows the necessity to reduce the level of vibration experienced by helicopter aircrew. Although these metrics provide a general assessment, they are overly simplistic because they do not account for the spectral sensitivity of the human body to vibration. For example, a particular level of vibration may be felt as more or less disruptive, depending on the frequency at which it occurs. Therefore, it is necessary to analyze the measured vibration in the frequency domain. In addition, the design of the adaptive seat mount requires knowledge of the spectral content of the input excitation in order to be effective in counteracting the vibration. Therefore, spectral analysis of the flight tests data was performed in the frequency domain.

\subsubsection{Spectral Analysis}

The recorded vibration data was also analyzed in the frequency domain using spectral analysis to identify harmonics of the main rotor, which is the primary vibratory source acting on the helicopter cabin. In general, the auto-power spectrum, $G_{x x}$, for a given frequency $f$ is defined as

$$
G_{x x}=X(f) \cdot X^{*}(f)
$$


where, $X(f)$ is the FFT of the input signal and $X^{*}(f)$ is the complex conjugate of $X(f)$.

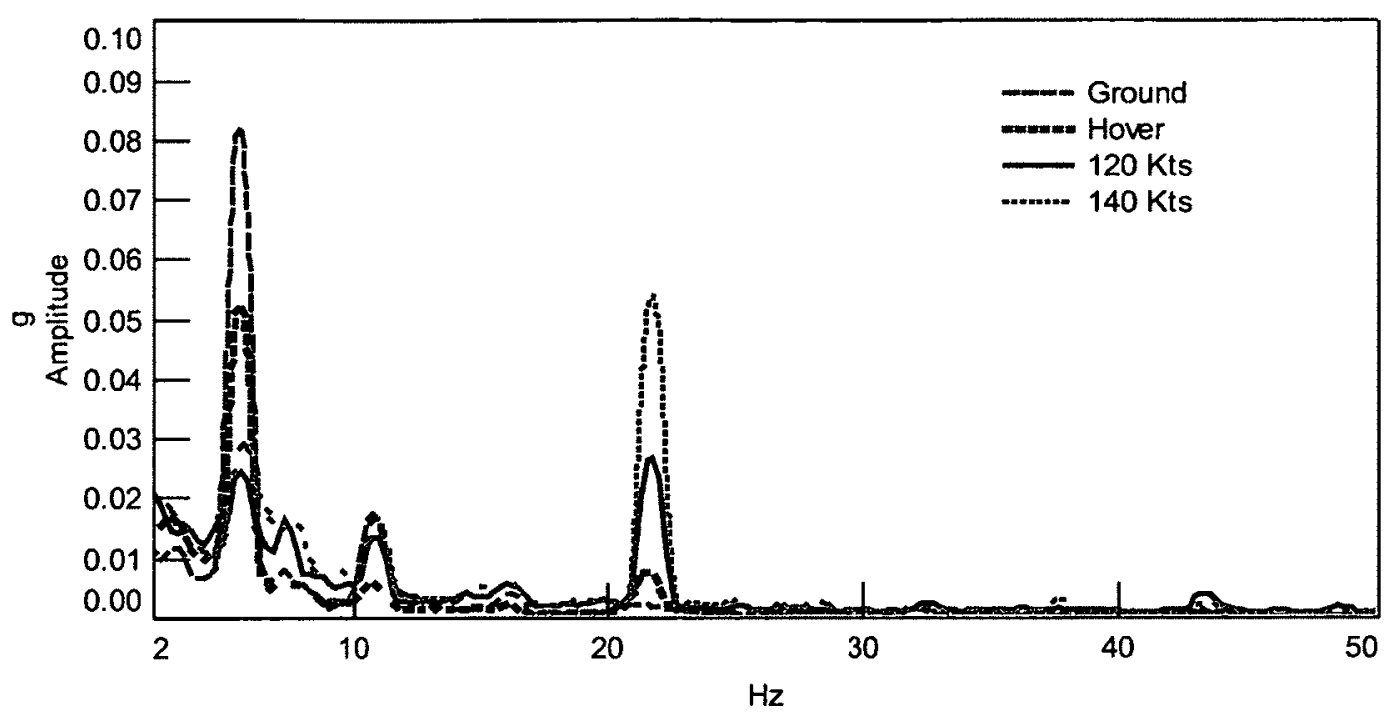

Figure 2.7: Pilot helmet vibration in the $\mathrm{X}$-direction

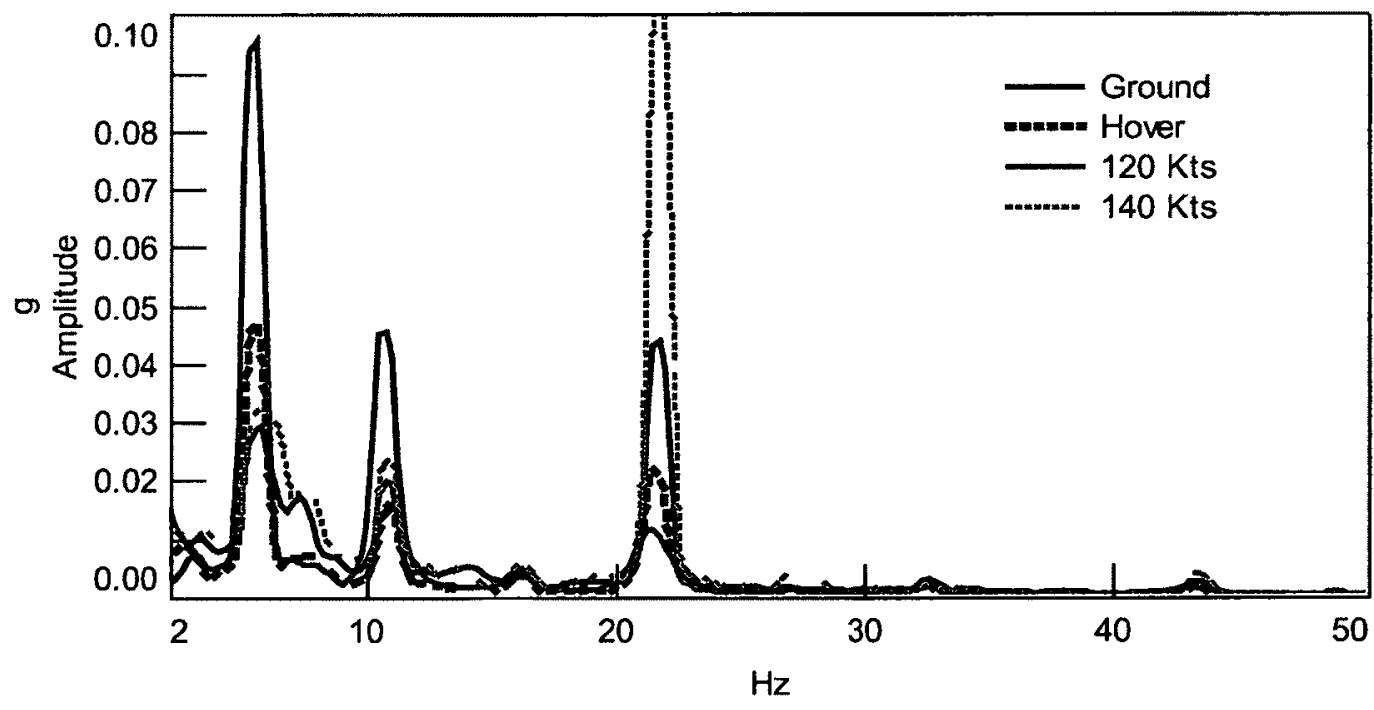

Figure 2.8: Pilot helmet vibration in the Z-direction

The frequency range of interest of the autopower spectra analysis was selected between $1 \mathrm{~Hz}$ and $50 \mathrm{~Hz}$. The power spectrum format was converted to linear spectrum 
in order to extract the amplitude at the harmonic frequencies. Frequency domain analysis of the flight test results showed that the vibration spectrum at the fuselage, seat and pilot body were dominated by the $\mathrm{N} / \mathrm{rev}$ harmonics of the blade passage frequency despite the changes in vibration levels due to various operating conditions. For the floor vibration, the dominant peak was $21.5 \mathrm{~Hz}$, which corresponded to $4 / \mathrm{rev}$ of the rotor passage frequency $(\mathrm{N}=4)$, other peaks included $1 / \mathrm{rev}, 2 / \mathrm{rev}, 6 / \mathrm{rev}$ and $8 / \mathrm{rev}$. Vibration levels measured on the pilot body, including helmet and shoulder locations, also varied with flight conditions. A comparison of $\mathrm{X}$ and $\mathrm{Z}$ direction vibration spectra measured at pilot helmet location at several typical flight conditions are shown in Figure 2.7 and Figure 2.8 , respectively. The autopower spectra of all accelerometers for all flight conditions are provided in Appendix A. In general, the vibration levels increased with flight speed. The worst vibration scenario in accordance to the aircrew perception was the translational lift, in which significant $1 /$ rev and $4 /$ rev peaks were observed simultaneously at both frontback and vertical directions of the pilot as well as co-pilot helmet and shoulder locations.

Analysis results revealed that the vibration transmission from the floor to the pilot body was generally similar despite the differences in input vibration levels and spectral components. The frequency spectra of the vibration transmission path from the floor to the helmet at $120 \mathrm{kts}$ forward flight condition for the pilot and co-pilot in the vertical $\mathrm{Z}$ direction are shown in Figure 2.9 and Figure 2.10, respectively. The frequencies observed on the helicopter structure included $4 / \mathrm{rev}$ frequency at $21.6 \mathrm{~Hz}$ as the most dominant frequency followed by the $8 / \mathrm{rev}$ frequency at $43.2 \mathrm{~Hz}$ and $1 / \mathrm{rev}$ frequency at $5.4 \mathrm{~Hz}$. In contrast to the structural vibrations, the vibration measured on the pilot and co-pilot helmets and shoulders showed $1 / \mathrm{rev}$ at $5.4 \mathrm{~Hz}$ as the dominant frequency for the majority of flight conditions. It showed that the combination of the human body with the seat structure amplifies the low frequency at $1 / \mathrm{rev}$ while attenuating the high frequencies when transmitting the floor vibration to the aircrew helmets. Comparing the vibration spectra at the floor and aircrew body locations, it was found that the vibration peaks at the higher frequencies were reduced effectively through the isolation effect of the passive seat cushion as well as the aircrew body [98]. 


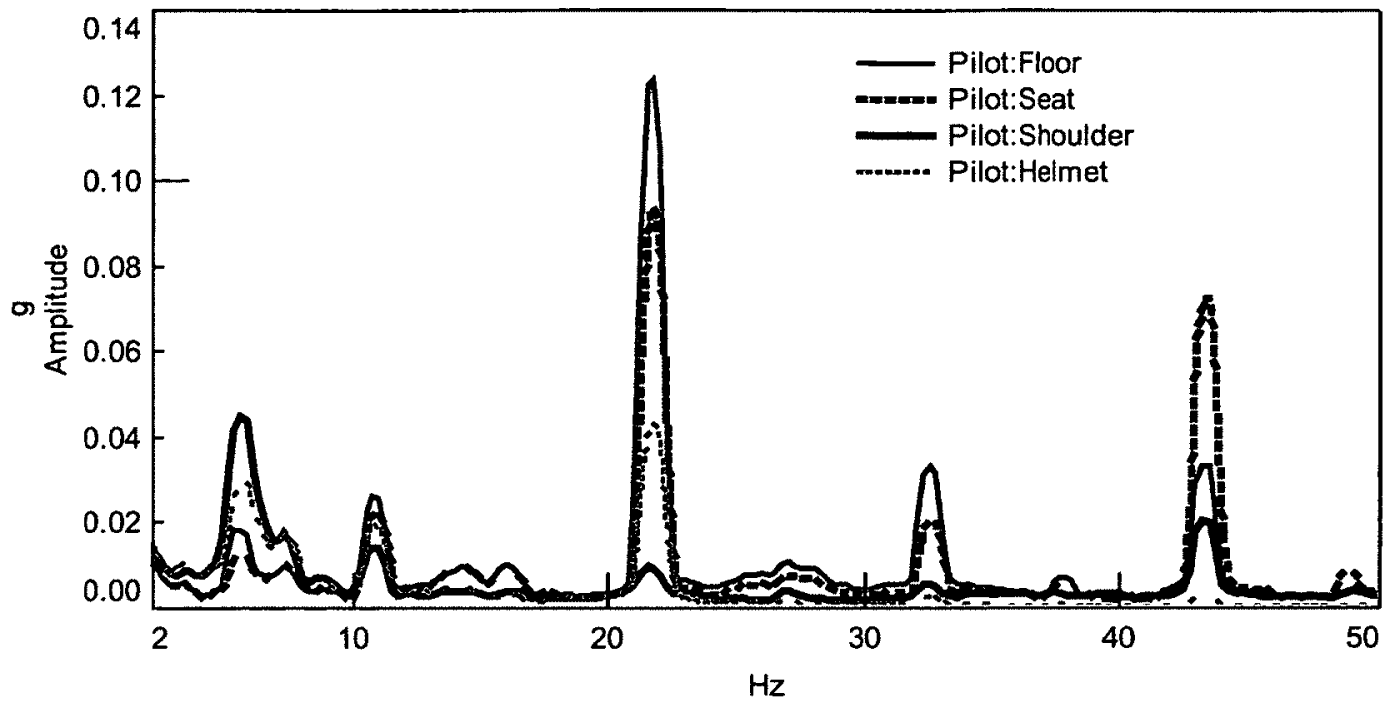

Figure 2.9: Transmission path spectra for the pilot in the Z-Direction for $120 \mathrm{kts}$

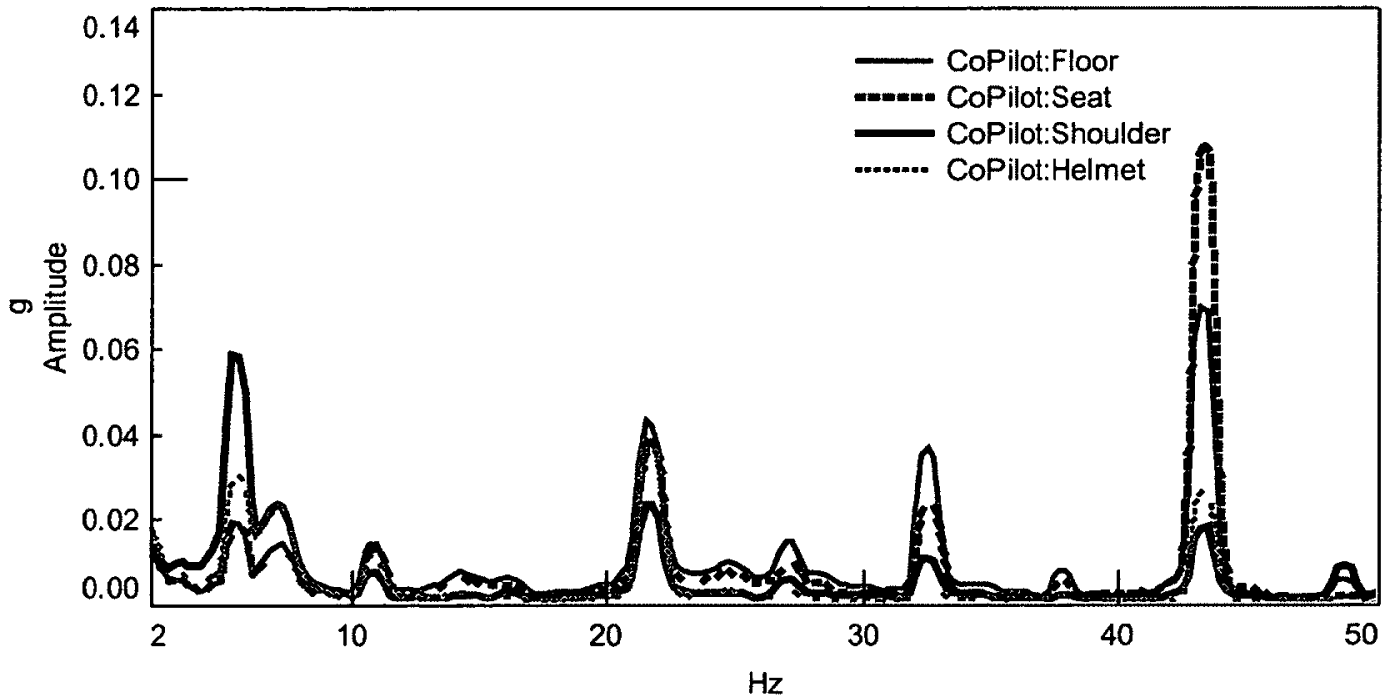

Figure 2.10: Transmission path spectra for the co-pilot in the Z-Direction for $120 \mathrm{kts}$

In particular, the $4 / \mathrm{rev}, 6 / \mathrm{rev}$ and $8 / \mathrm{rev}$ peaks observed at the floor location were reduced in the vibration spectra of pilot shoulder and helmet locations, while the amplitude at $4 / \mathrm{rev}$ was amplified. Due to the differences in aircrew weight, posture and other factors at the time of the measurement, the measured vibration amplitude also varied amongst the aircrew. 


\subsubsection{Transmissibility Analysis}

In order to evaluate vibration transmissibility characteristics through the seat structure, the transmissibility ratio, $\gamma$, was calculated for the frequency band of interest. The vibration transmissibility ratio for each target location and direction was calculated by using the autopower of the floor vibration spectrum in that particular direction as the reference as shown below. Frequencies with transmissibility ratios greater than one on the spectra indicate those frequencies that are amplified by the vibration transmission path. The transmissibility ratio is computed as the following ratio:

$$
\gamma=\frac{G_{x y}}{G_{y y}}
$$

where, $G_{x y}$ is the cross-power of floor and target accelerometer while $G_{y y}$ is the autopower of the floor vibration in the direction of the target accelerometer.

For example, the transmissibility ratio for the helmet location was calculated by using the autopower of the floor vibration spectrum in the $Z$ direction as the reference. The vibration transmissibility of the helmet location of the two aircrew members during the same flight is shown in Figure 2.11. Although pilot and co-pilot seats were equipped with the same type of seat cushion, the vibration transmissibility ratio varies slightly due to variations in the aircrew weight and posture. Despite the slight variation in frequency and amplitude, the similar transmissibility ratio was identified on both aircrew members. By examining the vibration transmissibility function between floor and the pilot helmet location in the frequency domain as shown in Figure 2.11, a head-helmet vibration mode at $6.2 \mathrm{~Hz}$ was identified as the front-back bending mode. In particular, the transmissibility spectra showed that the pilot and co-pilot helmets and shoulders significantly amplified the Z-direction vibration at the $1 /$ rev frequency of $5.4 \mathrm{~Hz}$. The transmissibility ratio analysis in the $\mathrm{X}$-direction showed a significant amplification peak at $7.3 \mathrm{~Hz}$. Similar transmissibility analysis in Y-direction did not show a significant amplification peak. Vibration transmissibility ratio analyses of flight test data indicates the existence of a structural resonance for the combined human body with the seat 
structure, at approximately $5.3 \mathrm{~Hz}$ and $7.3 \mathrm{~Hz}$ in the $\mathrm{Z}$ and $\mathrm{X}$ directions, respectively. The data indicated that the amplification of vibration in the $\mathrm{Z}$ direction was due to the coupling of this resonance mode with the $1 / \mathrm{rev}$ rotor harmonic peak at $5.4 \mathrm{~Hz}$. This vibration amplification may lead to potential injuries to the neck and spine of the pilot under long-term exposure of the helicopter vibratory environment. Additional weight on the flight helmet due to HUDs or NVGs can make this vibration situation much worse.

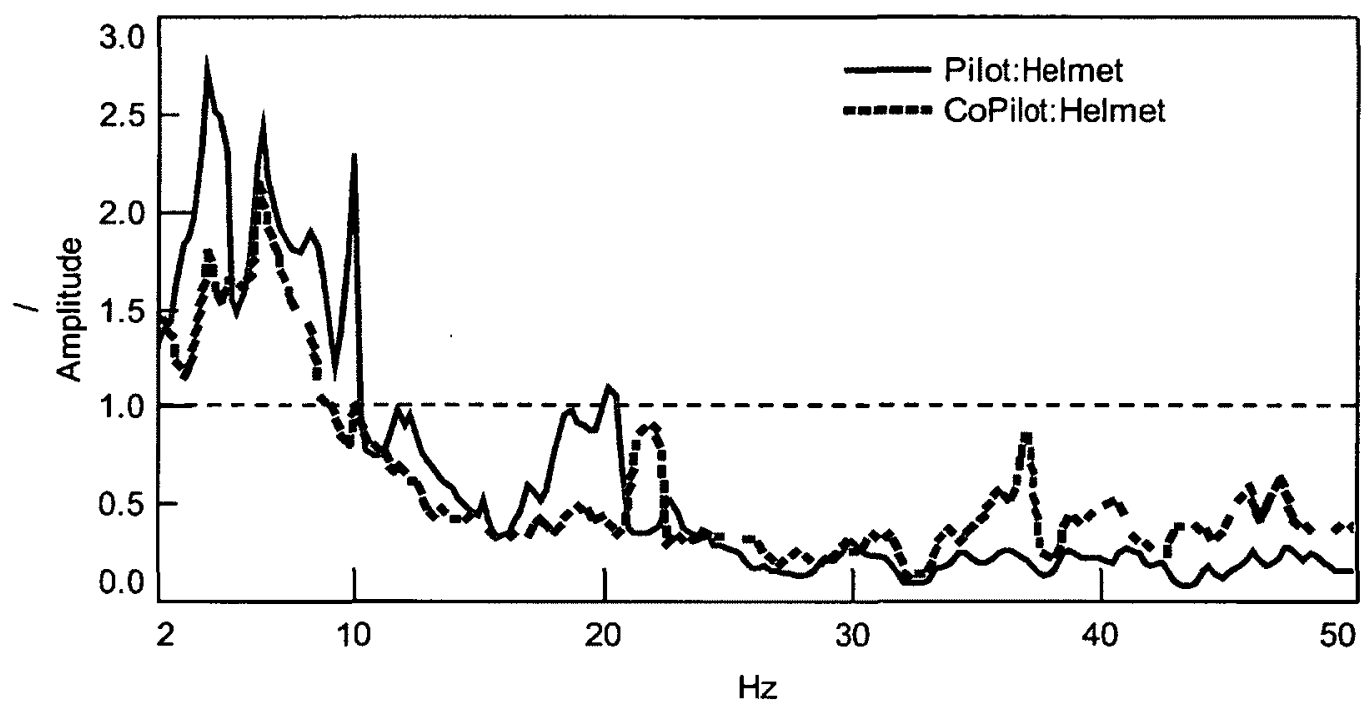

Figure 2.11: Vibration transmissibility for the helmet location in the Z-Direction

\subsection{Summary of the Flight Test}

A unique flight test performed using the NRC Bell-412 helicopter demonstrated that the helicopter aircrew is exposed to high levels of vibration during flight. The results showed that the aircrew vibration exposure levels varied substantially depending on operating conditions and aircrew configurations. Based on the commonly used ISO 2631 standard to assess the effect of the whole body vibration exposure, the data acquired during the flight test clearly concluded the necessity to reduce the level of vibration experienced by Bell-412 helicopter aircrew. For example, ISO 2631 comfort assessment and health guidance analysis indicated that most Bell-412 flight conditions were 
considered "uncomfortable" and "caution" while the $140 \mathrm{kts}$ flight condition was rated as "very uncomfortable" and "not safe," respectively.

Significant $1 / \mathrm{rev}$ and $4 / \mathrm{rev}$ vibration peaks were observed on the aircrew based on frequency domain spectral analysis. In contrast to the measured structural vibration, the transmissibility spectra showed that the pilot and co-pilot helmets and shoulders significantly amplified the Z-direction vibration at the $1 / \mathrm{rev}$ frequency of $5.4 \mathrm{~Hz}$. The flight test also verified that the currently used passive seat cushions do not provide satisfactory vibration isolation and furthermore long term-exposure at this level of vibration may be harmful to aircrew health.

Recognizing these challenges, it is necessary to develop innovative technologies to reduce the vibration levels experienced by the aircrew. Among them, the adaptive seat mount concept offers the adaptive capability to attenuate aircrew body vibration under various flight conditions and varied aircrew configurations at higher frequency regimes, while requiring minimum modification to the aircraft structure. In addition, the investigation of novel energy absorbing passive cushion material is required to improve the low frequency vibration attenuation through the aircrew seat. Therefore, development of a novel hybrid helicopter seat design integrated with both active and passive means is pursued to mitigate the vibration exposure of helicopter aircrew. 


\section{Chapter 3}

\section{Characterization of Seat System Dynamics for Simulation}

A biodynamic model of a seated aircrew was required to efficiently evaluate various novel seat designs through vibration simulations. This chapter briefly describes the comprehensive characterization tests performed, including the experimental modal analysis procedure, to extract dynamic parameters from the helicopter seat with a strapped mannequin that represented the aircrew. Modal test performed using a large electrodynamic shaker was also used to evaluate the effect of the aircrew weight and excitation level on the vibration response. The extracted modal parameters provided valuable information for the development of a lumped parameter based biodynamic model. This simplified model implemented using the SIMULINK software was an efficient simulation platform to evaluate effectively various seat design options to mitigate aircrew vibration in helicopters.

\subsection{Experimental Modal Analysis}

The experimental modal analysis methodology is one of the most common tools used to characterize the dynamics of mechanical systems because it produces very illustrative and useful results for structural design. Modal analysis results reveal critical dynamic properties of the structure required for vibration applications, namely, natural frequency, damping and the mode shapes. The extraction of modal properties of the helicopter seat 
structure was performed by measuring transfer functions from an experimental modal test. Although this section briefly discusses the theory associated with experimental modal analysis, it is not intended to provide a comprehensive background of this well established technology. In depth details of the modal analysis theory and implementation process has been published previously $[99,100,101]$.

\subsubsection{Brief Theoretical Background}

In experimental modal analysis, the dynamics of a structure is described in the frequency domain by a transfer function. A transfer function describes the response in one degreeof-freedom (DOF) due to a unity forcing in another DOF in the frequency domain. It is defined as:

$$
H_{i j}(\omega)=\frac{X_{i}(\omega)}{F_{j}(\omega)}
$$

where, $\omega$ is the frequency of excitation, $X_{i}(\omega)$ is the Fourier transform of the response $x_{i}(t)$ in DOF $i$, and $F_{j}(\omega)$ is the Fourier transform of a force $f_{j}(t)$ acting in DOF $j$. By measuring the vibration response $x_{i}$ and the applied force $f_{j}$, and performing the Fourier transformations, the transfer function $H_{i j}$ for a given DOF on the structure can be calculated using Eq. 3.1. This transfer function is one of $\mathrm{R} \times R$ transfer functions which can be measured on the seat structure with a $\mathrm{R}$ number of DOFs.

The system described above is an ideal one where the output is related directly to the input and it does not take noise into account. In experimental modal analysis, various estimators are used to account for the noise from the measured input and output signals in

calculating $H_{i j}$. The most commonly used one is the $H_{1}$ estimator, which assumes that there is no noise on the input and minimizes the noise on the output in a least squares sense. In this case the transfer function matrix $H_{1}$ is given by,

$$
H_{1}(\omega)=\frac{S_{F X}(\omega)}{S_{F F}(\omega)}
$$


where $S_{F X}(\omega)$ is the cross-spectrum of $x_{i}(t)$ and $f_{j}(t)$, while $S_{F F}(\omega)$ is the auto- spectrum of $f_{j}(t)$. Alternatively, the $\mathrm{H}_{2}$ estimator, which assumes that there is no noise on the output, can be used to obtain the transfer function matrix. It minimizes the noise on the input in a least squares sense and $\mathrm{H}_{2}$ is given by,

$$
H_{2}(\omega)=\frac{S_{X X}(\omega)}{S_{X F}(\omega)}
$$

where, $S_{X X}(\omega)$ is the auto- spectrum of $x_{i}(t)$.

The complete set of transfer functions is referred to as the transfer function matrix H. A transfer function matrix describes the dynamics between all DOFs of the structure and contains transfer functions between all combinations of DOF pairs. Modal parameters of the structure are extracted from measuring only one row or one column of the transfer matrix $\mathbf{H}$. A column of transfer functions is obtained by measuring the response in all DOFs, while the excitation point is fixed to one DOF. A row of $\mathbf{H}$ is obtained from measuring the response in one DOF while the point of excitation is moved between all DOFs. Any one of these measurements can be used to obtain all modal parameters of the structure.

The extraction of modal properties using experimentally obtained transfer functions is based on the formulation of the structural dynamics in the frequency domain. The natural frequencies of a structure are the eigenvalues and mode shapes are the eigenvectors of an eigenvalue problem. In order to verify this approach for modal analysis, consider the following linear equation of motion with external excitation:

$$
\mathbf{M} \ddot{\mathbf{x}}+\mathbf{C} \dot{\mathbf{x}}+\mathbf{S} \mathbf{x}=\mathbf{f}(t)
$$

where, where $x_{i}$ is the deflection in the DOF $i$, the vector $\mathbf{x}$ describes the discretized motion of the structure and the vector $\mathbf{f}$ is a forcing vector containing the external forces $f_{j}(t)$ which is acting in the DOFs $j=1,2,3, \ldots, R$ and where ()$\left.^{\prime}\right) \equiv d / d t$, and the matrices $\mathbf{M}, \mathbf{C}$ and $\mathbf{S}$ are the mass, damping and stiffness matrices. Proportional damping is 
assumed for the damping matrix $\mathrm{C}$. The Eq. 3.4 can be formed into an eigenvalue problem by assuming free vibration by assigning the right hand side to zero and inserting the solution $\mathbf{x}=v \mathrm{e}^{\lambda t}$ which yields:

$$
\left(\lambda^{2} \mathbf{M}+\lambda \mathbf{C}+\mathbf{S}\right) \mathbf{v}=\mathbf{0}
$$

The solution to the above Eq. 3.5 is the eigenvalues $\lambda_{k}$ and the corresponding eigenvectors $\mathbf{v}_{k}$ for $\mathrm{k}=1,2, . ., \mathrm{R}$. The eigenvalues of a damped structure are complex such that $\lambda_{k}=\sigma_{k}+i \omega_{k}$. As such, the natural frequency for the mode $k$ is given by:

$$
f_{k}=2 \pi \omega_{k}
$$

The eigenvector $v_{k}$ contain the modal amplitudes $v_{k, i}$ in all DOFs for mode number $k$. Once the eigenvectors $\mathbf{v}_{k}$ are known and the orthogonality of eigenvectors yields that the solution to Eq. 3.4 is written as follows:

$$
x(t)=\sum_{k=1}^{N} \mathbf{v}_{k} q_{k}(t)
$$

where, $q_{k}$ are the time-dependent generalized coordinates. Inserting Eq. 3.7 into Eq. 3.4 and multiplying by $\mathbf{v}_{k}^{T}$ from the left while using the orthogonality properties of eigenvectors detailed in reference [102], Eq. 3.4 reduces to

$$
\ddot{q}_{k}-2 \sigma_{k} \dot{q}_{k}+\left(\omega_{k}^{2}+\sigma_{k}^{2}\right) q_{k}=\mathbf{v}_{k}^{T} \mathbf{f}
$$

where $\mathrm{k}=1,2,3, \ldots, R$. The above Eq. 3.8 shows that the generalized coordinates $q_{k}$ are uncoupled in the equation of motion, indicating that the modes of the structure are uncoupled. These decoupled modes result in transfer functions that can be expressed as a sum of modal transfer functions. Performing Fourier transform expansions of Eq. 3.7 and 
Eq. 3.8, and combining them with the transfer function definition in Eq. 3.1 as detail in reference [102], the transfer function matrix is derived as

$$
\mathbf{H}(\omega)=\sum_{k=1}^{N} \mathbf{H}_{k}(\omega)=\sum_{k=1}^{N} \frac{\mathbf{v}_{k} \mathbf{v}_{k}^{T}}{\left(i \omega-\sigma_{k}-i \omega_{k}\right)\left(i \omega-\sigma_{k}+i \omega_{k}\right)}
$$

This Eq. 3.9 relates experimentally measurable transfer functions to the modal properties $\omega_{k}, \sigma_{k}$, and $\mathbf{v}_{k}$ for each mode $k$ to generate a modal transfer matrix $\mathbf{H}_{k}$ to the complete transfer function matrix consisting of all modes of the structure. Therefore, the sum of modal transfer functions $H_{k, i j}(\omega)$, which is an approximation of the measured transfer function, can be decomposed as

$$
H_{i j}(\omega) \approx \sum_{k=1}^{N} H_{k, i j}(\omega)=\frac{r_{k, i j}}{i \omega-p_{k}}+\frac{\bar{r}_{k, i j}}{i \omega-\bar{p}_{k}}
$$

where $p_{k}=\sigma_{k}+i \omega_{k}$ is the pole of the mode $k$ and $r_{k, i j}=v_{k, i} v_{k, j}$ is the residue of mode $k$ at DOF $i$ with reference to DOF $j$ while the bar denotes the complex conjugate. The pole of the mode is a complex quantity that combines the natural frequency and damping of the mode. The residue is also a complex quantity describing the product of two complex modal amplitudes. These modal properties are extracted from measured transfer functions by curve fitting functions derived from Eq. 3.10 with poles and residues as fitting parameters. A review of the experimental modal analysis process, including detailed discussions of common curve fitting parameter estimation methods, is found in the literature [102,103].

\subsubsection{Experimental Modal Test Setup}

An experimental modal analysis was conducted using a full-scale Bell-412 pilot seat to identify the major vibration modes of the seat that contribute to the aircrew vibrations. The vibration excitation to the seat structure was provided by the large shaker facility at 
NRC which is generally used for vibration and shock tests of aerospace components. The Unholtz-Dickie SA30-R16A electrodynamics shaker has the capability to generate $10,000 \mathrm{lbf}$ (peak) for sine, $10,000 \mathrm{lbf}(\mathrm{rms})$ for random and 20,000 lbf for shock tests within the frequency range from $5 \mathrm{~Hz}$ to $2000 \mathrm{~Hz}$. It is complete with a $1 \mathrm{~m} \mathrm{x} 1 \mathrm{~m}$ slip table and a $1 \mathrm{~m} \times 1 \mathrm{~m}$ head expander to test in 3 orthogonal directions independently. The input excitation to the shaker was generated using the LMS Test.Lab closed-loop Environmental Control software module. The shaker is capable of generating a number of vibration input profiles such as sine sweep, sine dwell, random, sine-on-random, random-on-random, as well as shock profiles such as half sine, terminal peak, triangular or shock response spectrum. Since the MIL-S-85510 standard stipulates that no vibration amplification shall occur within the frequency range of 4 to $50 \mathrm{~Hz}$, a low level random vibration spectrum within this frequency range at a level of $0.05 \mathrm{~g}-\mathrm{rms}$ was selected as the excitation source for the experimental modal test [9]. Low excitation was used to satisfy the.linear and time invariant system assumptions of the modal analysis process.

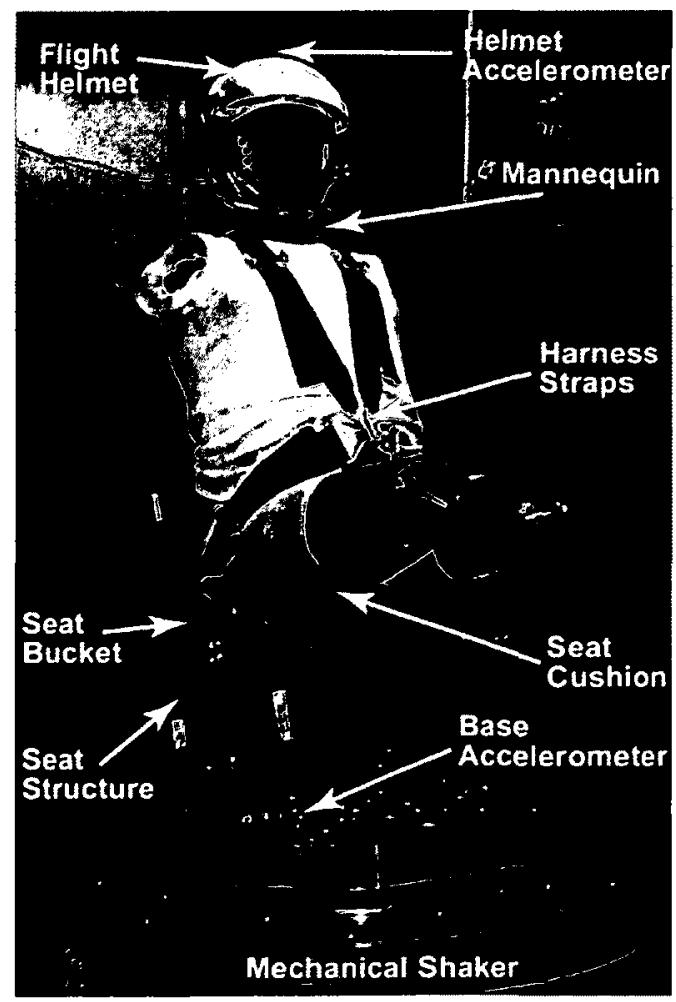

Figure 3.1: Seat modal test setup on the shaker with the mannequin 
The Bell-412 helicopter seat described in Section 2.2.3 was installed on the shaker in Z-direction excitation to generate helicopter floor vibration, as shown in Figure 3.1. The bottom of the seat frame was attached to the shaker armature through an interface plate. To evaluate the impact of a seated aircrew to the dynamics of helicopter seats, a Sierra Sam type mannequin that represented a $95^{\text {th }}$ percentile male was strapped to the seat in a normal seating posture using the restraint harness system. The limbs of the mannequin were removed to reduce noise in the measurement signal due to uncorrelated excitation. The mannequin was fitted with a typical flight helmet and the total weight of this modified mannequin was approximately $180 \mathrm{lbs}$. Special attention was given to the tension of the seat harness to minimize unwanted movements of the mannequin and to maintain consistency in the process of securing the mannequin by strapping to the seat. The mannequin provided a qualitative mass distribution of a seated pilot on the seat structure under vibration excitation. Use of this mannequin on the seat was necessary to extract relevant modal parameters of the Bell- 412 helicopter seat by representing the effect of the aircrew.

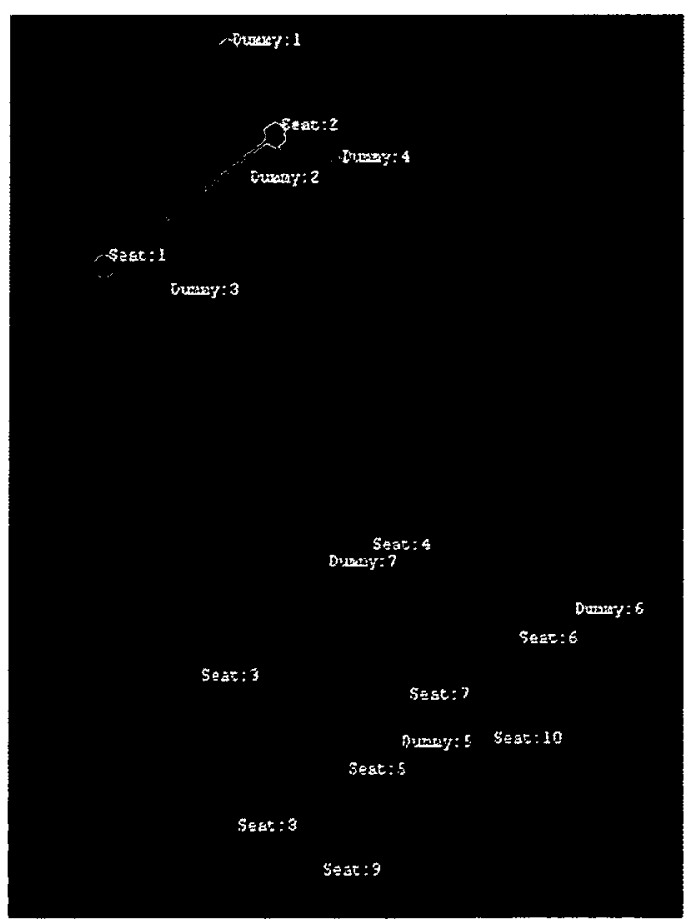

Figure 3.2: Geometry model for the experimental modal test 
The response of the seat structure was measured using 37 high sensitivity miniature accelerometers of the type PCB $357 \mathrm{C} 10$ and PCB 352C22. These low weight miniature accelerometers mitigated the effect of mass loading on the structure and their high sensitivity was ideal to obtain good signal-to-noise ratio in measurements even when the response amplitudes were relatively low. In order to provide a reasonably clear representation of the mode shapes of the seat structure, tri-axial accelerometers were attached to 6 locations on the seat frame and the seat bucket. In addition, tri-axial accelerometers were also installed on 6 locations on the mannequin to identify its movement. One accelerometer was also installed on the shaker interface plate directly underneath the helicopter seat and it was used as the shaker control sensor. The geometry model of the seat and mannequin used for the accelerometer layout is shown in Figure 3.2. All accelerometers were calibrated using the standard calibration procedure to ensure accuracy and reliability prior to installation.

The shaker excitation signal generation and data acquisition were performed using the LMS SCADAS 310 front-end hardware configured with one VDAC output card and four V12 input cards [104]. Simultaneous generation of the random excitation signal and the data acquisition of all channels were performed using the Random Control module of the LMS Test.Lab software [105].

\subsubsection{Experimental Modal Data Analysis}

The LMS Test.Lab Spectral Testing software package was used to calculate the FRF and Coherences from the recorded time domain data. The FRF was calculated as the ratio of the cross-spectrum between input and output and the power spectrum of the inputs based on $\mathrm{H} 1$ formulation, as defined in Eq. 3.2, which assumed noise-free input measurements. The signal from the control accelerometer placed on the vibrating base was used as the reference signal in calculating the FRFs of the response accelerometers. A frequency resolution of $0.1 \mathrm{~Hz}$ was selected for data analysis in order to distinguish any closely spaced modes. To improve the coherence and mitigate the measurement noise in the data, 30 linear averages were taken during each test run. Typical FRFs calculated for a sensor installed on the mannequin and a sensor placed on the seat are shown in Figure 
3.3. The calculated FRFs were assigned to the appropriate nodes of the geometry model in order to animate the identified mode shapes for proper visual display through animation.

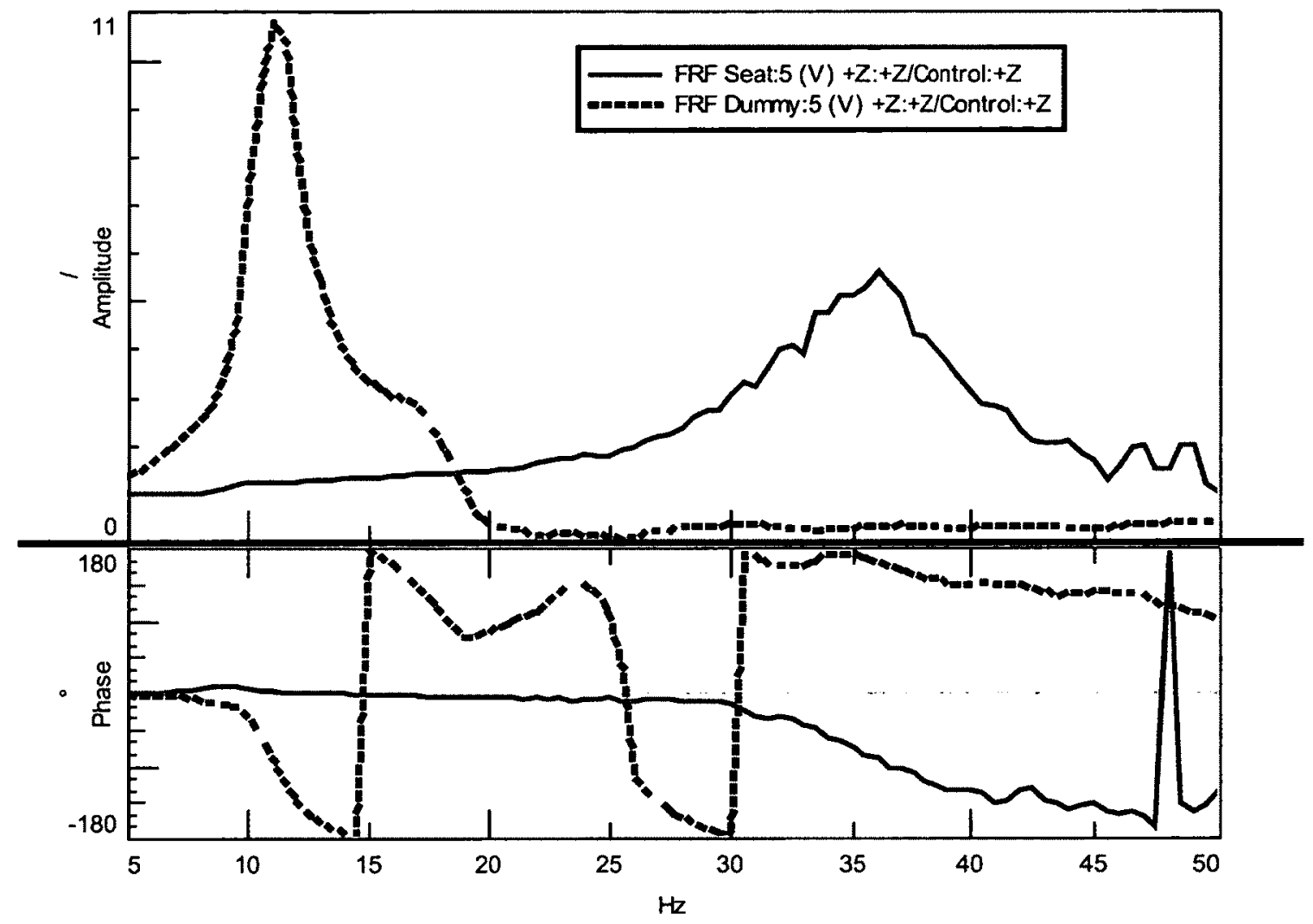

Figure 3.3: FRFs calculated from the shaker modal tests

The Modal Analysis module of LMS Test.Lab software was used to extract the modal parameters from the measured FRF data. The FRFs from each sensor, recorded separately during data acquisition, were combined and analyzed together for modal parameter estimation. The software used an advanced curve fitting algorithm known as PolyMAX to estimate frequency, damping and mode shape from the experimental test data. PolyMAX is an advanced polyreference least-squares complex frequency-domain (LSCF) algorithm that generates very clear stability diagrams for modal parameter estimation [106,107]. The PolyMAX method is a further evolution of the LSCF 
estimation used to find initial values for the iterative maximum likelihood method by incorporating complex frequency mapping to enhance the numerical characteristics [108, 109]. This advanced curve fitting method allowed curve fitting of a wide frequency band containing a high number of modes in a single analysis run. The algorithm was able to process all measurement DOFs within the complete frequency range of interest without much difficulty. The identification of the modal parameters using all FRFs was aided by the Multivariant Mode Indicator Function (MMIF) and the Sum of FRFs on the stability diagram generated by the PolyMAX curve fitter as shown in Figure 3.4. The MMIF is a frequency domain function that exhibit local minima at the natural frequencies of real normal modes to identify the resonance frequencies of the structure.

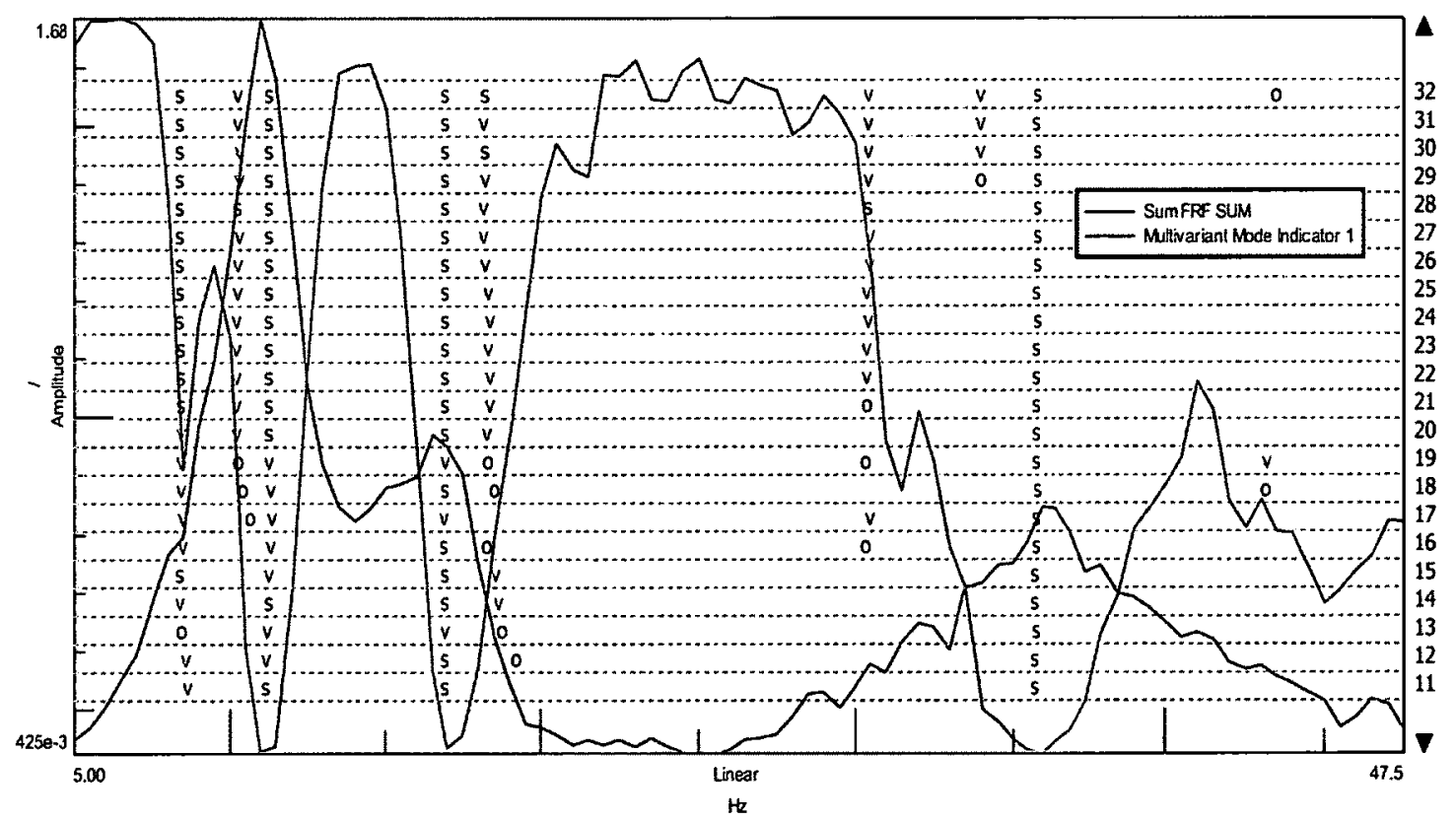

Figure 3.4: Stability diagram generated from the modal test

The stabilization diagram in Figure 3.4 is an important tool used to separate the physical system modes from mathematical poles [102]. In general, the stabilization diagram is generated by repeating the analysis for increasing model order. The vertical on the right shows the model order and the horizontal axis represents the damped natural frequency of the estimated modes. The symbol ' $s$ ' means that the variation over 
consecutive model orders of the damped natural frequency is smaller than $1 \%$ while the damping ratio varies less than $5 \%$. Once the modal parameters were extracted by selecting stable poles marked as " $\mathrm{s}$ " on the stability diagram, these parameters were used to synthesize the FRFs for each channel in order to compare with the FRFs generated from the experimental data [110]. Comparison of synthesized FRFs with the measured FRFs was important to determine the level of accuracy of the estimated modal parameters that represented the overall dynamics of the structure. In addition, extracted mode shapes were compared to each other using the Modal Assurance Criteria (MAC), which is an orthogonality check of the extracted modal parameters. When compared with all extracted mode shapes, the MAC matrix showed a value of near zero in the off-diagonal terms suggesting that the identified mode shapes were independent from each other.

\subsubsection{Modal Test Results}

The modal test was performed on the Bell-412 seat with and without the mannequin on the seat. The first test condition which included the Bell-412 seat structure only, identified two major vibration modes as shown in Figure 3.5. Since the seat pan with the cushions was attached to the vertical frame through a rectangular guide rail to allow for aircrew vertical position adjustment, the clearance between the rails introduced a bending mode to the seat bucket structure at $9.0 \mathrm{~Hz}$ as Mode 1. Mode 2 was as a major bending mode of the seat frame at $36.4 \mathrm{~Hz}$ due to the cantilever type motion of the seat frame.

The second modal test condition which included the strapped mannequin on the Bell-412 pilot seat structure identified six vibration modes as shown in Figure 3.6. Due to the addition of mannequin mass, the bending seat mode was decreased to $5.6 \mathrm{~Hz}$ (Mode 1). The mannequin head/helmet showed a localized bending mode in the fore-aft direction at $9.7 \mathrm{~Hz}$ (Mode 2). Due to the mannequin mass and seat cushion stiffness, a vertical mannequin mode was identified at $10.8 \mathrm{~Hz}$ (Mode 3). The cushion on the tilted seat back introduced two major vibration modes in the fore-aft direction: a mannequin body bending mode at $16.6 \mathrm{~Hz}$ (Mode 4) and a combined mannequin head/body movement mode at $19.8 \mathrm{~Hz}$ (Mode 5). Both of these two vibration modes incurred significant vibration levels to the mannequin head/helmet in the fore-aft direction. A 
second bending mode of the seat bucket/frame was also identified at $43.2 \mathrm{~Hz}$ due to the mass of the mannequin. Analysis of the vibration spectra revealed that the vibration modes at 5.6, 9.7, 10.8, 16.6 and $19.8 \mathrm{~Hz}$ contributed dominantly to the vibration levels at the mannequin helmet location. Suppression of these vibration modes could lead to relief of the back and neck injury problems for the helicopter aircrew.
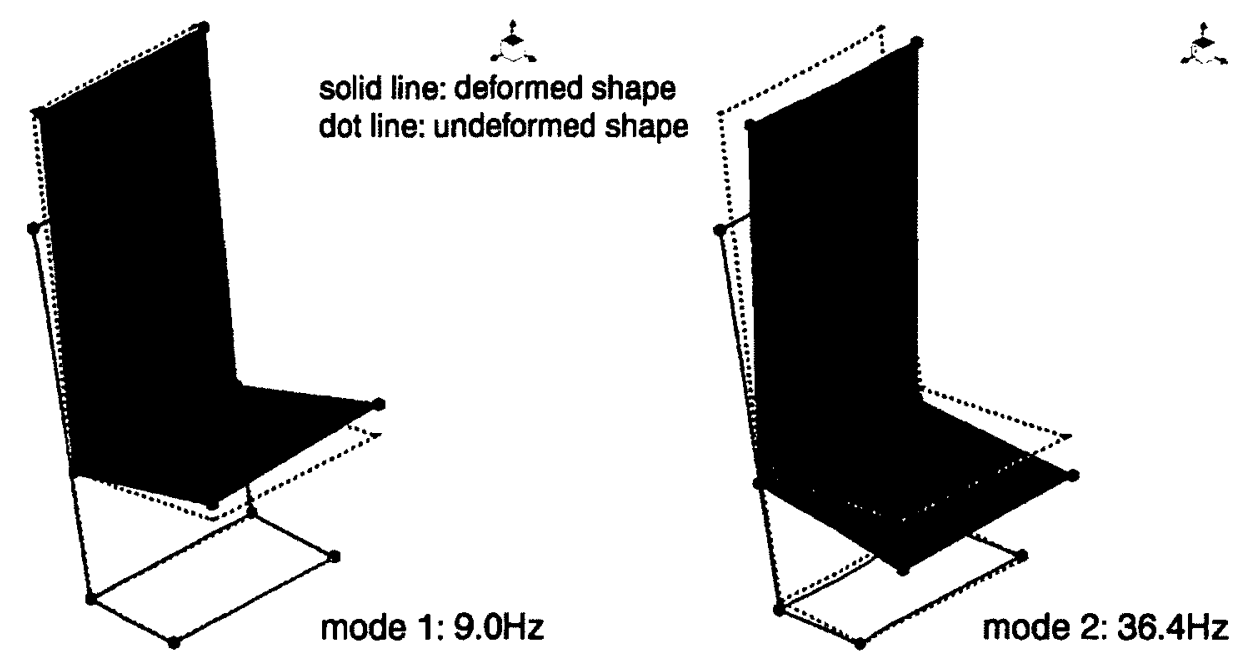

Figure 3.5: Seat modes without mannequin

Experimental modal parameter results verified that a few major vibration modes contributed to the excessive vibration levels of the aircrew body. The aircrew helmet and shoulder were exposed to large amplitude vibrations in both the vertical and fore-aft directions. It was determined that the dynamic coupling of the seat bending mode at 5.6 $\mathrm{Hz}$ (Mode 1) with the $1 / \mathrm{rev}$ rotor excitation may be the cause for vibration amplification in the low frequency regime. The critical modes that were identified from the experimental modal test were used to generate the biodynamic model of the seated aircrew to evaluate seat design options through vibration simulations. In addition, several simplified modal tests were performed using the shaker facility to investigate the variation in the modal frequencies due to the aircrew weight and the level of vibration input. 

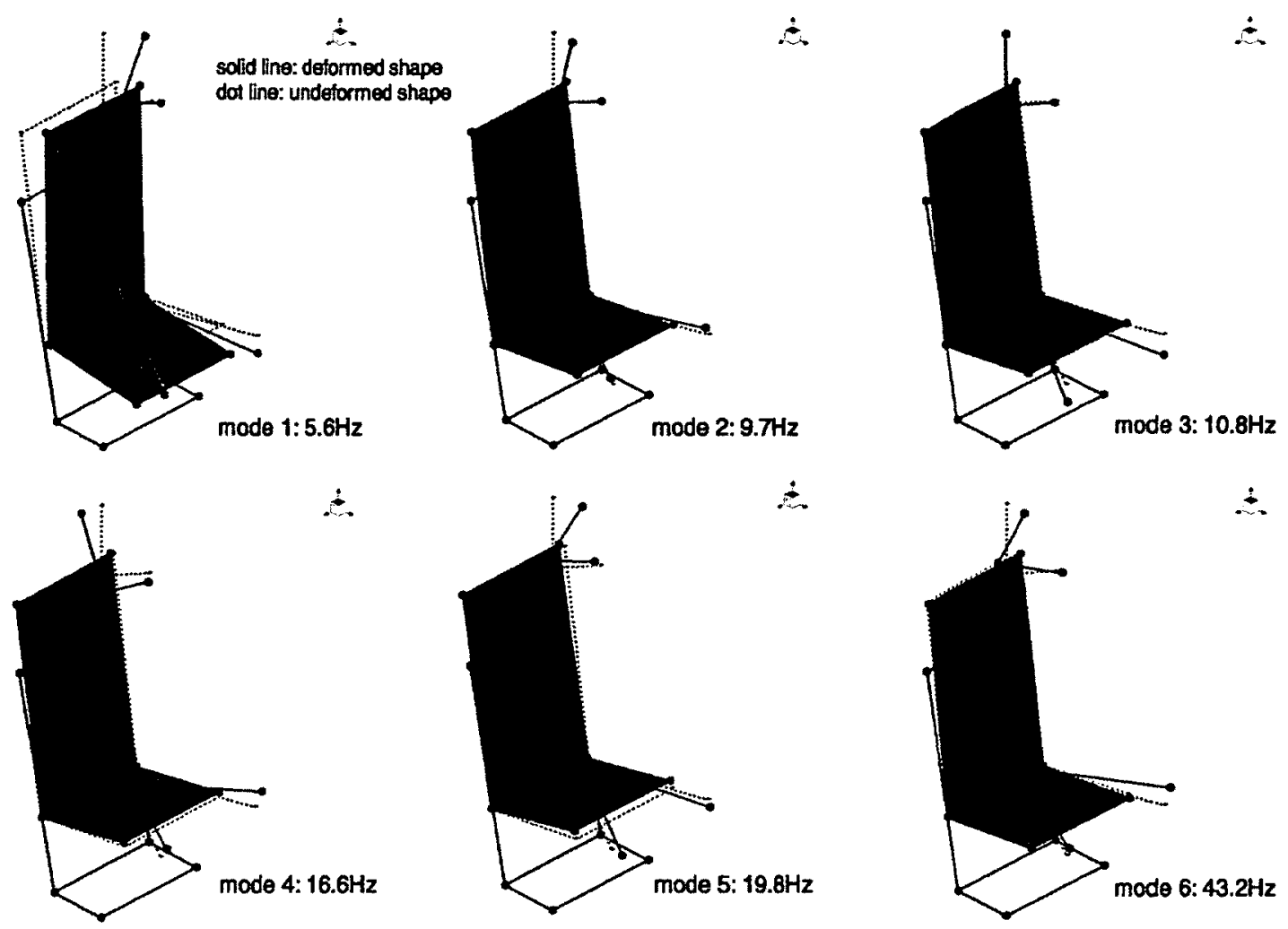

Figure 3.6: Seat modes with mannequin

\subsection{Identification of Factors that Affect Modal Parameters}

It was important to investigate the factors that affect variation in extracted modal parameters, primarily the modal frequencies. It was known that factors such as the aircrew weight, seated posture, restraint tension as well as the vibration level due to nonlinearity of cushion properties may affect modal frequencies. In this study, the variation in modal frequencies due to two factors, namely, the aircrew weight and the level of input vibration, were studied using the shaker tests performed on a seated mannequin.

\subsubsection{Variation in Aircrew on Modal Parameters}

It was important to identify the variation in the modal parameters for different aircrew body types. This task was accomplished by performing mechanical shaker tests on the 
Bell-412 helicopter seat by strapping three variations of Hybrid III mannequins, namely, $5^{\text {th }}$ percentile female weighing $108 \mathrm{lbs}, 50^{\text {th }}$ percentile male weighing $172 \mathrm{lbs}$ and $95^{\text {th }}$ percentile male weighing $223 \mathrm{lbs}$. These three mannequin configurations represented the range in population, particularly in weight and height. Unlike the Sierra Sam type mannequin, which only provided the mass distribution during the modal test, Hybrid III mannequins are full-scale anthropomorphic test devices that simulate the dimensions, weight proportions as well as the articulation of the human body. Therefore, these Hybrid III mannequins provide a more accurate representation of various body types of the helicopter aircrew with dynamics representative of many of the critical body parts. Mannequins were strapped to the full-scale helicopter seat, which was attached to the shaker head, using the existing safety belt restraint system. The seat height was adjusted according to the mannequin's height so that the feet rested on the shaker platform in order to provide a direct load transmission path from the shaker to the mannequin's feet. An accelerometer placed on the shaker table in the Z-direction was used as the reference and the response of the mannequin was measured using accelerometers installed inside the head, torso, chest cavities and the bottom seat cushion interface. The test setup with the three mannequins installed on the seat in the shaker facility is shown in Figure 3.7.

The shaker was excited using a random signal generated in the $3 \mathrm{~Hz}$ to $50 \mathrm{~Hz}$ band with an amplitude of $0.25 \mathrm{~g}$-rms to measure the FRFs. The FRFs calculated for most sensor locations showed that the resonance frequencies as well as the transmissibility amplitudes varied as the mannequin changed from $5^{\text {th }}$ percentile female to $50^{\text {th }}$ percentile male to $95^{\text {th }}$ percentile male. For example, the variation in the FRFs measured by the accelerometer located on the mannequin head in Z-direction is shown in Figure 3.8. The resultant resonance frequency and transmissibility amplitudes for measured locations of each mannequin are tabulated in Table 3.1. The resonance frequency and amplitude variation measured on the seat cushion in the Z-direction illustrate the change in modal parameters as the mannequin weight changed from $108 \mathrm{lbs}$ to $172 \mathrm{lbs}$ to $223 \mathrm{lbs}$. 


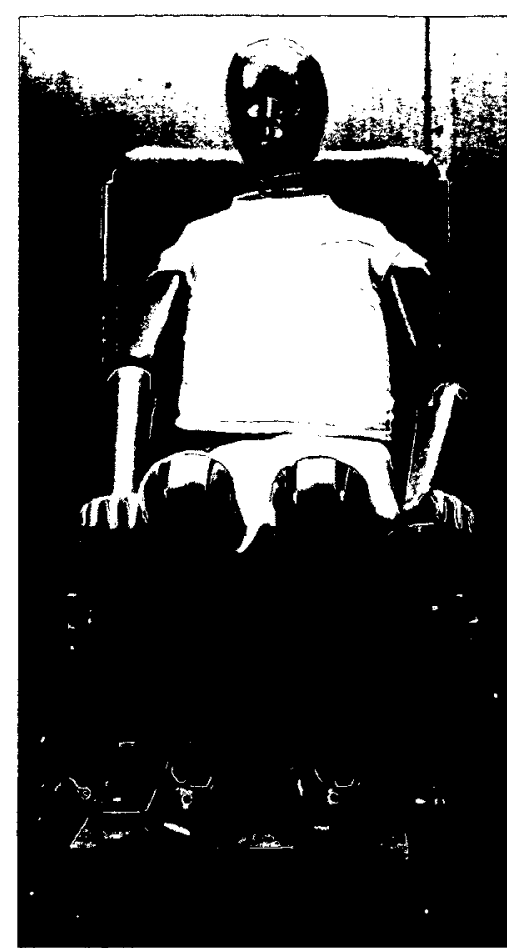

$5^{\text {th }}$ Percentile female

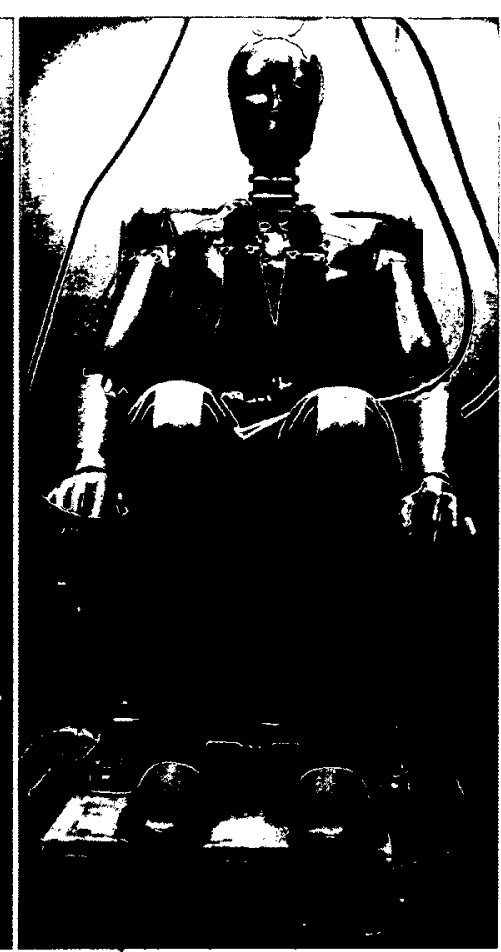

$50^{\text {th }}$ Percentile Male

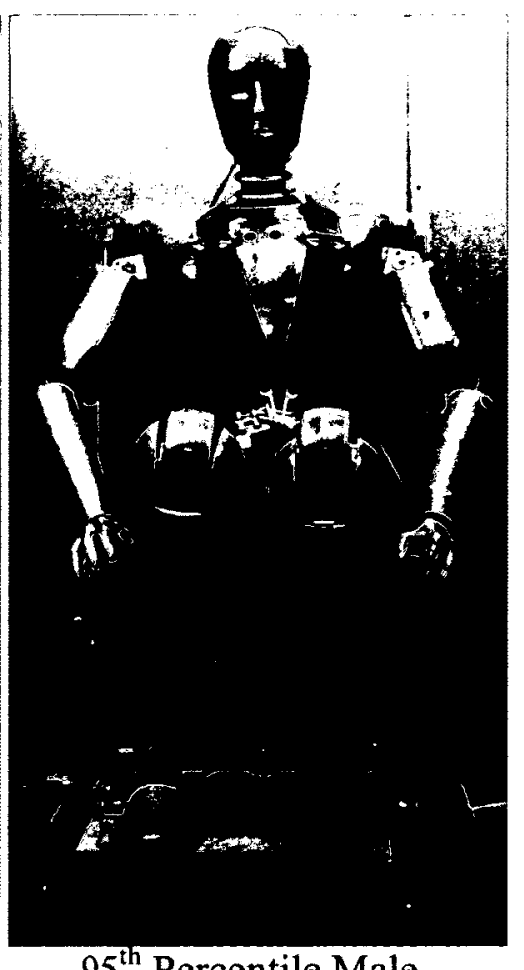

$95^{\text {th }}$ Percentile Male

Figure 3.7: Hybrid III mannequins installed on the shaker

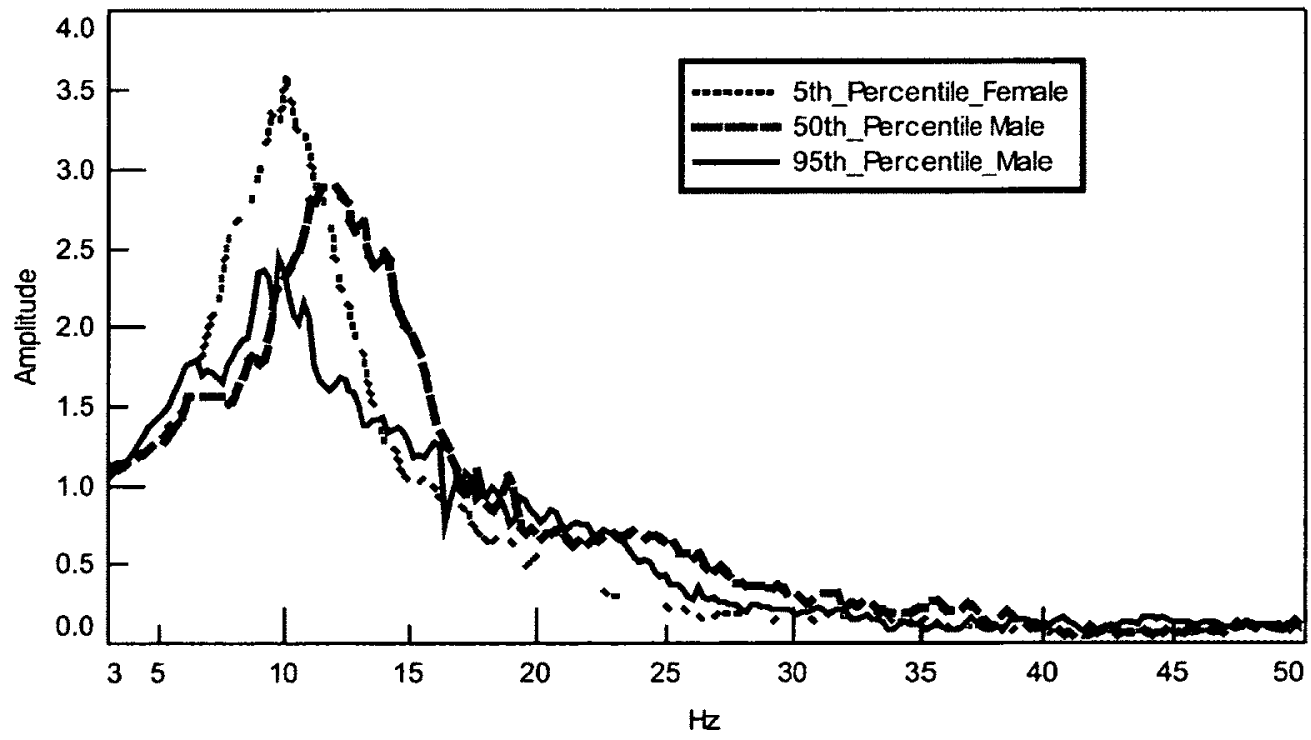

Figure 3.8: FRFs measured from mannequin head accelerometer in Z-direction 
Table 3.1: Variation in vibration resonance characteristics for each mannequin

\begin{tabular}{|l|c|c|c|c|c|c|}
\hline & \multicolumn{3}{|c|}{ Resonance Frequency $\mathbf{( H z )}$} & \multicolumn{3}{c|}{ Transmissibility Amplitude } \\
\hline Mannequin Type & $\mathbf{5}^{\text {th }} \mathbf{F e m a l e}$ & $\mathbf{5 0}^{\text {th }} \mathbf{M a l e}$ & $\mathbf{9 5}^{\text {th }} \mathbf{M a l e}$ & $\mathbf{5}^{\text {th }}$ Female & $\mathbf{5 0}^{\text {th }} \mathbf{M a l e}$ & $\mathbf{9 5}^{\text {th }}$ Male \\
\hline Head-Z direction & 10.0 & 11.8 & 9.8 & 3.5 & 2.9 & 2.3 \\
\hline Head-X direction & 8.2 & 7.2 & 7.0 & 1.0 & 1.2 & 2.6 \\
\hline Chest-Z direction & 10.0 & 11.8 & 9.8 & 3.2 & 3.1 & 2.9 \\
\hline Chest-X direction & 11.2 & 23.6 & 20.4 & $\mathbf{0 . 7}$ & 0.5 & 0.5 \\
\hline Torso-Z direction & 10.0 & 11.8 & 9.8 & 3.2 & 2.9 & 3.2 \\
\hline Torso-X direction & 7.0 & 13.8 & 9.2 & 0.8 & 1.1 & 0.9 \\
\hline Cushion-Z direction & 12.8 & 11.6 & 10.2 & 2.5 & 2.4 & 2.3 \\
\hline Cushion-X direction & 7.0 & 12.8 & 21.2 & 0.8 & 0.7 & 1.1 \\
\hline
\end{tabular}

\subsubsection{Variation in Vibration Input Level on Modal Frequencies}

The shaker tests were also used to evaluate the effect of vibration input levels on the modal frequency variation on the aircrew excitation. This was achieved by vibrating the $50^{\text {th }}$ percentile Hybrid III mannequin strapped to the Bell-412 seat at several shaker excitation levels. The four random vibration levels, namely, $0.5 \mathrm{~g}$-rms $(0.0 \mathrm{~dB}), 0.36 \mathrm{~g}-$ $\mathrm{rms}(-3.0 \mathrm{~dB}), 0.25 \mathrm{~g}-\mathrm{rms}(-6.0 \mathrm{~dB})$ and $0.18 \mathrm{~g}$-rms $(-9.0 \mathrm{~dB})$, were generated using the shaker test facility for this experimental investigation. The FRFs calculated for selected sensor locations showed that the resonance frequencies as well as the transmissibility amplitudes varied as the input vibration level was varied. For example, the variation in the FRFs measured by the accelerometer located on the bottom cushion interface in Zdirection is shown in Figure 3.9.

The test results show that the resonance frequency as well as the transmissibility amplitude measured on the seat cushion decreased with the increase of input vibration levels. Variation in transmissibility due to the level of input excitation is a classical sign of a nonlinear material behavior. This observation demonstrated that the helicopter seat cushion impedance properties were highly nonlinear. The resultant resonance frequency and transmissibility amplitudes for the measured locations for each input vibration level are tabulated in Table 3.2 . 


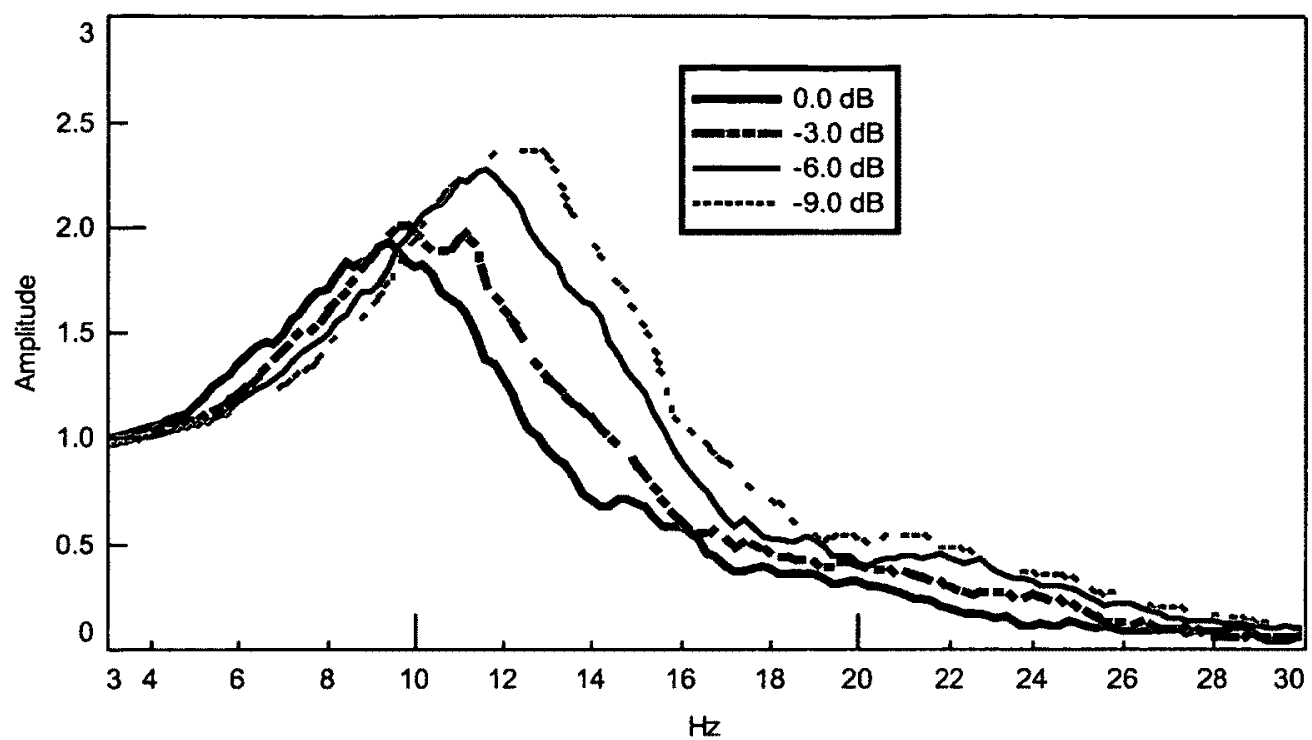

Figure 3.9: FRFs measured on the cushion interface in Z-direction

Table 3.2: Variation in vibration resonance characteristics with input levels

\begin{tabular}{|c|c|c|c|c|c|c|c|c|}
\hline & \multicolumn{4}{|c|}{ Resonance Frequency (Hz) } & \multicolumn{3}{c|}{ Transmissibility Amplitude } \\
\hline Input Vibration Level & $\mathbf{0 . 0 ~ d B}$ & $\mathbf{- 3 . 0} \mathbf{~ d B}$ & $\mathbf{- 6 . 0 ~ d B}$ & $\mathbf{- 9 . 0 ~ d B}$ & $\mathbf{0 . 0 ~ d B}$ & $\mathbf{- 3 . 0} \mathbf{~ d B}$ & $\mathbf{- 6 . 0 ~ d B}$ & $\mathbf{- 9 . 0 ~ d B}$ \\
\hline Head-Z direction & 9.4 & 11.2 & 11.8 & 13.0 & 2.5 & 2.7 & 2.9 & 3.1 \\
\hline Head-X direction & 6.4 & 7.2 & 7.5 & 7.8 & 1.0 & 1.1 & 1.2 & 1.3 \\
\hline Chest-Z direction & 9.4 & 11.2 & 11.8 & 13.0 & 2.6 & 2.8 & 3.1 & 3.3 \\
\hline Chest-X direction & 19.8 & 22.4 & 23.6 & 23.8 & 0.4 & 0.4 & 0.5 & 0.6 \\
\hline Torso-Z direction & 9.4 & 11.2 & 11.8 & 13.0 & 2.5 & 2.7 & 2.9 & 3.1 \\
\hline Torso-X direction & 13.2 & 13.4 & 13.8 & 13.2 & 0.9 & 1.0 & 1.1 & 1.2 \\
\hline Cushion-Z direction & 9.4 & 9.8 & 11.6 & 12.0 & 1.9 & 2.0 & 2.3 & 2.4 \\
\hline Cushion-X direction & 25.4 & 26.6 & 26.0 & 25.8 & 0.5 & 0.6 & 0.6 & 0.5 \\
\hline
\end{tabular}

\subsection{Development of a Biodynamic Model}

Based on the results of the modal tests of the helicopter seat and dynamics of the human body, a biodynamic model was developed to efficiently evaluate novel seat design options through vibration simulations. The development of biodynamic models to simulate responses of a seated human body has been the topic of interest over the years and a number of mathematical models have been previously established $[111,112]$. In general, these models fall into three broad techniques, namely, lumped parameter (LP), 
finite element (FE), and multi-body (MB) models. However, the LP model is one of the most popular analytical methods for the study of biodynamic responses of a seated body. This type of single dimensional (1-D) model simplifies the dynamics of the seat structure as well as the human for efficient implementation [113]. In contrast, the FE and MB models generally developed in three dimensions (3-D) are very complex for this application and require knowledge of a number of mechanical properties of the seat and the human which have been difficult to measure accurately [114]. The flight tests data measured on the aircrew was used to validate the developed lumped parameter model in this study. The validated model was used to evaluate the effectiveness of various options considered for the adaptive seat mount design and evaluate novel energy absorbing cushion materials to mitigate aircrew exposure to vibration.

\subsubsection{Lumped Parameter Model}

The human body in a seated posture along with the seat structure were modeled in the vertical direction as a mechanical system composed of several rigid bodies interconnected by springs and dampers. In this study, a four degree-of-freedom human body model previously developed by Wan and Schimmel for vertical random vibration simulation was used to represent the helicopter aircrew [111]. This model treats the upper body of the seated aircrew as four single degree-of-freedom (SDOF) masses interconnected by springs and dampers [115]. The mass $\mathrm{mp}_{1}$ represent the lower torso, $m p_{2}$ the viscera, $\mathrm{mp}_{3}$ the upper torso and $\mathrm{mp}_{4}$ represent the head and neck. In addition, the seat frame structure and the seat cushion were modeled as separate 1-DOF systems represented as $m_{F}$ and $m_{C}$, respectively [116]. Although the cushion and the seat frame are modeled together as a single mass-spring system in general, these components were considered separately for this investigation to study the effect of integrating novel cushion materials to the helicopter seat [93]. It is known that passive seat cushion materials have been used successfully to mitigate low frequency vibration transmission through seat structures [116]. The lumped parameter model shown in Figure 3.10 identifies the position of each corresponding lumped mass as $z_{F}, z_{C}$ and $z_{p}$ to $z_{4}$. 
Table 3.3: Mass, stiffness and damping parameters used in the model

\begin{tabular}{|c|c|c|}
\hline Mass (kg) & Damping (N-s/m) & Stiffness (N/m) \\
\hline $\mathrm{m}_{\mathrm{F}}=15$ & $\mathrm{c}_{\mathrm{F}}=41$ & $\mathrm{k}_{\mathrm{F}}=148299$ \\
\hline $\mathrm{m}_{\mathrm{C}}=1$ & $\mathrm{c}_{\mathrm{C}}=390$ & $\mathrm{k}_{\mathrm{C}}=47700$ \\
\hline $\mathrm{mp}_{1}=48.4$ & $\mathrm{cp}_{1}=2475$ & $\mathrm{kp}_{1}=49340$ \\
\hline $\mathrm{mp}_{2}=7.4$ & $\mathrm{cp}_{2}=330$ & $\mathrm{kp}_{2}=20000$ \\
\hline $\mathrm{mp}_{3}=20.2$ & $\mathrm{cp}_{3}=200$ & $\mathrm{kp}_{3}=10000$ \\
\hline $\mathrm{mp}_{4}=5.6$ & $\mathrm{cp}_{4}=250$ & $\mathrm{kp}_{4}=134400$ \\
\hline & $\mathrm{cp}_{13}=909.1$ & $\mathrm{kp}_{13}=192000$ \\
\hline
\end{tabular}

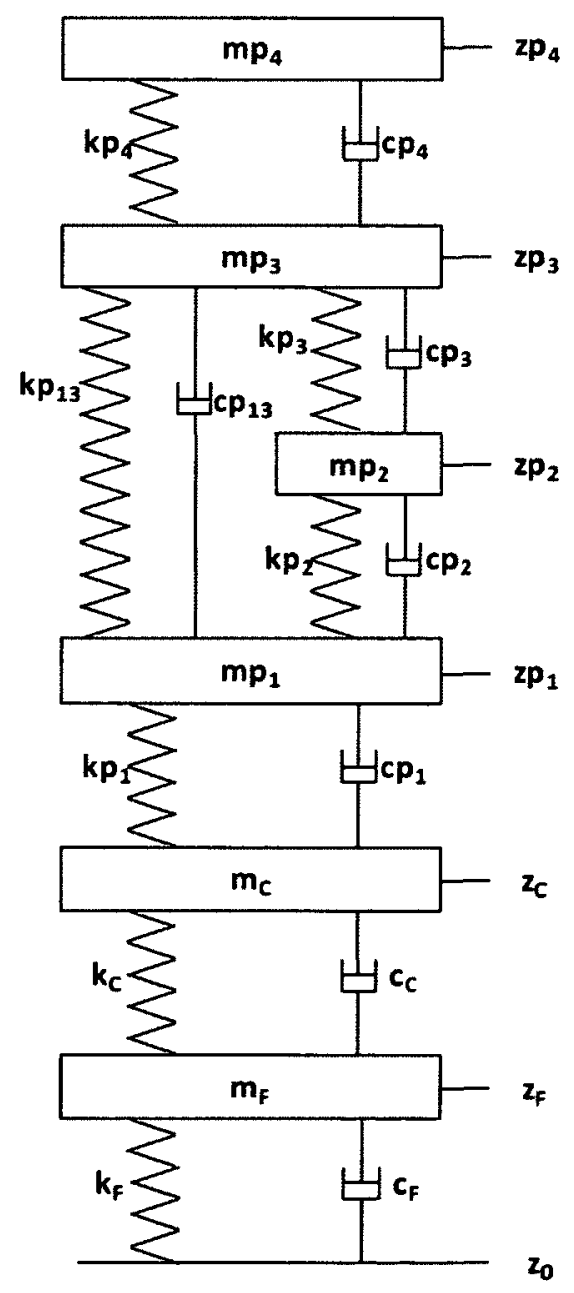

Figure 3.10: Lumped parameter model of the seated aircrew 
The floor displacement identified as $z_{0}$ is the input vibration to the lumped parameter model. The vibration measured in the base of the seat frame during the flight test is used as the vibration input in the dynamic simulation. Moreover, the measured mass of the pilot in standing and seated positions were used to derive the lumped masses associated with the human body. The mass of the lower torso was assumed to be $59 \%$ of the seated mass, the viscera was $9 \%$, the upper torso was $25 \%$ and the head and neck were $7 \%$. An additional $1 \mathrm{~kg}$ was added to the head mass to represent mass of the flight helmet worn by the pilot. However, damping and stiffness values were not changed from the original values identified in the Wan and Schimmel study [111].

The stiffness $\left(\mathrm{k}_{\mathrm{F}}\right)$ and damping $\left(\mathrm{c}_{\mathrm{F}}\right)$ values of the seat frame were extracted using the results of the experimental modal test performed on the seat with and without the mannequin. Additional shaker tests were conducted on the bare cushion material in order to extract approximate damping $\left(\mathrm{c}_{\mathrm{C}}\right)$ and stiffness $\left(\mathrm{k}_{\mathrm{C}}\right)$ values for the properties associated with only the bottom cushion. All parameters used in the lumped parameter model including mass, stiffness and damping are tabulated in Table 3.3.

\subsubsection{Formulation of Equations of Motion}

The equations of motion describing the dynamic behavior of the system are derived for the model using Newton second law of motion as follows:

$$
\begin{aligned}
& m_{F} \ddot{z}_{F}=k_{F} z_{0}+c_{F} \dot{z}_{0}-\left(k_{F}+k_{C}\right) z_{F}+k_{C} z_{C}-\left(c_{F}+c_{C}\right) \dot{z}_{F}+c_{C} \dot{z}_{C} \\
& m_{C} \ddot{z}_{C}=k_{C} z_{F}-\left(k p_{1}+k_{C}\right) z_{C}+k p_{1} z p_{1}+c_{C} \dot{z}_{F}-\left(c p_{1}+c_{C}\right) \dot{z}_{C}+c p_{1} \dot{z} \\
& m p_{1} \ddot{z} p_{1}=k p_{1} z_{C}-\left(k p_{1}+k p_{2}+k p_{2 b}\right) z p_{1}+k p_{2} z p_{2}+k p_{2 b} z p_{3}+c p_{1} \dot{z}_{C} \\
& -\left(c p_{1}+c p_{2}+c p_{2 b}\right) \dot{z} p_{1}+c p_{2} \dot{z} p_{2}+c p_{2} \dot{z} p_{2} \\
& m p_{2} \ddot{z} p_{2}=k p_{2} z p_{1}-\left(k p_{2}+k p_{3}\right) z p_{2}+k p_{3} z p_{3}+c p_{2} \dot{z} p_{1}-\left(c p_{2}+c p_{3}\right) \dot{z} p_{2}+c p_{3} \dot{z} p_{3} \\
& m p_{3} \ddot{z} p_{3}=k p_{2 b} z p_{1}+k p_{3} z p_{2}-\left(k p_{2 b}+k p_{3}+k p_{4}\right) z p_{3}+k p_{4} z p_{4}+c p_{2 b} \dot{z} p_{1} \\
& +c p_{3} \dot{z} p_{2}-\left(c p_{2 b}+c p_{3}+c p_{4}\right) \dot{z} p_{3}+c p_{4} \dot{z} p_{4} \\
& m p_{4} \ddot{z} p_{4}=k p_{4} z p_{3}-k p_{4} z p_{4}+c p_{4} \dot{z} p_{3}-c p_{4} \dot{z} p_{4}
\end{aligned}
$$


The system of equations represented in Eq. 3.9 depicts the equations of motion for all lumped masses of the model and this system of equations was transformed into the State Space model in the form:

$$
\begin{aligned}
& \dot{x}=A x+B u \\
& y=C x+D u
\end{aligned}
$$

by assigning variables shown below;

$$
\begin{aligned}
& x_{1}=z_{F} ; x_{2}=z_{C} ; x_{3}=z p_{1} ; x_{4}=z p_{2} ; x_{5}=z p_{3} ; x_{6}=z p_{4} \\
& x_{7}=\dot{z}_{F} ; x_{8}=\dot{z}_{C} ; x_{9}=\dot{z} p_{1} ; x_{10}=\dot{z} p_{2} ; x_{11}=\dot{z} p_{3} ; x_{12}=\dot{z} p_{4}
\end{aligned}
$$

The transformation of the variables produced A, B, C and D matrices of the State Space model and these matrices are presented in Appendix B. The matrix C was constructed to observe the displacement of desired component of the biodynamic model, primarily the vibration of the aircrew head.

\subsubsection{Performance of Dynamic Simulation}

The SIMULINK tool, provided as an extension in the MATLAB software, was used to perform the dynamic simulation using the developed lumped parameter model. It provides a graphical environment for the construction of a block diagram representation of a dynamic system for analysis. It contains a number of libraries which allow accurate modeling of a variety of continuous and discrete systems for dynamic simulation.

The floor vibration measured from the flight test in the vertical Z-direction was directly applied to the model as the excitation input. The State Space formulation shown in Figure 3.11 was used to implement matrices for $\mathrm{A}, \mathrm{B}, \mathrm{C}$ and D in SIMULINK environment. The vector $\mathbf{u}$ denotes the input, namely, position and velocity of the helicopter floor and the vector $\mathbf{y}$ denotes the output, namely, positions of the aircrew helmet. 


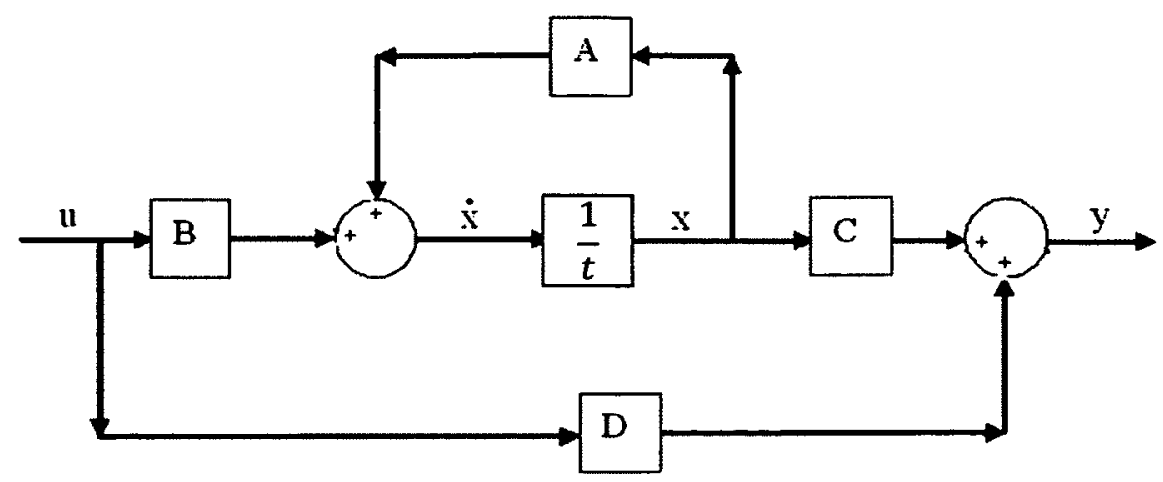

Figure 3.11: State Space formulation

\subsubsection{Dynamic Simulation Results}

The validation of the SIMULINK model was performed using sine sweep excitation as in input to the developed biodynamic seat model. In addition, multiple sine tones were used as the input to simulate the helicopter rotor excitation without the contribution of the random excitation. Finally, the floor vibration data recorded during the flight test, that included the sine tones of the rotor with random excitation, was used to represent the realistic vibration input to the seat structure. The dynamic simulation results of the model was compared to FRFs of several sensor signals, such as the seat frame, cushion interface and helmet of the pilot recorded during several flight conditions. A comparison of simulation results and flight test data of the FRF of the aircrew helmet is shown in Figure 3.12. The FRF comparisons showed that the simulation results does not fit perfectly due to its simplicity but represents the dominant characteristics of the system very well. This was evident by analyzing the time domain response of the pilot helmet acceleration simulated with the helicopter floor vibration. For example, a comparison of the time domain simulation results for the $120 \mathrm{kts}$ flight condition, shown in Figure 3.13, clearly falls within the range of accelerations measured during the flight test. Although this simplified lumped parameter model was not extremely accurate, the fidelity of the model was sufficient to perform parametric studies to evaluate various design options for the helicopter seat to mitigate vibration such as novel energy absorbing cushion materials and adaptive mount systems with active control technology. 


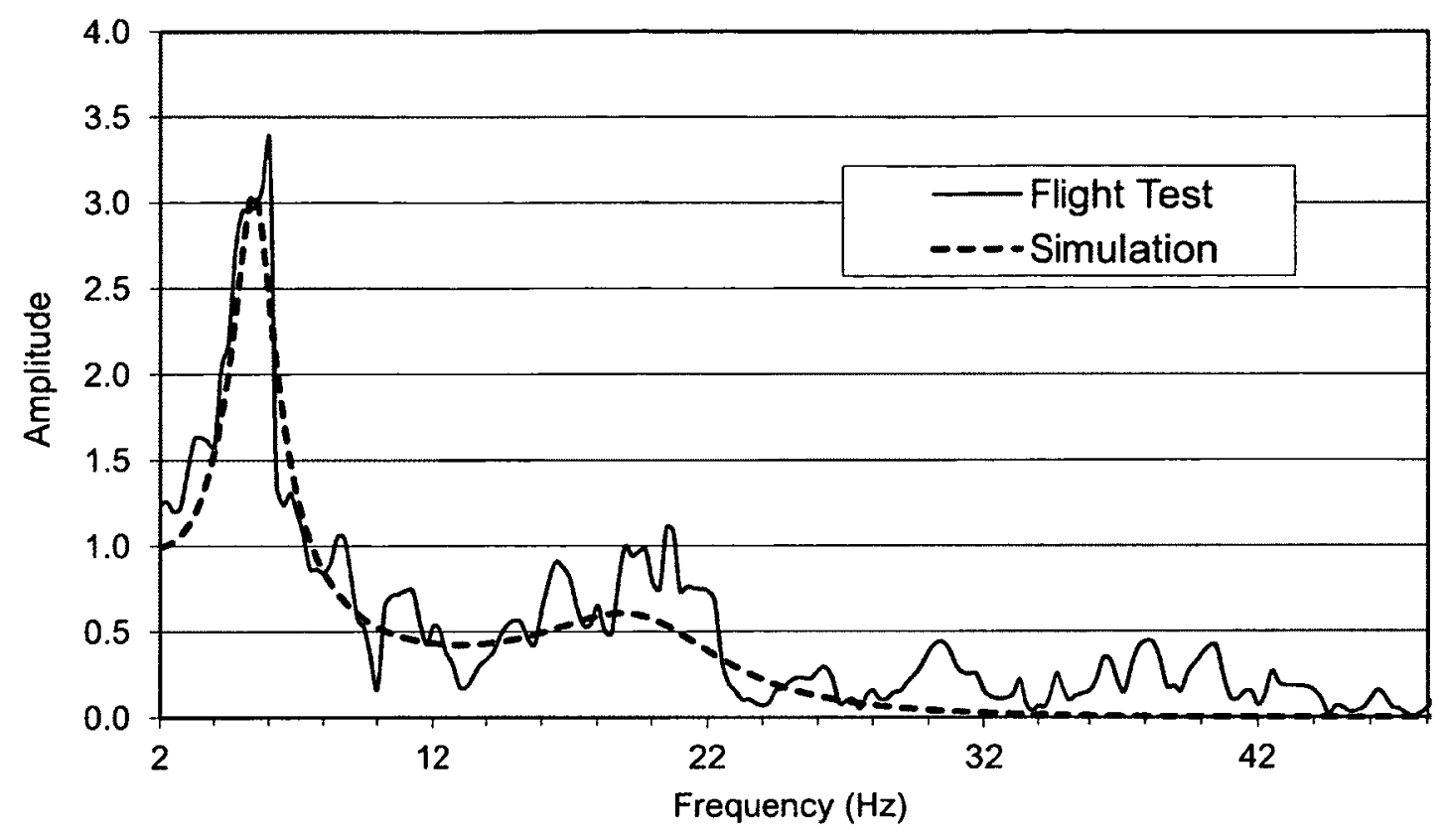

Figure 3.12: Helmet FRF comparison for simulation and flight test

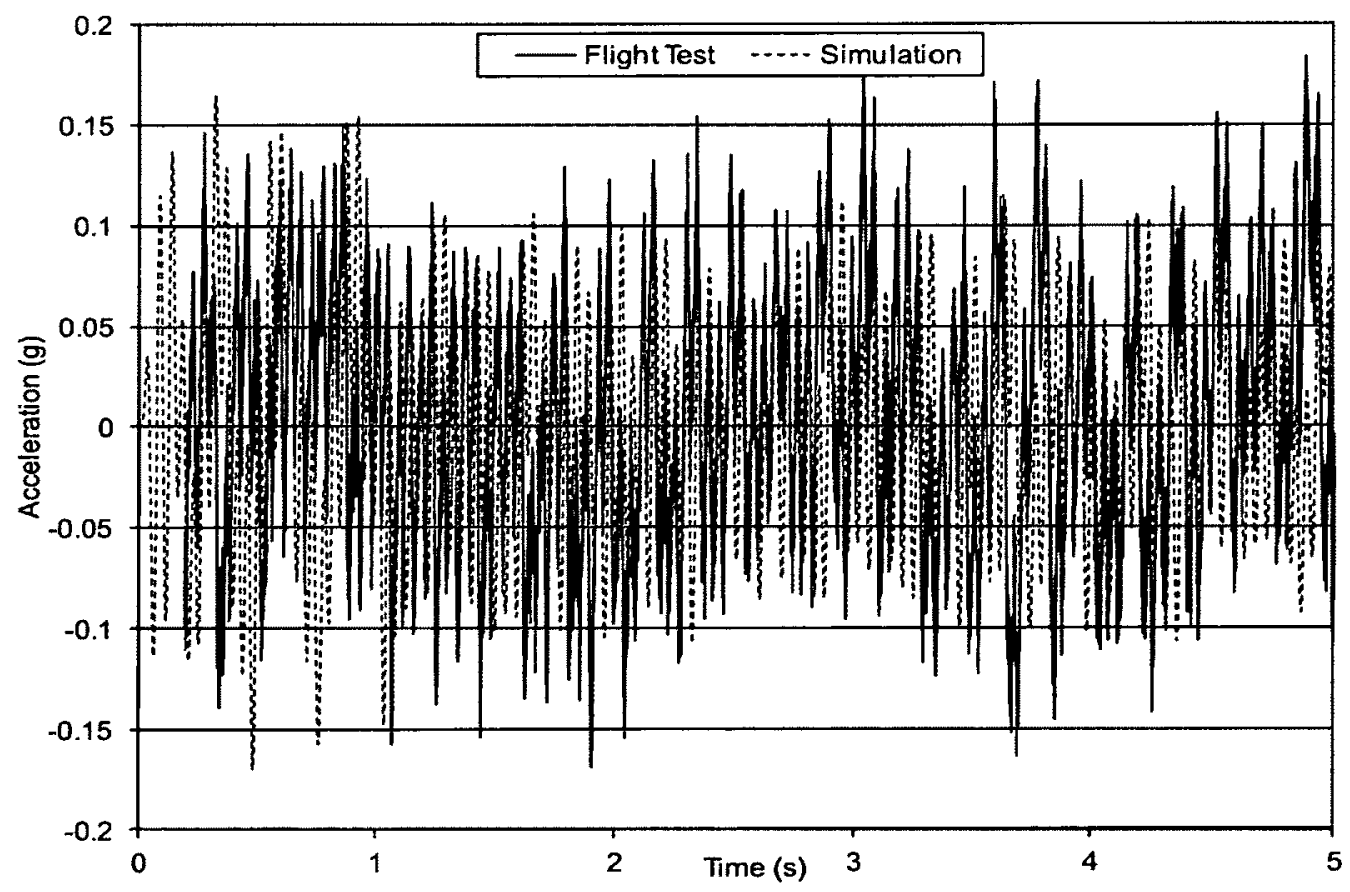

Figure 3.13: Time domain response comparison for simulation and flight test 
The vibration simulation using the biodynamic model was used to evaluate a variety of adaptive seat mount design options for the Bell-412 helicopter seat. The simulation results showed that in order to provide effective reduction in vibration in aircrew vibration levels, structural modification to the seat such as optimization of the cushion material impedance properties or modification to the seat frame design to avoid dynamic coupling should also be considered in addition to active approaches designed to counteract forced excitation due to rotor harmonics. These simulation results were taken into consideration when designing the retrofit adaptive mount system integrated with active control technology for the Bell-412 helicopter seat.

\subsection{Summary of the Modeling and Simulation}

A comprehensive experimental modal test was performed to extract the modes of the seat system to develop a simplified biodynamic model based on lumped parameter modeling technique for efficient vibration simulations. The experimental modal test performed using the base excited shaker tests was used to extract the dynamic characteristics of the helicopter seat with a strapped mannequin. The experimental modal analysis process identified several critical resonance modes of the seat structure that contributed to the aircrew vibration. The modal tests showed that the resonance modal frequencies varied with the aircrew weight as well as the input level. Such variation in modal parameters clearly justifies the need to develop an adaptive seat mount system using active control technology that could optimize vibration suppression under varying conditions.

An efficient lumped parameter model was developed to represent the dynamics of the seat structure as well as the aircrew for vibration simulations. The modal parameters extracted from the modal analysis were used to identify lumped parameters of the biodynamic model. The simulations performed using the model implemented on MATLAB based SIMULINK was used as an efficient means of evaluating various seat design options to mitigate helicopter aircrew exposure to harmful vibration. 


\section{Chapter 4}

\section{Adaptive Seat Mount Development and Verification}

This chapter provides details of the development process followed in the system design, development, fabrication and performance testing of the adaptive helicopter seat mount system to mitigate the vibration transmitted to the aircrew. The implemented adaptive seat mount design incorporated two stacked piezoelectric actuators to increase the bending stiffness to avoid the low frequency resonance while generating forces to counteract higher harmonic vibration peaks. The actuators were controlled using both a feed-forward control algorithm to counteract forced N/rev harmonics and a feedback control law to suppress the resonant vibration modes. The proof-of-concept adaptive helicopter seat mount was retrofitted on a full-scale Bell-412 aircrew seat and the vibration suppression performance was evaluated through extensive closed-loop tests on a mechanical shaker using representative helicopter floor vibration profiles.

\subsection{Adaptive Seat Mount Design}

The measurement of aircrew body vibration spectra from Bell-412 flight tests revealed high levels of vibration at rotor harmonics, particularly $4 / \mathrm{rev}$ and $2 / \mathrm{rev}$, while the vibration at $1 /$ rev measured on the aircrew was actually amplified. In addition, the extracted modal parameters from the experimental modal test of the Bell-412 seat with a strapped mannequin showed a structural bending mode of the system at $5.6 \mathrm{~Hz}$ which 
couples with the $1 / \mathrm{rev}$ rotor harmonic to generate vibration amplification in the low frequency regime. Therefore, the objective of the adaptive seat mount was not only to counteract forced vibration frequencies at rotor harmonics but also to increase the structural stiffness of the seat at critical locations in order to move the bending vibration mode away from the $1 / \mathrm{rev}$ rotor frequency. Based on these design criteria, struts were incorporated at the front of the seat, as shown in Figure 4.1, to support the seat pan on both sides in order to increase the bending stiffness of the seat. The mechanical design of the two struts connected the seat pan to the base of the L-shaped frame through interface attachments. This structural modification with additional struts was expected to increase the bending stiffness of the seat frame to move the bending mode at $5.6 \mathrm{~Hz}$ out of the range of major rotor harmonics. Shear pins could be integrated into the attachment mechanisms to allow for free seat pan movement downwards in case of a crash to meet the crashworthiness criteria of the helicopter seat design. In addition, these two struts were envisioned to incorporate actuators that could generate sufficient forces to counteract the transmission of vibration to aircrew. The mechanical design of the adaptive mount that retrofits the Bell-412 seat within the available footprint was expected to mitigate the vibration transmitted to the helicopter aircrew.

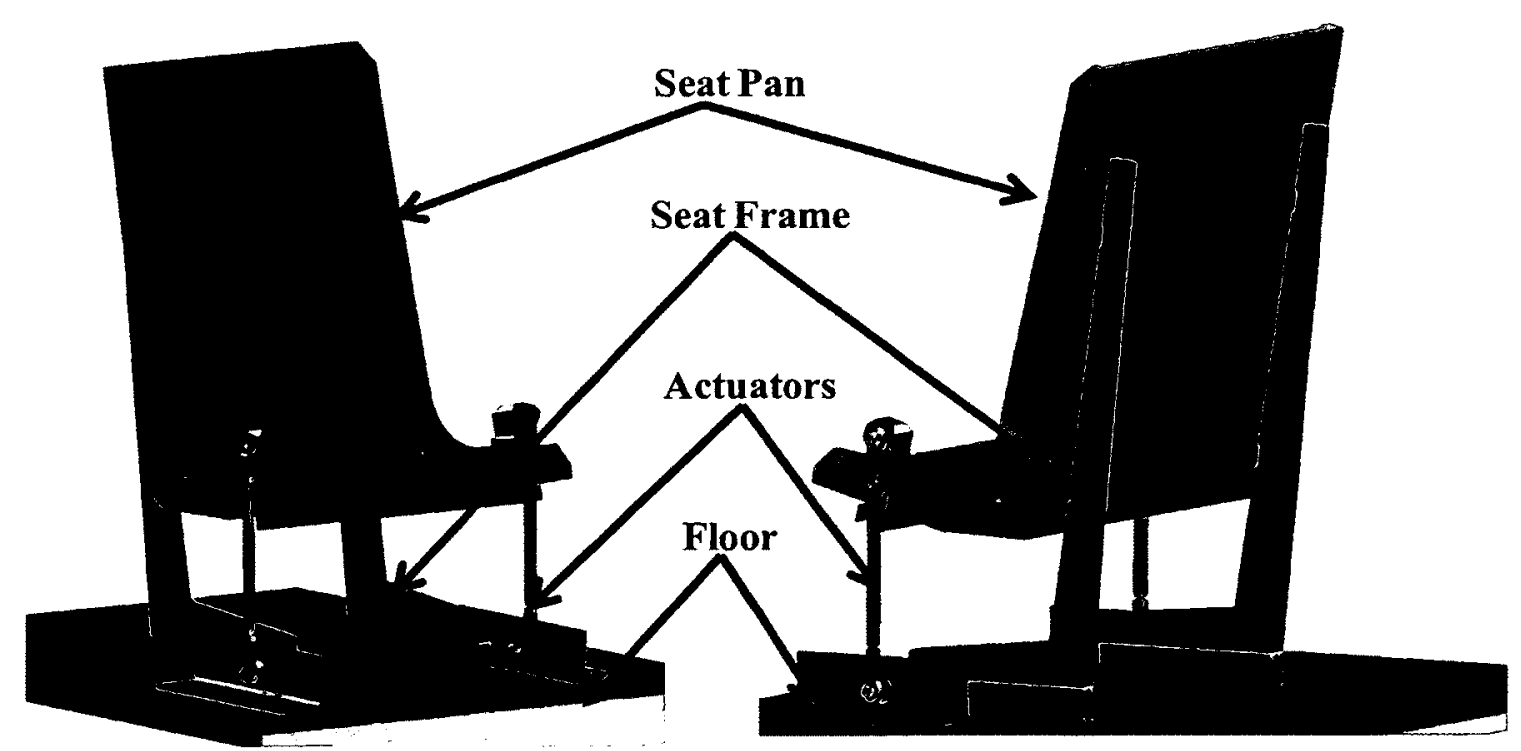

Figure 4.1: Mechanical design of the adaptive helicopter seat mount 


\subsection{Determination of Actuator Specifications}

The forced vibration input due to the $\mathrm{N} / \mathrm{rev}$ harmonic vibratory loads transmitted from the seat to the aircrew requires that the actuator used in the adaptive seat mount be capable of generating sufficient force levels to counteract the vibration. Compared to semi-active concepts which only vary the damping property of the seat mount, the active seat mount concept provides wider frequency band control capability and superior performance to suppress the forced vibration excitations. However, this approach requires the actuation system to provide large displacement and high force simultaneously. In addition, the mechanical characteristics of the actuators must be sufficient to increase the static bending stiffness of the seat frame in order to avoid the low frequency vibration amplification observed at $1 / \mathrm{rev}$ harmonic. Furthermore, the physical size of the actuator must be sufficiently small to fit within the small footprint around the seat installed in the helicopter cabin. Therefore, a compact actuation system was required for the integration of the adaptive seat mount system with the full-scale Bell-412 helicopter seat hardware.

\subsubsection{Estimation of Actuation Authority}

As part of the design process of the adaptive seat mount based on active control technology, an investigation was conducted through simulations to estimate the actuator authority requirement. The vibration simulation used the biodynamic model discussed in Chapter 2, implemented in the SIMULINK software platform, to estimate the required actuator authority. Helicopter seat vibration levels measured from flight tests, as discussed in Chapter 1, were used as the excitation input to the simulation model. The simulation was used to determine the actuator force and the displacement for several vibration conditions recorded during the flight tests, namely, ground, hover, translational lift and cruise flight at $120 \mathrm{kts}$ as well as $140 \mathrm{kts}$. The dynamic simulation was used to estimate the required control authority for two actuators arranged as active struts to reduce the aircrew helmet vibration by $50 \%$.

The simulation results revealed that the actuation authority required for the direct force control actuator varied with the operating conditions as listed in Table 4.1. As 
expected, the operating conditions that generate higher vibration levels demanded larger actuation authority to counteract the vibration. Analysis also showed that a high actuation stroke was also required in order to counteract the $1 / \mathrm{rev}$ harmonic vibration because it was located in the lower frequency range. Since the structural modification with additional struts was expected to mitigate the $1 /$ rev vibration amplification, the primary purpose of the actuators was to counteract the higher harmonic excitations.

Table 4.1: Actuation requirement based on flight conditions

\begin{tabular}{|c|c|c|}
\hline \multirow{2}{*}{ Flight Condition } & \multicolumn{2}{|c|}{ Estimated Actuator Authority } \\
\cline { 2 - 3 } & Force (N-rms) & Displacement (mm-rms) \\
\hline Grounding & 198.0 & 1.33 \\
\hline Hover & 160.2 & 1.06 \\
\hline Translational Lift & 214.7 & 0.46 \\
\hline 120 kts Cruise & 138.2 & 0.12 \\
\hline 140 kts Dive & 245.8 & 0.22 \\
\hline
\end{tabular}

\subsubsection{Selection of the Actuation Technology}

Several actuation technologies such as hydraulic, electrodynamic and linear motors were considered for the adaptive seat mount design. Based on the required actuation authority, structural stiffness and the size, stacked piezoelectric actuators were selected as the most suitable actuation technology for the adaptive seat mount application. The investigation showed that those stacked piezoelectric actuators are capable of providing the actuation stroke and force simultaneously at high frequency regimes in order to enable effective vibration suppression in helicopter seat application.

Piezoelectric material is one of the most widely used active materials with inherent electrical and mechanical coupling characteristics. Piezoelectric material develops electrical charge when strained and conversely develops strain when subjected to a voltage [117]. These piezoelectric materials are highly suitable for structural control application due to their high frequency response, linear performance at low electrical fields, high frequency operation and their ability to generate large actuation forces due to high stiffness. For these reasons, actuators based on piezoelectric materials are 
increasingly used in adaptive structures applications with active control technologies. The most common piezoelectric ceramic is lead zirconate titanate, $\mathrm{Pb}(\mathrm{Zr}, \mathrm{Ti}) \mathrm{O}_{3}$, commonly known as PZT [118]. The induced strain coefficient $d$ is the most relevant electromechanical coupling property of PZT for actuation and sensing applications [119], and is given by the expression:

$$
d=\left(\frac{S}{E}\right)_{T}=\left(\frac{D}{T}\right)_{E}
$$

In actuator mode, $d(\mathrm{~m} / \mathrm{V})$ is the strain $S$ developed per unit applied electric field $E$, and in sensor mode, $d(\mathrm{C} / \mathrm{N})$ is the electrical charge $D$ generated per unit applied mechanical stress $T$, subscripts denote the parameter held constant.

Table 4.2: Bulk piezoelectric properties [120]

\begin{tabular}{|c|c|c|c|c|}
\hline Property Name & PZT-4 & PZT-5A & PZT-5H & PZT-8 \\
\hline Strain coefficient $-\mathrm{d}_{31}$ & $-122 \times 10^{-12}$ & $-171 \times 10^{-12}$ & $-274 \times 10^{-12}$ & $-97 \times 10^{-12}$ \\
\hline Strain coefficient $-\mathrm{d}_{33}$ & $285 \times 10^{-12}$ & $374 \times 10^{-12}$ & $593 \times 10^{-12}$ & $250 \times 10^{-12}$ \\
\hline Closed circuit stiffness $-Y_{11}$ & $82 \mathrm{GPa}$ & $61 \mathrm{GPa}$ & $62 \mathrm{GPa}$ & $87 \mathrm{GPa}$ \\
\hline Closed circuit stiffness $-Y_{33}$ & $61 \mathrm{GPa}$ & $53 \mathrm{GPa}$ & $48 \mathrm{GPa}$ & $74 \mathrm{GPa}$ \\
\hline Density & $7600 \mathrm{~kg} / \mathrm{m}$ & $7700 \mathrm{~kg} / \mathrm{m}$ & $7450 \mathrm{~kg} / \mathrm{m}$ & $7500 \mathrm{~kg} / \mathrm{m}$ \\
\hline
\end{tabular}

PZT material exhibits piezoelectric properties when randomly oriented electric dipoles are aligned in a common direction in a process called poling. An electrical field is applied during the poling process to orient the dipoles in the direction of the electrical field. The magnitude of the coupling coefficient may vary with the direction of electrical and mechanical application. In general, the ' 3 ' direction is defined as the direction of poling while ' 1 ' and ' 2 ' are the perpendicular directions. Thus the $d_{31}$ coefficient relates strain developed in the ' 1 ' direction due to the electric field applied along the direction of poling. This $d_{31}$ coefficient is also known as the secondary or transverse piezoelectric effect. In contrast, the $d_{33}$. coefficient relates to the strain developed in the direction of poling to the electric field applied in the same direction. This $d_{33}$. coefficient is known as the primary or longitudinal piezoelectric effect. Typically the transverse $\mathrm{d}_{31}$. piezoelectric effect is approximately half of the primary $\mathrm{d}_{33}$. effect in magnitude. The material 
properties of commercially available bulk piezoelectric material relevant to structural control applications are summarized in Table 4.2.

Although the strain capability of bulk piezoelectric material is generally less than $0.1 \%, \mathrm{PZT}$ is increasingly used as effective structural actuators because of its ability to generate significantly high mechanical force due to the high nominal stiffness. The advantages of piezoelectric materials include high bandwidth operation, compatibility with simple power amplifiers and compact physical size compared to hydraulic systems. The interior of the piezoelectric actuator, shown in Figure 4.2(a), is a multilayered stack of thin PZT wafers electrically connected in parallel. The thinner the wafer, the lower the voltage required for maximum expansion of the actuator. Each thin wafer is actuated using the primary induced strain $d_{33}$ coefficient and several wafers are mechanically arranged in series to increase the actuator stroke. The multilayer piezoelectric stack actuators which produce high displacements are longer in length but generate large discrete actuation forces for a variety of structural control applications [121]. For example, stack actuators have been integrated into truss structures for control vibration $[122,123]$, used to counteract helicopter rotor vibration by powering a discrete trailing edge flap $[124,125,126]$, and applied to mitigate the transmission of helicopter gearbox vibration through the mounting structure $[127,128]$.

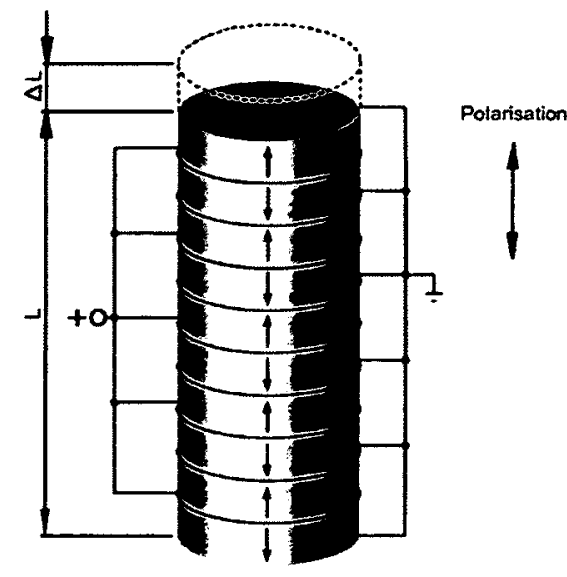

(a)

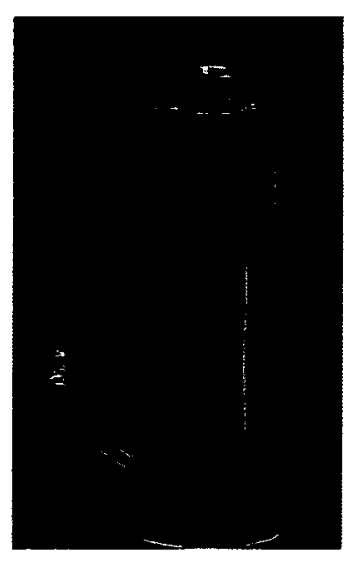

(b)

Figure 4.2: (a) Interior multilayered stack (b) Selected piezoelectric actuators 
In the proof-of-concept adaptive seat mount hardware design, the Pst 150/14/200 VS20 stacked piezoelectric actuators manufactured by PIEZOMECHANIK GmbH, as shown in Figure 4.2(b), were selected for the two active struts. The multilayered piezoelectric stack is preloaded in a ruggedized stainless steel casing for protection. The length of the actuator is $200 \mathrm{~mm}$ and the diameter of the actuator is $20 \mathrm{~mm}$. The free stroke of $0.2 \mathrm{~mm}$ was generated at the moving head as the operating voltage varied from $0 \mathrm{~V}$ to $150 \mathrm{~V}$. The actuator was powered by a LE $150 / 200-3$ high voltage amplifier manufactured by PIEZOMECHANIK GmbH capable of generating $\pm 150 \mathrm{~V}$ with a peak current of 2 A. Although the actuator is rated to generate a maximum force of $7000 \mathrm{~N}$, the pre-stress of $1000 \mathrm{~N}$ limits the maximum tensile force that can be safely applied to the moving head. Based on simulation results shown in Table 4.2, the force and stroke capabilities of the selected actuator was sufficient to reduce vibration by $50 \%$ at the cruise speed of $120 \mathrm{kts}$, the longest operating condition in many helicopter missions. This stacked piezoelectric actuator pair provided the required actuation authority and sufficient structural stiffness, due to the casing, within a small physical size, and therefore is suitable as the actuation mechanism of the adaptive helicopter seat mount design.

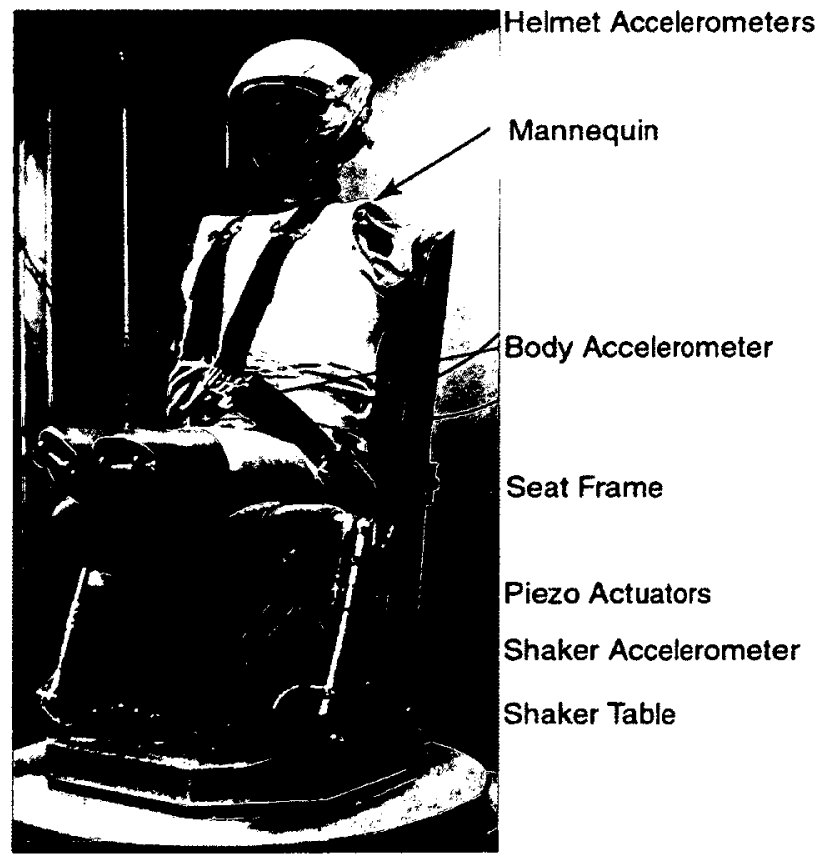

Figure 4.3: Adaptive seat mount integrated on full-scale Bell-412 seat 


\subsection{System Identification of Integrated Seat Hardware}

The proof-of-concept adaptive seat mount, which included two stacked piezoelectric actuators integrated as active struts, retrofitted on a full-scale Bell-412 helicopter seat is shown in Figure 4.3. The two struts were connected to the sides of the seat pan and attached to the L-shaped frame through precisely designed interface attachment mechanisms to properly control the tilt angle. The tilt angle of the piezoelectric actuators were set carefully to allow the adaptive mount to control the seat vibrations in both the vertical and the fore-aft directions simultaneously. The integrated seat hardware was placed on the mechanical shaker table with the $95^{\text {th }}$ percentile mannequin strapped to the seat to perform experimental system identification tests.

In order to evaluate the effectiveness of the two additional struts to modify the dynamic properties of the Bell-412 seat, an experimental modal test was conducted on the retrofitted hardware with the seated mannequin as previously discussed in Section 3.1. It is important to note that the actuators were not active during the test but they behaved as additional mechanical struts incorporated on the structure to increase the seat bending stiffness. The major vibration modes of the modified seat extracted from experimental modal analysis are shown in Figure 4.4.

The extracted modal parameters showed that first seat bending mode at $5.6 \mathrm{~Hz}$ was shifted to beyond $50 \mathrm{~Hz}$ due to the addition of the two struts. Such increase in the seat bending stiffness avoids the low frequency vibration amplification that was induced by the dynamic coupling of the bending mode with the $1 /$ rev rotor harmonic. The second seat bending mode originally located at $43.2 \mathrm{~Hz}$ was also increased beyond $50 \mathrm{~Hz}$. Therefore, it was expected that the vibration due to these resonances be reduced after the modifications of the seat with these additional struts to mitigate aircrew vibration levels. However, the vibration modes at $9.7,10.8,16.6$ and $19.7 \mathrm{~Hz}$ were virtually unchanged due to this structural modification to the seat.

The actuation performance of the adaptive seat mount consist of the stacked piezoelectric actuators integrated into the Bell-412 were also evaluated using the standard system identification process. Actuating the piezoelectric actuators using sine tone excitations at rotor harmonic frequencies showed that the adaptive mount system was 
able to generate sufficient vibration on the mannequin helmet to counteract the vibration transmitted through the floor.
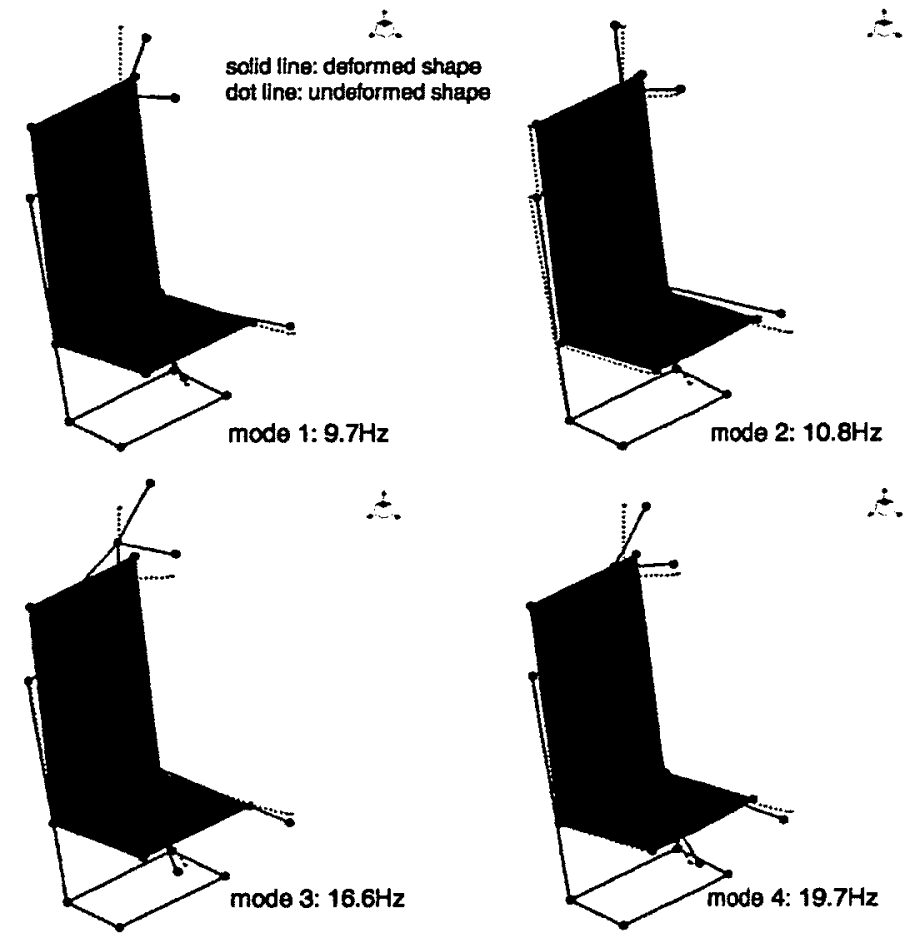

Figure 4.4: Modes of the Bell-412 seat integrated with active struts

In addition, system identification was performed by experimentally measuring the transfer functions to the mannequin helmet location in the fore-aft direction from the mechanical shaker input and the actuation of piezoelectric struts, commonly known as the control path $H(z)$. This was achieved by applying a linearly sweeping sinusoidal signal from 1 to $50 \mathrm{~Hz}$ at a rate of $1 \mathrm{~Hz} / \mathrm{sec}$ to the piezoelectric actuators and the mechanical shaker while the accelerometer response at the helmet location was synchronously recorded in with the input signal. Using the experimentally measured transfer functions, the System Identification Toolbox provided with the MATLAB software was used to generate a linear parametric model in the State-Space form. The Bode diagram obtained from the identified State Space model is shown in Figure 4.5. It demonstrated that the vertical vibration input from the mechanical shaker transmitted through the Bell-412 seat 
primarily excited the localized mannequin helmet mode at $9.7 \mathrm{~Hz}$ and the vertical mannequin body mode at $16.6 \mathrm{~Hz}$. It is important to note that the two stacked piezoelectric struts were configured in parallel as one control input channel in the present investigation. This parallel configuration provided effective control authority over the vibration modes at $9.7 \mathrm{~Hz}$ and $19.7 \mathrm{~Hz}$. As discussed in detail in Chapter 3, the modal parameters vary depending on the mannequin mass, input level, posture, et cetera. Therefore, adaptive control laws are required to perform on-line identification of the transfer functions for effective vibration suppression of the aircrew body vibrations when implemented in the operational situation.

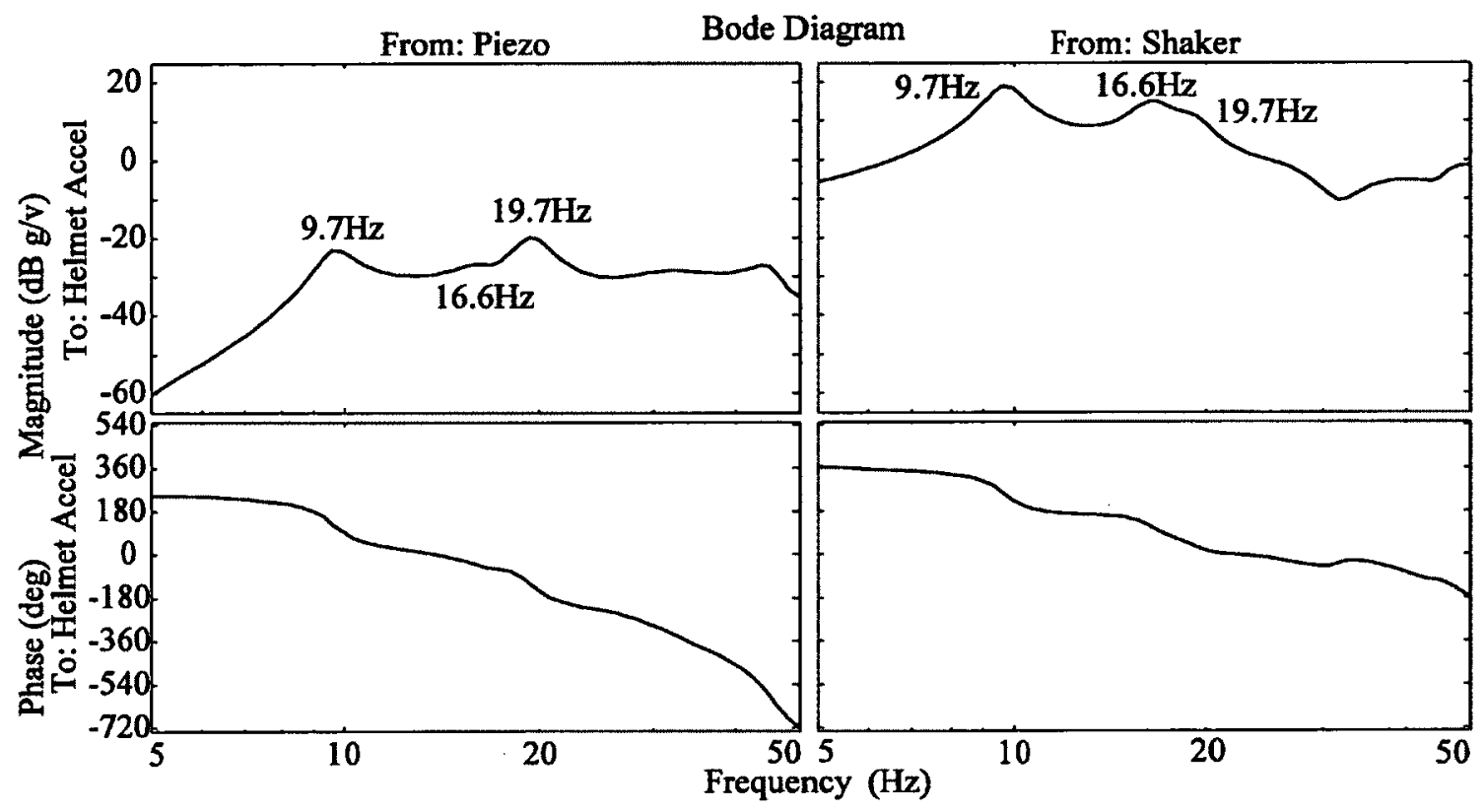

Figure 4.5: Transfer functions from piezoelectric actuator and mechanical shaker

\subsection{Adaptive Control Law Development}

Flight tests, discussed in Chapter 2, revealed that the aircrew vibration spectra are dominated by $\mathrm{N} / \mathrm{rev}$ harmonics of rotor dynamic load. In particular, the measured aircrew body vibration was dominated by the $1 / \mathrm{rev}$ and $4 / \mathrm{rev}$ harmonic peaks while the contribution from $2 / \mathrm{rev}$ and $6 / \mathrm{rev}$ harmonics was minor. Therefore, the control force 
generated by the stacked piezoelectric actuators of the adaptive mount was required to primarily counteract the forced vibration loads at $\mathrm{N} / \mathrm{rev}$ harmonics in order to provide effective vibration suppression for the aircrew body. Secondarily, critical resonant modes of the seated aircrew that are being excited due to random vibration were required to be suppressed by the actuator forces. With an effectively designed adaptive control algorithm, the adaptive seat mount system was able to provide vibration suppression of the $\mathrm{N} / \mathrm{rev}$ harmonics as well as control of the resonant structural modes to significantly reduce the vibration transmitted to helicopter aircrew.

The performance objective of the control algorithm developed for this investigation was to reduce the aircrew head vibration. In particular, the accelerometer that measured the helmet vibration in the fore-aft direction was selected as the error sensor, $\mathrm{e}(\mathrm{k})$, while accelerometers at the helmet in the vertical direction and the aircrew abdomen were monitored for overall system performance evaluation purposes. Both piezoelectric actuators on either side of the seat were electrically configured in parallel to operate with a single control input channel. This configuration enabled the implementation of a Single-Input-Single-Output (SISO) control system to control the adaptive helicopter seat mount system.

\subsubsection{Adaptive Notch Concept to Control Harmonics}

There are several control approaches that have been used in the past to suppress harmonic disturbances with varying degrees of success. Among the control strategies to eliminate harmonic disturbances, Morgan and Stanford developed a promising controller design approach that introduced "notches" at synchronous frequencies [129] to control harmonics. For narrow-band applications, a reliable sinusoidal reference signal is used to configure the Least Mean Square (LMS) as an adaptive notch filter by removing the primary spectral components within a narrow band centered about the reference frequency. Chen et al have shown previously that an adaptive notch controller implemented with a Filtered-x Least Mean Square (FLMS) algorithm with a suitable reference signal was able to cancel the helicopter rotor vibration [130]. This previously published principle of a FLMS algorithm formulation shown in Figure 4.6 was used for 
the current investigation [131]. It is important to note that discrete time implementation of the controller and plant model was used for the controller development.

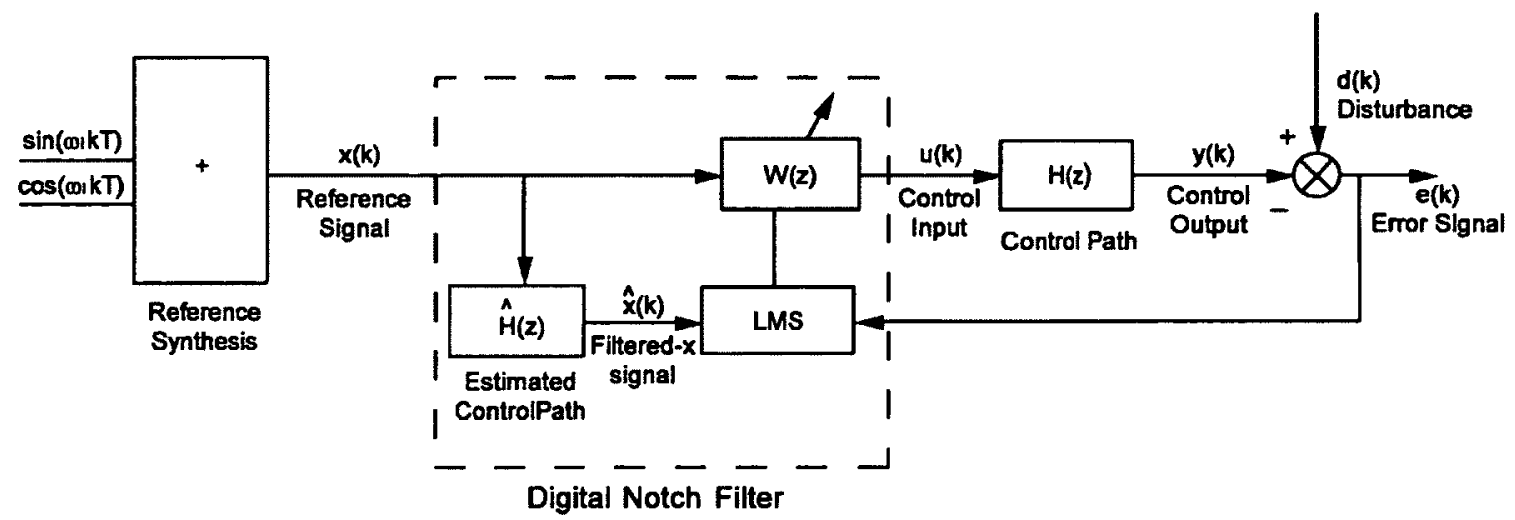

Figure 4.6: Adaptive notch controller diagram

Here, the disturbance $d(k)$ represents the external excitation forces such as the $\mathrm{N} / \mathrm{rev}$ harmonics and random excitation of the aircrew. The error signal $e(k)$ is the acceleration at the aircrew helmet location used to satisfy the objective of the controller. The reference signal, control input and control output are denoted by $x(k), u(k)$ and $y(k)$, respectively. Since the disturbance frequency $\omega_{i}$ is usually unknown to designers, an estimate of the frequency, $\hat{\omega}_{i}$, is necessary. Thereafter, the reference signal is synthesized as a combination of sinusoids at the estimated frequencies as following:

$$
x(k)=\sum_{i=1}^{0} \cos \hat{\omega}_{i} k T=\sum_{i=1}^{o} \frac{1}{2}\left[e^{j \tilde{\omega}_{i} k T}+e^{-j \hat{\omega}_{i} k T}\right]
$$

where $i=1 \ldots o$ while the $o$ is the number of frequencies to be included in the reference signal. The reference signal is filtered with magnitude and delay through the control path. Assuming the transfer function of the control path, estimated as $\hat{\mathrm{H}}(\mathrm{z})$, generates the weights of the FIR filter for the control path transfer function $h(k)$, the filtered signal can be expressed as: 


$$
\hat{\mathrm{x}}(\mathrm{k})=\mathrm{h}(\mathrm{k})^{*} \mathrm{x}(\mathrm{k})
$$

The filtered signal $\hat{x}(k)$ is then adapted by the LMS algorithm as discussed previously in the published references [132]. The $\mathrm{W}(\mathrm{z})$ provides the controller coefficients of the iterative weights $w(k)$ in order to generate the adapted control input $u(k)$ using the reference signal $\mathrm{x}(\mathrm{k})$ as shown below;

$$
\mathrm{u}(\mathrm{k})=\mathrm{w}(\mathrm{k}) * \mathrm{x}(\mathrm{k})
$$

The control output signal $y(k)$ denoted in the discrete domain as $Y(z)$ is generated by the FLMS algorithm and expressed as follows:

$$
\begin{aligned}
& Y(z)=\frac{n \mu}{4} E(z) \sum_{i=1}^{m}|\hat{H}(z)|^{2}\left[\frac{1}{z e^{-j \hat{\omega}_{i} T}-1}+\frac{1}{z e^{j \hat{\omega}_{i} T}-1}\right] \\
& +\left.\sum_{i=1}^{m} \sum_{k=1}^{m} \frac{\mu|\hat{H}(z) \| \hat{H}(z)|}{4} \frac{\sin \left(\frac{\hat{\omega}_{i}-\hat{\omega}_{k}}{2} n T\right)}{\sin \left(\frac{\hat{\omega}_{i}-\hat{\omega}_{k}}{2} T\right)}\right|_{i \neq k} \\
& +\sum_{i=1}^{m} \sum_{k=1}^{m} \frac{\mu|\hat{H}(z) \| \hat{H}(z)|}{4} \frac{\sin \left(\frac{\hat{\omega}_{i}+\hat{\omega}_{k}}{2} n T\right)}{\sin \left(\frac{\hat{\omega}_{i}+\hat{\omega}_{k}}{2} T\right)}
\end{aligned}
$$

where, $E(z)$ is the error signal in discrete domain. Assuming that no error in the frequency estimate is present and the control path is Linear Time Invariant (LTI), the first term in the control signal is time-invariant, which produces notches at the estimated frequencies, while terms 2 and 3 are time-varying. However, if the length of the FIR filter, $\mathrm{n}$, is comparatively long and the convergence coefficient, $\mu$, is relatively small, the time-varying components will be small and can be neglected. As a result, an equivalent filter with a notch at frequency $\hat{\omega}_{\mathrm{i}}$ will be obtained. The equivalent closed-loop transfer function $\mathrm{C}(\mathrm{z})$ between the harmonic reference signal and the error signal on the form of a classical bandpass filter, with notches at the reference frequencies, can be obtained as: 


$$
C(z)=\frac{1}{1+\frac{n \mu}{4} \sum_{i=1}^{m}|\hat{H}(z)|^{2}\left[\frac{1}{e^{-j \hat{\omega}_{i} \mathrm{~T}}-1}+\frac{1}{e^{j \hat{\omega}_{i} \mathrm{~T}}-1}\right]}
$$

If the disturbance frequency varies slowly with time, it is necessary to identify the variation in frequency in real time to update the reference signal for the FLMS controller, such that a tuneable notch filter can be formed adaptively.

However, due to the nature of identification, a frequency estimate error between $\hat{\omega}_{\mathrm{i}}$ and $\omega_{\mathrm{i}}$ is inevitable. As a result, the modulation between the error signal $\mathrm{e}(\mathrm{k})$ and the filtered reference signal $\hat{x}(k)$ will lead to an oscillation in the weights of the FLMS algorithm. If the estimate error is small so that the $\omega_{i}-\hat{\omega}_{i}$ component in the controller weight is stronger than $\omega_{i}+\hat{\omega}_{i}$, the notch filter effect would also be present. Therefore, an efficient and accurate frequency estimation method is essential for the algorithm to track the shift in the disturbance frequencies.

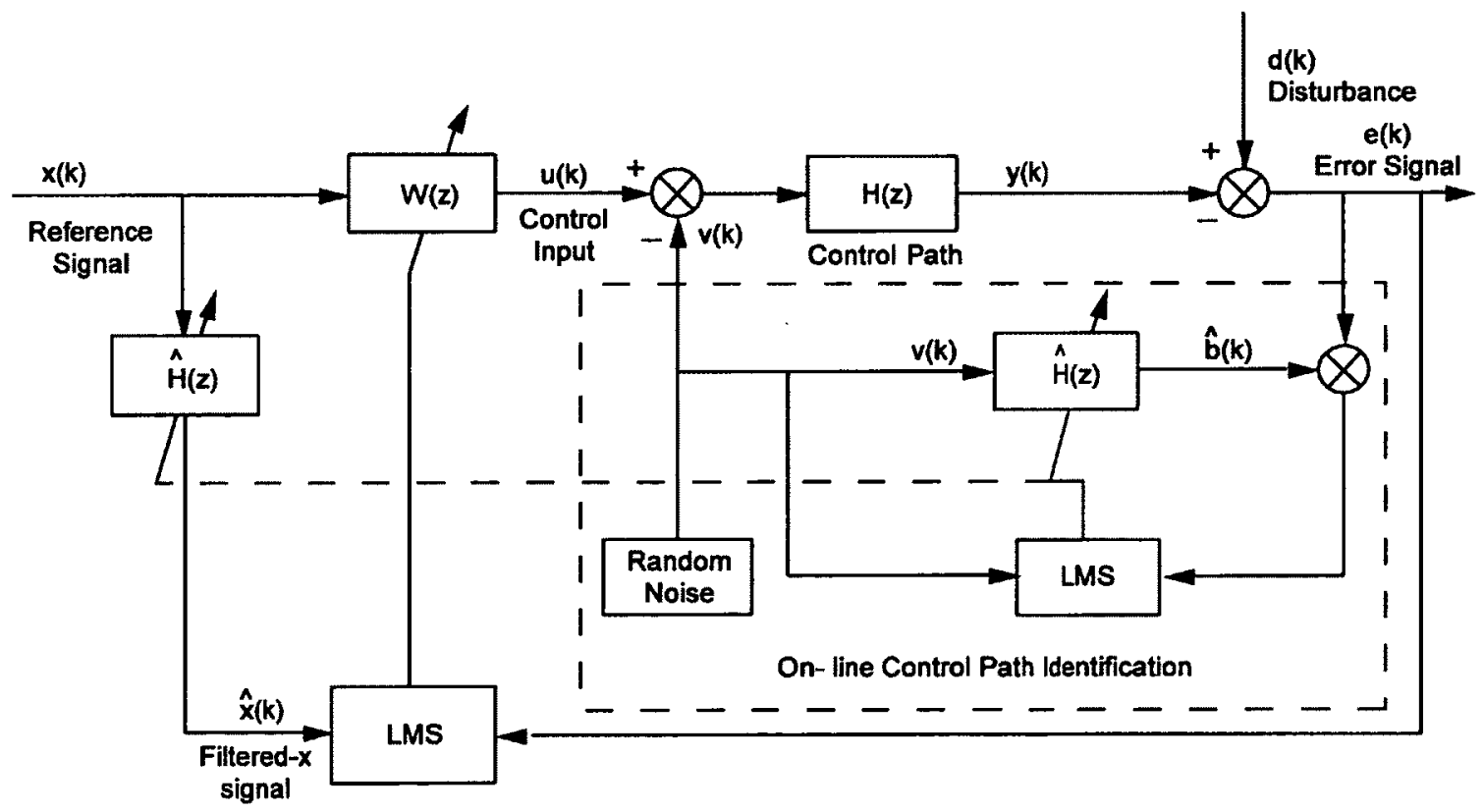

Figure 4.7: On-line identification of control path 


\subsubsection{Control Path Identification Method}

As mentioned previously, the aircrew vibration frequency varies with the flight conditions, aircrew weight, posture and many other factors. Therefore, the transfer function of the control path, $\mathrm{H}(\mathrm{z})$, is time-varying and therefore needs to be estimated online. The block diagram of a SISO adaptive feed-forward control law in Figure 4.7 shows the identification process for the control path, represented by the transfer function between the stacked piezoelectric actuators and the accelerometer at the helmet location. By introducing white noise, $v(k)$, into the control input as the identification excitation, the contribution of this excitation $b(k)$ is measured by the error sensor such that

$$
b(k)=h(k) * v(k)
$$

If the white noise and control signal are not correlated, an estimate of the control path $\hat{\mathrm{H}}(\mathrm{z})$ can be obtained using the LMS algorithm. Assuming $\hat{\mathrm{H}}(\mathrm{z})$ is represented by a FIR filter, the weights $w_{h}(k)$ can be obtained through iteration as follows:

$$
W_{h}(k+1)=W_{h}(k)+\mu_{h} v(k)[(b(k)+d(k)-h(k) * u(k)-\hat{b}(k)]
$$

By defining

$$
\begin{gathered}
\hat{b}(k)=\hat{h}(k) * v(k) \\
o(k)=d(k)-h(k) * u(k)=d(k)-h(k) * w(k) * x(k)
\end{gathered}
$$

the adaptive law governing the weights of the control path is expressed as follows:

$$
W_{h}(k+1)=W_{h}(k)+\mu_{h} v(k)[(b(k)+o(k)-\hat{b}(k)]
$$

Compared to the off-line system identification method, it can be shown that $o(k)$ represents a signal from the feed-forward controller. This parameter interferes with the 
control path identification process, which may slow down the convergence speed and even lead to a divergence of the process [133].

A compensation applied to $o(k)$ as shown in Figure 4.8 is able to improve the efficiency in the control path identification process. Because $o(k)$ is a narrow band signal that consists of multiple harmonic frequencies, it can be cancelled by introducing another adaptive filter, $G(z)$, which has multiple notches at the corresponding frequencies in order to eliminate the interference in control path identification.

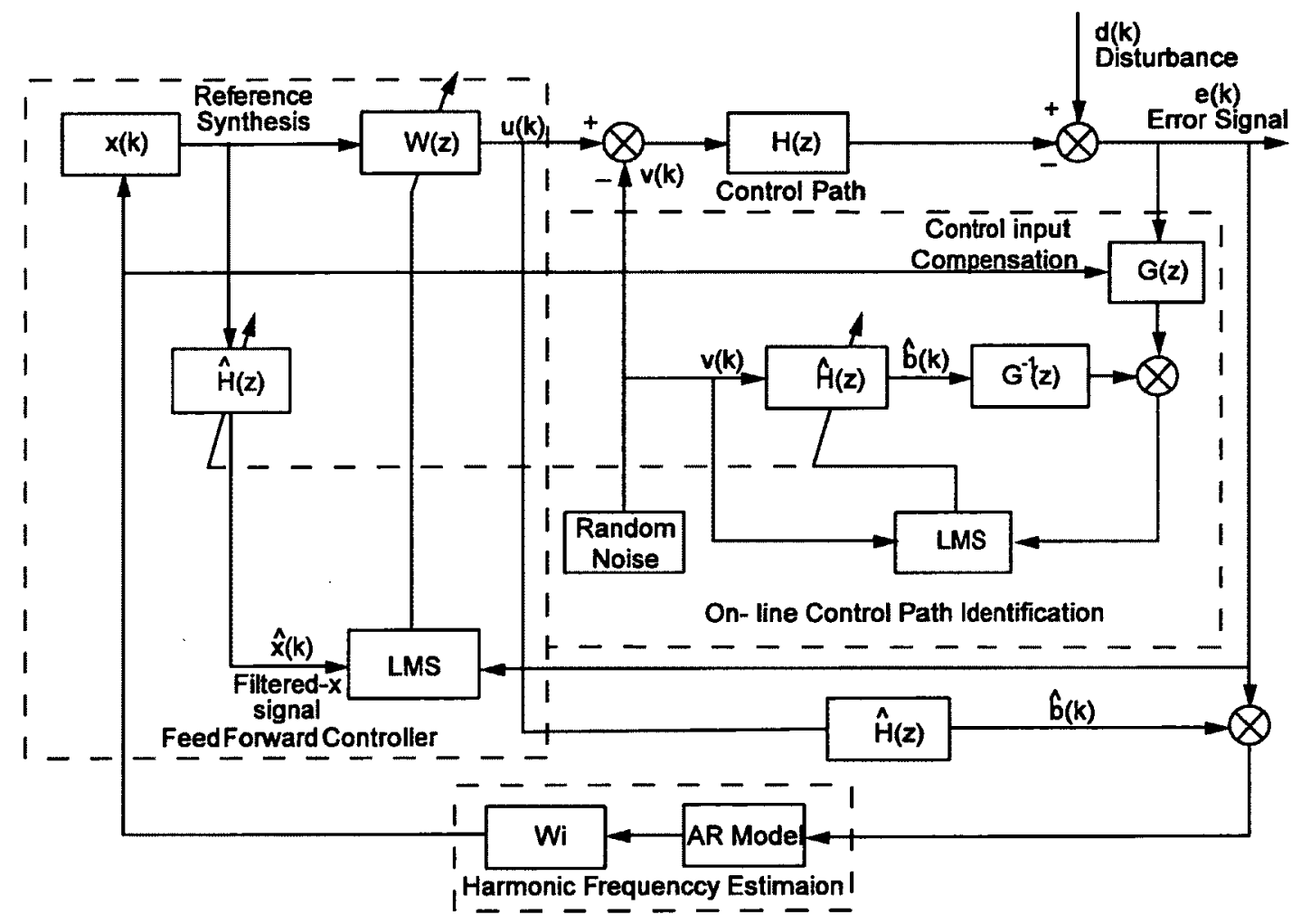

Figure 4.8: Compensator for control path identification

The filter $G(z)$ can be designed for frequency $\hat{\omega}_{i}$ as seen from Figure 4.9. Only two weights are needed to cancel one frequency, which can be expressed as

$$
\begin{aligned}
& \mathrm{w}_{\mathrm{G}, 1}(\mathrm{k}+1)=\mathrm{w}_{\mathrm{G}}(\mathrm{k})+\mathrm{c} \mu_{1} \mathrm{ee}(\mathrm{k}) \cos \left(\mathrm{k} \hat{\omega}_{\mathrm{i}} \mathrm{T}+\Phi_{\mathrm{i}}\right) \\
& \mathrm{w}_{\mathrm{G}, 2}(\mathrm{k}+1)=\mathrm{w}_{\mathrm{G}, 2}(\mathrm{k})-\mathrm{c} \mu_{1} \operatorname{ee}(\mathrm{k}) \sin \left(\mathrm{k} \hat{\omega}_{\mathrm{i}} \mathrm{T}+\Phi_{\mathrm{i}}\right)
\end{aligned}
$$


where ee(k) is error signal of the adaptive notch filter, $\mu_{1}$ is the convergence coefficient of the compensator and $c$ is the amplitude of the synthesized reference signal. The equivalent transfer function of the compensator filter is as follows:

$$
G(z)=\frac{z^{2}-2 z \cos \omega_{0} T+1}{z^{2}-2\left(1-\mu_{1} c^{2}\right) z \cos \omega_{0} T+1-2 \mu_{1} c^{2}}
$$

As long as a reliable reference signal that includes controllable sinusoidal interference is available, the adaptive notch filter automatically creates a notch over each sinusoid and tracks its frequency drift. Therefore, this adaptive notch filter provides a simple and effective alternative to other methods of tracking and eliminating $\mathrm{N} / \mathrm{rev}$ harmonics in the helicopter seat vibration control application.

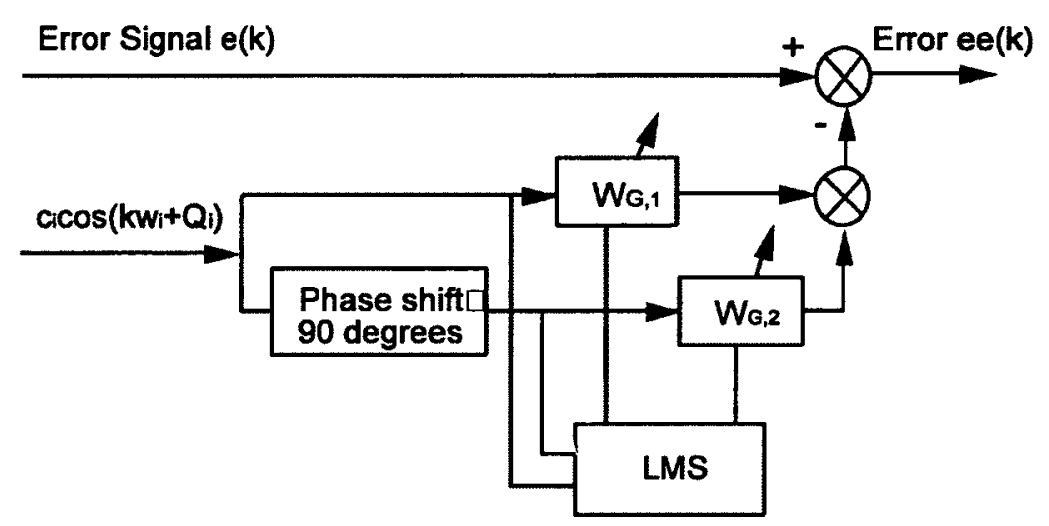

Figure 4.9: Adaptive notch filter

\subsubsection{Reference Signal Synthesis Method}

A reliable reference signal is very important for the successful application of the FLMS algorithm for vibration control because it guarantees that the controller is stable in the closed-loop system. Among a number of possible approaches, the signal synthesis method based on digital signal processing techniques was selected because it avoided introduction of feedback loops in the control path. This reference signal synthesis approach guarantees that the controller is purely feed-forward and stable, and also 
assumes that the variation of disturbance frequency is slow, which is appropriate for helicopter vibration control applications.

An accurate and efficient estimate of the disturbance frequency is necessary to achieve reliable cancellation of harmonic vibrations transmitted to the aircrew through the seat. There are two approaches, namely, non-parametric and parametric, that were used to estimate the frequency content of a signal based on a finite set of data observations. The non-parametric methods are based on the Fast Fourier Transform (FFT) technique. Since the accuracy of this method depends on the length of the data series, comparatively long data series are required to obtain an accurate estimate. Therefore, this type of FFT based frequency estimation method is not suitable for realtime applications. The high computational burden of FFT analysis is another challenge for real-time controller implementation. An alternative approach is to use a parametric method that is based on digital signal processing techniques. Although it is relatively fast, this approach provides high resolution frequency estimates despite short data series, making it an attractive approach for the present adaptive control algorithm. The parametric method uses a predetermined model to estimate the frequency components of the signal. A covariance autoregressive (AR) method is adopted in the present investigation. The AR model can be expressed as:

$$
\mathrm{P}\left(\mathrm{z}^{-1}\right)=\frac{\mathrm{K}}{\mathrm{A}\left(\mathrm{z}^{-1}\right)}=\frac{\mathrm{K}}{1+\mathrm{a}_{2} \mathrm{z}^{-2}+\mathrm{a}_{3} \mathrm{z}^{-3}+\cdots+\mathrm{a}_{\mathrm{p}+1} \mathrm{z}^{-\mathrm{q}+1}}
$$

where, $q, K$ and $a_{i}$ are model order, scalar gain and coefficients, respectively. Due to the fact that the poles of the AR model correspond to the estimated frequencies, the frequencies can be calculated from the AR polynomial once an estimate of the AR model coefficients is obtained. Therefore, a reference signal for the adaptive algorithm could be synthesized as a linear combination of multiple sinusoids as shown in Eq. 4.2.

An effective adaptive controller to counteract helicopter rotor harmonics was successfully developed with such a synthesized reference signal. Since the harmonic frequencies in aircrew excitation are estimated on-line and selectively included in the 
reference signal, the algorithm does not only suppress vibration modes selectively, but also tracks the change in the vibration spectra. In this investigation, the floor vibration that excites the seat frame was measured directly by an accelerometer and used as the reference signal in the adaptive feed-forward control law. This approach guaranteed that the FLMS control system was stable in the closed-loop system.

\subsubsection{Stability of the FLMS Control Algorithm}

The stability of the FLMS control algorithm has been demonstrated in a variety of situation even when the coefficients change within the time scale associated with the dynamic response of the forward path [134]. The statistical expectation operator is used in order to investigate the stability of the FLMS algorithm, making the problem deterministic similar to the LMS formulation. This was performed by diagonalizing the algorithm in Eq. 4.9 using the orthonormal transformation to express it as a set of independent scalar equations [135]. In order to ensure that the action of the LMS algorithm is stable, the maximum value for the convergence coefficient, $\mu$, step length should be given approximately by:

$$
\mu<\frac{2}{\mathrm{E}\left[\mathrm{x}^{2}(\mathrm{z})\right]}
$$

where, $\mathrm{E}$ is the expected value operator.

However, in the case of the FLMS algorithm, Elliot et al. [136] have found that the maximum step length $\mu$ not only depends on the length of the adaptive filter and filtered reference signal but also on the delays in the forward path $C$. If the filtered reference signal $\hat{x}(z)$ is a white noise process it has thus been found that an upper limit for the step length $\mu$ is given by:

$$
\mu_{\max } \approx \frac{2}{\mathrm{E}\left[\hat{\mathrm{x}}^{2}(\mathrm{z})\right](\mathrm{M}+\delta)}
$$


where, $M$ is the number of filter coefficients and $\delta$ is the overall delay in the forward path in samples. In practice, the FLMS algorithm exhibits stable behaviour even when the coefficients change within the time scale associated with the dynamic response of the forward path $[134,135]$.

\subsubsection{Simulation of the Adaptive Notch Control Algorithm}

A numerical simulation was conducted using the SIMULINK tool provided as an extension in the MATLAB software to verify the performance of the adaptive notch control algorithm for the helicopter seat mount application. The control objective was to mitigate the vibration level at the pilot helmet because the associated dynamic load was assumed as the primary source that contributed to the severity of the aircrew neck and back injuries. Therefore, the accelerometer that measured the helmet vibration in the fore-aft direction was used as the error signal for minimization.

Simulation of the vibration control using the aforementioned adaptive feedforward control law required knowledge of both the dynamics of the secondary control path and the vibration levels as measured by the error sensor. The primary control path represented the vibration disturbance input to the aircrew, which is generally complex in nature. In this simulation, the vibration levels measured at the pilot helmet location during flight test was directly used as the disturbance signal. As discussed previously, the secondary control path was experimentally identified as shown in Figure 4.5. The helicopter floor and aircrew helmet vibration spectra recorded from the flight test was used as the reference data for this simulation to evaluate controller performance. Studied flight conditions included ground, hover, and forward cruise conditions.

It is also important to note that the numerical simulation was conducted primarily to provide a qualitative feasibility evaluation of the adaptive control approach to mitigate the aircrew vibration levels. Vibration simulation results predicted for the fore-aft direction of the aircrew helmet for the translational flight condition are shown in Figure 4.10. The impact of the proposed mechanical struts on the structural dynamics of the seat structure was not considered in this simulation. Therefore, the "no control" curve represented the aircrew helmet vibration level without additional stiffness due to 
mechanical struts, while the "control" curve represented the predicted helmet vibration level with the actuation forces generated by the selected piezoelectric actuators to counteract the input vibration.

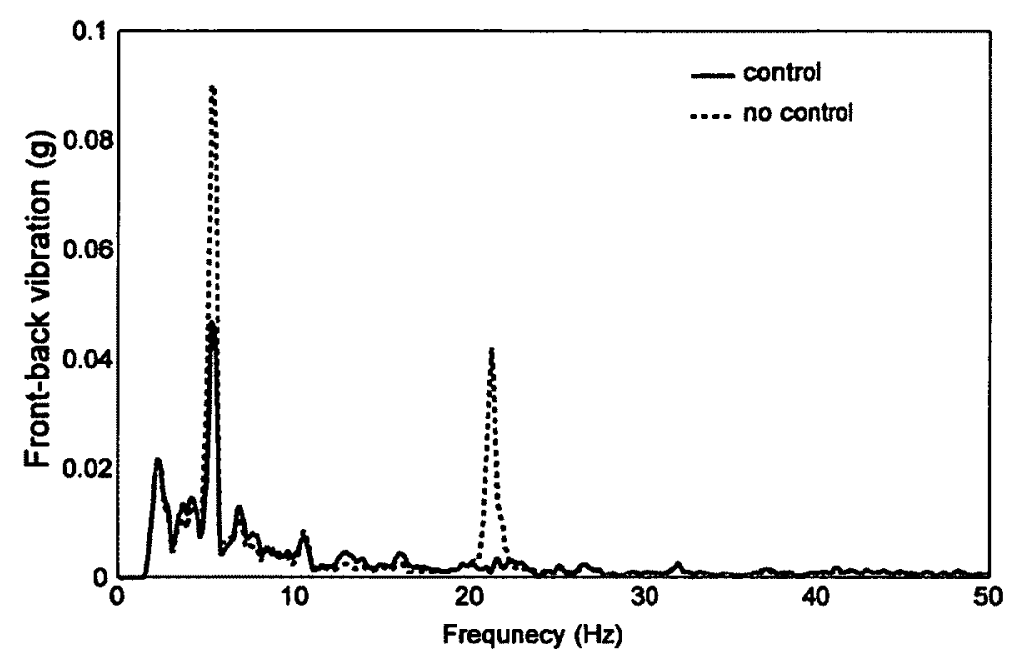

Figure 4.10: Simulation of aircrew helmet vibration in translational flight condition

Simulation results showed that the vibration levels experienced by the aircrew can be reduced through adaptive control of the stacked piezoelectric actuators in all representative flight conditions. The feed-forward controller based on adaptive notch filter with FLMS algorithm was capable of counteracting harmonic vibration under varying excitation conditions. Simulation results demonstrated that the stacked piezoelectric actuators were able to reduce the $1 / \mathrm{rev}$ and $4 / \mathrm{rev}$ peaks in both fore-aft and vertical directions on the aircrew helmet. The suppression to $1 / \mathrm{rev}$ and $4 / \mathrm{rev}$ peaks could result in an overall reduction of the aircrew body vibration by $33.1 \%$ in the fore-aft direction and $28.1 \%$ in the vertical direction.

As expected, high displacement capability was required to counteract the $1 / \mathrm{rev}$, while the requirement for the $4 / \mathrm{rev}$ harmonic was less than $0.1 \mathrm{~mm}$. In addition, high force was required for the suppression of the $4 / \mathrm{rev}$ peak during the cruise flight conditions. Based on the nominal displacement of $0.2 \mathrm{~mm}$ and $1000 \mathrm{~N}$ force capability of the selected stacked piezoelectric actuators, effective suppression of the vibration at the $4 / \mathrm{rev}$ harmonic could be achieved using the proposed adaptive seat mount system. 


\subsection{Feedback Algorithm for Resonance Vibration Control}

Although the feed-forward control algorithm to suppress $\mathrm{N} / \mathrm{rev}$ harmonics was the primary controller, a secondary feedback controller was simultaneously implemented to suppress the resonant modes of the seated mannequin that are excited due to random vibration. In order to simplify the robust feedback controller development for this secondary controller, the actuator plant was assumed to be LTI because the stroke generated by the stacked piezoelectric actuator was small. Based on these assumptions, the Linear Quadratic Gaussian (LQG) regulator was chosen in the present investigation for the feedback control algorithm for its simplicity and inherent robustness. This LQG controller uses the State Space technique for designing optimal dynamic regulators. The chosen LQG regulator had inherent advantages in balancing performance and control effort, as well as the capability to take process and measurement noise into account. The LQG design requires a State Space model of the plant and the State Space realization of the control path $\mathrm{H}(\mathrm{z})$ in the form, with $\boldsymbol{x}_{\boldsymbol{f}}$ as the state vector

$$
\begin{gathered}
\dot{x}_{\beta b}=A_{f b} x_{f b}+B_{\beta b}\left(u_{f b}+w_{f b}\right) \\
y_{f b}=C_{f b} x_{\beta}+D_{f b}\left(u_{b b}+w_{f b}\right)+v_{\beta b}
\end{gathered}
$$

The goal of the LQG regulator was to control the measurement value $y_{f b}$ around zero to ensure reduction in vibration. The plant model also includes the process noise $\boldsymbol{w}_{f b}$ and driven by the control signal $\boldsymbol{u}_{f b}$, while the measurements may include noise $v_{f b}$ from the sensor. The $A_{f b}, B_{f b}, C_{f b}$ and $D_{f b}$ matrices describe the stacked piezoelectric actuator system based on State Space realization. The LQG regulator consists of an optimal statefeedback gain vector $\boldsymbol{K}_{\boldsymbol{f}}$ and a Kalman state estimator. Assuming a linear state feedback gain vector in the form

$$
u_{f b}=-K_{f b} x_{f b}
$$


the optimal controller is formulated by choosing the control input to minimize the quadratic cost function

$$
J=\int_{0}^{\infty}\left\{x_{f b}^{T} Q_{f b} x_{f b}+2 x_{f b}{ }^{T} N_{f b} u_{f b}+u_{f b}^{T} R_{f b} u_{f b}\right\} d t
$$

that forms the LQG control law with the states estimated by a Kalman filter with noise based weighting matrices. The performance of the LQG regulator was measured by a quadratic performance criterion and the weighting matrices $Q_{f b}, N_{f b}, R_{f b}$ defined to tradeoff between performance, noise and control effort. The weighting matrix $\boldsymbol{Q}_{\boldsymbol{f}}$ is referred to as the state weighting, $N_{f b}$ as the noise weighting and $\boldsymbol{R}_{\boldsymbol{f}}$ is the control weighting matrices. These matrices weigh the relative importance of attenuating the response and limiting the control effort while deriving the feedback gain $K_{f b}$ by minimizing the cost function identified in Eq. 4.20. The gain matrix $K_{f b}$ is obtained by solving the algebraic Riccati equation and the design is completed by implementing a Kalman filter, which is an optimal estimator when dealing with Gaussian white noise. The State Space representation of state feedback compensator together with estimated state vector $\hat{\boldsymbol{x}}_{\boldsymbol{f}}$ generated by the Kalman filter is shown in Figure 4.11.

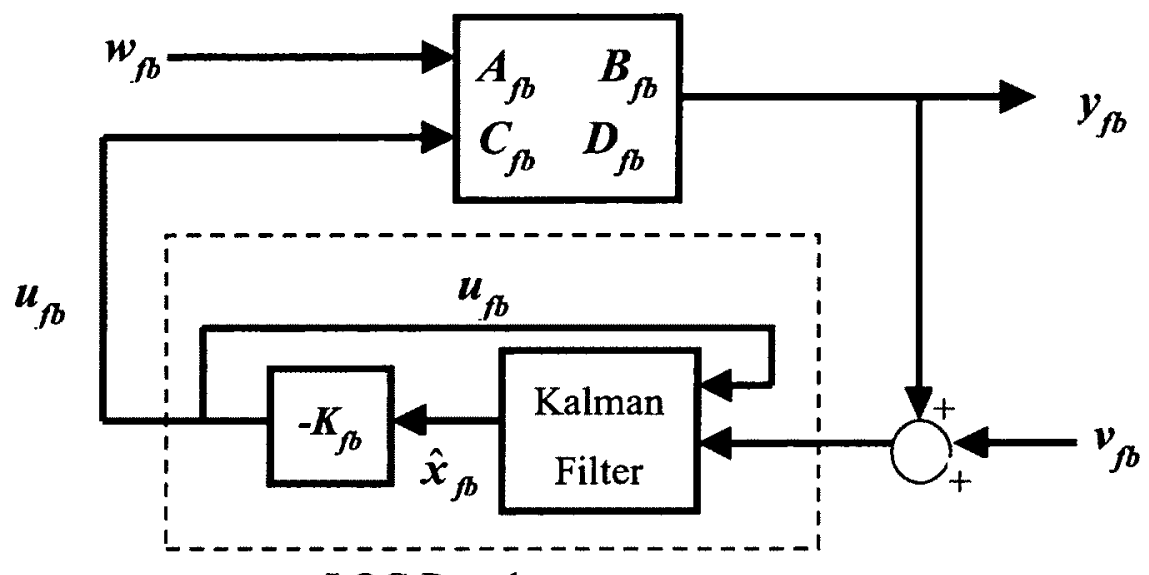

LQG Regulator

Figure 4.11: LQG regulator with Kalman filter 
The MATLAB software was used to compute the feedback gain matrix $\boldsymbol{K}_{\boldsymbol{b}}$ by solving an algebraic Riccati equation. The software provided a flexible platform to derive the gain matrix by balancing the performance and control effort. This LQG feedback controller was also implemented using SIMULINK in parallel to the feedforward control algorithm. Similar to the FLMS based feed-forward control algorithm, the control objective of this LQG feedback control algorithm was also to minimize the vibration of the aircrew helmet. Therefore, the accelerometer that measured the helmet fore-aft vibration was selected as the sensor for the error signal $e(k)$. The details of this well established LQG control law, consisting a full-state regulator in series with a Kalman state estimator, are not discussed in here because it has been published previously by a number of prominent authors [137].

\subsection{Controller Implementation Platform}

The controller was implemented in real-time on the full-scale Bell-412 helicopter seat integrated with the adaptive mount system using both the primary feed-forward control algorithm designed to suppress $\mathrm{N} / \mathrm{rev}$ harmonics as well as the secondary feedback controller to suppress the resonant modes as shown in Figure 4.12.

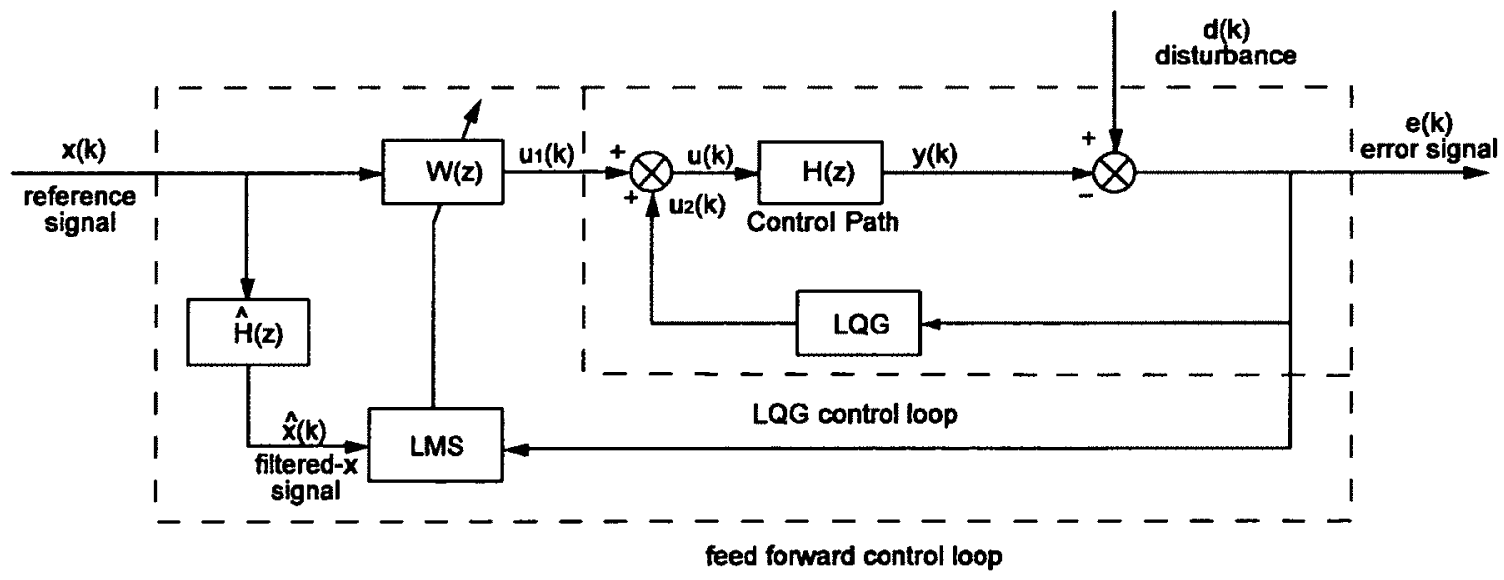

Figure 4.12: Simultaneous implementation of FLMS and LQG algorithms 
Based on the single error signal $\mathrm{e}(\mathrm{k})$, the primary feed-forward FLMS algorithm generated the control input $u_{1}(k)$ while the secondary feedback LQG regulator generated the control output $u_{2}(k)$. This simultaneous implementation generated the total control input by superimposing individual control input signals as:

$$
u(k)=u_{1}(k)+u_{2}(k)
$$

This aggregated control input, $u(k)$, produced corresponding forces from stacked piezoelectric actuators to generate the control output through the control path $\mathrm{H}(\mathrm{z})$ as

$$
y(k)=u(k) * h(k)
$$

to minimize the error signal $\mathrm{e}(\mathrm{k})$ measured by the accelerometer installed on the mannequin helmet in the fore-aft direction.

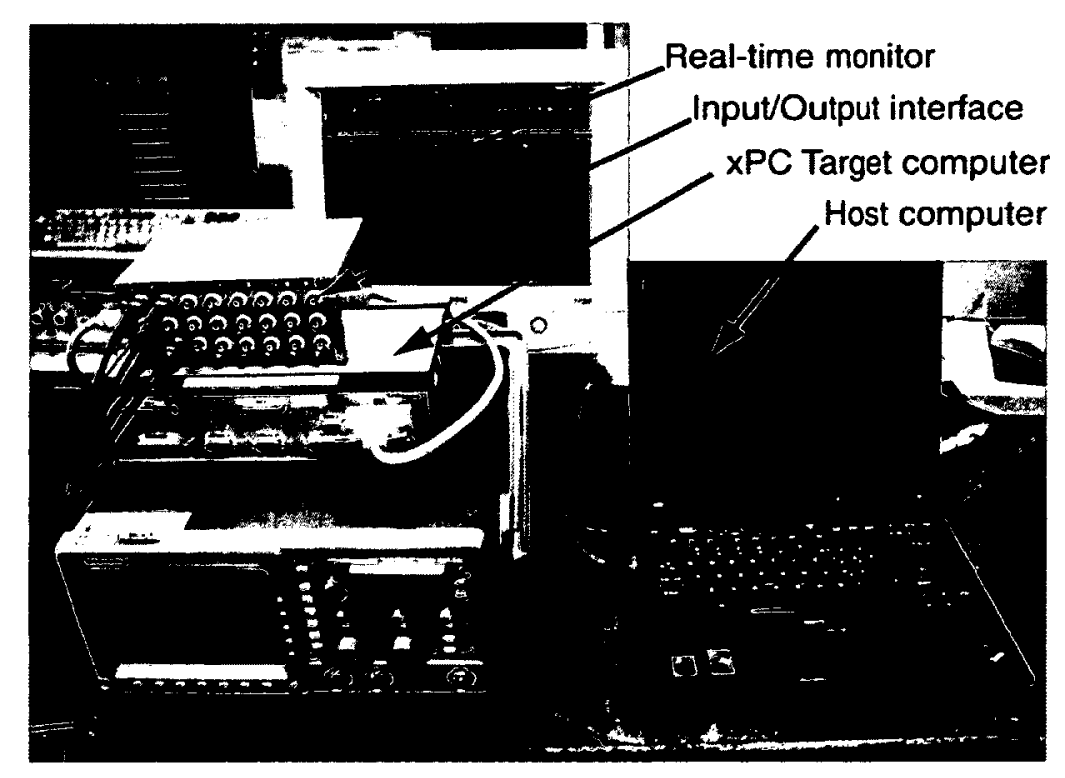

Figure 4.13: xPC Target hardware components 
The control algorithm was implemented in real-time on the full-scale Bell-412 helicopter seat integrated with the adaptive mount system using the MATLAB based XPC Target hardware platform. The hardware components of the real-time controller implementation platform used for closed-loop control tests are shown in Figure 4.13.

This real-time control computer system operated with the SIMULINK software and thus allowed rapid control algorithm prototyping and hardware-in-the-loop experiments for efficient controller implementation as well as optimization of control parameters. A real-time executable code was generated from the SIMULINK block diagram of the controller design on the host computer and then transferred to the xPC Target computer to run in the real-time mode. This real-time control system was capable of handling large state-space models with high state counts and provided the flexibility to configure the control law with user-defined input, output and control parameters for various control algorithm implementations. The xPC Target hardware interfaced seamlessly with sensors and actuators on the full-scale adaptive helicopter seat mount system installed on the shaker table. The available input and output options from the SIMULINK blocks were used to connect to the output of the error sensor, namely, the accelerometer that measured the helmet vibration in the fore-aft direction, as well as the control input to the power amplifier that energized the piezoelectric actuators. The ability of this hardware-in-the-loop control system to use the same software for tests as well as in simulations significantly enhanced the efficiency in control law development as well as implementation. It allowed rapid control algorithm prototyping and control signal verification prior to implementation.

In order to perform closed-loop control experiments, the $10,000 \mathrm{lb}(\mathrm{f})$ UnholtzDickie mechanical shaker test facility was used to provide simulated helicopter floor vibration profiles to the seat frame in the vertical direction. The experimental setup with the block diagram of instrumentation is shown in Figure 4.14. The Bell-412 helicopter seat described in Section 2.2.3 was installed on the shaker in Z-direction excitation and the Sierra Sam type mannequin that represented a $95^{\text {th }}$ percentile male was strapped to the seat in a normal seating posture as described in Section 3.1.2. The vibration profile generated by the shaker was measured using a miniature accelerometer of the type PCB 
$352 \mathrm{C} 10$ with a signal conditioner type B\&K 2635 and was used as the reference signal $x(k)$ for the FLMS controller. A similar accelerometer and a signal conditioner was used to measure the mannequin helmet vibration in the fore-aft direction, which was used as the error signal $\mathrm{e}(\mathrm{k})$. The $\mathrm{xPC}$ Target computer acquired both accelerometer signals and generated the control input in accordance to control logic based on simultaneous implementation of the FLMS algorithm and LQG regulator. This control signal $\mathrm{u}(\mathrm{k})$ was supplied to the LE 150/200-3 high voltage amplifier to energize the Pst 150/14/200 VS20 stacked piezoelectric actuators incorporated on the full-scale Bell-412 helicopter seat as the adaptive mount. The control forces generated by the actuator counteracted the vibration from $\mathrm{N} / \mathrm{rev}$ peaks and resonance modes to suppress vibration at the mannequin helmet locations. The host computer was used to transfer the real-time executable code generated from the SIMULINK block diagram of the controller design and stored the data acquired by the XPC Target computer in the real-time mode.

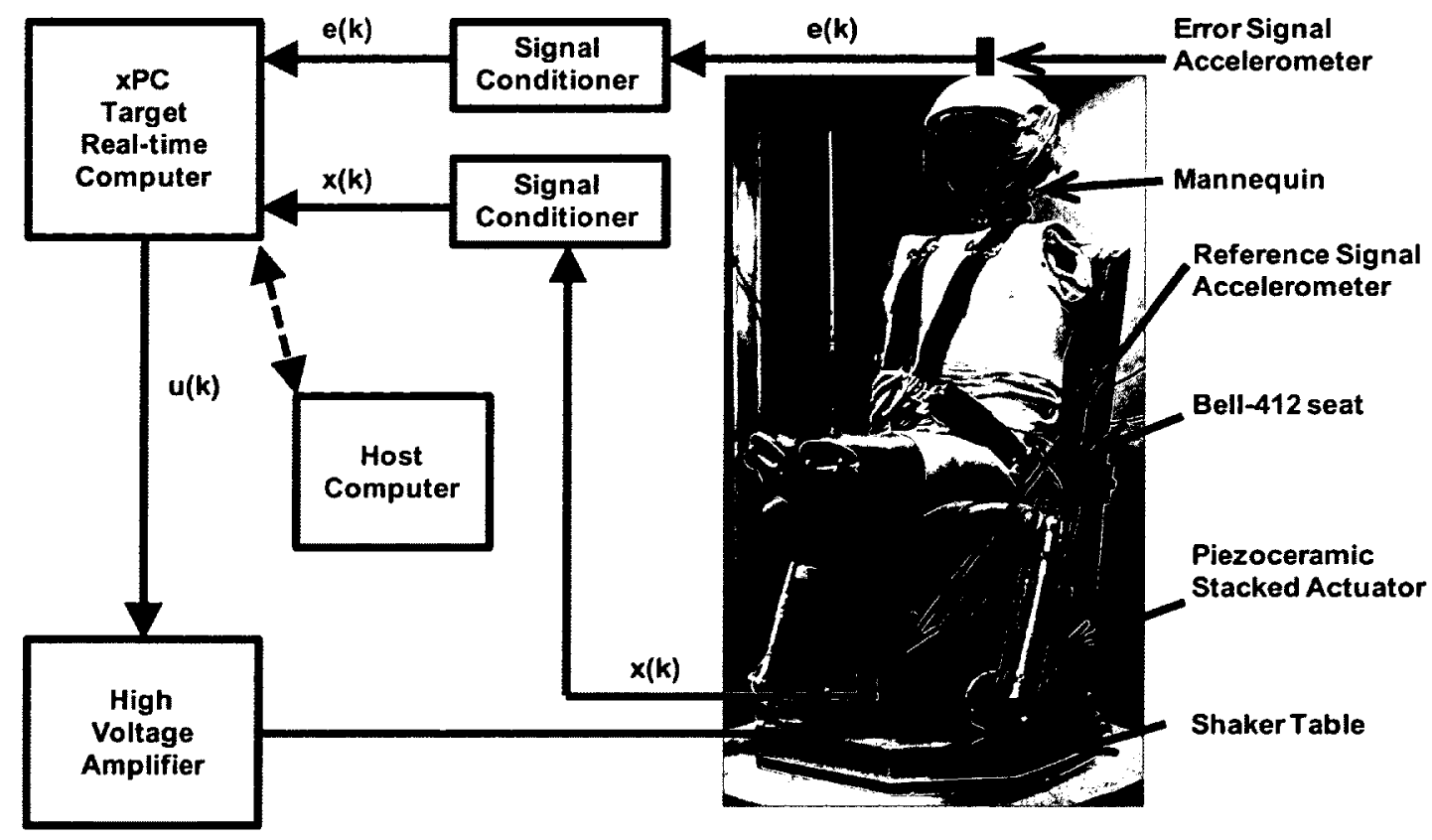

Figure 4.14: Experimental setup with the block diagram of instrumentation 


\subsection{Experimental Performance Verification}

The performance verification of the adaptive seat mount system to mitigate aircrew exposure to vibration was conducted using extensive closed-loop control experiments on the retrofitted full-scale Bell-412 helicopter seat. The objective was to verify the reduction of $\mathrm{N} / \mathrm{rev}$ harmonic peaks as well as resonance modes of the mannequin excited by representative helicopter floor vibration spectra. In the experiments, the "no control" vibration response curves represented the cases that the seat was integrated with the nonactuated piezoelectric stack actuators as passive stiffness struts, while the response curves labeled as "control" represented the cases that the actuators of the adaptive mount were actively controlled by the real-time control algorithm.

\subsubsection{Sinusoidal Vibration Spectrum Test}

Sinusoidal tones were used as the shaker excitation to simulate the $\mathrm{N} / \mathrm{rev}$ harmonic excitations of the Bell-412 helicopter on the aircrew seat frame. Four major harmonic peaks were generated using the mechanical shaker, namely, the $1 / \mathrm{rev}, 2 / \mathrm{rev}, 4 / \mathrm{rev}$ and 8/rev; and the fundamental harmonic frequency was set at $5.4 \mathrm{~Hz}$ to correlate with the rotor speed of the Bell-412 helicopter. The amplitude of each harmonic frequency was set to $0.03 \mathrm{~g}$ and the overall input vibration was $0.045 \mathrm{~g}$-rms. Although the input vibration level represented only $25 \%$ of the floor vibration level at $120 \mathrm{kts}$ cruise flight, the vibration measured on the mannequin helmet was comparable to the level of the pilot helmet location during the flight test. This is attributed to the significant impedance differences between the very rigid shaker table and the relatively flexible helicopter fuselage floor that may have provided additional vibration reduction.

The adaptive seat mount system was able to effectively reduce mannequin vibration levels at all critical measured locations using the real-time controller based on the adaptive notch control algorithm. As shown in Figure 4.15, the four plots described the helmet vibration measured in all three axes as well as the body vibration in the vertical direction. Through adaptive control of the two stacked piezoelectric actuators using the adaptive feed-forward control law, the overall vibration levels at the mannequin 
helmet location and the body locations were reduced effectively and simultaneously. The $2 / \mathrm{rev}$ harmonics in the fore-aft direction of the helmet location was reduced by $68 \%$; the $4 / \mathrm{rev}$ harmonic reduced by $25 \%$ and the overall vibration levels in the fore-aft direction reduced by $52 \%$. Although the vertical and side vibrations at the helmet location were not included as an error signal in the control strategy design, the overall vibration levels were also suppressed simultaneously, with 10\% reduction in the vertical direction and $23 \%$ reduction in the lateral direction. Considering the vibration in all three directions, the equivalent overall vibration level was reduced by $35 \%$ at the mannequin helmet location. Moreover, the vibration level at the mannequin abdomen location was also suppressed by $35 \%$ with the $2 /$ rev harmonic reduced by $49 \%$. Despite the dynamic nonlinearity of the seat cushion and mannequin body, no significant control spillover was observed due to the counteracting force produced by actuators.
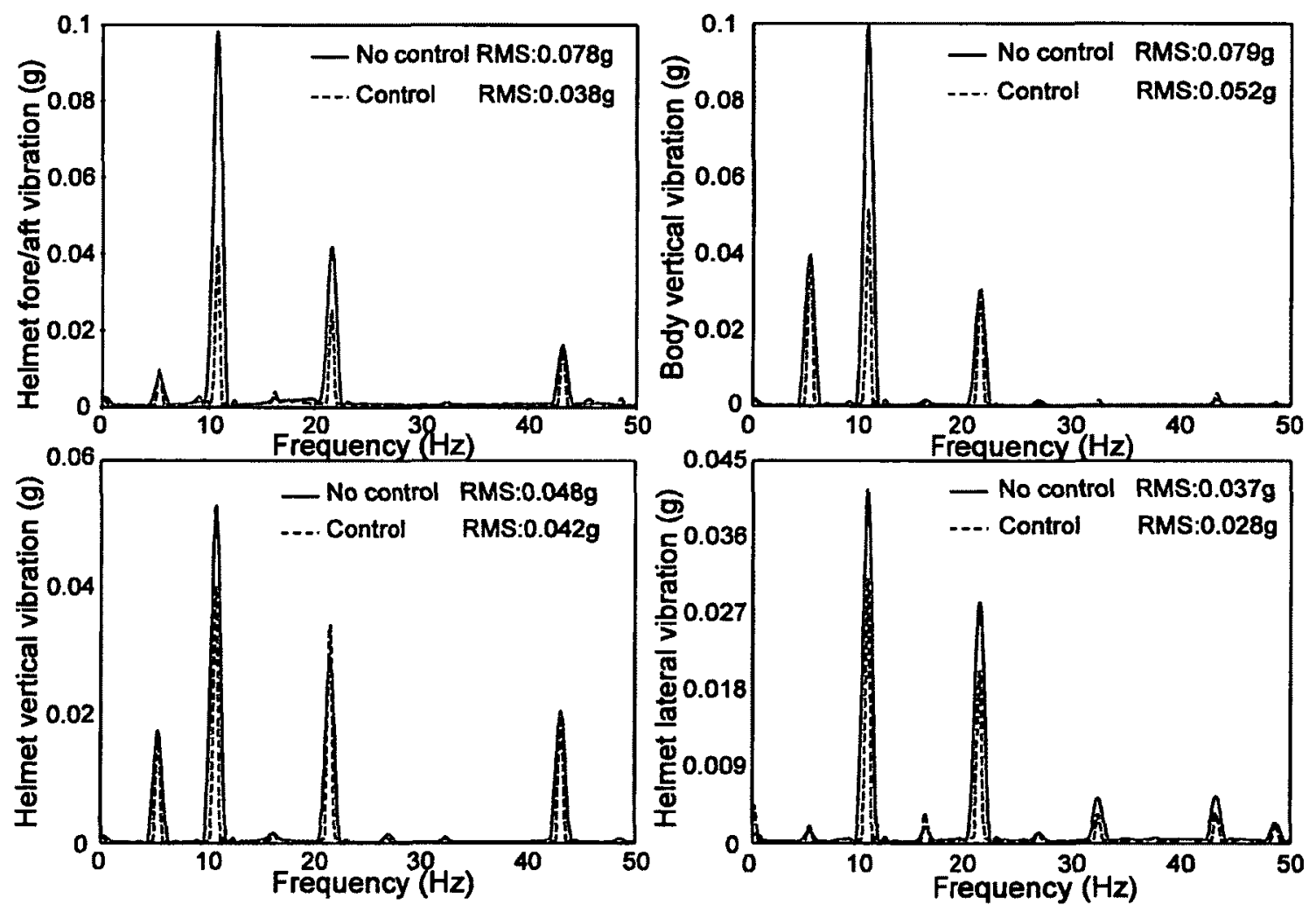

Figure 4.15: Vibration control results under sinusoidal harmonic profile 


\subsubsection{Combined Vibration Profile Test}

Closed-loop experiments performance verification tests were also conducted using combined vibration profiles which included the $\mathrm{N} / \mathrm{rev}$ harmonic tones in addition to the background random spectrum. The $\mathrm{N} / \mathrm{rev}$ harmonic spectrum was kept the same as in the sinusoidal spectrum test discussed previously. The random vibration spectrum covered the frequency range from 4 to $50 \mathrm{~Hz}$ as specified by MIL standard, and the level was kept at $0.02 \mathrm{~g}$-rms. This combined vibration input profile that represented the helicopter floor vibration generated an overall input vibration level of $0.05 \mathrm{~g}$-rms from the shaker to the seat frame in the vertical direction.

The vibration spectrum at the mannequin helmet and body locations with the "no control" condition was dominated by the $2 / \mathrm{rev}$ and $4 / \mathrm{rev}$ peaks as shown in Figure 4.16. As expected, the seat/mannequin modes at $9.7 \mathrm{~Hz}$ and $16.6 \mathrm{~Hz}$ were also excited by the random vibration input. Similarly, the dynamic coupling of the vertical mode at $10.8 \mathrm{~Hz}$ with the $2 / \mathrm{rev}$ harmonic resulted in the highest peak, and the $4 / \mathrm{rev}$ harmonic was also found prominent in the mannequin body vibrations.

The adaptive feed-forward control law achieved simultaneous suppression in major $\mathrm{N} / \mathrm{rev}$ harmonics while the feedback controller reduced the resonant peaks of the mannequin vibrations. Closed-loop control results demonstrated significant vibration reduction at the mannequin helmet location, namely, the $2 / \mathrm{rev}$ harmonic in the fore-aft direction was suppressed by $69 \%$ and the $4 / \mathrm{rev}$ harmonic reduced by $50 \%$. For the resonant peaks, the localized helmet bending mode at $9.7 \mathrm{~Hz}$ was reduced by $44 \%$. However, the mode at $16.6 \mathrm{~Hz}$ was not controlled effectively. As a result, the overall vibration level in the fore-aft direction was suppressed by $39 \%$. Similar to the previous control attempt, both the helmet vertical and side vibrations have been reduced by $7 \%$ and $16 \%$, respectively. No significant control spillover was observed in this closed-loop active control test. The overall equivalent vibration reduction at the helmet location was $26 \%$ when considering the vibrations in three directions. Notably, vibration at the mannequin abdomen location was reduced by $33 \%$. The $2 / \mathrm{rev}$ and $4 / \mathrm{rev}$ harmonics at the abdomen location was suppressed by $50 \%$ and $26 \%$, respectively. In addition to forced 
excitation peaks, the resonance vibration modes at $9.7 \mathrm{~Hz}$ and $16.6 \mathrm{~Hz}$ at the abdomen location were suppressed by $53 \%$ and $23 \%$, respectively.
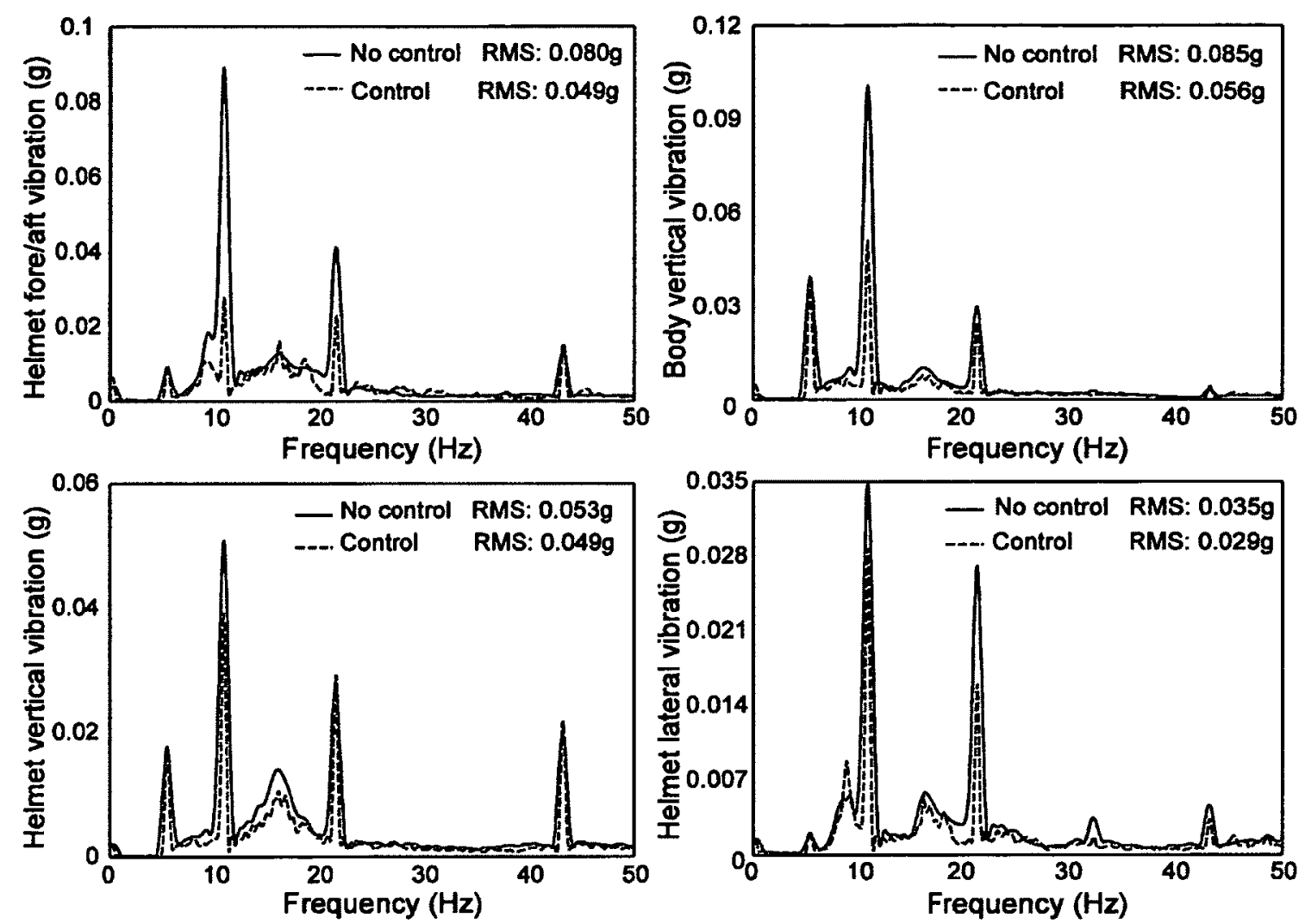

Figure 4.16: Vibration control results under combined vibration profile

\subsubsection{Discussion of the Adaptive Mount Performance}

Based on extensive closed-loop control experiments, significant vibration reduction at the mannequin helmet location was demonstrated through adaptive real-time control of the two stacked piezoelectric actuators integrated on the full-scale Bell-412 helicopter seat. The adaptive feed-forward control law achieved simultaneous suppression in major N/rev harmonics while the feedback controller reduced the resonant peaks of the mannequin vibrations. The control results demonstrated significant vibration reduction at the mannequin helmet location at $2 / \mathrm{rev}$ as well as $4 / \mathrm{rev}$ harmonics to the rotor frequency. In addition to the reduction in forced excitation peaks, the resonance vibration modes were 
also suppressed effectively by the adaptive seat mount system implemented on the fullscale Bell-412 helicopter seat.

However, the closed-loop control results showed no appreciable reduction to the $1 /$ rev harmonics excitation of the mannequin helmet. Several factors contributed to this effect. First, integration of the piezoelectric actuators as structural struts increased the structural stiffness of the seat to move the bending vibration mode at $5.4 \mathrm{~Hz}$ out of the low frequency regime to reduce the response generated by the $1 /$ rev excitation frequency. Therefore, the modified full-scale Bell-412 seat integrated with the adaptive mount system was not susceptible for $1 /$ rev excitation similar to the original seat structure. Secondly, the objective of feed-forward control law was to minimize the root mean square value of the error signal, which was selected as the fore-aft acceleration at the helmet location. This would imply more control weights are applied to the $2 / \mathrm{rev}$ and 4/rev harmonics as the amplitudes were significantly higher when compared to $1 / \mathrm{rev}$. Thirdly, since the $1 / \mathrm{rev}$ was lower in frequency, it required a larger stroke from the actuators to achieve effective vibration suppression. However, the currently used stack piezoelectric actuators may not have provided sufficient stroke to counteract the low frequency vibration. Vibration reduction performance at $1 /$ rev may be improved by using stroke improved actuators for the adaptive seat mount design.

In this investigation, it is important to note that the vibration input from the shaker was limited to $25 \%$ of the nominal Bell- 412 helicopter floor vibration levels at $120 \mathrm{kts}$. The control authority of stacked piezoelectric actuators was also intentionally limited to $50 \%$ of its full capacity to protect the delicate and expensive actuator hardware. However, the proof-of-concept adaptive seat mount system has shown very promising results that verifies reduction in the mannequin body vibration levels without significant modification to the helicopter structure.

\subsection{Summary and Conclusions}

In summary, a novel adaptive helicopter seat mount design, which included two stacked piezoelectric actuators to counteract the forced excitation, was developed to provide 
effective aircrew vibration suppression to reduce adverse health effects. This unique implementation of an active vibration control system for the helicopter seat vibration reduction application is one of the main contributions of this thesis. Extensive dynamic simulations were used in the design process to determine the actuators specifications and develop the feed-forward control algorithm with an adaptive notch controller to control multiple rotor harmonics in a broad frequency band.

Another important contribution of this research is the successful implementation of a proof-of-concept adaptive helicopter seat mount retrofitted on a full-scale Bell-412 aircrew seat to demonstrate vibration suppression performance. A real-time control computer was used to implement the Filtered-x Least Mean Square feed-forward control algorithm based on adaptive notch filters to suppress forced vibration frequencies as well as a robust feedback control law simultaneously to suppress resonance modes. Closedloop control experiments performed using shaker tests verified that the proposed adaptive seat mount methodology is able to provide significant reduction in $\mathrm{N} / \mathrm{rev}$ harmonic peaks as well as reduce the resonant modes under representative helicopter vibration spectra. This investigation verified that the adaptive seat mount based on active control technology is a viable solution for the helicopter seat vibration control application. 


\section{Chapter 5}

\section{Investigation of Energy Absorbing Seat Cushion Materials}

This chapter investigates the use of novel energy absorbing seat cushion materials to reduce the vibration transmitted to the aircrew in order to mitigate the associated health issues. Although the modifications to the seat structure with active or passive struts provide significantly high vibration suppression performance for the aircrew, the implementation of these solutions in operational aircraft require an expensive and long airworthiness certification effort as indicated by literature surveys and discussions with technical personnel. Further investigations suggested that replacing the cushion material on a helicopter seat is a low cost solution with less certification effort, even though it provided relatively lower vibration suppression performance compared to modifications to the seat structure. Therefore, energy absorbing seat cushion materials were evaluated to mitigate vibration in Bell-412 helicopter seats, particularly to reduce the vibration amplification measured 1/rev peak. Material characterization tests using the mechanical shaker facility and flight tests on the NRC Bell-412 helicopter were used to validate the novel cushion materials to mitigate the vibration transmitted to the aircrew.

\subsection{Helicopter Seat Cushion Design}

Helicopter aircrews, particularly of military rotorcraft, are often required to spend long periods of time in their seats while performing their primary duties. Military helicopter 
pilots are known to routinely spend up to $6+$ hours in their seats performing operations that include pre-flight, flight, and post-flight tasks. Therefore, the seat cushion design directly impacts the crew comfort. As the primary contact point for the transmission vibration or shock forces, helicopter seat cushions are designed to dissipate some energy prior to transmitting these forces to the human body. Therefore, proper selection of the cushion material with optimized impedance properties can significantly reduce the aircrew exposure to vibration.

\subsubsection{Applicable Cushion Design Requirements}

Classical passive vibration isolation techniques based on transmissibility plot indicate that the resonance frequency of the mechanical system is required to be 4 to 5 times lower than the lowest harmonic frequency of the excitation input to achieve effective isolation [138]. Such a design requires the Bell-412 helicopter seat cushion to have an impractically low stiffness that generates a large displacement to isolate the $1 /$ rev rotor excitation frequency at $5.4 \mathrm{~Hz}$. This is stipulated in MIL-STD-81771 A, which states that the cushion design must avoid introducing dynamic overshoot of the system to guarantee stability while ensuring comfort and durability [139]. This helicopter seat design standard also requires that the cushion is not displaced or separated from the seat during aircraft manoeuvres, crashes, or occupant motion. For vibration isolation, the MIL-STD85510 stipulates that the "occupied and unoccupied seat shall be free of resonance within the frequency range of the aircraft in which it will be used and no amplification shall occur" [9]. These design requirements must be considered in selecting novel cushion materials for the Bell-412 helicopter seat application as adhering to these standards is essential to gain airworthiness certification for any potential seat cushion design.

\subsubsection{Proposed Cushion Modification Approach}

As discussed in the flight tests in Chapter 2, the level of vibration of the aircrew is influenced by several factors, including the flight condition, aircrew weight, posture, and dynamic characteristics of the seat, amongst many other factors. Modification to the seat 
cushion was considered as a low-cost solution that could be implemented as near-term solution to mitigate vibration induced health issues in helicopter aircrew. Conventional cushions typically increase the transmission of vertical vibration at low frequencies in the vicinity of the primary human whole-body resonance $(4-8 \mathrm{~Hz})$ while attenuating the transmission of vibration at higher frequencies [140]. This amplification of low frequency vibration was evident from the aircrew vibration measured during the Bell-412 helicopter flight test. However, the Z-direction vibration transmission path spectra for both the pilot and co-pilot shown in Figure 2.11 indicate that the original in-service cushion used in the Bell-412 helicopter seat achieved significant reduction in high frequency vibration, although it amplified the $1 /$ rev vibration peak.

Therefore, the proposed approach was to use the properties of the existing cushion for high frequency vibration reduction while integrating a novel energy absorbing cushion material with appropriate impedance properties to reduce the vibration amplification at $1 / \mathrm{rev}$ peak. In an attempt to evaluate novel seat cushion materials to dampen $5.4 \mathrm{~Hz}$ vibration transmitted through the seat structure, various commercially available cushion materials were preliminarily tested in the laboratory using the shaker tests to characterize the material. Thereafter, the performance of selected cushion materials to improve vibration aircrew exposure was verified using the NRC Bell-412 helicopter under typical flight conditions. It is important to note that this effort was not to develop new cushion materials but to investigate the use of commercially available novel cushion materials as a low cost solution for the helicopter seat application.

\subsection{Evaluated Novel Cushion Materials}

Based on literature search, a database of novel cushion materials used for similar vibration reduction applications was established. Priority was given to identify novel energy absorbing materials that have been used for other vibration reduction applications that can be used for the helicopter seat application. In addition, consideration was given to materials that have been used to dampen low frequency vibration in applications such as insoles in shoes. This is because the frequency of the walking rhythm is 
approximately $2 \mathrm{~Hz}$, which is the same frequency range as the $1 / \mathrm{rev}$ rotor harmonic. Consideration was also given for cushion materials that have been used in the aircraft industry to minimize the certification process in retrofitting the Bell-412 helicopter seats with the selected novel cushion materials. Based on the above criterion and energy absorbing performance, engineered cushion known as Hybrid Air Cushioning System and viscoelastic polyurethane material were chosen as two of the primary cushion materials to be investigated to dampen the $1 / \mathrm{rev}$ vibration peak in the Bell-412 helicopter seats. Both of these cushion materials were chosen for this study not only due to their superior vibration suppression performance but also due to the fact that they were commercially available in a variety of forms and therefore facilitate the development of a low cost solution for the helicopter seat application.

\subsubsection{Hybrid Air Cushioning System - Mitigator}

An engineered cushion that incorporates an unique Hybrid Air Cushioning System (HACS) known as Mitigator has been used recently in impact protection seating structures and blast resistant flooring systems [141,142]. This HACS is designed to transfer the vertical impact energy through a series of internal bleed ports and passages to absorb energy [143,144]. The picture of the Mitigator cushioning structure in Figure 5.1 shows the hexagonal cells with interconnecting vents that forces the airflow in the cushion to choke. It is this choked airflow that bleeds the energy and redirects much of the energy laterally instead of through the thickness of the cushion.

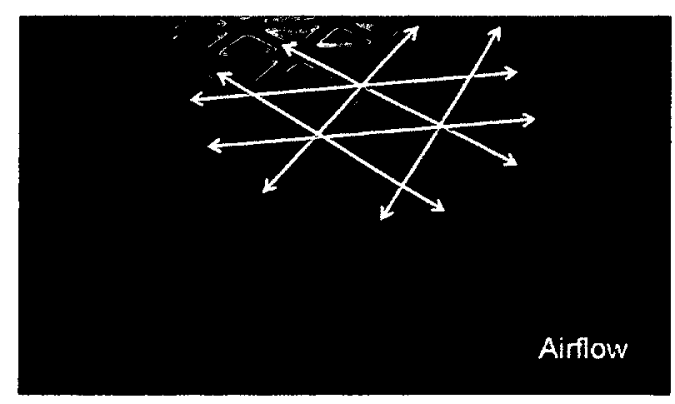

Figure 5.1: Mitigator cushioning structure with interconnected vents 
The 1" thick Mitigator cushion pads were manufactured by Sea Systems Inc. in Clarksville, VA, USA with three different material variations to evaluate vibration suppression performance, namely, urethane, monoprene, and uniprene. Relevant properties of these material types used in the construction of Mitigator cushions provided by the manufacturer are listed in Table 5.1. In the table, durometer is a measure of hardness used for polymers and a higher durometer value indicates increased resistance to permanent indentation.

Table 5.1: Material properties of Mitigator cushions

\begin{tabular}{|c|c|c|c|c|c|}
\hline $\begin{array}{c}\text { Material } \\
\text { Type }\end{array}$ & $\begin{array}{c}\text { Tensile } \\
\text { Strength (psi) }\end{array}$ & $\begin{array}{c}\text { Durometer } \\
(-)\end{array}$ & $\begin{array}{c}\text { Specific } \\
\text { Gravity (-) }\end{array}$ & $\begin{array}{c}\text { Tensile } \\
\text { Elongation (\%) }\end{array}$ & $\begin{array}{c}\text { Young's } \\
\text { Modulus (psi) }\end{array}$ \\
\hline Mitigator - Urethane & 595 & 31 & 1.11 & 975 & 102 \\
\hline Mitigator - Monoprene & 700 & 42 & 1.22 & 700 & 70 \\
\hline Mitigator - Uniprene & 330 & 25 & 0.91 & 375 & 85 \\
\hline
\end{tabular}

Although the Mitigator cushion has not been investigated previously for helicopter seat vibration suppression application, it has been used by the US Navy on the Cobra AH-1W helicopter seat to improve its crash safety. Therefore, the urethane material used in the Mitigator cushions have passed the flammability tests in accordance to the FAR 25.853 aviation standard, as required for aerospace application of these novel energy absorbing cushions.

\subsubsection{Viscoelastic Polyurethane - Sorbothane}

Viscoelastic polyurethane commercially known as Sorbothane is another novel cushion material chosen to be investigated to dampen the $1 / \mathrm{rev}$ vibration in the Bell-412 helicopter seats. Sorbothane is a highly-damped, viscoelastic thermoset material based on polyurethane used for shock and vibration suppression applications [145]. This soft polyurethane cushion material is produced by Sorbothane Inc. in Kent, OH, USA. Although the supplier only provided Sorbothane material pads with thickness of 1" and 0.25 " for this study, the thickness of 0.5 " was configured by adding two layers of 0.25 " 
pads. This material exhibits hysteresis characteristics that absorb the mechanical energy through molecular friction that generates heat during deformation. The heat generated through this mechanism is dissipated efficiently through the material to avoid significant rise in the temperature. The Sorbothane material is currently being used extensively in manufacturing anti-vibration gloves, palm pads, tool wraps, and shoe insoles to minimize the effects of impact energy. Although this material has been used in seat cushion, it has not been investigated for helicopter seat application. The relevant properties of three variations of Sorbothane material provided by the manufacturer are listed in Table 5.2.

Table 5.2: Sorbothane material properties

\begin{tabular}{|c|c|c|c|c|c|}
\hline $\begin{array}{c}\text { Material } \\
\text { Type }\end{array}$ & $\begin{array}{c}\text { Tensile } \\
\text { Strength (psi) }\end{array}$ & $\begin{array}{c}\text { Durometer } \\
\mathbf{( - )}\end{array}$ & $\begin{array}{c}\text { Specific } \\
\text { Gravity (-) }\end{array}$ & $\begin{array}{c}\text { Tensile } \\
\text { Elongation (\%) }\end{array}$ & $\begin{array}{c}\text { Young's } \\
\text { Modulus (psi) }\end{array}$ \\
\hline Sorbothane-30 & 83 & 30 & 1.37 & 582 & 90 \\
\hline Sorbothane-50 & 123 & 50 & 1.36 & 568 & 105 \\
\hline Sorbothane-70 & 206 & 70 & 1.36 & 399 & 120 \\
\hline
\end{tabular}

\subsubsection{Material Characterization using Shaker Tests}

Accurate measurement of these parameters was critical to evaluate the performance of these novel materials to reduce vibration in helicopter aircrew. In addition, it was important to verify the damping and stiffness properties provided by the material suppliers to use them with confidence in the biodynamic model discussed in Section 3.3. Therefore, inverse characterization was performed to determine the relevant properties of the Mitigator and Sorbothane cushion materials using the mechanical shaker [146]. This was achieved by experimentally measuring the vibration response of the cushion material under the influence of random excitation input to represent the 1 DOF mass-spring model. The cushion material was placed on the vibration table driven by a programmable vibration controller set up to generate random vibrations within the frequency range of 3 $\mathrm{Hz}$ to $100 \mathrm{~Hz}$. A known mass, $M$, of $26 \mathrm{~kg}$ was glued to the cushion material to generate both tension and compression by moving the mass freely in the vertical direction. In addition, one third of the mass of the cushion material, $m_{c}$, was also included in the 
effective total mass for the cushion based on published data on oscillating springs [147]. One accelerometer was placed on the shaker table to measure the excitation input and another accelerometer was placed on the moving mass to measure the response output.
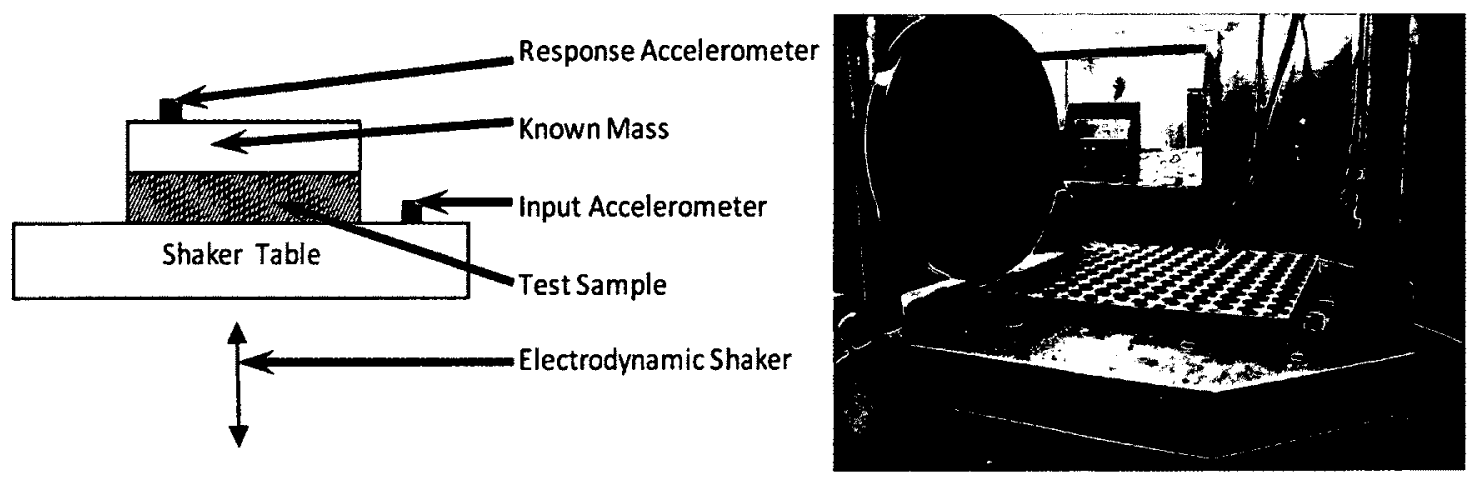

Figure 5.2: Shaker test setup to extract cushion material properties

The LMS Tes.Lab software was used to generate the Transfer Function $H(s)$ using the experimentally measured excitation and response acceleration signals in accordance to the $H_{1}$ formulation in Eq. 3.2 based on the 1 DOF mass-spring model described by

$$
m \ddot{x}(t)+c \dot{x}(t)+k x(t)=f(t)
$$

where the mass, damping coefficient and stiffness are denoted by $m, c$, and $k$, respectively. Transforming Eq. 5.1 to the Laplace domain while assuming zero initial conditions yields

$$
Z(s) X(s)=F(s)
$$

with $Z(s)$ the dynamic stiffness

$$
Z(s)=m s^{2}+c s+k
$$

Assuming the transfer function $H(s)$ between displacement and force as 


$$
X(s)=H(s) F(s)
$$

yields the transfer function of the 1 DOF mass-spring model as

$$
H(s)=\frac{1}{m s^{2}+c s+k}
$$

The roots of the denominator of the transfer function are the poles of the above system. In mechanical structures such as the cushion, the damping coefficient $c$ is usually very small resulting in a complex conjugate pole pair shown below

$$
\lambda=-\sigma \pm i \omega_{d}
$$

with the damped natural frequency

$$
f_{d}=\omega_{d} / 2 \pi
$$

the undamped natural frequency

$$
f_{n}=\omega_{n} / 2 \pi
$$

where

$$
\omega_{n}=|\lambda|=\sqrt{k / m}
$$

and the damping ratio

$$
\xi=\sigma /|\lambda|=c / 2 m \omega_{n}
$$




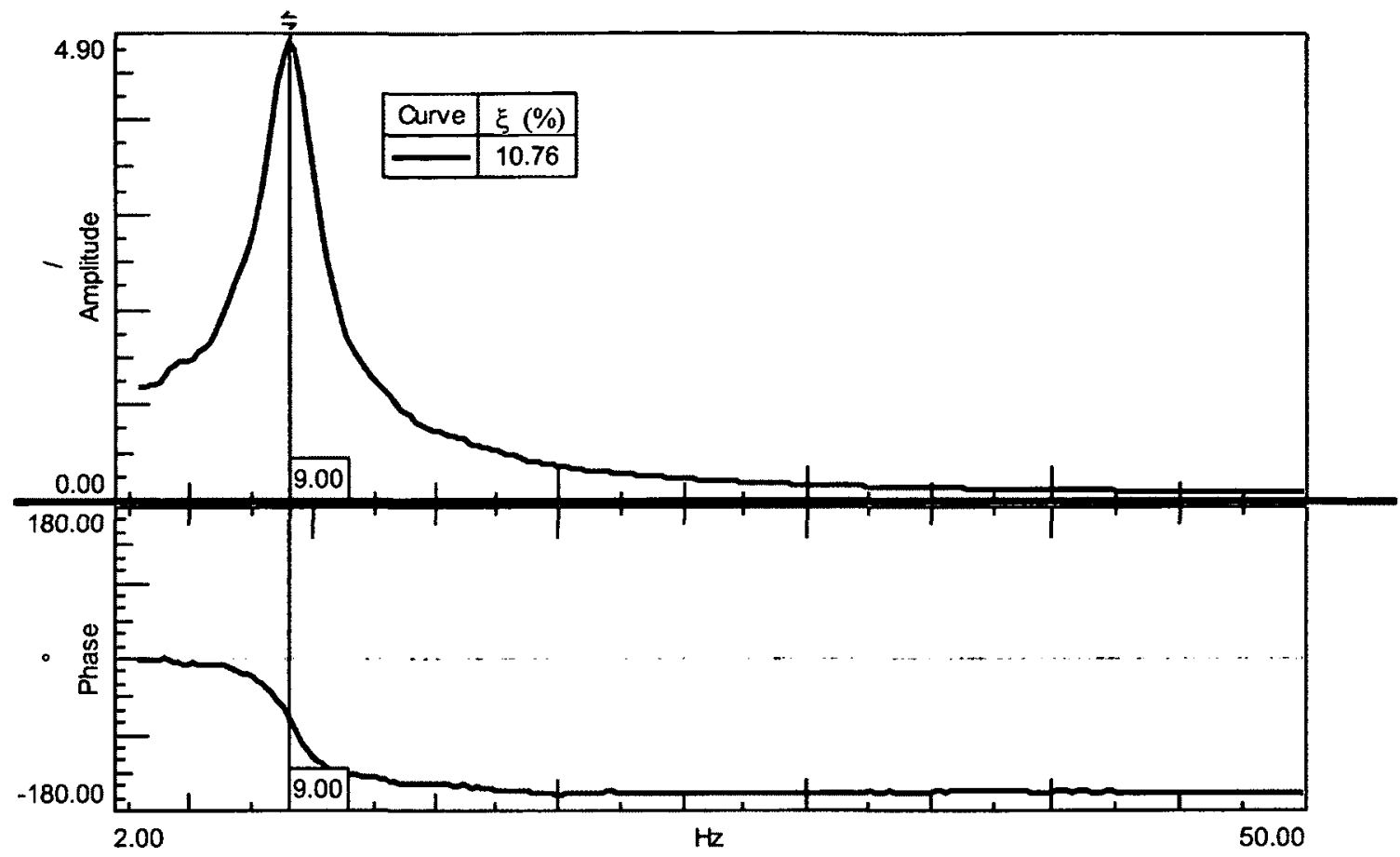

Figure 5.3: Transfer Function of the original cushion $\left(\xi=0.11\right.$ and $\left.f_{d}=9.00 \mathrm{~Hz}\right)$

Using the LMS Tes.Lab analysis software, damped natural frequency, $f_{d}$, and the associated damping ratio, $\xi$, of the 1 DOF mass-spring model was extracted through the curve fitting technique of the transfer function shown in Figure 5.3. Therefore, the undamped natural frequency, $f_{n}$, of the mass-spring system was derived as follows:

$$
f_{n}=\frac{f_{d}}{\sqrt{1-\xi^{2}}}
$$

The Eq. 5.9 was used to calculate the stiffness of the cushion, $k_{c}$ :

$$
k_{C}=\left(2 \pi f_{n}\right)^{2} m_{t o t}
$$


where, the total mass $m_{t o t}=M+1 / 3 m_{c}$ is used for the calculation. The viscous damping, $c_{C}$, for the cushion material was calculated as

$$
c_{C}=2 \xi \sqrt{k_{C} m_{C}}
$$

Based on shaker tests, the material properties shown in Table 5.3 were extracted for various novel cushions and their combinations with the original in-service cushion.

Table 5.3: Extracted material properties of cushion material

\begin{tabular}{|c|c|c|c|c|c|}
\hline $\begin{array}{c}\text { Material } \\
\text { Combination }\end{array}$ & $\begin{array}{c}f_{d} \\
(\mathbf{H z})\end{array}$ & $\begin{array}{c}\xi \\
(-)\end{array}$ & $\begin{array}{c}\mathbf{m}_{\mathbf{c}} \\
(\mathbf{k g})\end{array}$ & $\begin{array}{c}\mathbf{k}_{\mathbf{c}} \\
(\mathbf{P a} \mathbf{~ m})\end{array}$ & $\begin{array}{c}\mathbf{c}_{\mathbf{c}} \\
(-)\end{array}$ \\
\hline Urethane & 35.29 & 0.10 & 3.50 & 1278310 & 1140 \\
\hline Monoprene & 27.46 & 0.04 & 3.00 & 773990 & 341 \\
\hline Uniprene & 34.09 & 0.06 & 3.50 & 1192850 & 663 \\
\hline Sorbothane - 0.25" & 41.52 & 0.11 & 1.50 & 2041425 & 1665 \\
\hline Sorbothane -1.00" & 46.13 & 0.12 & 7.00 & 2268250 & 1850 \\
\hline Original cushion & 9.00 & 0.11 & 5.00 & 76260 & 322 \\
\hline Urethane + Original & 8.19 & 0.12 & 8.50 & 68850 & 325 \\
\hline Monoprene+ Original & 8.19 & 0.10 & 8.00 & 66880 & 272 \\
\hline Uniprene+ Original & 8.29 & 0.11 & 8.30 & 67650 & 281 \\
\hline Sorbothane 0.25" + Original & 8.79 & 0.10 & 7.60 & 77400 & 325 \\
\hline Sorbothane 0.5"+ Original & 8.79 & 0.11 & 8.00 & 79300 & 328 \\
\hline Sorbothane 1.0"+ Original & 8.79 & 0.12 & 12.00 & 82360 & 345 \\
\hline
\end{tabular}

These extracted material properties were verified by comparing with the data provided by the manufacturer of Sorbothane, the only supplier that provided complete set of material properties. For example, the manufacturer reported $\mathrm{k}_{\mathrm{C}}$ of 2,268,000 $\mathrm{Pa}-\mathrm{m}$ and $c_{c}$ of 1850 for Sorbothane material with 1 " thickness and these values resulted in experimental error of $2.5 \%$ and $0 \%$, respectively. This comparison provided the confidence in extracted material properties of the remaining cushion materials. 
Based on experimentally extracted properties of cushion materials, the MATLAB software was used to determine the composite parameters of two cushion materials mechanically integrated in a series configuration. The experimentally acquired FRF for the original cushion integrated with 1 " thick Sorbothane pad compared with the dynamic model developed from extracted parameters of individual cushion components is shown in Figure 5.4. This comparison proves that the dynamic model developed in this study was able to represent the response of combined cushion effectively.

The extracted parameters of these cushion materials were used in the biodynamic model to evaluate the effectiveness of various seat cushion material combinations to mitigate vibration on helicopter aircrew. The dynamic simulation results showed only marginal improvement in aircrew helmet vibration reduction due to integration of cushion combinations. This was due to the simplicity of the model that could not accommodate the effects of highly non-linear cushion materials. Therefore, it was necessary to investigate the performance of the novel energy absorbing cushions through shaker tests as well as flight tests by integrating them with the original cushion of the full-scale Bell-412 helicopter seat.

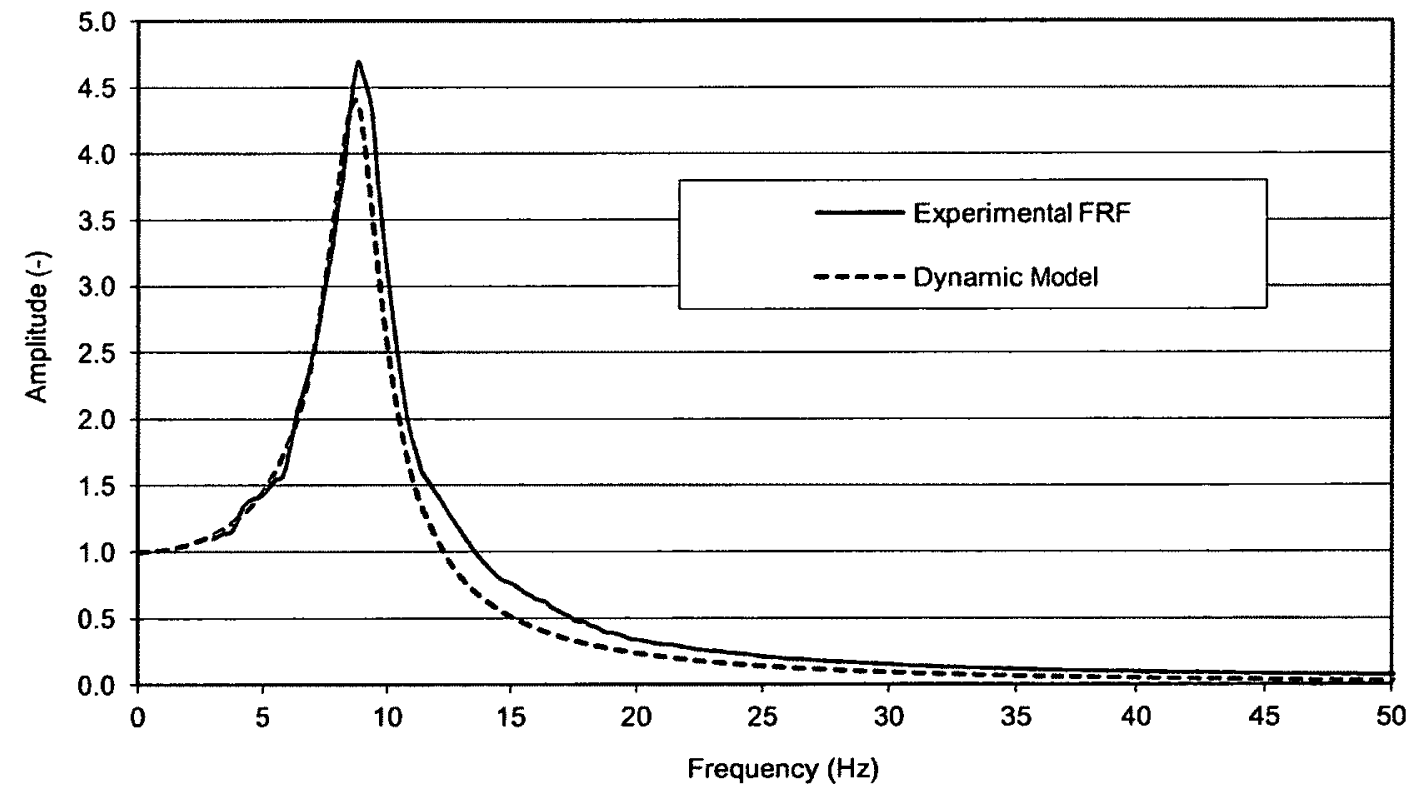

Figure 5.4: Experimental FRF and dynamic model of combined cushion 


\subsection{Mechanical Shaker Tests to Extract Material Properties}

The mechanical shaker tests using the large electrodynamic shaker as described in Section 3.1.2 was used to evaluate the effect of novel cushions in the laboratory to reduce aircrew vibration. This task was accomplished by performing base excited vibration tests on the full-scale Bell-412 helicopter seat by integrating combination of novel cushion materials. The shaker was excited using a random signal generated in the $3 \mathrm{~Hz}$ to $50 \mathrm{~Hz}$ band with an amplitude of $0.25 \mathrm{~g}$-rms to measure the vibration transmissibility variation due to various cushion combinations. The accelerometer placed on the shaker table in the Z-direction was used as the reference and the response of the $95^{\text {th }}$ percentile male mannequin was measured using accelerometers installed on the bottom seat cushion interface in both $\mathrm{Z}$ and $\mathrm{X}$ directions. Only the accelerometer data at the cushion interface location was used as the primary measurement to assess the effect of the whole body vibration exposure on aircrew as per the ISO 2631 standard discussed in Section 2.3.3. The acceleration measured in RMS was used to evaluate the overall magnitude of vibration across the entire frequency range of the vibration input.

Table 5.4: Percentage of reduction for each cushion combination based on g-rms

\begin{tabular}{|c|c|c|c|}
\hline Cushion Combination & Z-Direction & X-Direction & Overall \\
\hline Urethane + Original & $6.0 \%$ & $8.8 \%$ & $7.4 \%$ \\
\hline Monoprene+ Original & $-0.9 \%$ & $-2.5 \%$ & $-1.7 \%$ \\
\hline Uniprene+ Original & $3.4 \%$ & $0.0 \%$ & $1.7 \%$ \\
\hline Sorbothane 0.25"+ Original ' & $6.8 \%$ & $5.0 \%$ & $5.9 \%$ \\
\hline Sorbothane 0.5"+ Original & $5.1 \%$ & $7.5 \%$ & $6.3 \%$ \\
\hline Sorbothane 1.0"+ Original & $6.0 \%$ & $5.0 \%$ & $5.5 \%$ \\
\hline
\end{tabular}

Compared to the original in-service cushion used in the Bell-412 helicopter, the percentage of reduction in vibration measured in both $\mathrm{Z}$ and $\mathrm{X}$ directions on the interface for selected novel cushion combinations are shown in Table 5.4. The results show that combining the original cushion with novel energy absorbing cushion materials, except for the Mitigator cushion manufactured with monoprene material, provided appreciable reduction in vibration transmitted to the aircrew. Although the reduction is relatively 
small, it was achieved without significant structural modifications to the seat. The original in-service cushion with the urethane cushion showed the highest overall reduction of $7.5 \%$, followed by the Sorbothane material of 0.5 " thickness which showed an overall reduction of $6.3 \%$ within the frequency range from $3 \mathrm{~Hz}$ to $50 \mathrm{~Hz}$. In contrast, integrating the original cushion with the Mitigator cushion manufactured with monoprene material showed an increase in vibration in both the $\mathrm{X}$ and $\mathrm{Z}$ directions.

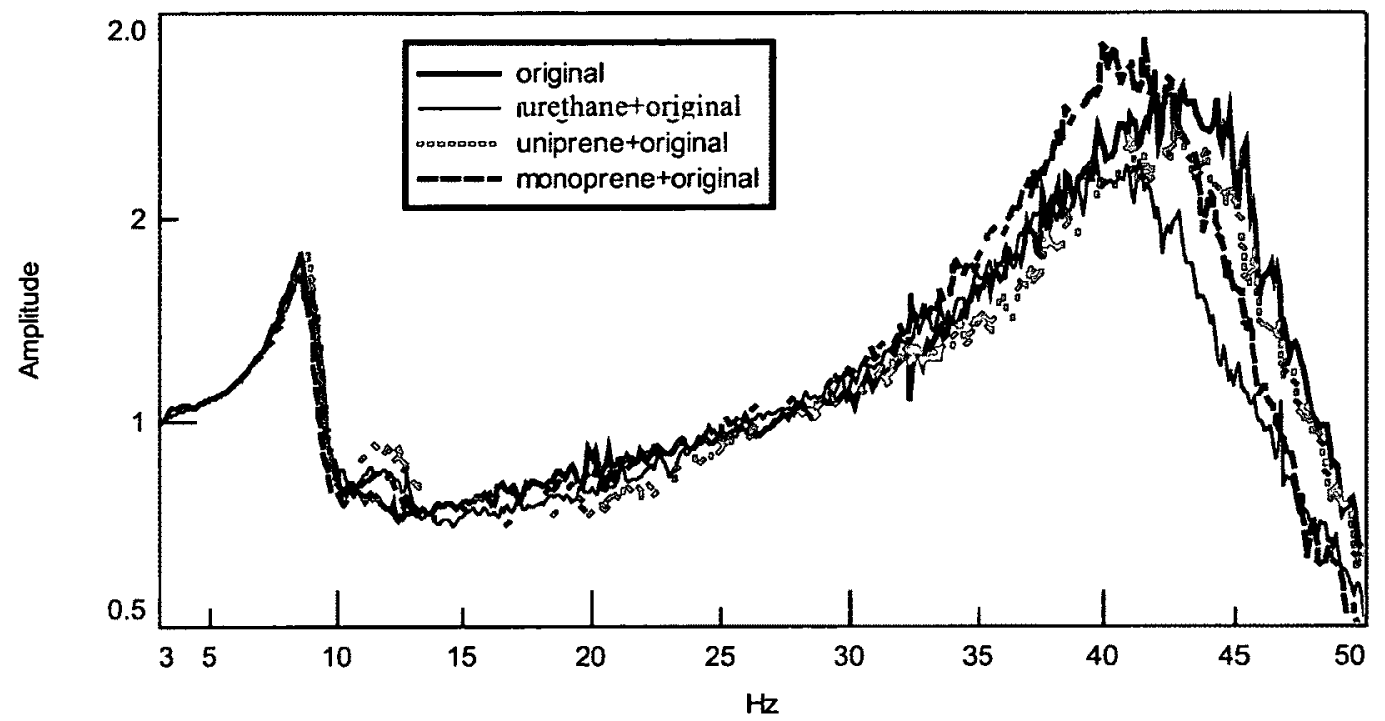

Figure 5.5: Comparison Mitigator materials using FRF in the $\mathrm{Z}$ direction

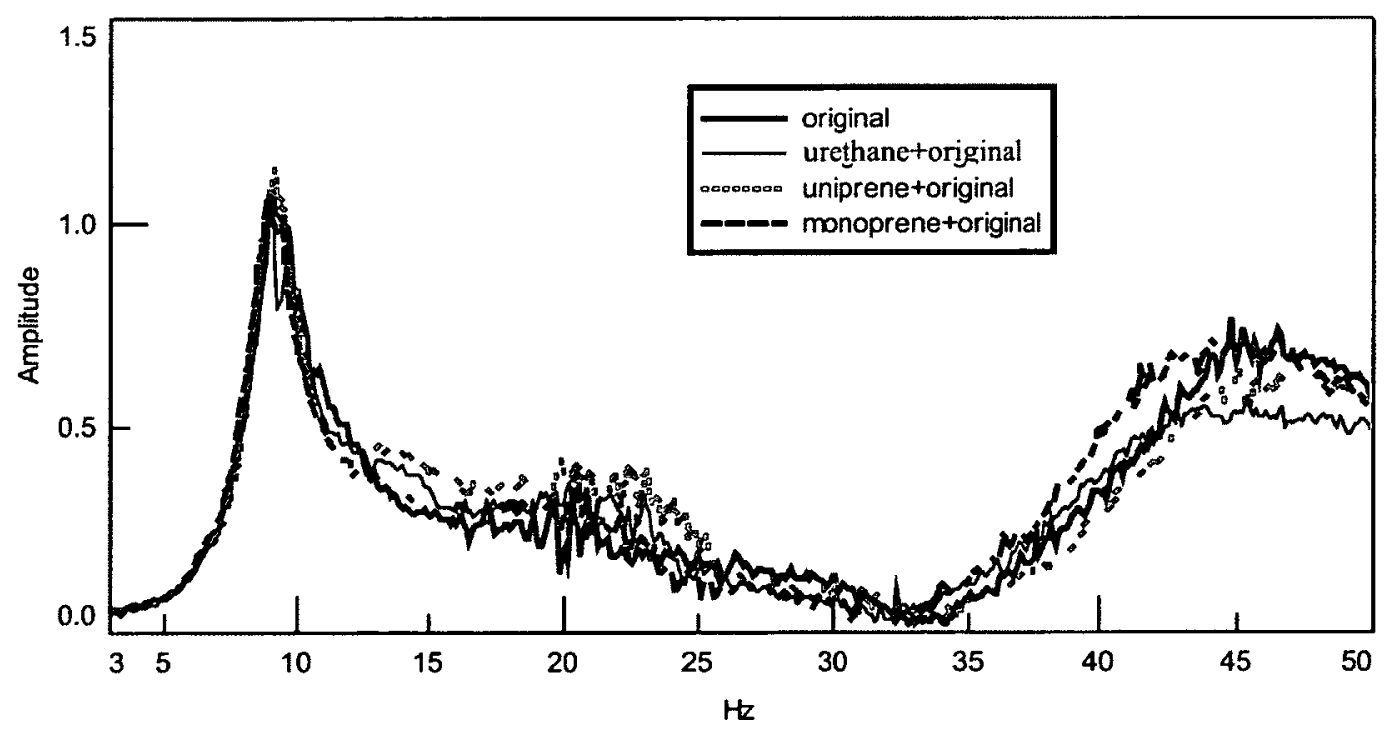

Figure 5.6: Comparison Mitigator materials using FRF in the $\mathrm{X}$ direction 
The variation in the transmissibility, particularly in the $Z$ and $X$ directions, by integrating urethane, monoprene and uniprene materials with the original cushion are shown by FRFs in Figure 5.5 and Figure 5.6, respectively. The results show that the transmissibility amplitudes vary significantly with frequency. Therefore, it is important to select the cushion material combination taking into account the forced vibration frequencies transmitted to the seat due to $\mathrm{N} / \mathrm{rev}$ rotor harmonics.

Similarly, the variation in the transmissibility in the $\mathrm{Z}$ and $\mathrm{X}$ directions by combining the original bottom cushion with the Sorbothane materials of 0.25 ", 0.5 " and 1.0" thicknesses are shown in Figure 5.7 and Figure 5.8, respectively. These results also show that the transmissibility amplitudes vary significantly with the input frequency.

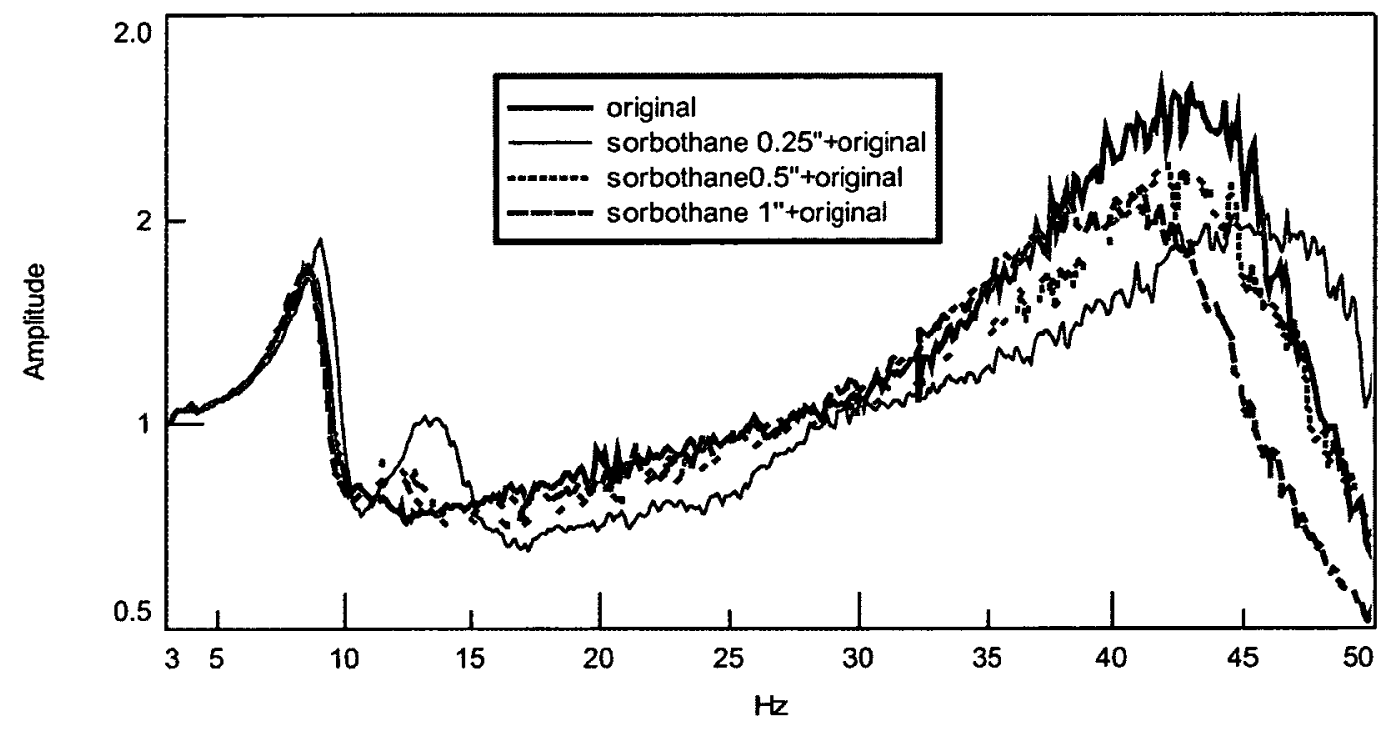

Figure 5.7: Comparison Sorbothane materials using FRF in the $\mathrm{Z}$ direction

The base excited vibration tests performed on the full-scale Bell-412 seat using the mechanical shaker were used to evaluate the vibration absorption characteristics of novel energy absorbing cushion materials, namely, Mitigator and Sorbothane. However, mechanical shaker tests were not able to confirm their vibration suppression performance on helicopter aircrew under realistic conditions in the helicopter due to several shortcomings of this preliminary investigative tool. For example, the impedance 
properties of the structural boundary condition was significantly different, specifically, the seat was clamped on to the stiff vibration table for the shaker tests while the helicopter floor was relatively flexible. In addition, the mannequins used for the shaker tests only represent the distributed weight of the aircrew and it did not sufficiently represent the characteristics of the human body. These differences, amongst others, make it impossible to represent the realistic helicopter aircrew vibration environment using mechanical shaker tests to determine the aircrew vibration performance of novel cushions confidently. Therefore, flight tests were essential to verify that the relatively small but appreciable reduction in aircrew vibration due to the integration of novel cushion materials is maintained under the realistic operating conditions of the Bell-412 helicopter.

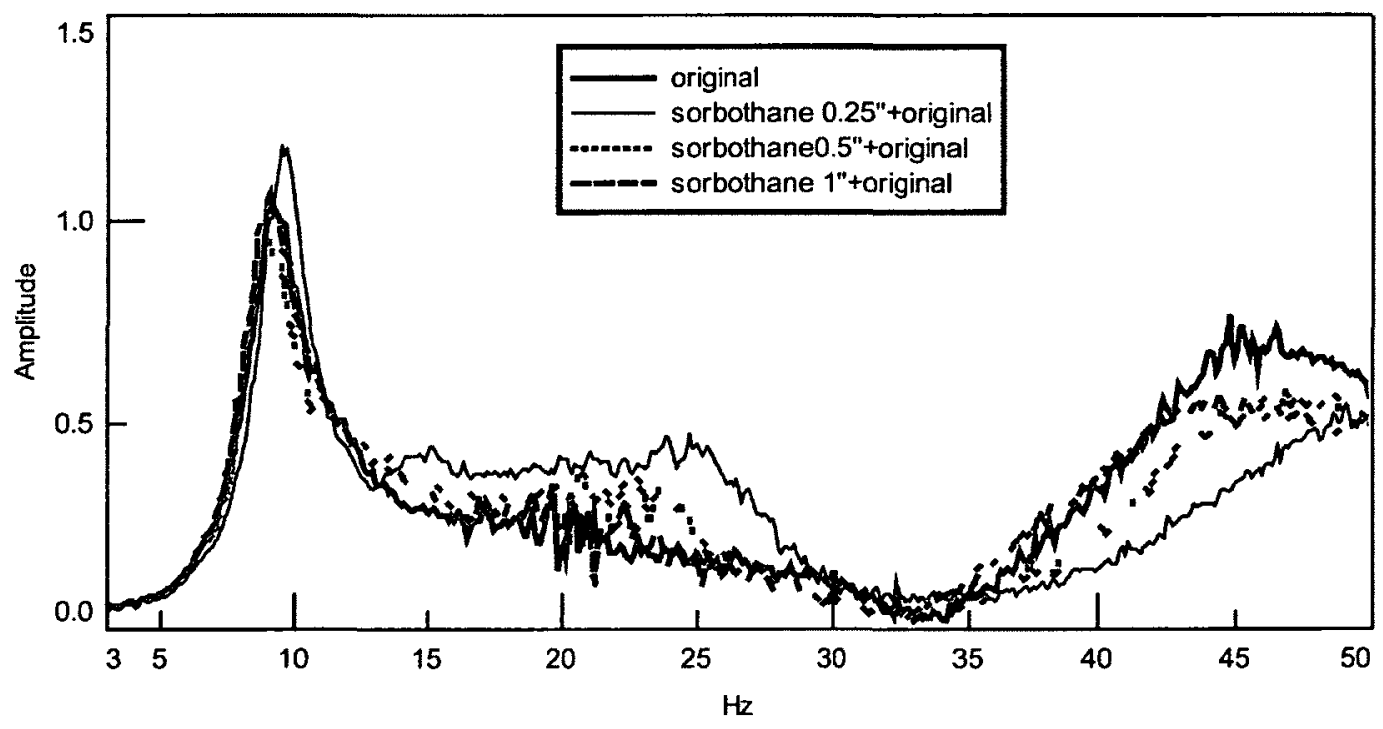

Figure 5.8: Comparison Sorbothane materials using FRF in the $\mathrm{X}$ direction

\subsection{Flight Tests to Verify Vibration Suppression Performance}

Flight tests were performed using the NRC Bell-412 helicopter to verify vibration suppression performance of novel seat cushions under typical flight conditions. The flight test plan was similar to that described in detail in Section 2.2, with instrumentation to acquire the aircrew and structural vibration data under representative flight conditions. In this investigation, the vibration levels recorded at the aircrew helmets and the seat 
cushion interfaces were evaluated to compare the vibration suppression performance of various novel cushion materials with the original cushion.

Based on material characterization tests results from the shaker tests, a total of seven seat cushion configurations were selected for the flight tests. That included the original in-service cushion as the baseline evaluation metric to compare the vibration isolation performance of the novel cushion materials. All cushion configurations were tested as part of the bottom cushion of the pilot and co-pilot seats. All energy absorbing cushion materials were tested by integrating them separately with the original Bell-412 in-service cushion by placing the novel cushion material pad on the seat pan underneath the original cushion. The selected cushion materials for the flight tests shown in Figure 5.9 included the Mitigator cushions manufactured with urethane, monoprene and uniprene materials and the Sorbothane pads with thicknesses of 1 ", 0.5 " and 0.25 ".

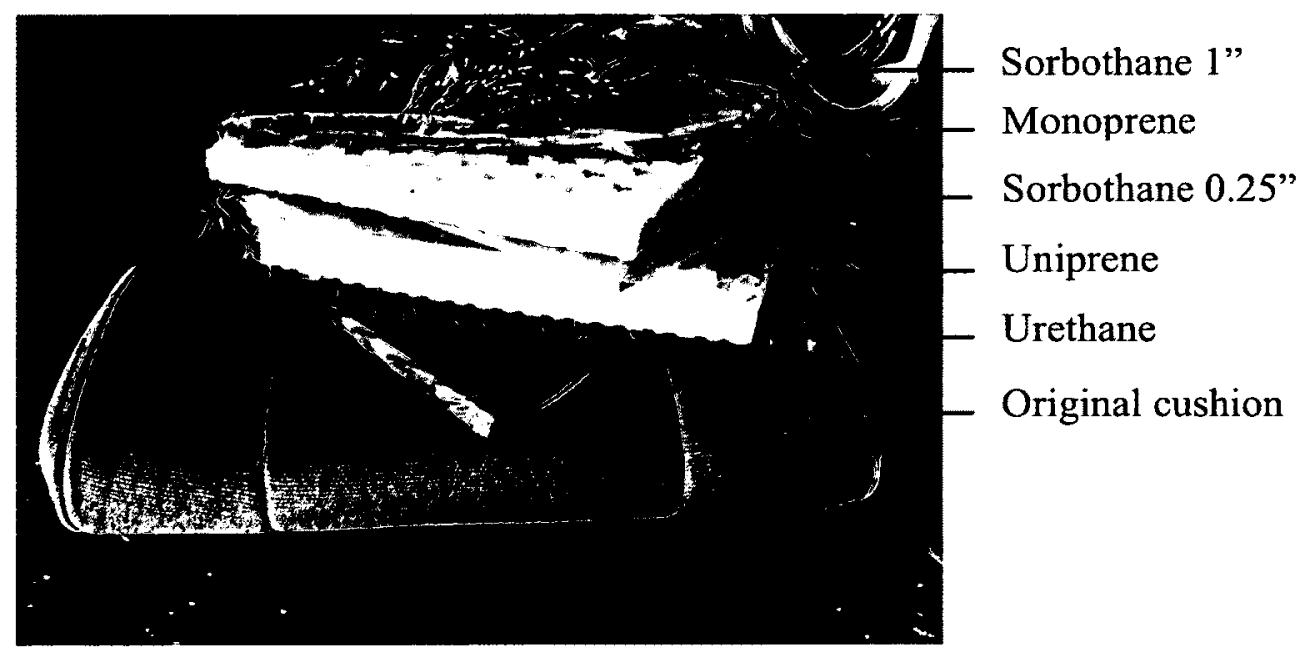

Figure 5.9: Cushions materials evaluated using flight test

The flight tests were conducted on July 8,2009 and the vibration data from all the flight conditions were acquired successfully. Each cushion configuration was tested under a full mission profile of the Bell-412 helicopter which included 8 flight conditions, namely, ground, hover, translational lift, $60 \mathrm{kts}$ climb, $120 \mathrm{kts}$ cruise, $140 \mathrm{kts}$ decent, 2-g turn and landing. The flight tests included 7 flights to evaluate all the cushion configurations on both the pilot and the co-pilot. Once a full flight profile was 
completed, the pilot would land the Bell-412 helicopter in order to change the seat cushion configuration. Since only one test sample was available for each cushion material, the test flight matrix in Table 5.5 was used to evaluate the vibration suppression performance on both the pilot and the co-pilot for all selected cushion configurations.

Table 5.5: Flight test matrix to evaluate all selected cushion configuration

\begin{tabular}{|c|c|c|}
\hline Flight No & Pilot Cushion & Co-Pilot Cushion \\
\hline Flight No 1 & Original & Urethane + Original \\
\hline Flight No 2 & 0.25 "Sorbothane + Original & Monoprene + Original \\
\hline Flight No 3 & 0.5"Sorbothane + Original & Uniprene + Original \\
\hline Flight No 4 & 1.0 "Sorbothane + Original & 0.25 "Sorbothane + Original \\
\hline Flight No 5 & Urethane + Original & 0.5"Sorbothane + Original \\
\hline Flight No 6 & Monoprene + Original & 1.0 "Sorbothane + Original \\
\hline Flight No 7 & Uniprene + Original & Original \\
\hline
\end{tabular}

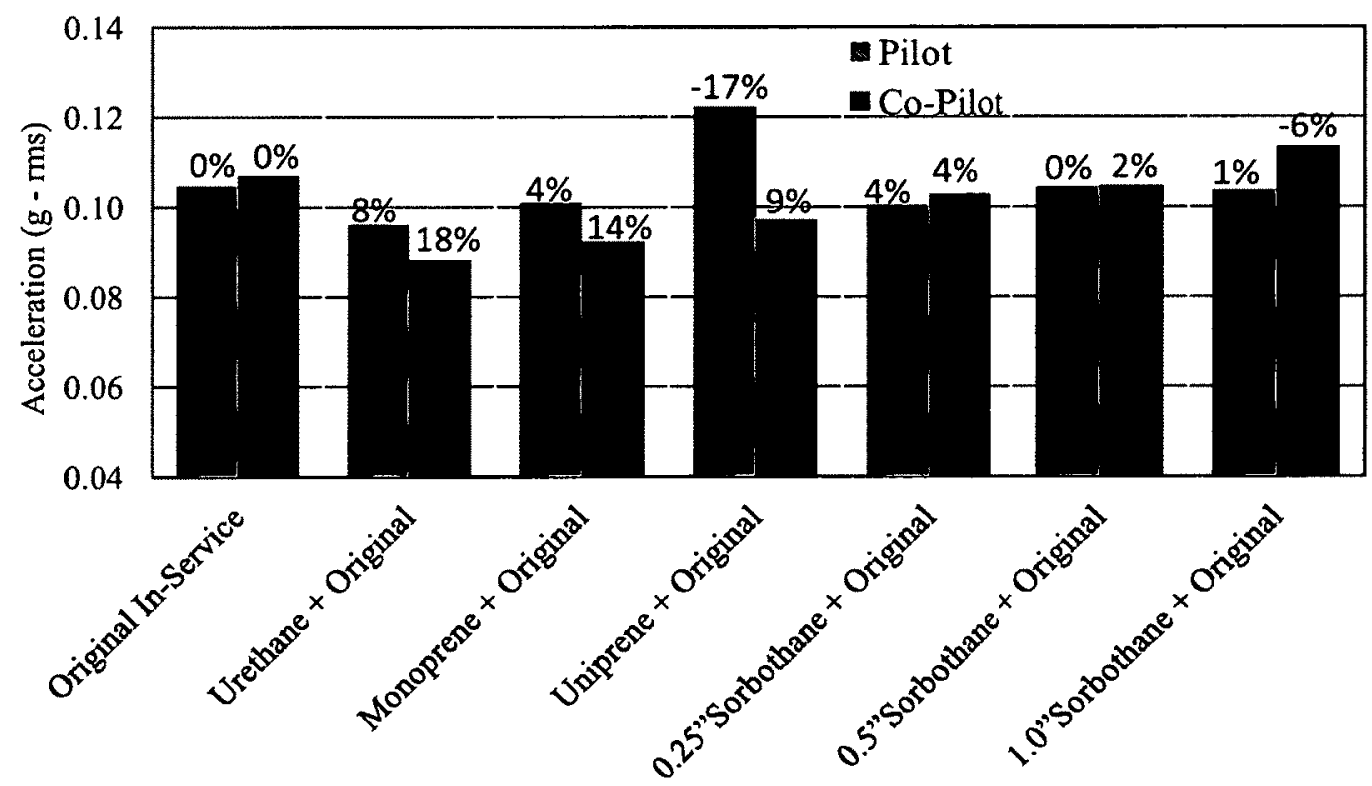

Figure 5.10: Aircrew helmet vibration level for selected cushions at $120 \mathrm{kts}$ condition

The flight tests results showed that modifying the cushion properties by inserting a novel energy absorbing material did indeed reduce the aircrew vibration. The overall grms vibration levels for the $120 \mathrm{kts}$ cruise condition with vibration reduction percentage for all tested cushion combinations, based on the aggregated acceleration measured on 
the aircrew helmets in all three directions between the frequency range of 2 and $50 \mathrm{~Hz}$, are shown in Figure 5.10. This $120 \mathrm{kts}$ cruise flight condition was used for detailed comparison analyses because typical Bell-412 helicopters operate the largest portion of their missions at this flight condition. Most cushion combinations showed a reduction in vibration measured at the pilot and co-pilot helmets while a few cushion materials showed an increase in aircrew helmet vibration. Therefore, the impedance properties of the cushion is required to be selected carefully to optimize the aircrew vibration reduction under varying flight conditions as well as account for the physical differences in aircrew.

\subsubsection{Evaluation of Sorbothane Material}

Comparison of the aircrew vibration measured during each flight condition under various Sorbothane material combinations implemented on the aircrew seat cushion is tabulated in Table 5.6. The rms acceleration measured in $\mathrm{g}$ for the pilot and co-pilot locations for each corresponding flight condition showed that the performance of the Sorbothane material varied substantially depending on flight conditions for each measurement location. Based on overall aggregated vibration measured on the aircrew helmets, combination of the original in-service seat cushion with Sorbothane material of 0.25 " thickness showed the highest vibration reduction, specifically, $4 \%$ reduction for both the pilot and the co-pilot.

Detailed frequency domain analysis was performed in order to identify the effect of Sorbothane material combinations at N/rev harmonics, particularly at $1 / \mathrm{rev}$ and $4 / \mathrm{rev}$. The spectrum measured by the pilot's cushion accelerometer in $\mathrm{Z}$ direction shown in Figure 5.11 clearly shows that all Sorbothane material combinations reduce the $4 / \mathrm{rev}$ peak while only the 0.25 " thickness pad showed a reduction for the $1 / \mathrm{rev}$ peak compared to the original cushion. The $4 / \mathrm{rev}$ peak was reduced by $31 \%, 14 \%$ and $17 \%$ for the Sorbothane material thicknesses of 0.25 ", 0.5 " and 1.0 ", respectively. In contrast, the $1 / \mathrm{rev}$ peak was reduced by $25 \%$ for the Sorbothane pad thickness of 0.25 " while the peak was amplified by $40 \%$ for both the 0.5 " and 1.0 " thick Sorbothane pads. 


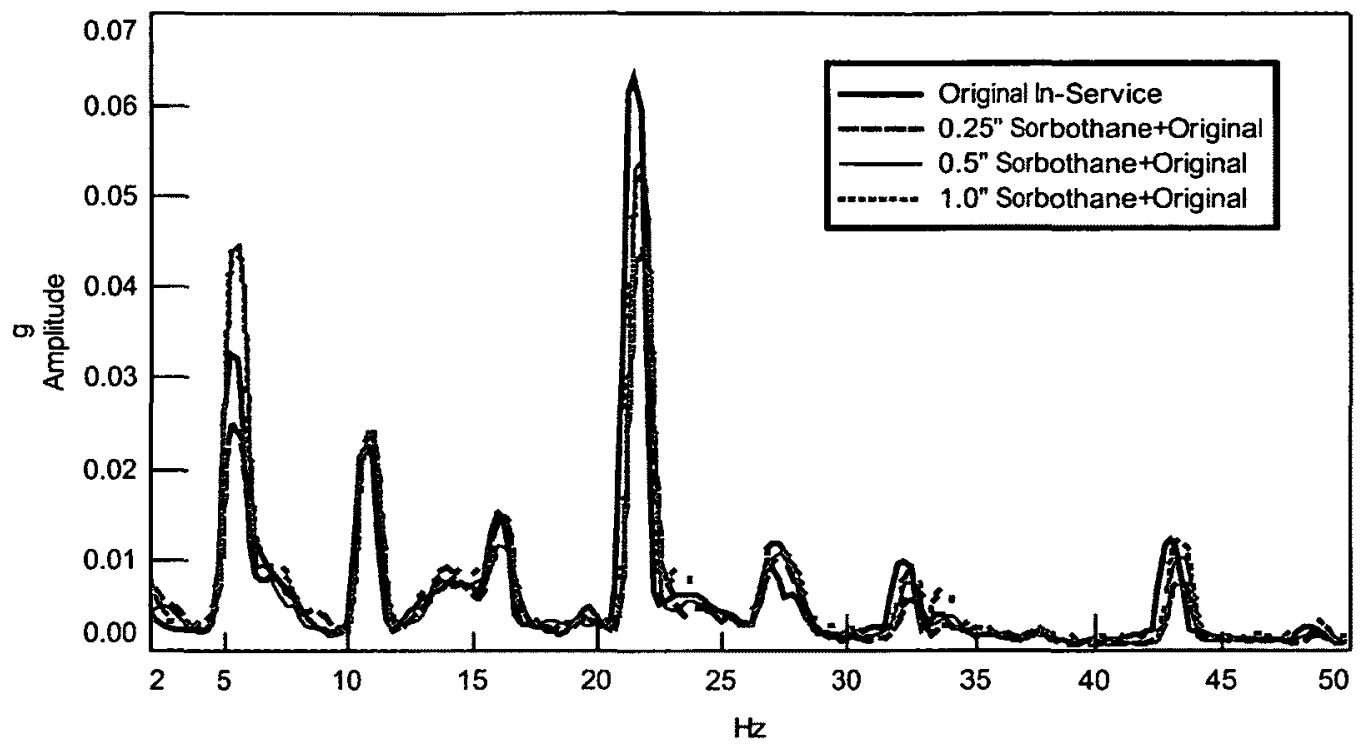

Figure 5.11: Spectra of Pilot cushion in the $\mathrm{Z}$ direction for Sorbothane material

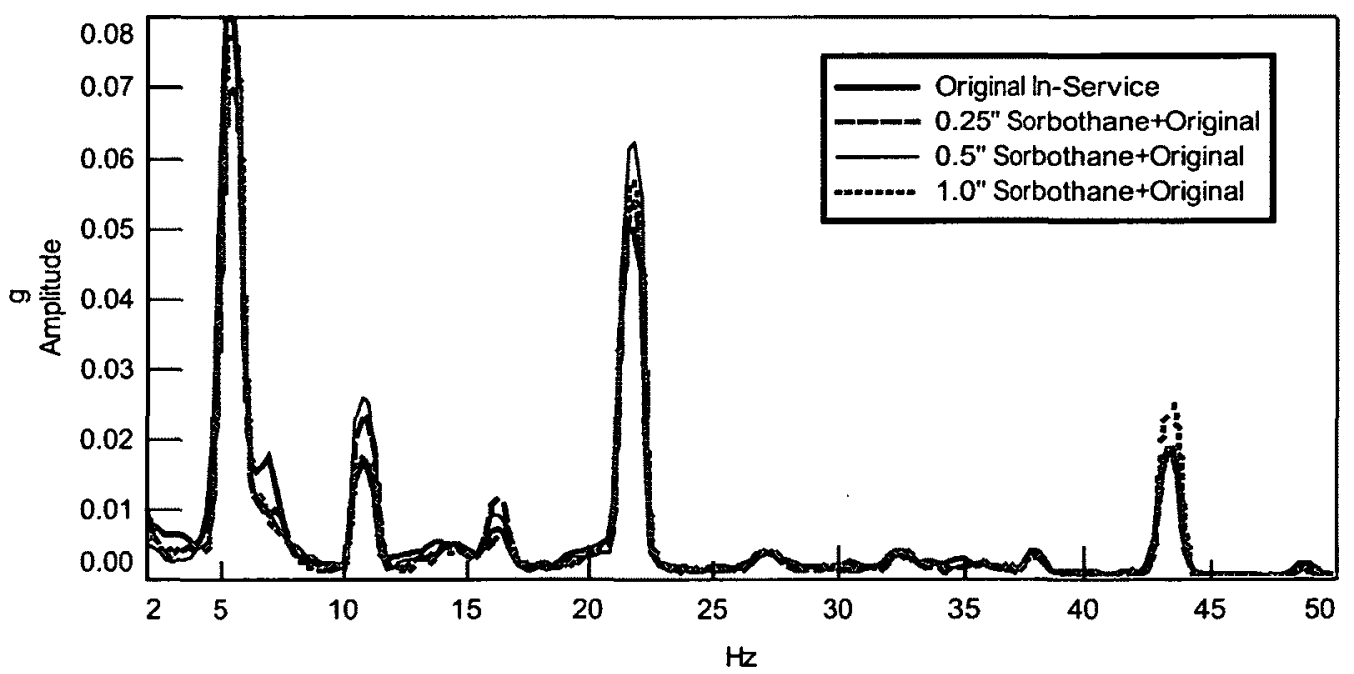

Figure 5.12: Spectra of Co-Pilot helmet in the $\mathrm{Z}$ direction for Sorbothane material

The spectrum measured by the accelerometer mounted on the co-pilot's helmet in the $\mathrm{Z}$ direction, shown in Figure 5.11, demonstrates that all Sorbothane material combinations reduce the $1 / \mathrm{rev}$ peak while they all increase the vibration level at the $4 / \mathrm{rev}$ peak. The $4 / \mathrm{rev}$ peak was increased by $8 \%, 25 \%$ and $14 \%$ while the $1 / \mathrm{rev}$ peak was reduced by $13 \%, 12 \%$ and $3 \%$ for the Sorbothane material thicknesses of 0.25 ", 0.5 " and 
1.0 ", respectively. The flight test data from the pilot's and co-pilot's helmet showed that the performance of Sorbothane cushion materials varies significantly due to differences in aircrew and flight condition. However, it is important to note that the vibration data analyzed for each flight condition was acquired by flying a single Bell-412 aircraft once at a specified flight condition with a particular pilot and co-pilot. Therefore, it is recommended that more extensive flight tests be conducted using a larger population of aircrew as well as by using several aircraft of the same type to gather statistically significant set of data to confirm the vibration suppression of Sorbothane cushion materials integrated in the Bell-412 helicopter seats.

\subsubsection{Evaluation of Mitigator Material}

Similar analyses were performed to compare the aircrew vibration measured under each flight condition for various Mitigator cushion combinations implemented on the aircrew seat cushion. The rms acceleration measured in $\mathrm{g}$ for the pilot and co-pilot locations for each corresponding flight condition, tabulated in Table 5.6, showed that the performance of the Mitigator material also varied substantially for each measurement location depending on flight conditions. Based on overall aggregated vibration measured on the aircrew helmets, combination of the original in-service Bell-412 seat cushion with a Mitigator cushion manufactured with urethane showed the highest vibration reduction, namely, $8 \%$ and $18 \%$, for the pilot and the co-pilot, respectively.

The spectrum measured by the accelerometer mounted on the pilot's helmet in the $\mathrm{Z}$ direction, shown in Figure 5.13, demonstrates that all Mitigator material combinations, namely, urethane, monoprene and uniprene reduced the $1 / \mathrm{rev}$ peak by $28 \%, 25 \%$ and $21 \%$, respectively. Although a reduction of $38 \%$ for urethane and $41 \%$ for monoprene was measured for the $4 / \mathrm{rev}$ peak, the uniprene material increased the $4 / \mathrm{rev}$ peak by $11 \%$ compared to the original cushion. Similar analysis of the co-pilot's helmet accelerometer in the $\mathrm{Z}$ direction, shown in Figure 5.14, also demonstrate that all Mitigator material combinations, namely, urethane, monoprene and uniprene reduced the $1 /$ rev peak by $30 \%, 29 \%$ and $12 \%$, respectively. Although a reduction of $7 \%$ for urethane and $8 \%$ for uniprene was measured for the $4 / \mathrm{rev}$ peak, the monoprene material marginally increased 
the $4 / \mathrm{rev}$ peak by $1 \%$ compared to the original cushion. The flight test data showed that combining the original cushion with Mitigator cushion manufactured with urethane consistently reduced the $1 /$ rev peak significantly. In addition, this urethane material was able to marginally reduce the $4 /$ rev vibration peak as well.

The vibration performance of the Mitigator cushion material with urethane was further confirmed by evaluating the spectrum measured by the accelerometer mounted on the co-pilot's helmet in the $\mathrm{X}$ direction as shown in Figure 5.15. The data shows that the urethane cushion reduced the $1 /$ rev peak by $25 \%$ and the $4 /$ rev peak by $5 \%$. Based on analysis of the aircrew vibration levels at the helmet locations, combining the original cushion with the Mitigator urethane pad consistently provided the best aircrew vibration suppression performance compared to other evaluated cushion combinations.

In order to confirm the vibration reduction performance of the Mitigator urethane material under varying flight conditions, further analyses were conducted to compare the pilot and co-pilot vibration reduction under all 8 flight conditions. Based on the aggregated rms vibration measured on the aircrew helmets in all three directions, integrating the Mitigator urethane pad with the original cushion reduced the vibration in all but one flight condition as shown in Figure 5.16. This novel energy absorbing cushion material recorded the highest helmet vibration reduction of $26 \%$ for the pilot during landing and $22 \%$ for the co-pilot during $60 \mathrm{kts}$ climb. The data showed an increase in helmet vibration only for the $2 \mathrm{~g}$ turn, which is a high vibration manoeuvre performed rarely in typical missions. In addition, the measured aircrew vibration data could be misleading because this banking manoeuvre results in a large angle on the seat which makes it difficult for the aircrew to maintain normal contact with the cushion. Due to the rare nature of this manoeuvre and measurement difficulty, the increase in aircrew vibration shown during the $2 \mathrm{~g}$ flight condition is considered inconclusive for this preliminary investigation. Therefore, it was concluded that the combination of a Bell-412 seat cushion with a Mitigator cushion manufactured with urethane reduced the aircrew helmet vibration in most flight conditions by primarily reducing the $1 / \mathrm{rev}$ peak and secondarily reducing the $4 /$ rev peak. Minor peaks, such as the $8 / \mathrm{rev}$, did not show much effect on the overall aircrew vibration levels due to the variation in cushion material. 
Table 5.6: Measured g-rms vibration levels for Sorbothane material

\begin{tabular}{|c|c|c|c|c|c|c|c|c|c|}
\hline & 竞 & 竞离 & 홀 & 章 & 容竞 & 音 & 立这 & 㫕 & 党 \\
\hline Flight Condition & \multicolumn{9}{|c|}{ Ground } \\
\hline Original in-service & 0.05 & 0.08 & 0.04 & 0.08 & \begin{tabular}{|l|}
0.06 \\
\end{tabular} & 0.03 & 0.07 & 0.04 & 0.06 \\
\hline 0.25 "Sorbothane+Original & 0.10 & 0.08 & 0.07 & 0.08 & 0.06 & 0.05 & 0.05 & 0.02 & 0.04 \\
\hline 0.5 "Sorbothane+Original & 0.10 & 0.06 & 0.07 & 0.08 & 0.04 & 0.05 & 0.07 & 0.03 & 0.07 \\
\hline 1"Sorbothane+Original & 0.14 & 0.07 & 0.10 & 0.07 & 0.05 & 0.07 & 0.07 & 0.03 & 0.05 \\
\hline Flight Condition & \multicolumn{9}{|c|}{ Hover } \\
\hline Original in-service & 0.04 & 0.05 & 0.07 & 0.12 & 0.07 & 0.05 & 0.03 & 0.02 & 0.05 \\
\hline $0.25^{\prime \prime}$ Sorbothane+Original & 0.04 & 0.03 & 0.08 & 0.11 & 0.07 & 0.04 & 0.04 & 0.03 & 0.06 \\
\hline 0.5 "Sorbothane+Original & 0.04 & 0.04 & 0.07 & 0.12 & 0.07 & 0.04 & 0.04 & 0.03 & 0.05 \\
\hline 1"SorbothanetOriginal & 0.05 & 0.02 & 0.07 & 0.13 & 0.08 & 0.05 & 0.03 & 0.03 & 0.04 \\
\hline Flight Condition & \multicolumn{9}{|c|}{ Translational Lift } \\
\hline Original in-service & 0.05 & 0.05 & 0.08 & 0.13 & 0.08 & 0.04 & 0.04 & 0.02 & 0.05 \\
\hline 0.25 "Sorbothane+ Original & 0.05 & 0.04 & 0.08 & 0.13 & 0.08 & 0.04 & 0.04 & 0.02 & 0.05 \\
\hline 0.5 "Sorbothane+Original & 0.04 & 0.04 & 0.06 & 0.11 & 0.08 & 0.04 & 0.04 & 0.03 & 0.05 \\
\hline 1"Sorbothane+Original & 0.08 & 0.03 & 0.08 & 0.11 & 0.08 & 0.04 & 0.04 & 0.02 & 0.05 \\
\hline Flight Condition & \multicolumn{9}{|c|}{$60 \mathrm{kts}$ Climb } \\
\hline Original in-service & 0.04 & 0.06 & 0.06 & 0.12 & 0.11 & 0.05 & 0.04 & 0.02 & 0.04 \\
\hline 0.25 "Sorbothane+Original & 0.04 & 0.03 & 0.05 & 0.11 & 0.10 & 0.03 & 0.03 & 0.02 & 0.04 \\
\hline 0.5 "Sorbothane+Original & 0.03 & 0.04 & 0.04 & 0.10 & 0.09 & 0.04 & 0.04 & 0.02 & 0.04 \\
\hline 1"Sorbothane+Original & 0.04 & 0.02 & 0.03 & 0.09 & 0.10 & 0.03 & 0.05 & 0.02 & 0.05 \\
\hline Flight Condition & \multicolumn{9}{|c|}{120 kts Level } \\
\hline Original in-service & 0.07 & 0.06 & 0.05 & 0.13 & 0.13 & 0.06 & 0.07 & 0.04 & 0.08 \\
\hline 0.25 "Sorbothane + Original & 0.07 & 0.05 & 0.05 & 0.13 & 0.14 & 0.05 & 0.07 & 0.03 & 0.07 \\
\hline $0.5^{\prime \prime}$ Sorbothane+Original & 0.07 & 0.05 & 0.06 & 0.13 & 0.13 & 0.06 & 0.06 & 0.04 & 0.07 \\
\hline 1"Sorbothane+Original & 0.08 & 0.04 & 0.06 & 0.12 & 0.13 & 0.06 & 0.08 & 0.03 & 0.08 \\
\hline Flight Condition & \multicolumn{9}{|c|}{ 2g Turn } \\
\hline Original in-service & 0.07 & 0.07 & 0.08 & 0.18 & 0.16 & 0.08 & 0.06 & 0.04 & 0.08 \\
\hline 0.25 "Sorbothane+Original & 0.10 & 0.07 & 0.10 & 0.17 & 0.19 & 0.09 & 0.08 & 0.05 & 0.11 \\
\hline 0.5 "Sorbothane+Original & 0.10 & 0.07 & 0.08 & 0.16 & 0.18 & 0.10 & 0.07 & 0.05 & 0.10 \\
\hline 1"Sorbothane+Original & 0.10 & 0.05 & 0.09 & 0.15 & 0.17 & 0.10 & 0.08 & 0.04 & 0.09 \\
\hline Flight Condition & \multicolumn{9}{|c|}{140 kts Descend } \\
\hline Original in-service & 0.08 & 0.08 & 0.09 & 0.16 & 0.17 & 0.08 & 0.07 & 0.04 & 0.09 \\
\hline $0.25^{\prime \prime}$ Sorbothane+Original & 0.10 & 0.07 & 0.11 & 0.17 & 0.18 & 0.08 & 0.07 & 0.05 & 0.09 \\
\hline 0.5 "Sorbothane+Original & 0.08 & 0.04 & 0.05 & 0.12 & 0.15 & 0.06 & 0.07 & 0.04 & 0.08 \\
\hline 1"Sorbothane+Original & 0.08 & 0.03 & 0.06 & 0.11 & 0.14 & 0.06 & 0.08 & 0.04 & 0.08 \\
\hline Flight Condition & \multicolumn{9}{|c|}{ Landing } \\
\hline Original in-service & 0.06 & 0.08 & 0.15 & 0.18 & 0.11 & 0.09 & 0.06 & 0.03 & 0.10 \\
\hline $0.25^{\prime \prime}$ Sorbothane+Original & 0.06 & 0.05 & 0.15 & 0.14 & 0.10 & 0.08 & 0.05 & 0.03 & 0.09 \\
\hline 0.5 "Sorbothane+Original & 0.09 & 0.04 & 0.10 & 0.13 & 0.09 & 0.07 & 0.06 & 0.04 & 0.09 \\
\hline 1"Sorbothane+Original & 0.07 & 0.03 & 0.12 & 0.13 & 0.10 & 0.09 & 0.06 & 0.03 & 0.08 \\
\hline
\end{tabular}


Table 5.7: Measured g-rms vibration levels for Mitigator material

\begin{tabular}{|c|c|c|c|c|c|c|c|c|c|}
\hline & 咅蒂 & 竞 & 咅离 & 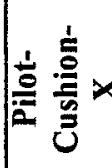 & $\mid \frac{\dot{1}}{\frac{1}{2}}$ & N & 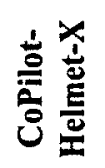 & 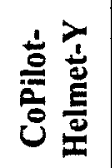 & 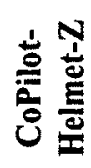 \\
\hline Flight Condition & \multicolumn{9}{|c|}{ Ground } \\
\hline Original in-service & 0.05 & 0.08 & 0.04 & 0.08 & 0.06 & 0.03 & 0.07 & 0.04 & 0.06 \\
\hline Urethane+Original & 0.06 & 0.03 & 0.04 & 0.06 & 0.04 & 0.03 & 0.07 & 0.02 & 0.07 \\
\hline Monoprene+Original & 0.10 & 0.02 & 0.07 & 0.06 & 0.06 & 0.04 & 0.08 & 0.04 & 0.07 \\
\hline Uniprene+Original & 0.08 & 0.05 & 0.05 & 0.07 & 0.05 & 0.03 & 0.10 & 0.04 & 0.10 \\
\hline Flight Condition & \multicolumn{9}{|c|}{ Hover } \\
\hline Original in-service & 0.04 & 0.05 & 0.07 & 0.12 & 0.07 & 0.05 & 0.03 & 0.02 & 0.05 \\
\hline Urethane+Original & 0.05 & 0.02 & 0.07 & 0.11 & 0.07 & 0.04 & 0.04 & 0.02 & 0.05 \\
\hline Monoprene+Original & 0.05 & 0.02 & 0.06 & 0.10 & 0.08 & 0.04 & 0.03 & 0.02 & 0.05 \\
\hline Uniprene+Original & 0.04 & 0.03 & 0.06 & 0.11 & $\begin{array}{l}0.08 \\
\end{array}$ & 0.04 & 0.03 & 0.03 & 0.05 \\
\hline Flight Condition & \multicolumn{9}{|c|}{ Translational Lift } \\
\hline Original in-service & 0.05 & 0.05 & 0.08 & 0.13 & 0.08 & 0.04 & 0.04 & 0.02 & 0.05 \\
\hline Urethane+Original & 0.05 & 0.03 & 0.06 & 0.11 & 0.08 & 0.04 & 0.04 & 0.02 & 0.05 \\
\hline Monoprene+Original & 0.06 & 0.02 & 0.07 & 0.11 & 0.08 & 0.04 & 0.03 & 0.02 & 0.04 \\
\hline Uniprene+Original & 0.06 & 0.03 & 0.06 & 0.10 & 0.08 & 0.03 & 0.04 & 0.03 & 0.05 \\
\hline Flight Condition & \multicolumn{9}{|c|}{60 kts Climb } \\
\hline Original in-service & 0.04 & 0.06 & 0.06 & 0.12 & 0.11 & 0.05 & 0.04 & 0.02 & 0.04 \\
\hline Urethane+Original & 0.07 & 0.03 & 0.04 & 0.08 & 0.09 & 0.03 & 0.03 & 0.02 & 0.03 \\
\hline Monoprene+Original & 0.06 & 0.03 & 0.05 & 0.08 & 0.11 & 0.04 & 0.03 & 0.02 & 0.04 \\
\hline Uniprene+Original & 0.06 & 0.02 & 0.05 & 0.09 & 0.09 & 0.03 & 0.03 & 0.02 & 0.04 \\
\hline Flight Condition & \multicolumn{9}{|c|}{120 kts Level } \\
\hline Original in-service & 0.07 & 0.06 & 0.05 & 0.13 & 0.13 & 0.06 & 0.07 & 0.04 & 0.08 \\
\hline Urethane+Original & 0.07 & 0.03 & 0.06 & 0.10 & 0.13 & 0.06 & 0.05 & 0.04 & 0.06 \\
\hline Monoprene+Original & 0.08 & 0.03 & 0.05 & 0.10 & 0.14 & 0.06 & 0.06 & 0.03 & 0.06 \\
\hline Uniprene+Original & 0.09 & 0.04 & 0.07 & 0.12 & 0.14 & 0.05 & 0.06 & 0.03 & 0.07 \\
\hline Flight Condition & \multicolumn{9}{|c|}{$2 \mathrm{~g}$ Turn } \\
\hline Original in-service & 0.07 & 0.07 & 0.08 & 0.18 & 0.16 & 0.08 & 0.06 & 0.04 & 0.08 \\
\hline Urethane+Original & 0.10 & 0.06 & 0.09 & 0.16 & 0.19 & 0.10 & 0.07 & 0.04 & 0.10 \\
\hline Monoprene+Original & 0.09 & 0.05 & 0.08 & 0.16 & 0.20 & 0.10 & 0.07 & 0.04 & 0.09 \\
\hline Uniprene+Original & 0.09 & 0.05 & 0.07 & 0.18 & 0.16 & 0.07 & 0.07 & 0.04 & 0.10 \\
\hline Flight Condition & \multicolumn{9}{|c|}{140 kts Descend } \\
\hline Original in-service & 0.08 & 0.08 & 0.09 & 0.16 & 0.17 & 0.08 & 0.07 & 0.04 & 0.09 \\
\hline Urethane+Original & 0.10 & 0.04 & 0.08 & 0.13 & 0.17 & 0.08 & 0.06 & 0.04 & 0.08 \\
\hline Monoprene+Original & 0.12 & 0.04 & 0.07 & 0.13 & 0.16 & 0.07 & 0.05 & 0.03 & 0.07 \\
\hline Uniprene+Original & 0.09 & 0.04 & 0.07 & 0.13 & 0.15 & 0.06 & 0.06 & 0.03 & 0.07 \\
\hline Flight Condition & \multicolumn{9}{|c|}{ Landing } \\
\hline Original in-service & 0.08 & 0.15 & 0.18 & 0.11 & 0.09 & 0.06 & 0.03 & 0.10 & 0.08 \\
\hline Urethane+Original & 0.04 & 0.11 & 0.14 & 0.11 & 0.08 & 0.06 & 0.04 & 0.08 & 0.04 \\
\hline Monoprene+Original & 0.04 & 0.12 & 0.12 & 0.11 & 0.07 & 0.04 & 0.02 & 0.07 & 0.04 \\
\hline Uniprene+Original & 0.05 & 0.12 & 0.14 & 0.10 & 0.07 & 0.05 & 0.03 & 0.09 & 0.05 \\
\hline
\end{tabular}




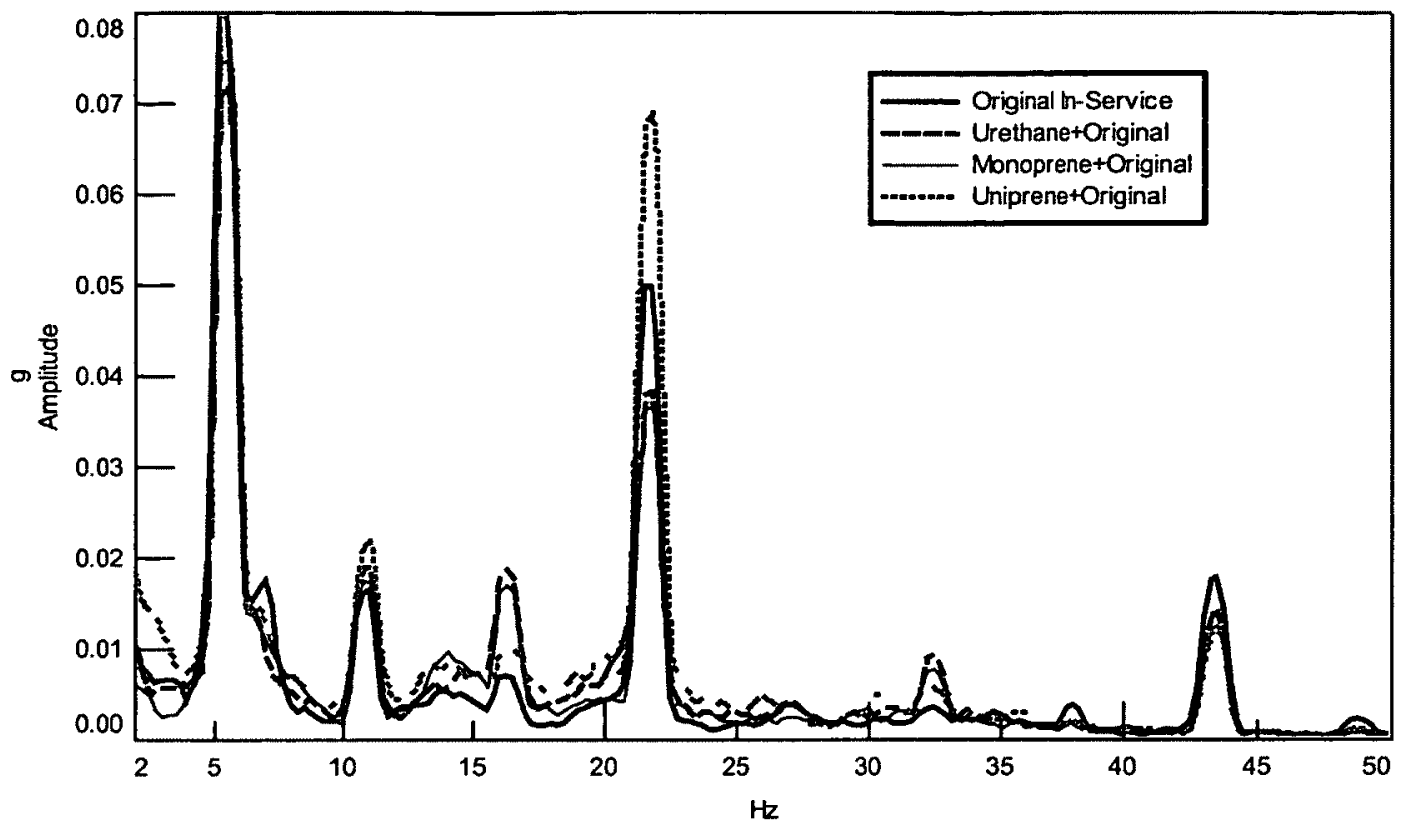

Figure 5.13: Spectra of Pilot cushion in the $\mathrm{Z}$ direction for Mitigator material

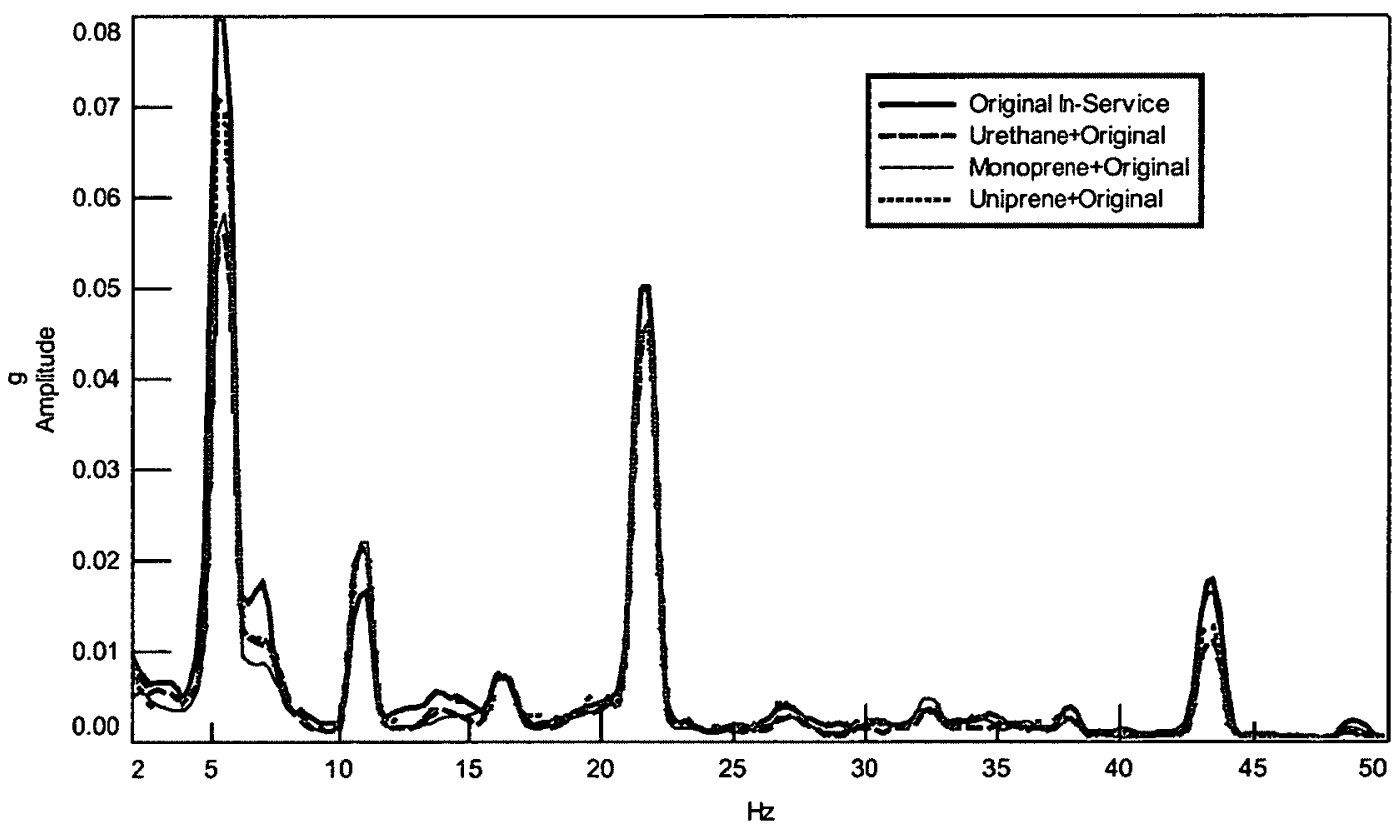

Figure 5.14: Spectra of Co-Pilot cushion in the $\mathrm{Z}$ direction for Mitigator material 


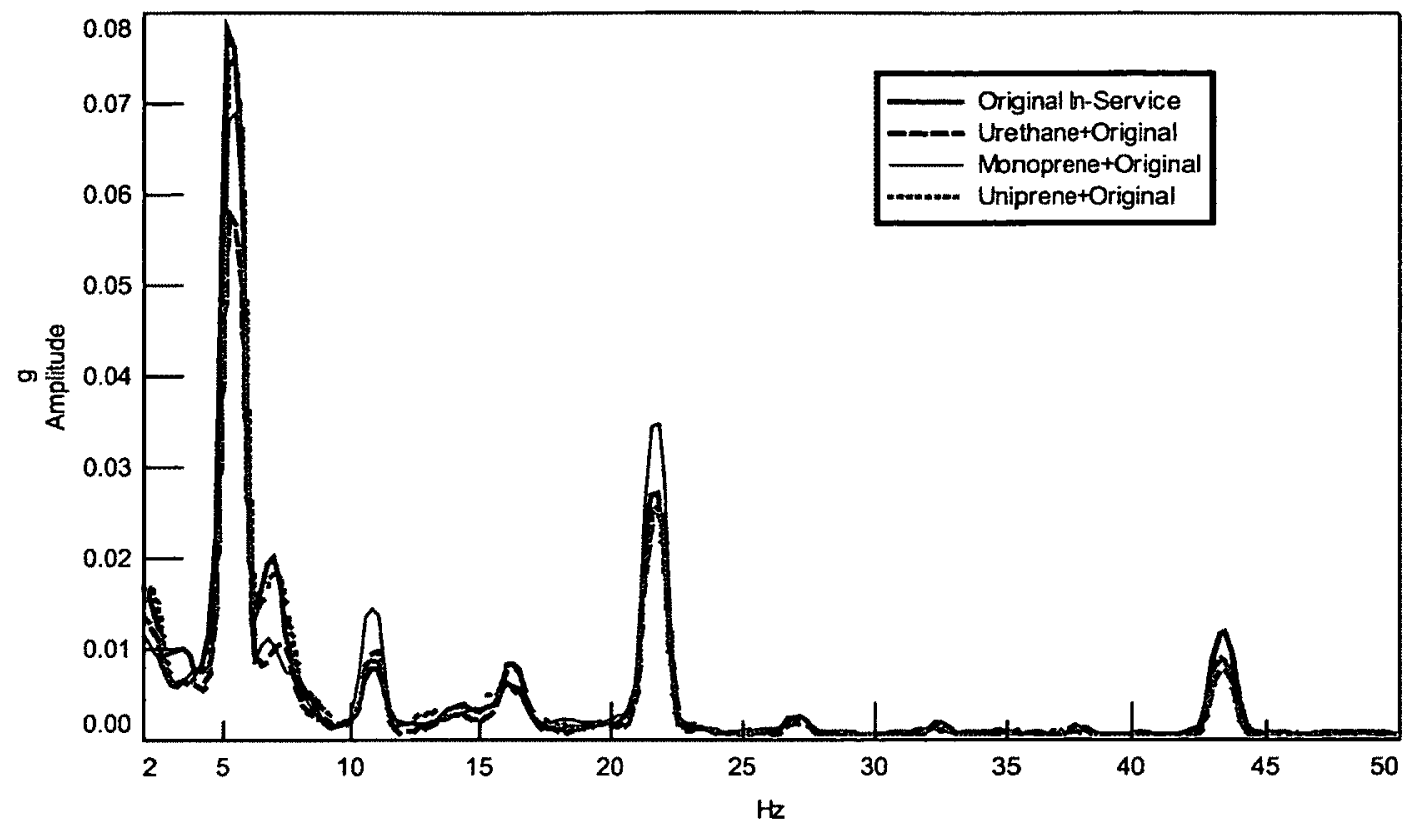

Figure 5.15: Spectra of Co-Pilot cushion in the X-direction for Mitigator material

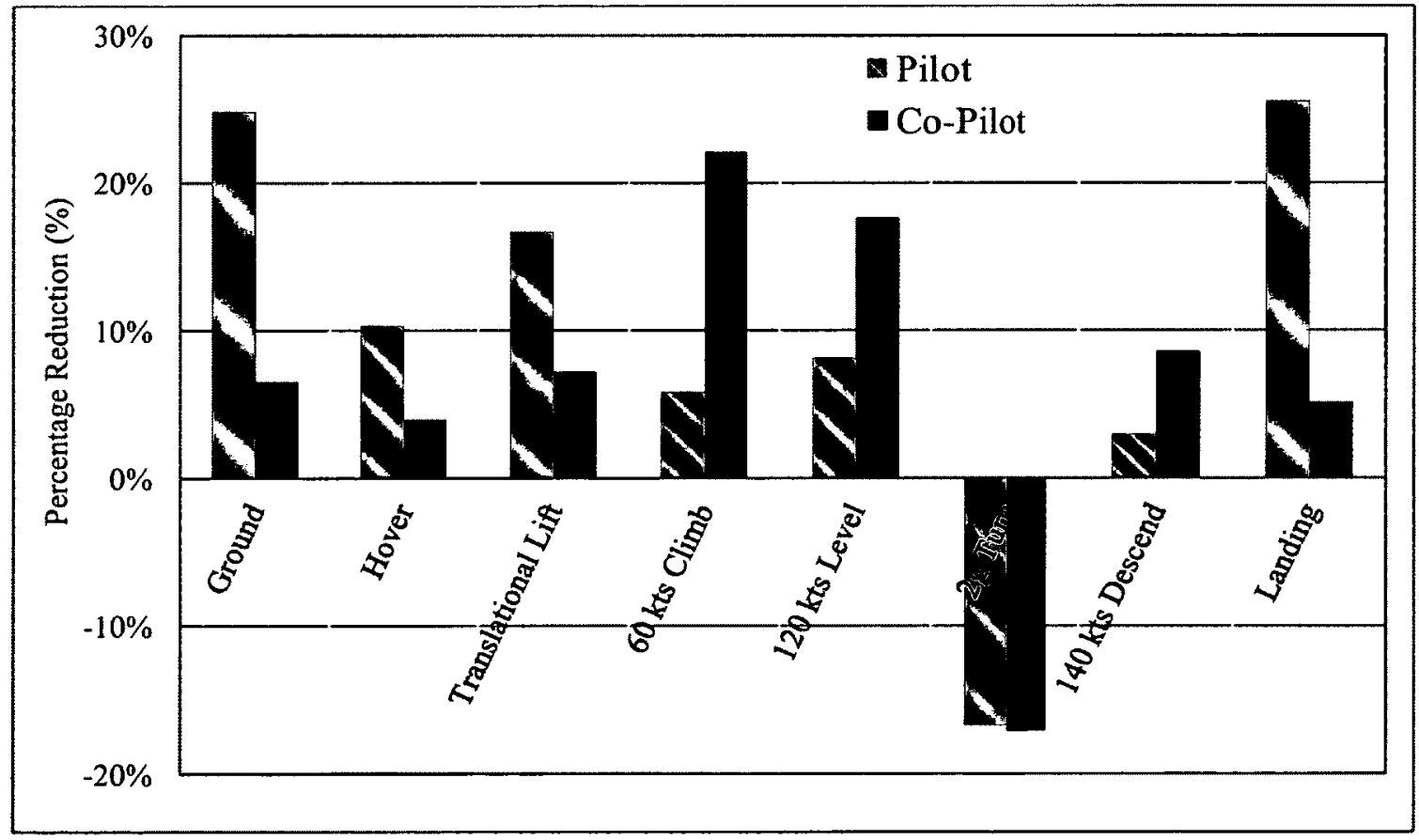

Figure 5.16: Aircrew helmet vibration with Mitigator urethane for all flight conditions 
The flight test results demonstrated that the combination of an original Bell-412 seat cushion together with a commercially available Mitigator urethane pad reduced aircrew vibration under varying flight conditions. The energy absorbing cushion materials were able to reduce the amplification measured in low frequencies, particularly in the $1 /$ rev peak as intended in the objective of this study. Therefore, it is recommended to investigate the integration of Mitigator urethane pads with the original seat cushions in the Bell-412 helicopters as a low cost solution with less certification effort.

\subsection{Summary and Conclusions}

The flight tests demonstrated that the existing in-service cushion used in the Bell-412 helicopter seat amplified the $1 /$ rev peak, while it significantly reduced the high frequency vibration. Therefore, a novel energy absorbing cushion material, namely, the Hybrid Air Cushioning System known as Mitigator and a viscoelastic polyurethane material known as Sorbothane, were investigated as a low cost solution to reduce the aircrew vibration in the Bell-412 helicopters. The mechanical shaker tests were performed to characterize the cushion material properties and the flight tests on the NRC Bell-412 helicopter were used to validate the selected novel cushion materials.

Despite the preliminary nature of this investigation, which requires more thorough study and verifications, the results confirmed that the aircrew helmet vibration levels can be effectively reduced through proper optimization of the seat cushion impedance properties. Although flight tests demonstrated inconsistent vibration suppression performance for the Sorbothane material, combining the original cushion with the Mitigator manufactured with urethane material consistently reduced the aircrew vibration under varying flight conditions and aircrew. This particular cushion combination significantly reduced the $1 / \mathrm{rev}$ peak while marginally reducing the $4 / \mathrm{rev}$ peak as well. However, it is recommended that more extensive flight tests be conducted by a larger population of aircrew as well as by using several aircraft of the same type to gather statistically significant set of data to confirm the vibration suppression of the Mitigator urethane material. 
One of the main contributions from this research is the selection of the Hybrid Air Cushioning System known as Mitigator manufactured with urethane material as an effective energy absorbing seat cushion layer to reduce the helicopter aircrew exposure to vibration. Therefore, it is recommended to pursue the integration of the Mitigator urethane pads with the original seat cushions in the Bell-412 helicopters as a suitable low cost solution with minimum certification effort to reduce the vibration transmitted to the aircrew to mitigate associated adverse health impacts due to long-term vibration exposure. 


\section{Chapter 6}

\section{Conclusions and Recommendations}

Helicopters are known to expose the aircrew to significantly high levels of whole body vibration that leads to undesirable occupational health hazards. Exposure to this high vibration environment not only leads to short-term impacts such as discomfort and fatigue but also known to cause injuries such as neck strain and back pain due to longterm exposure as documented by the rotary aircrew communities.

This research study investigated the use of novel active as well as passive methodologies to mitigate the vibration transmitted to the aircrew. In particular, this research effort developed a unique adaptive seat mount approach based on active vibration control technology, using stacked piezoelectric actuators with an adaptive feedforward algorithm, to counteract forced vibration harmonics of the helicopter main rotor. Due to significantly less certification effort required to modify the seat structure, application of active control technology to the seat structure is more practical compared to implementing such active control systems on flight critical components such as the main rotor to suppress vibration. In addition, this investigation also evaluated the use of novel energy absorbing cushion materials as a low cost, near term, solution to dampen the resonance vibration in the helicopter aircrew seat application. These hybrid vibration suppression approaches based on active and passive methodologies were implemented on a full-scale Bell-412 helicopter seat to evaluate their effectiveness under representative vibratory conditions. 


\subsection{Conclusions}

This research represents the first known investigation into the use of stacked piezoelectric actuator based adaptive mount methodology on full-scale helicopter seats to suppress aircrew vibration. Extensive biodynamic modeling effort was used to develop the design and comprehensive experimental investigations were used for performance verifications. In addition, a unique flight test was performed on the Bell-412 helicopter to verify the high levels of vibration exposure in helicopter aircrew in flight. Furthermore, vibration suppression performance of a novel energy absorbing cushion material was also demonstrated through Bell-412 helicopter flight tests. As such, this research study has generated three major conclusions as discussed in following subsections.

\subsubsection{Confirmed the Viability of the Adaptive Seat Mount Methodology for the Helicopter Aircrew Vibration Suppression}

This research effort began with an investigation into the feasibility of using active control technology to counteract vibration transmitted to the helicopter aircrew. A unique adaptive seat mount approach was successfully implemented on a full-scale Bell-412 helicopter seat by incorporating two stacked piezoelectric actuators as active struts. This design increased the structural stiffness of the seat to avoid the resonance at low frequency while generating sufficient forces to counteract harmonic forced vibration at high frequencies. A real-time controller implemented with a feed-forward algorithm based on adaptive notches counteracted the forced vibration peaks while a robust feedback control algorithm suppressed the resonance modes. Closed-loop control experiments performed using shaker tests demonstrated that the adaptive seat mount approach provided significant reduction in harmonic excitation as well as reducing the resonant modes under representative helicopter vibration spectra. The small physical size of the piezoelectric stacked actuators and the use of low weight power electronics make this novel adaptive mount approach suitable for the helicopter integration. This investigation concluded that the proposed adaptive seat mount approach based on active 
control technology is a viable solution for the helicopter seat vibration control application.

\subsubsection{Implemented a real-time controller using a feed-forward algorithm} and a feedback control law simultaneously

Another important conclusion of this research was the successful implementation of realtime adaptive controller on a full-scale helicopter seat system and the performance of the control approach was verified using mechanical shaker tests that represented helicopter floor vibration. The real-time controller simultaneously implemented a feed-forward based on Filtered-x Least Means Square algorithm to reduce harmonic excitation and a feedback control algorithm based on Linear Quadratic Gaussian regulator to suppress the resonance modes of the seat. Since the harmonic frequencies in aircrew excitation were estimated on-line and selectively included in the reference signal, the algorithm does not only suppress vibration modes selectively, but also tracks the change in the vibration spectra. The feedback controller suppressed the resonant modes of the seated mannequin that were excited due to random vibration. Closed-loop control experiments performed using mechanical shaker tests on a full-scale helicopter seat verified that the proposed real-time control algorithm that included a feed-forward and a feedback control algorithm simultaneously reduced $\mathrm{N} / \mathrm{rev}$ harmonic peaks of the rotor as well as suppressed the resonant modes of the seat system.

\subsubsection{Verified the High Levels of Vibration Exposure in Helicopter} Aircrew in Flight

Although vibration data on helicopter structures, such as the floor, have been published

previously, vibration data on aircrew, such as the helmet, was not found in published literature. Therefore, a unique flight test was performed on a Bell-412 helicopter to acquire vibration data from aircrew under typical flight profile. The flight test data demonstrated that the helicopter aircrew is exposed to significantly high levels of vibration during a variety of flight conditions and that the spectrum profile varied 
substantially depending on operating conditions as well as the aircrew configurations. The comfort assessment and health guidance based on ISO 2631 analysis indicated that most Bell-412 flight conditions were considered "uncomfortable" and "caution" while the $140 \mathrm{kts}$ flight condition was rated as "very uncomfortable" and "not safe," respectively. In contrast to the structural vibrations which showed $4 / \mathrm{rev}$ as the dominant peak, the vibration measured on the aircrew showed that the $1 / \mathrm{rev}$ as the dominant peak indicated that the seated aircrew amplified the low frequency vibration due to resonance while attenuating the high frequencies. This is a major concern for aircrew health because high levels of low frequency vibration reportedly lead to potential injuries such as neck strain and back pain under long-term exposure. The flight test data verified that the helicopter aircrews are exposed to high levels of vibration over broad operating flight conditions. Furthermore, the aircrew data measured in flight test clearly concluded the necessity to investigate seat vibration reduction solutions to mitigate the harmful effects of vibration on aircrew health.

\subsubsection{Demonstrated the Suitability of Novel Energy Absorbing Cushion Material for Helicopter Seat Application}

The flight tests demonstrated that the existing original cushion used in the Bell-412 helicopter seat system amplified the $1 / \mathrm{rev}$ peak at $5.4 \mathrm{~Hz}$, although it significantly damped the high frequency vibration such as the $4 / \mathrm{rev}$ at $21.5 \mathrm{~Hz}$ and $8 / \mathrm{rev}$ at $43.2 \mathrm{~Hz}$. Therefore, the properties of the original cushion material was used for high frequency vibration reduction while a novel energy absorbing cushion material was investigated to dampen the resonance vibration. Optimization of the seat cushion material properties was determined as a low cost solution with less certification effort to mitigate aircrew vibration. The material characterization tests conducted on the mechanical shaker and performance verification tests using Bell-412 flight trials showed that the impedance properties of the cushion required to be optimized properly in order to suppress resonance vibration at $1 / \mathrm{rev}$ peak under varying vibratory environment. The flight tests performed on the NRC Bell-412 to evaluate cushion performance demonstrated that combining the 
original cushion with the Mitigator pad manufactured with urethane material consistently reduced the aircrew vibration. The Mitigator is an engineered cushion that incorporates unique a Hybrid Air Cushioning System designed to transfer the vertical impact energy through a series of internal bleed ports and horizontal passages. This selected cushion combination significantly dampened the amplification of $1 / \mathrm{rev}$ peak while marginally reducing the 4/rev peak as well, under varying flight conditions on diverse aircrew. Although integration of novel optimization of cushion properties provided relatively lower level of vibration suppression compared to modifications to the seat structure, it was selected as the most practical and low cost solution with minimum certification effort to reduce seat vibration. Therefore, it was concluded that integrating novel energy absorbing materials in helicopter seats is a suitable near-term solution to mitigate aircrew exposure to vibration.

\subsection{Recommendations}

Although this research has successfully shown the technical feasibility and potential benefits of an adaptive seat mount approach and novel energy absorbing cushion materials for helicopter seat vibration suppression applications, a number of challenges must be overcome through further refinements prior to their practical implementation. The following subsections identify several areas of future research work and potential enhancements in order to advance the technology readiness level of these novel methodologies to improve the health of helicopter aircrew by reducing the level of vibration exposure.

\subsubsection{Refinement of the Adaptive Seat Mount Design}

The adaptive seat mount approach has shown the viability to reduce aircrew vibration levels without significant modification to the helicopter structure or critical flight components. However, technical challenges regarding this novel adaptive seat mount design to maintain crashworthiness of the seat must be addressed before this active control concept can be implemented into the helicopter fleet. Adaptive mount design 
used piezoelectric stacked actuators as active struts on both sides of the seat to connect the pan to the base of the frame. This design assumes that shear pins could be integrated into the attachment mechanisms to allow the seat pan to move downwards freely in case of a crash to meet the crashworthiness criteria of the helicopter seat design. This assumption in the mechanical design must be validated to ensure that the crashworthiness requirement of the seat is not compromised due to the integration of the adaptive mount concept.

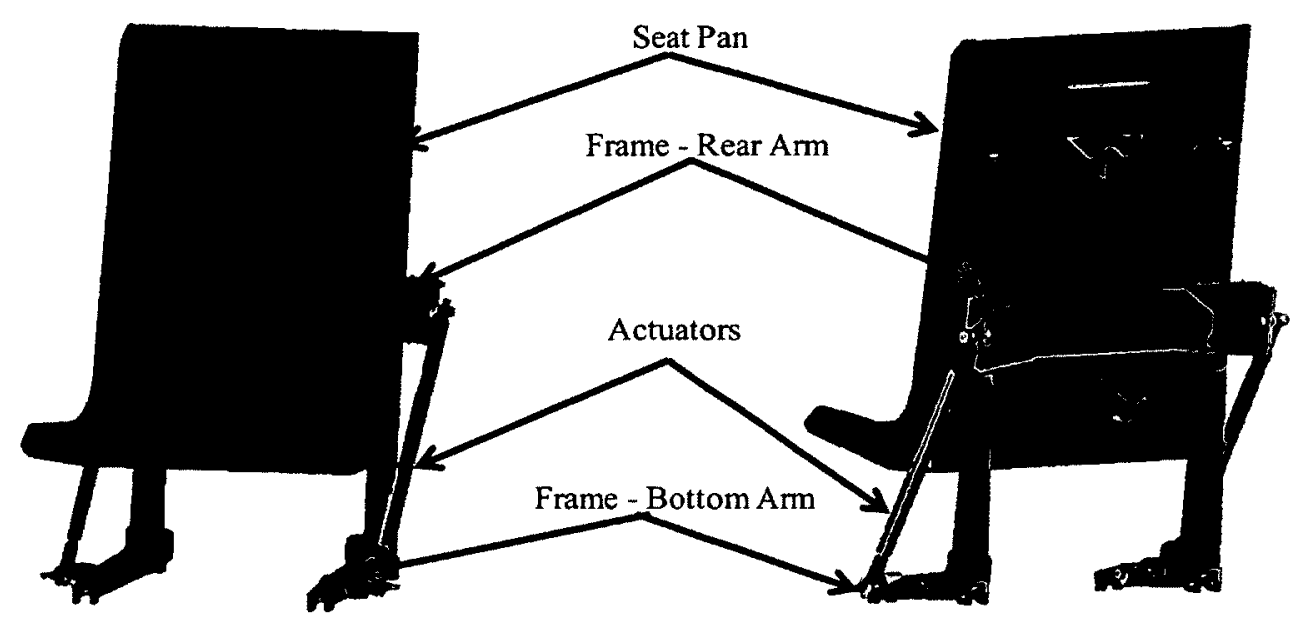

Figure 6.1: Alternate mechanical design of the adaptive seat mount

Alternately, the mechanical design of the actuator placement could be refined such that the piezoelectric stacked actuators are not connected to the seat pan to avoid interfering with the existing crash resistant mechanism in the Bell-412 seats. This could be achieved by placing the actuators between bottom and rear arms of the L-shaped seat frame as shown in Figure 6.1. This modification to the seat frame with additional active struts would also increase the bending stiffness of the seat to move the bending mode out of the range of major rotor harmonics while generating forces to counteract the forced vibration. However, in order to overcome the higher stiffness of the seat frame, the actuators will be required to generate higher forces compared to the implemented design. This higher force requirement could be satisfied by similar sized stacked piezoelectric 
actuators, which are inherently capable of providing large control forces. The actuator specifications could also be enhanced to improve the vibration suppression performance of the adaptive seat mount approach. Such refinements of the mechanical design will address the crashworthiness concerns and improve the vibration suppression performance to facilitate practical implementation of the adaptive mount methodology for helicopter seat application.

\subsubsection{Enhancement of the Biodynamic Seat Model}

The biodynamic lumped parameter model was developed as a simplified model to represent the dynamics of the seat structure as well as the human body for efficient vibration simulations. Although the cushion and the seat frame were modeled separately for this investigation, the model was only capable of handling linear properties for the cushion material. In general, cushion material properties are generally highly non-linear. Therefore, it is recommended to refine this biodynamic model to incorporate non-linear cushion properties. Such an enhanced lumped parameter biodynamic model will be an efficient tool to evaluate various seat cushion material configurations to mitigate aircrew vibration in helicopters without high computational burden.

\subsubsection{Helicopter Integration of the Actuation System}

Future studies should also be focused on qualifying the adaptive mount actuation approach for helicopter integration. Current investigation developed only a proof-ofconcept system for laboratory investigation and this system was not optimized for helicopter integration. Many aspects of the actuation system needed to be qualified for the helicopter application in consultation with airworthiness certification authorities and other relevant stake holders.

For example, the weight of the additional equipment required for the adaptive seat mount implementation will be a concern for any aircraft application, particularly for retrofitting existing aircraft. Therefore, further investigation should be carried out to minimize the weight of the complete system that includes the actuators, control computer, 
sensors as well as the power supply. As mentioned earlier, the power amplifier used in the current proof-of-concept development was not optimized for helicopter integration. Therefore, the power required to operate the actuation system must also be studied in detail because limited power is available in helicopters, especially for non-flight critical systems such as the adaptive seat mounts. In addition, the physical size of the actuators must be selected properly to ensure proper installation within the limited space around the seat. The size of the power amplifier is also a concern. Since high voltage power amplifiers are inherently bulky, investigating the use of alternate amplifier designs to reduce the volume would also be an advantage. Other qualification requirements of the system that must be addressed prior to helicopter integration include environmental considerations such as salt, moisture, temperature and flammability, as well as, electronic considerations such as electromagnetic interference with critical systems in the aircraft.

Addressing these system integration and airworthiness certification issues such as weight, power, size, and environmental considerations will make this novel adaptive seat mount approach based on active control technology significantly more attractive for both the military and commercial helicopter applications. Thereafter, it is recommended to integrate the complete adaptive seat mount system in a helicopter in order to perform flight tests to confirm the aircrew vibration reduction capability of this novel vibration attenuation system under the realistic operating conditions.

\subsubsection{Expanded Flight Test Program to Survey Cabin Environment}

The flight test performed on a Bell-412 helicopter during this investigation demonstrated that the helicopter aircrew is exposed to significantly high levels of vibration during flight. Analysis of the flight data based on the commonly used ISO 2631 standard to assess the effect of the whole body vibration exposure concluded the necessity to reduce the level of vibration experienced by helicopter aircrew. Although this data was sufficient for the current purpose of developing an adaptive seat mount concept, it is important to note that the vibration data analyzed for each flight condition was acquired by flying a single aircraft twice at a specified flight condition with a particular pilot and co-pilot. Therefore, it is recommended that more extensive flight tests be conducted by a 
larger population of pilots and co-pilots as well as by using several aircraft of the same type to obtain a more statistically significant set of flight data.

Furthermore, this flight test on a Bell-412 helicopter was required because the level of vibration exposure by the aircrew were not found in published literature. This lack of information prohibits the identification of potential health hazards for aircrew and development of suitable solutions. The growing demand for extended mission durations in the military and back-to-back flights in commercial aviation increases the potential health issues due to prolonged exposure to noise and vibration. Similar to vibration, high level of noise affects not only communication but more importantly prolonged exposure may lead to health hazards such as risk of heart attack, high blood pressure, hearing loss, loss of flight status or even disability. Therefore, it is recommended to conduct a comprehensive flight test program to survey the aircraft cabin environment to evaluate aircrew noise and vibration exposure levels. Such characterization effort utilizing stateof-the-art measurement techniques and statistically significant set of data samples could be used to determine permissible exposure time limits for the aircrew according to applicable technical standards and regulations such as ISO 2631. Such study could lead to establishment of guidelines and precautionary measures to minimize adverse health effects on aircrew.

\subsubsection{Airworthiness Certification of the Novel Cushion Material}

Although flight tests demonstrated that the Mitigator cushion manufactured with urethane material consistently reduced the aircrew vibration, the performance of this selected cushion configuration requires further validation through additional flight tests under varying conditions. The impact of varied aircrew weight as well as different flight conditions should be considered in order to validate the measured improvements for the majority of the aircrew population in most flight conditions. Despite the preliminary nature of this investigation, the results clearly demonstrated that the aircrew vibration levels can be effectively mitigated through proper optimization of the seat cushion impedance properties. The insertion of the Mitigator urethane cushion pad significantly reduced the $1 /$ rev peak while marginally reducing the $4 /$ rev peak as well. Although the 
modification of the cushion properties provided relatively lower vibration suppression performance compared to the modifications to the seat structure, it is a low cost solution with relatively less certification effort.

Therefore, it is recommended to investigate the integration of the Mitigator urethane pad with the original seat cushions in the Bell-412 helicopters as a near-term solution to reduce aircrew exposure to vibration. During the experimental flight test, it was possible to test the novel cushion material by placing it on the seat pan underneath the original cushion. However, practical implementation requires designing and fabricating a seat cushion integrated with the novel energy absorbing material that could be certified for aircraft application. Such a modified seat cushion dampens the amplification of $1 / \mathrm{rev}$ peak due resonance while maintaining the superior high frequency vibration suppression performance of the original cushion and matches the physical dimensions to retrofit the existing seat pan. This optimized seat cushion must be characterized through extensive shaker tests and the vibration suppression performance must be validated through a comprehensive flight test program under realistic operational conditions. In addition, other environmental tests such as the flammability, temperature, moisture, salt, et cetera must be conducted to qualify this novel cushion material to gain the required airworthiness certification.

Airworthiness certification of a retrofit seat cushion with superior vibration suppression qualities is a low cost and a near-term solution to mitigate undesirable occupational health hazards in helicopter aircrew due to high vibration exposure. Therefore, it is highly recommended to pursue the development and certification of this novel energy absorbing seat cushion system for the Bell-412 helicopter. 


\section{Appendix A}

\section{Bell-412 Aircrew Vibration Spectra from Flight Tests}

\section{A.1 Test Flight Conditions}

The test flight was conducted on November 27, 2007 using the NRC Bell-412 helicopter. The NRC Bell-412 helicopter is a specially configured test helicopter integrated with various flight sensors and an onboard data acquisition system. The data was acquired for a wide range of flight conditions such as ground, hover, translational lift, forward flight at $60 \mathrm{kts}, 120 \mathrm{kts}$ and $140 \mathrm{kts}$, in order to identify the variation in vibration profile during a typical flight. In general, the wind speed was $8 \mathrm{kts}$ from 290 degrees. The tested pilot represented a 50 percentile adult male and the co-pilot represented a 95 percentile adult male in terms of height and weight.

\section{A.2 Sensor Instrumentation System}

The data acquisition system was configured for simultaneous recording of the accelerometer channels at a sampling rate of $2 \mathrm{kHz}$. Accelerometers installed on the structure were bonded with epoxy while the accelerometers that were placed on the aircrew were affixed with wax to facilitate ease of removal. All miniature accelerometers were calibrated to verify accuracy and ensure reliability. The time domain data were recorded digitally and simultaneously analyzed in the frequency domain to reveal the real-time vibration spectrum from each accelerometer using the onboard laptop computer. 


\section{A.3 Spectral Data Analysis Parameters}

A minimum of two data points were taken for each flight condition to verify repeatability. Each data point lasted 1 minute while the aircraft maintained the specified flight condition. The spectra were analyzed using a frequency resolution of $0.25 \mathrm{~Hz}$ with a Flat-Top windowing function and 15 linear averages.

\section{A.4 Measurement Locations and Directions}

The measurement locations included the floor under the seat, bottom of the seat, top of the cushion, aircrew shoulder, helmet, and the top of the instrument panel. The floor, seat and the helmet locations for the pilot included accelerometers in all 3 directions while those locations for the co-pilot measured only in $\mathrm{X} \& \mathrm{Z}$ directions. The vibration at remaining locations was measured only in the $Z$ direction. Table A.1 summarizes the figures that shows all measured spectra for each flight conditions with the corresponding test run.

Table A.1: Vibration spectra with corresponding flight conditions for each test run

\begin{tabular}{|c|c|c|}
\hline Figure No. & Flight Condition & Run No \\
\hline Figure A.1 & Ground Run (1) & 1 \\
\hline Figure A.2 & $25 \mathrm{ft} \mathrm{Hover} \mathrm{(1)}$ & 2 \\
\hline Figure A.3 & Trans. Lift (1) & 3 \\
\hline Figure A.4 & $60 \mathrm{kts}$ Climb (1) & 5 \\
\hline Figure A.5 & $60 \mathrm{kts}$ Level (1) & 6 \\
\hline Figure A.6 & $100 \mathrm{kts}$ Level (1) & 7 \\
\hline Figure A.7 & $120 \mathrm{kts}$ Level (1) & 8 \\
\hline Figure A.8 & $140 \mathrm{kts}$ Desc. (1) & 9 \\
\hline Figure A.9 & $60 \mathrm{kts}$ Climb (2) & 11 \\
\hline Figure A.10 & $120 \mathrm{kts}$ Level (2) & 12 \\
\hline Figure A.11 & $60 \mathrm{kts}$ Level (2) & 13 \\
\hline Figure A.12 & $100 \mathrm{kts}$ Level (2) & 14 \\
\hline Figure A.13 & $140 \mathrm{kts}$ Level (1) & 15 \\
\hline Figure A.14 & Trans. Lift (2) & 17 \\
\hline Figure A.15 & $25 \mathrm{ft} \mathrm{Hover} \mathrm{(2)}$ & 18 \\
\hline Figure A.16 & Ground Run (2) & 19 \\
\hline
\end{tabular}



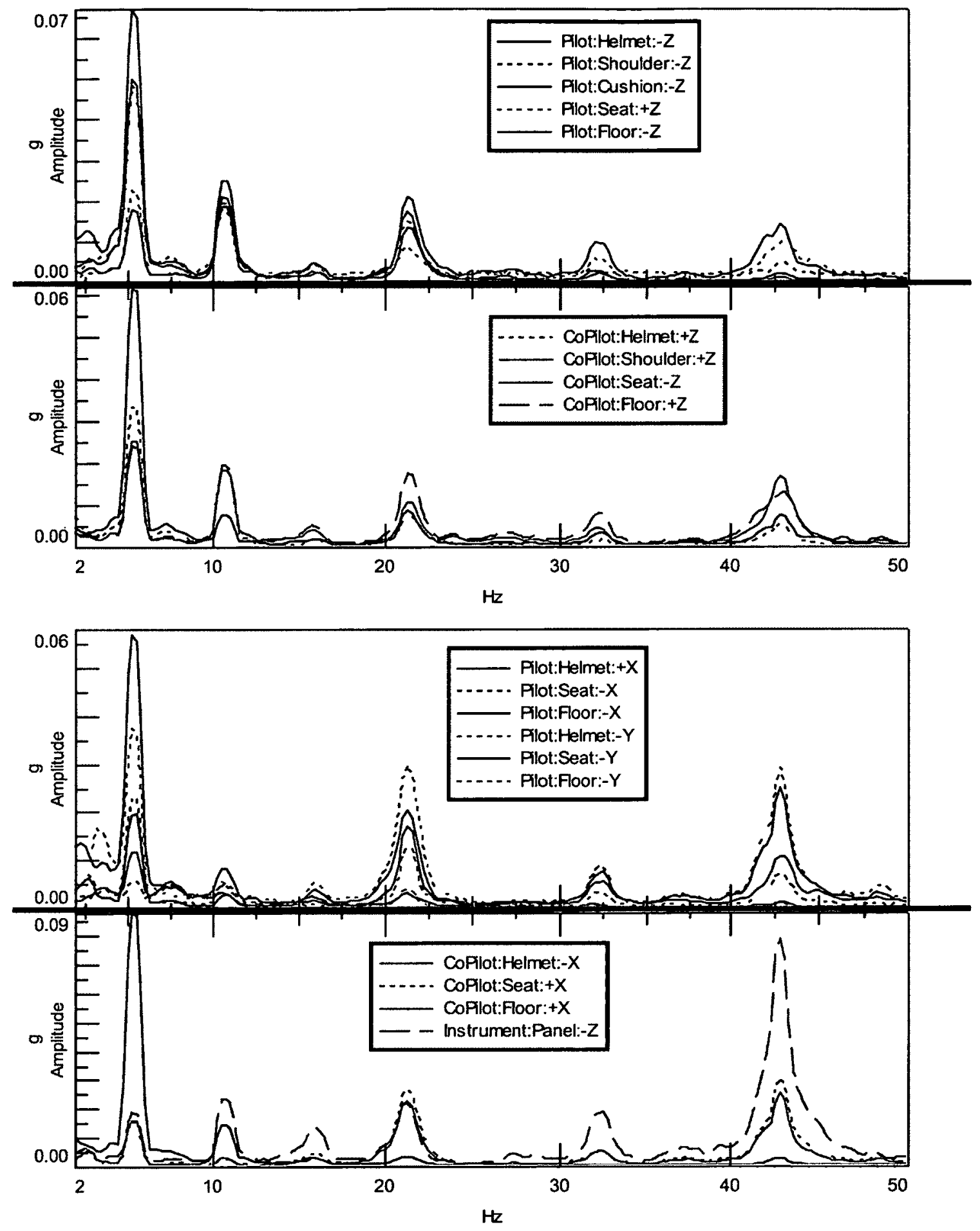

Figure A.1: Vibration Spectra from Run No. 1 for flight condition ground run (1) 

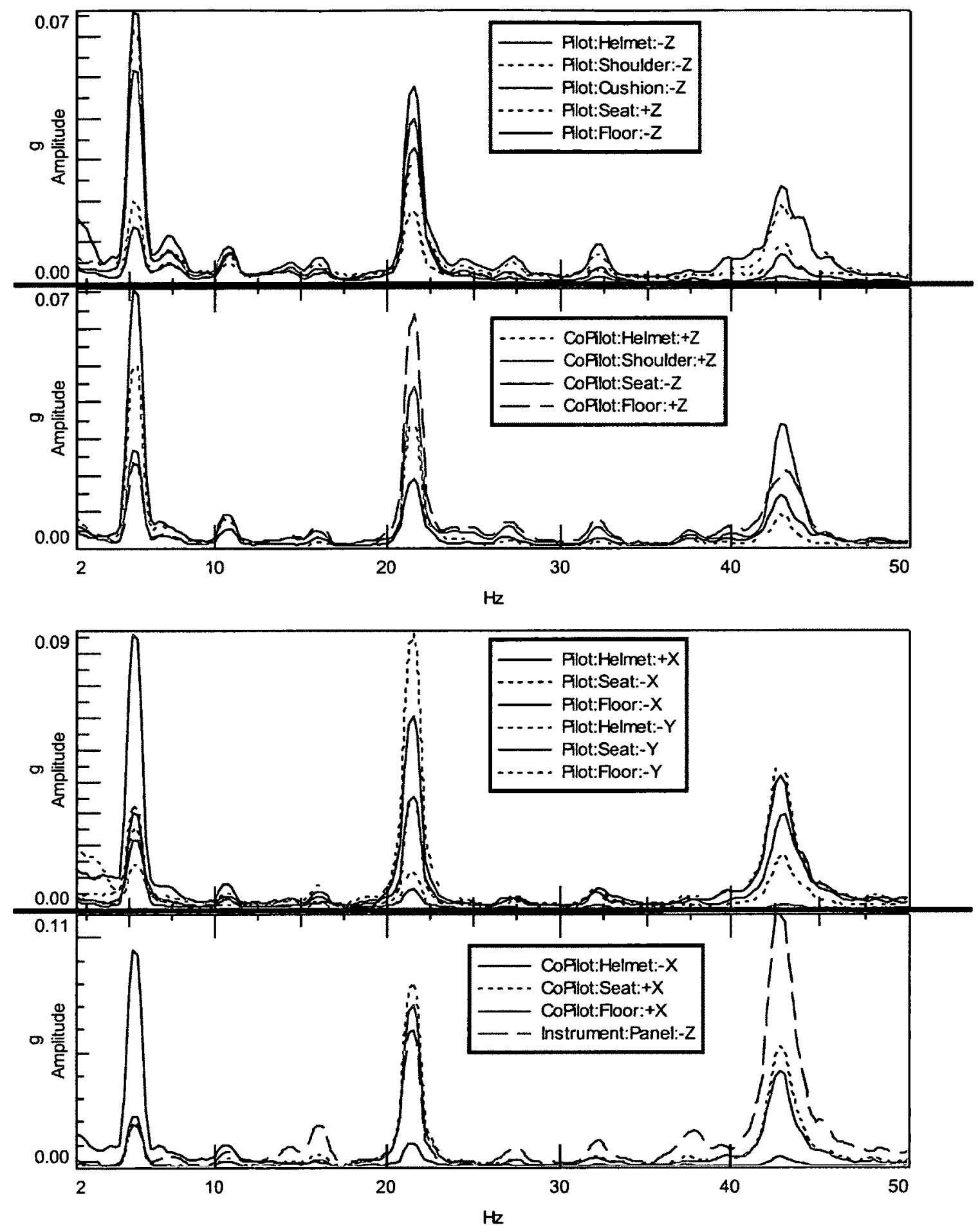

Figure A.2: Vibration Spectra from Run No. 2 for flight condition $25 \mathrm{ft}$ hover (1) 

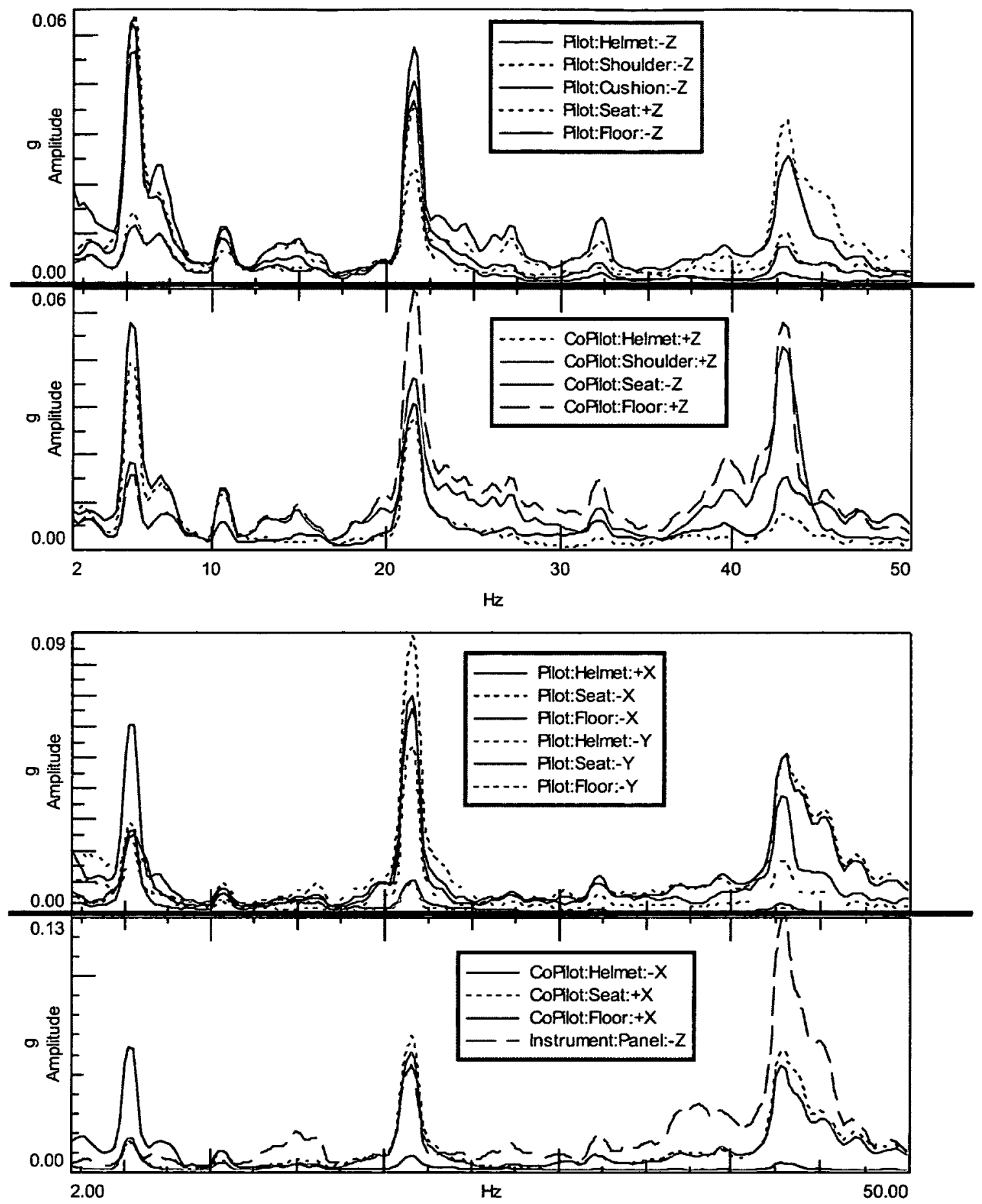

Figure A.3: Vibration Spectra from Run No. 3 for flight condition trans. lift (1) 

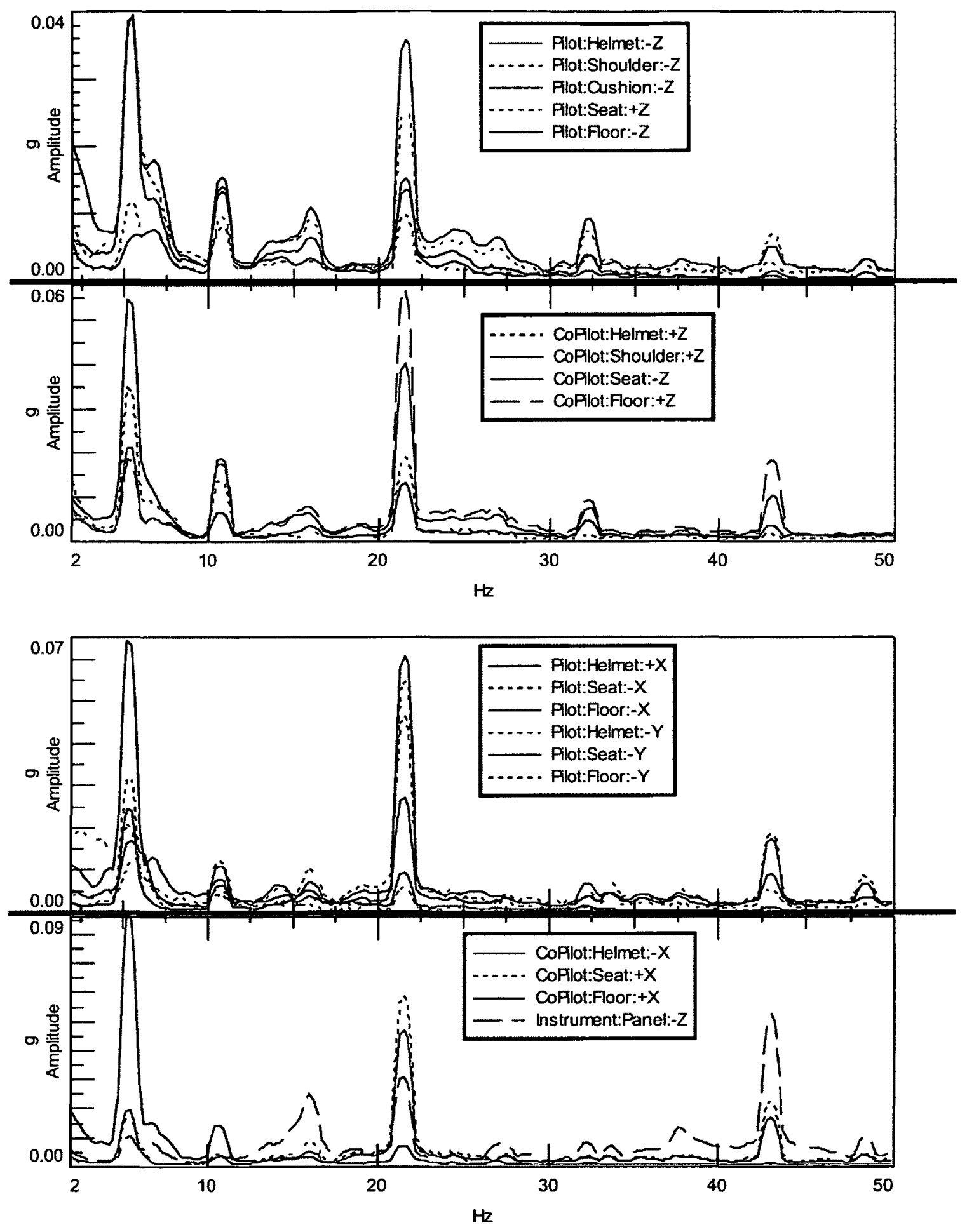

Figure A.4: Vibration Spectra from Run No. 5 for flight condition 60 kts climb (1) 

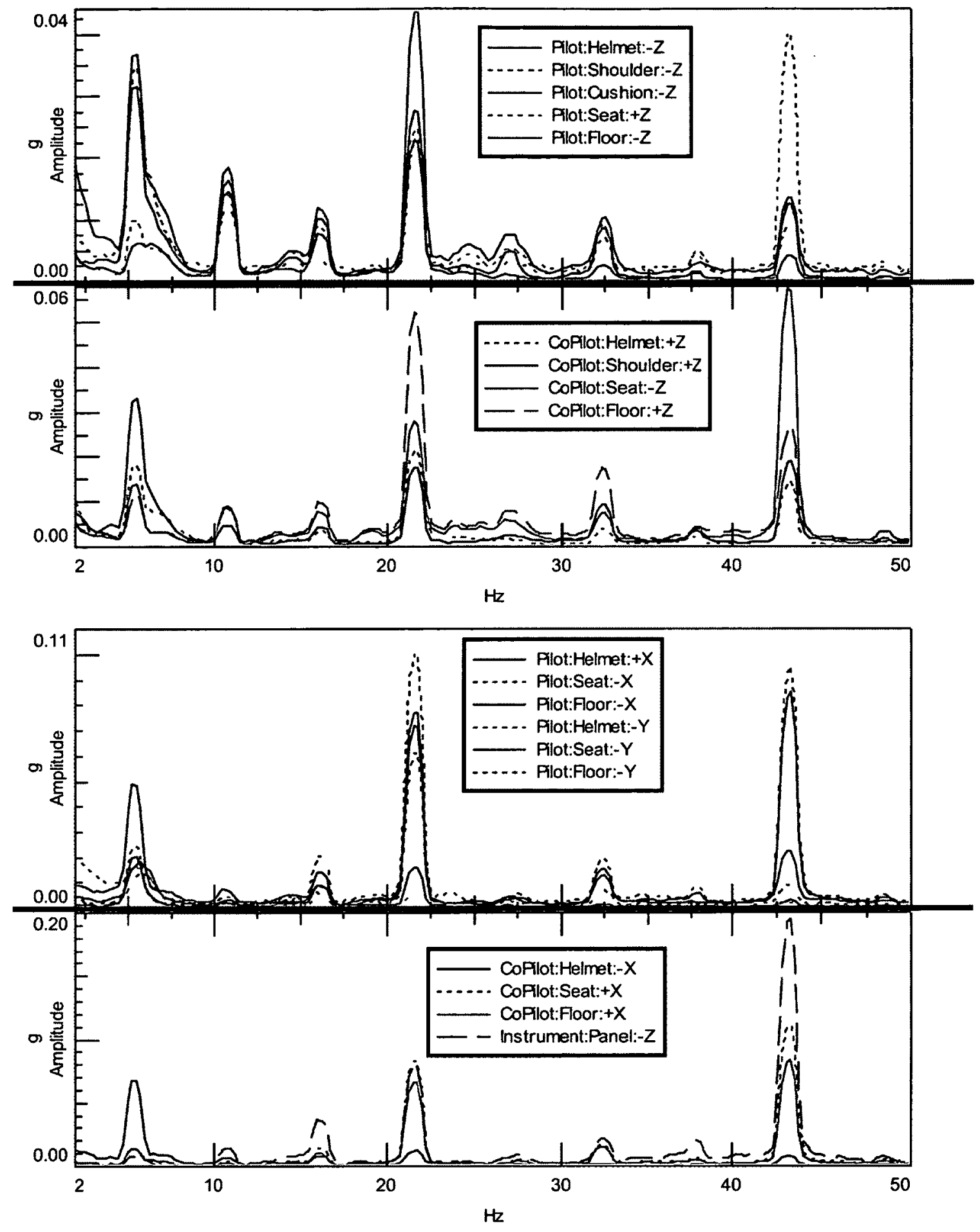

Figure A.5: Vibration Spectra from Run No. 6 for flight condition 60 kts level (1) 

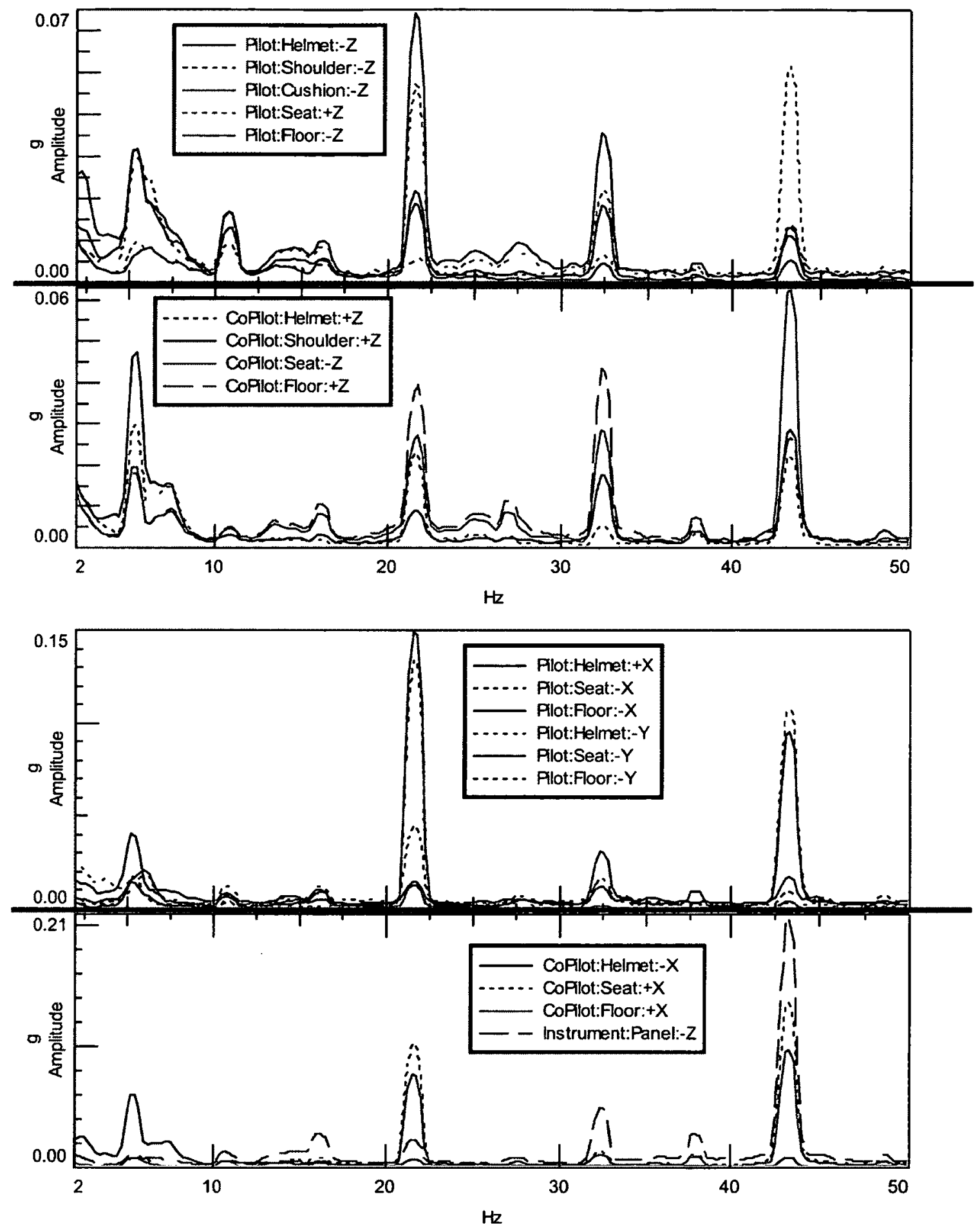

Figure A.6: Vibration Spectra from Run No. 7 for flight condition 100 kts level (1) 

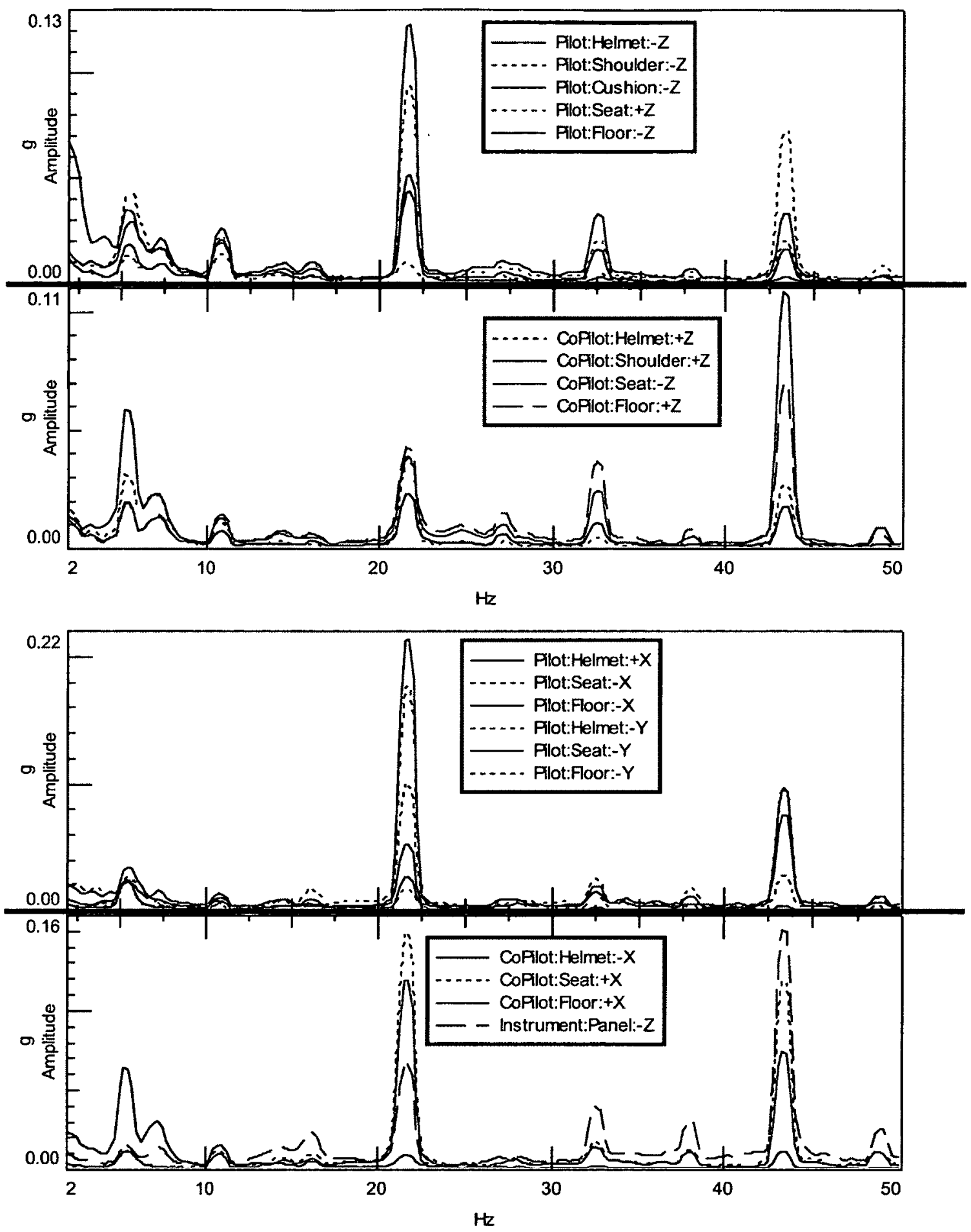

Figure A.7: Vibration Spectra from Run No. 8 for flight condition 120 kts level (1) 

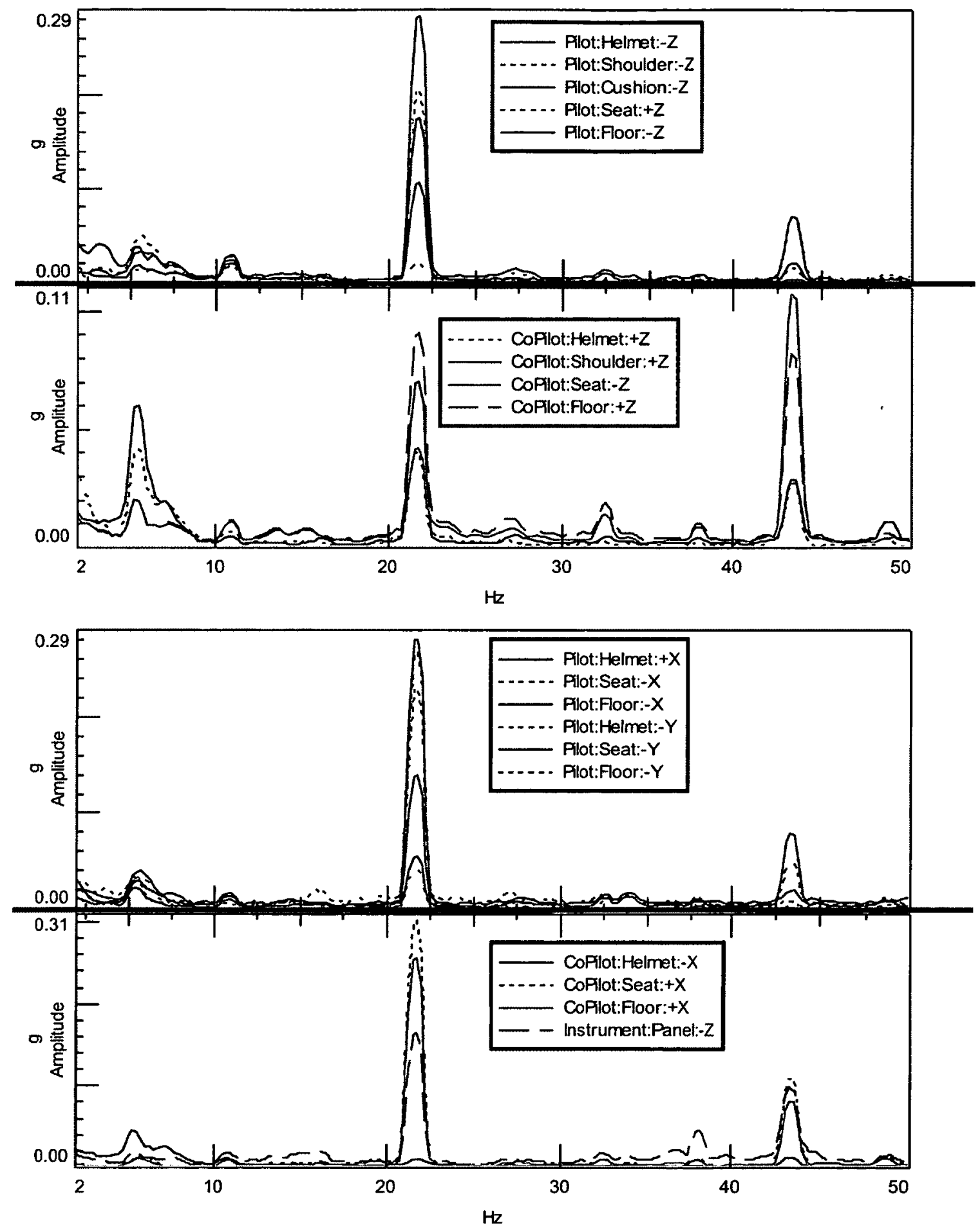

Figure A.8: Vibration Spectra from Run No. 9 for flight condition 140 kts descent (1) 

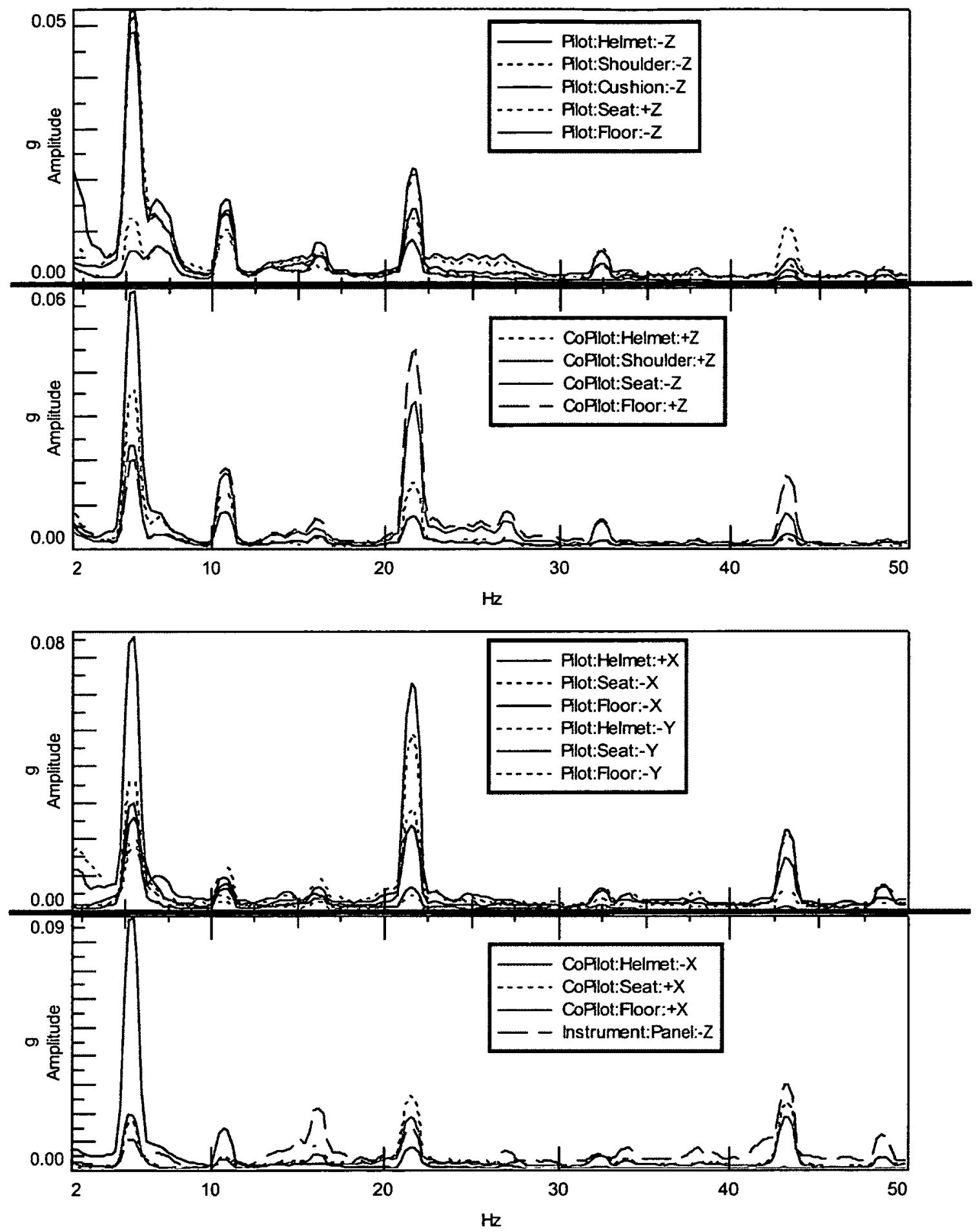

Figure A.9: Vibration Spectra from Run No. 11 for flight condition 60 kts climb (2) 

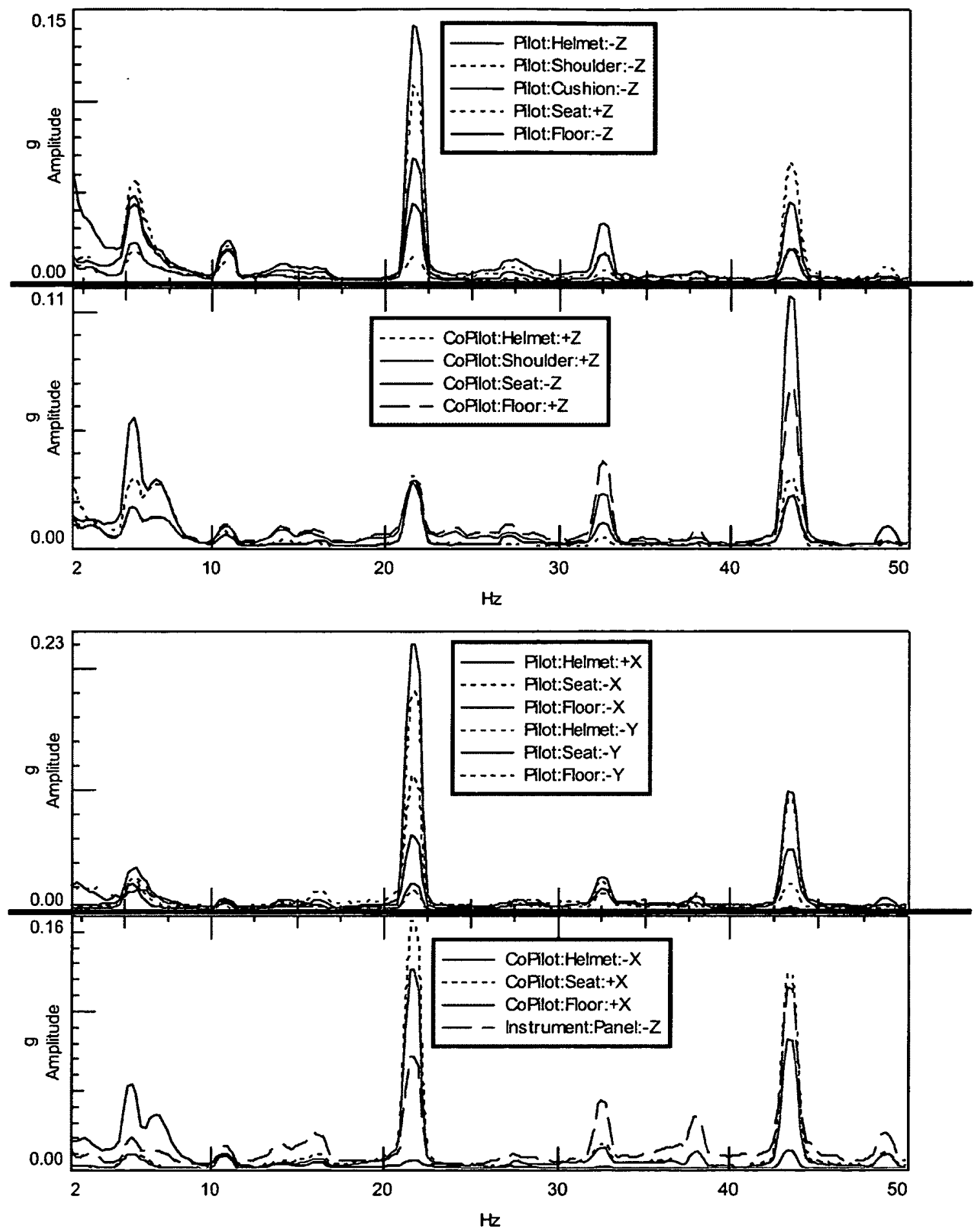

Figure A.10: Vibration Spectra from Run No. 12 for flight condition 120 kts level (2) 

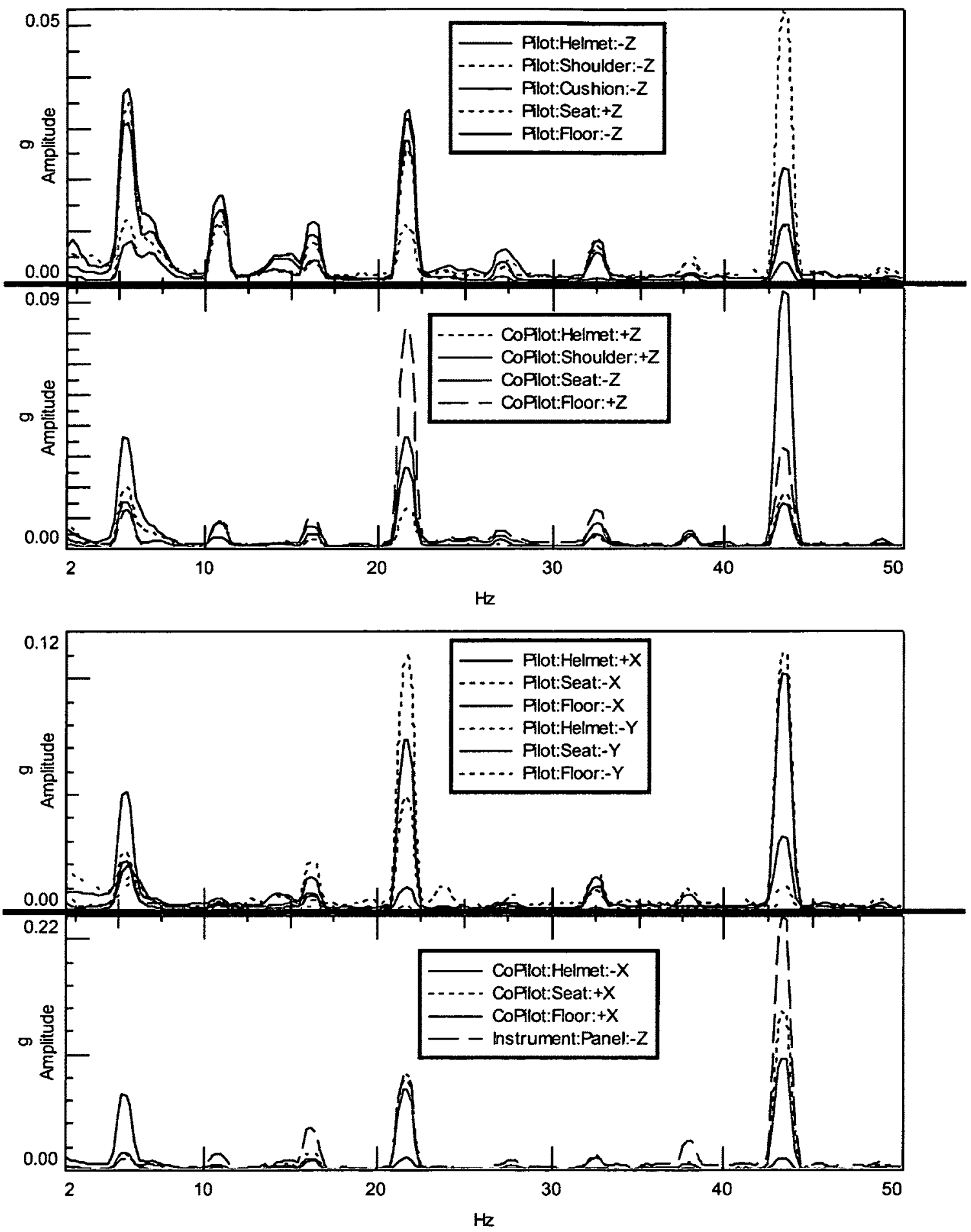

Figure A.11: Vibration Spectra from Run No. 13 for flight condition 60 kts level (2) 

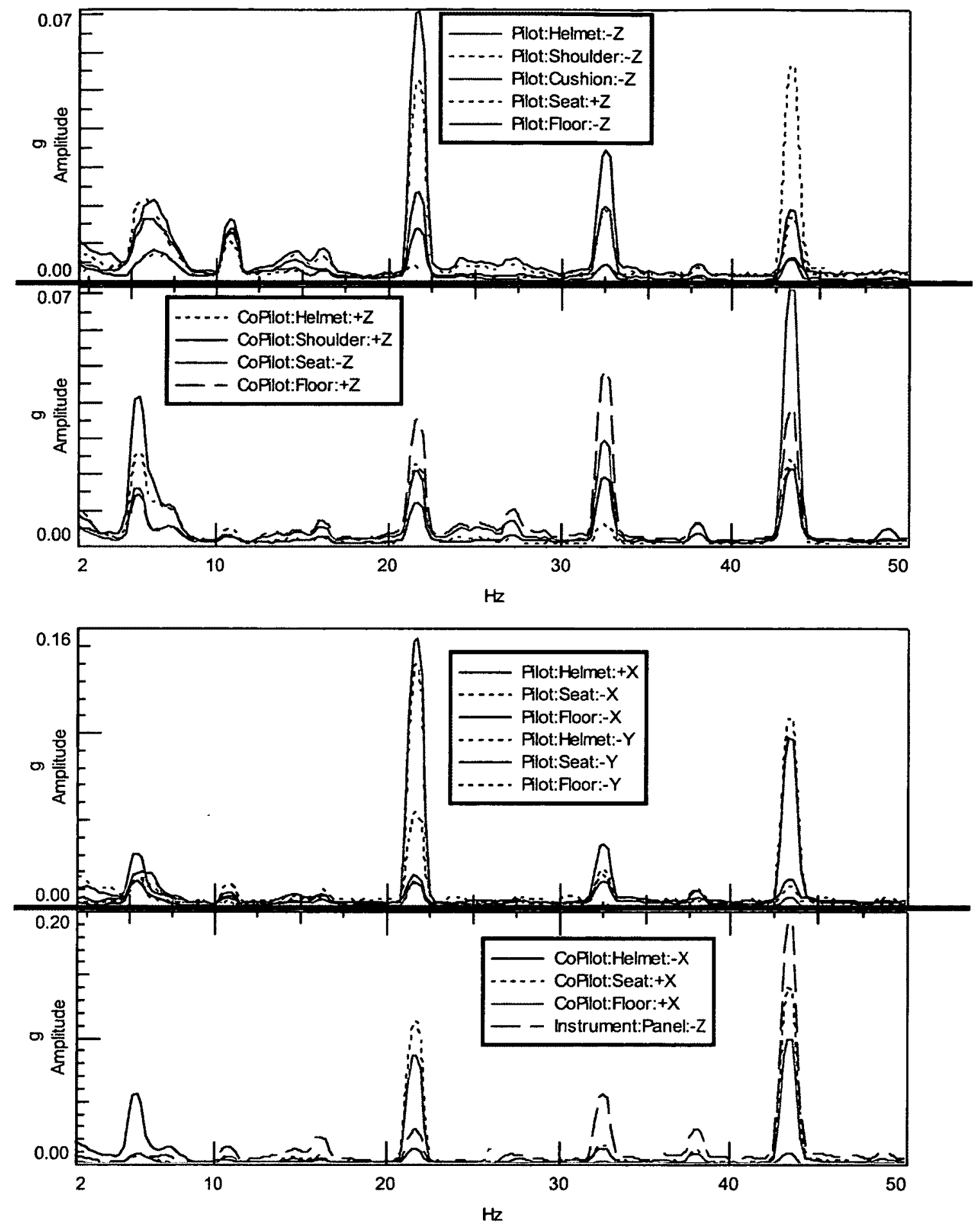

Figure A.12: Vibration spectra from Run No. 14 for flight condition 100 kts level (2) 

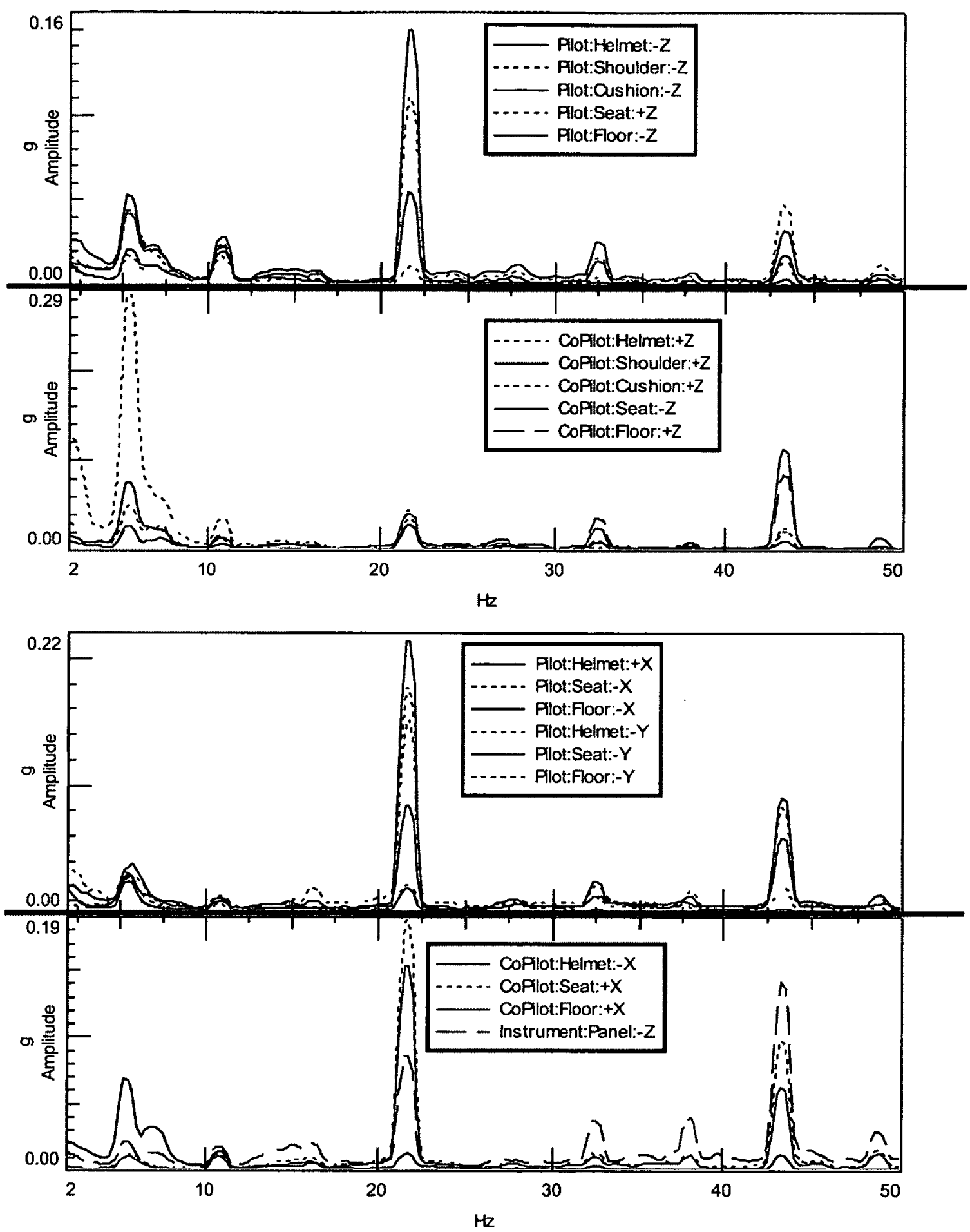

Figure A.13: Vibration spectra from Run No. 15 for flight condition $140 \mathrm{kts}$ level (1) 

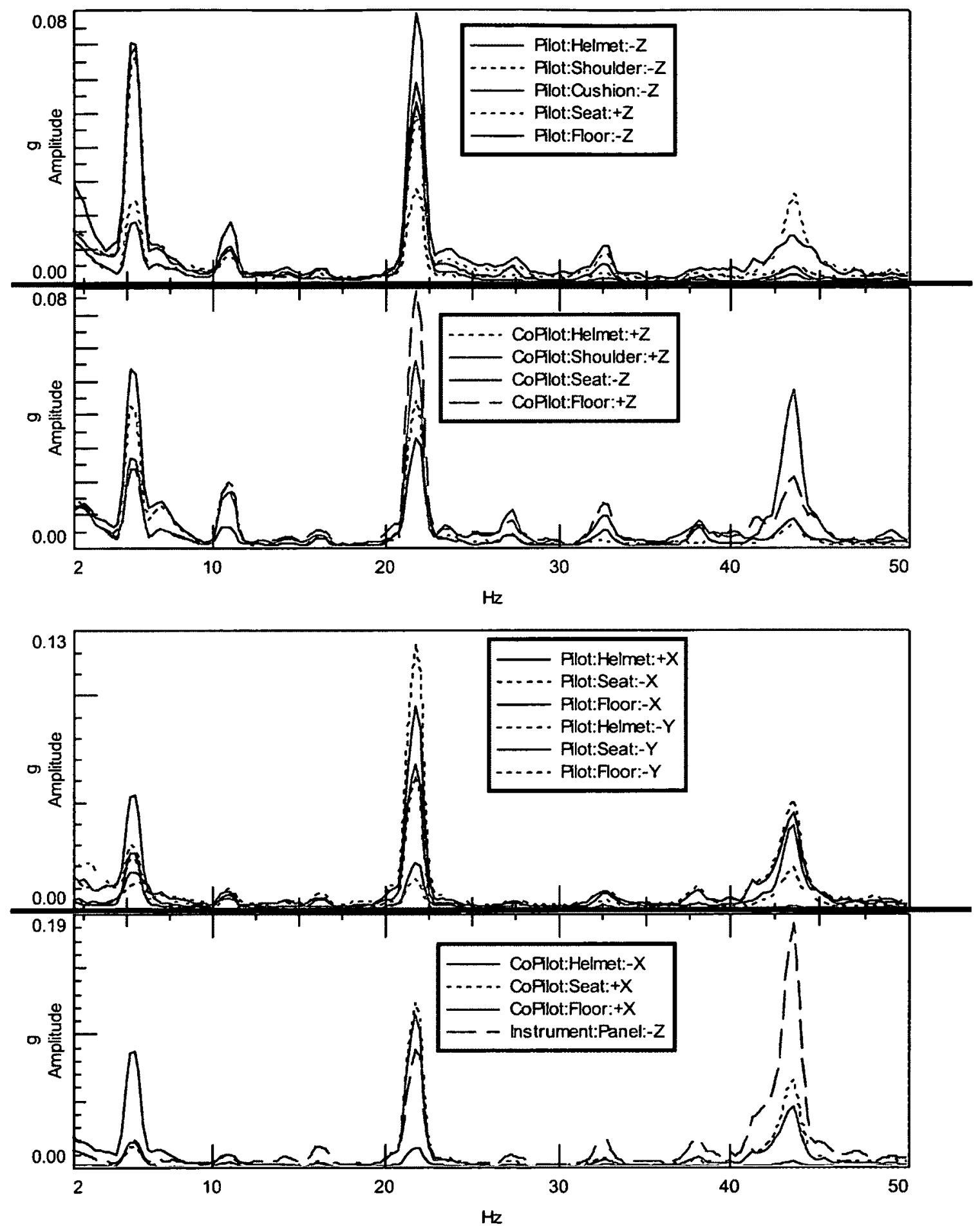

Figure A.14: Vibration spectra from Run No. 17 for flight condition trans. lift (2) 

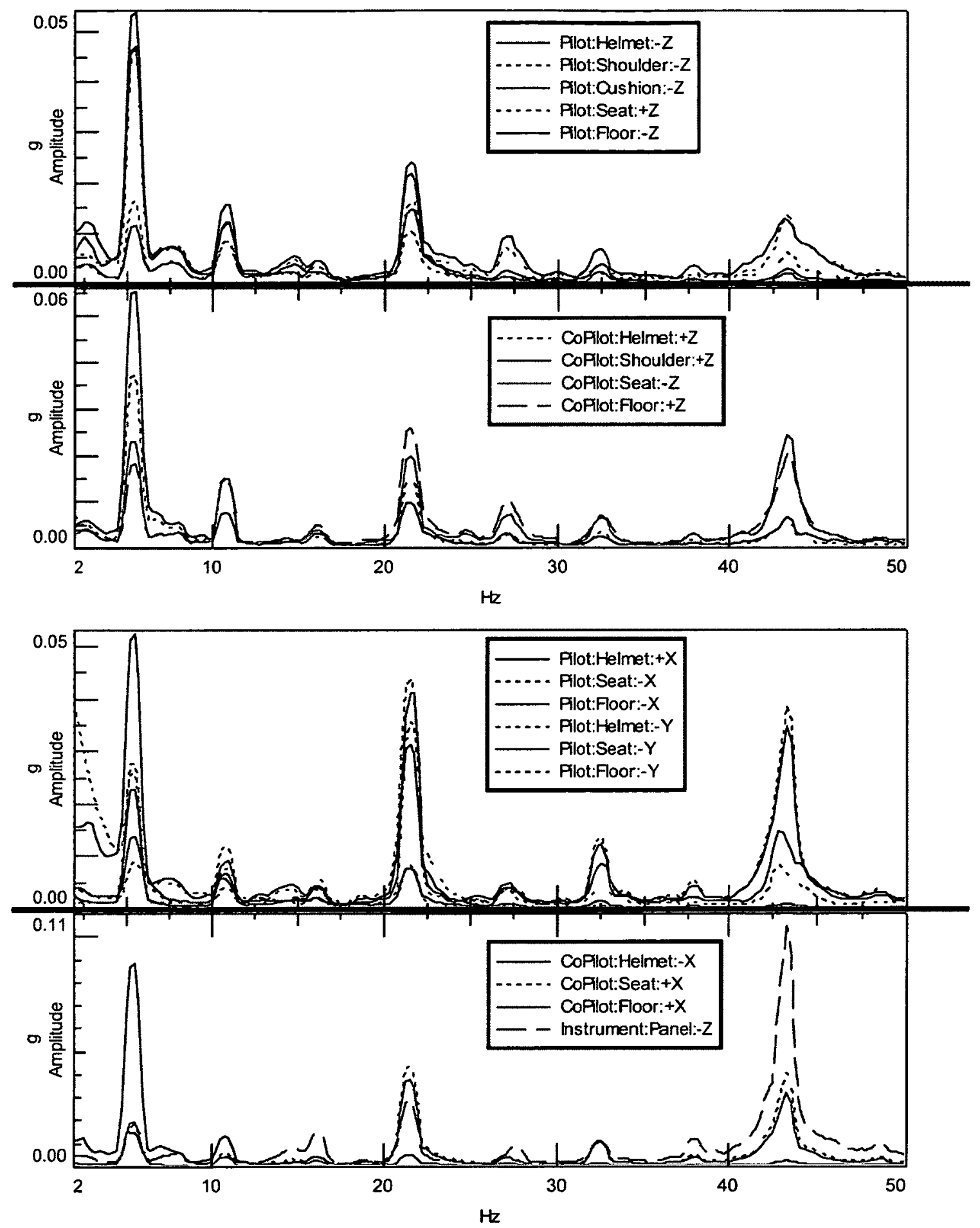

Figure A.15: Vibration spectra from Run No. 18 for flight condition $25 \mathrm{ft}$ hover (2) 

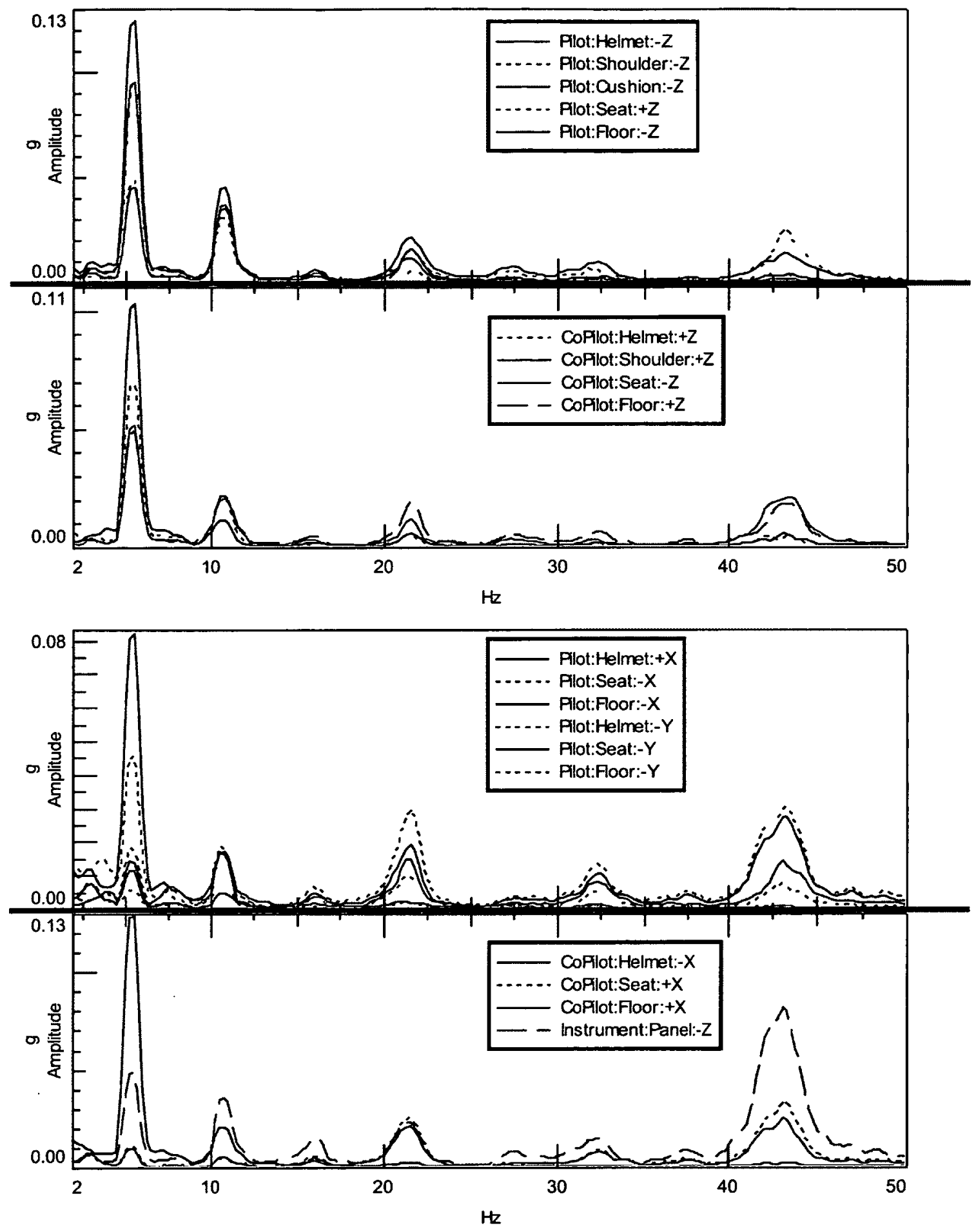

Figure A.16: Vibration spectra from Run No. 19 for flight condition ground run (2) 


\section{Appendix B}

\section{State Space Formulation of the Biodynamic Model}

\section{B.1 Definition of State-Space Form}

The equations of motion of the biodynamic model were transformed into the State Space form for efficient simulation. The state variables define the initial, current and future response of a system. State-space equations is expressed as

$$
\begin{aligned}
& \dot{\mathbf{x}}=\mathbf{A x}+\mathbf{B u} \\
& \mathbf{y}=\mathbf{C} \mathbf{x}+\mathbf{D u}
\end{aligned}
$$

where, $\mathbf{x}$ represents the $n$-dimensional state vector, $\mathbf{y}$ the output vector, $\mathbf{u}$ the control input, $\mathbf{A}$ the system matrix, $\mathbf{B}$ the actuator influence matrix, $\mathbf{C}$ provides the sensor influence matrix and $\mathbf{D}$ the direct transmission matrix. Usually, $\mathbf{D}$ is considered as zero except for feed-forward systems.

\section{B.2 Derived State-Space Matrices}

The transformation of the variables produced following $\mathrm{A}, \mathrm{B}, \mathrm{C}$ and $\mathrm{D}$ matrices of the State Space model. The matrix $\mathrm{C}$ was constructed to observe the displacement of desired component of the biodynamic model, primarily the vibration of the aircrew head. 
$\mathrm{B}=\left(\begin{array}{cc}0 & 0 \\ 0 & 0 \\ 0 & 0 \\ 0 & 0 \\ 0 & 0 \\ 0 & 0 \\ \frac{k_{F}}{m_{F}} & \frac{c_{F}}{m_{F}} \\ 0 & 0 \\ 0 & 0 \\ 0 & 0 \\ 0 & 0 \\ 0 & 0\end{array}\right)$

$\mathrm{C}=\left(\begin{array}{llllllllllll}1 & 0 & 0 & 0 & 0 & 0 & 0 & 0 & 0 & 0 & 0 & 0 \\ 0 & 1 & 0 & 0 & 0 & 0 & 0 & 0 & 0 & 0 & 0 & 0 \\ 0 & 0 & 0 & 0 & 0 & 1 & 0 & 0 & 0 & 0 & 0 & 0\end{array}\right)$

$\mathrm{D}=\left(\begin{array}{ll}0 & 0 \\ 0 & 0 \\ 0 & 0\end{array}\right)$ 


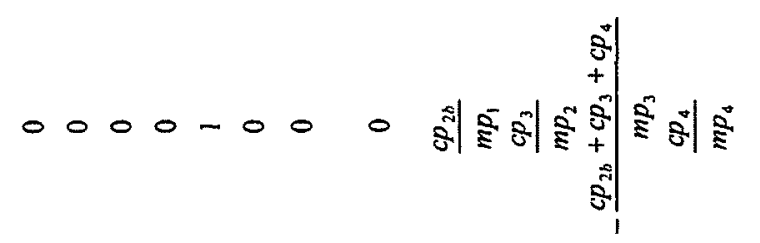

$000-0000000$

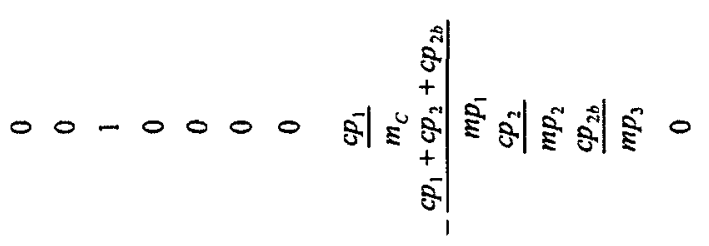

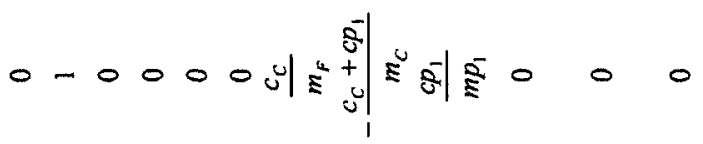

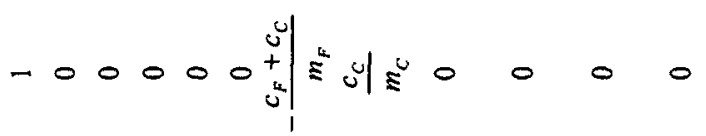

0000000000000

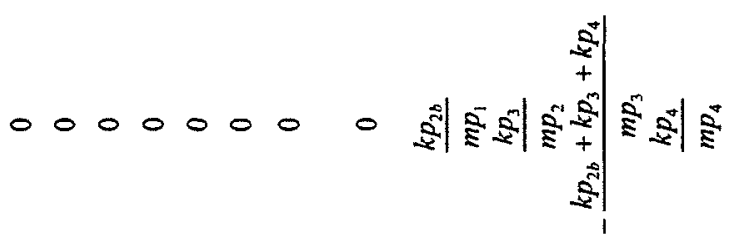

000000000

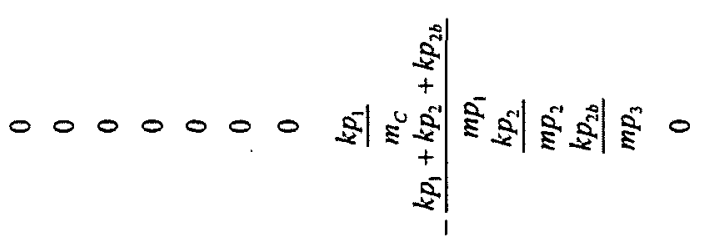

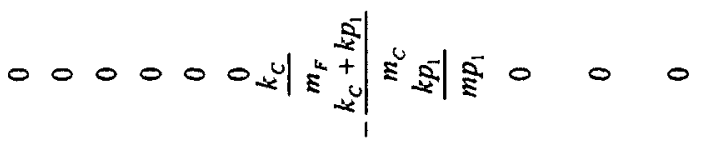

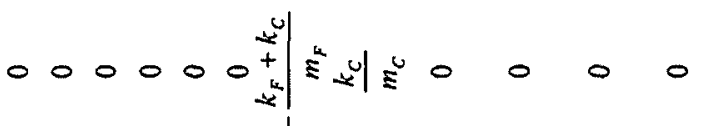


INTENDED TO LEAVE BLANK 


\section{References}

[1] Smith, D., 2002, "Characterizing the Effects of Airborne Vibration on Human Body Vibration Response," Journal of Aviation, Space, and Environmental Medicine, 73(3), 2002, pp. 36-45.

[2] Castelo-Branco, N. A. A., and Rodriguez, E., 1999, "The Vibroacoustic Disease An Emerging Pathology," Journal of Aviation, Space, and Environmental Medicine, 70(1), pp. A1-A6.

[3] Adams J., 2004, "Results of NVG-Induced Neck Strain Questionnaire Study in CH146 Griffon Aircrew," Report No.: TR2004-153, Defence Research and Development Canada, Toronto, Canada.

[4] Shanahan, D.F. and Reading, T.E., 1984, "Helicopter Pilot Back Pain: A Preliminary Study," Journal of Aviation, Space, and Environmental Medicine, 55(2), pp. 117121.

[5] Thomae M. K., Porteus, J. E., Brock, J. R., Allen, G. D., Heller, R. F., 1998, “Back Pain in Australian Military Helicopter Pilots: A Preliminary Study." Journal of Aviation, Space, and Environmental Medicine, 69(5): pp. 468-473.

[6] Ang, B., Harms-Ringdahl, K., 2006, "Neck pain and related disability in helicopter pilots: a survey of prevalence and risk factors," Journal of Aviation, Space, and Environmental Medicine, 77(7), pp. 713-719.

[7] Thomae, M. K., Porteus, J. E., Brock, J. R., Allen, G. D., Heller, R. F., 1998, "Back pain in Australian Military Helicopter Pilots: A Preliminary Study," Journal of Aviation, Space, and Environmental Medicine, 69(5), pp. 468-473.

[8] Harrison M. F, Neary, J.P., Albert, W.J., Croll, J. C., 2011, "Neck Pain and Muscle Function in a Population of CH-146 Helicopter Aircrew," Journal of Aviation, Space, and Environmental Medicine, 82(12), pp. 1125-1130. 
[9] United States Department of Defense, 1981, "Seats, Helicopter Cabin, Crashworthy, General Specification," MIL-S-85510(AS).

[10] United States Department of Defense, 1986, "Seat System: Crash-Resistant, NonEjection, Aircrew, General Specification" MIL-S-58095A (AV).

[11] Kretz, M., and Larche, M., 1980, "Future of Helicopter Rotor Control," Vertica, 4(1) pp. 13-22.

[12] Landgrebe, A. J., and Davis, M. W., 1984, "Analysis of Potential Helicopter Vibration Reduction Concepts," AHS Decennial Specialists' Meeting on Rotorcraft Dynamics, Moffett Field, CA.

[13] Bielawa, R. L., 1992, Rotary Wing Structural Dynamics and Aeroelasticity, American Institute for Aeronautics and Astronautics, Reston, VA.

[14] Amer, K. B., and Neff, J. R., 1974, "Vertical-Plane Pendulum Absorbers for Minimizing Helicopter Vibratory Loads, "Journal of the American helicopter Society, 19(4), pp. 44-48.

[15] Braun, D., 1984, "Ground and Flight Tests of a Passive Rotor Isolation System for Helicopter Vibration Reduction," Vertica, 8(1), pp. 1-14.

[16] Millot, T., and Friedman P., 1992, "Vibration Reduction in Helicopter Rotors Using Active Control Surface Located on the Blade", , Paper No. 92-2451, $33^{\text {rd }}$ AIAA Structures, Structural Dynamics and Materials Conference, Dallas, TX.

[17] Payne, P. R., 1958, "Higher Harmonic Rotor Control: The Possibilities of Third and Higher Harmonic Feathering for Delaying the Stall Limit in Helicopters," Aircraft Engineering and Aerospace Technology, 30(8), pp. 222-226.

[18] Lemnios, A. Z., Nettles, W. E., and Howes, H. E., 1976, "Full Scale Wind Tunnel Tests of a Controllable Twist Rotor," Proceedings of a Symposium on Rotor Technology, American Helicopter Society. Alexandria, VA. 
[19] McCloud, J. L., and Weisbrich A. L., 1978, "Wind-Tunnel Test Results of a FullScale Multi-Cyclic Controllable Twist Rotor," $34^{\text {th }}$ Annual Forum of the American Helicopter Society, Washington, DC.

[20] Shaw, J., Albion, N., Hanker, E. J., and Teal, R. S., 1985, "Higher Harmonic Control: Wind Tunnel Demonstration of Fully Effective Vibratory Hub Force Suppression," Journal of the American Helicopter Society, 34(1), pp. 14-25.

[21] Nguyen, K. and Chopra, I., 1992, "Effect of Higher Harmonic Control on Rotor Performance and Control Loads," Journal of Aircraft, 29(3), pp. 336-342.

[22] Walsh, D. M., 1986, "Flight Tests of an Open Loop Higher Harmonic Control system of an S-76A Helicopter," $42^{\text {nd }}$ Annual Forum of the American Helicopter Society, Washington, DC. pp. 831-843.

[23] Kretz, M., 1976, “Research in Multicyclic and Active Control of Rotary Wings," Vertica, 1(2), pp. 95-105.

[24] Ham, N. D., 1987, "Helicopter Individual-Blade-Control Research at MIT 19771985," Vertica, 11(1/2), pp. 109-122.

[25] Jacklin, S. A., Blass, A., Swanson, S. M., and Teves, D., 1995, "Second Test of a Helicopter Individual Blade Control System in the NASA Ames 40 - by 80 -foot Wind Tunnel," $2^{\text {nd }}$ International Aeromechanics Specialists' Conference, Bridgeport, CT, pp. 7.9-7.26.

[26] Kube, R., Van Der Wall, B. G., 1999, "IBC Effects on BVI noise and Vibrations -A Combined numerical and Experimental Investigation," $55^{\text {th }}$ International Annual Forum of the American Helicopter Society, Montreal, Canada, pp. 2282-2291.

[27] Mueller, M., Arnold U. T. P., and Morbitzer, D., 1999, "On the Importance and Effectiveness of $2 /$ rev IBC for Noise, Vibration and Pitch Link Load Reduction," $25^{\text {th }}$ European Rotorcraft Forum, Rome, Italy.

[28] Nitzsche, F., Feszty, D., Waechter, D., Bianchi, E., Voutsinas, S., Gennaretti, M., Coppotelli, G., Ghiringhelli, G.L., 2005, “The SHARCS Project: Smart Hybrid 
Active Rotor Control System for Noise and Vibration Attenuation of Helicopter Rotor Blades," Paper No. 052, $31^{\text {st }}$ European Rotorcraft Forum, Florence, Italy.

[29] Mander, A., Feszty, D., Nitzsche, F., 2008, “Active Pitch Link Actuator for Impedance Control of Helicopter Vibration", Paper No. 080170, $64^{\text {th }}$ Annual Forum of the American Helicopter Society, Montreal, QC, Canada.

[30] Nitzsche, F., Grewal, A. and Zimcik, D. G., 1999, "Structural Component Having Means for Actively Varying its Stiffness to Control Vibrations" US patent $5,973,440$.

[31] Oxley, G., Nitzsche, F. and Feszty, D., 2009, "Smart Spring Control of Vibration on Helicopter Rotor Blades," Journal of Aircraft, 46(2), pp. 692-696.

[32] Nitzsche, F. et al, 2012, "Developments on the Hybrid Stiffness - Flow Control Active System for Rotor Vibration Attenuation Project - SHARCS," Paper 010, $38^{\text {th }}$ European Rotorcraft Forum, Amsterdam, The Netherlands.

[33] Straub, Friedrich K.; Kennedy, Dennis K.; Stemple, Alan D.; Anand, V. R.; Birchette, Terry S., 2004, "Development and Whirl Tower Test of the SMART Active Flap Rotor", Smart Structures and Materials 2004: Industrial and Commercial Applications of Smart Structures Technologies. Anderson, E. H., Ed., Proceedings of the SPIE, Vol. 5388, pp. 202-212.

[34] Chopra, I., Copp, P. 2008, "Continued Development of a Mach Scale Swashplateless Rotor with Integrated Trailing Edge Flaps", $64^{\text {th }}$ Annual Forum of the American Helicopter Society, Montreal, QC, Canada.

[35] Friedmann P.P., 2005, "Vibration and Noise Reduction Using Actively Controlled Flaps-Their Evolution and Potential for Improving Rotorcraft Technology", Paper No. ISF-079, IFASD Conference, Munich, Germany.

[36] Crozier, P., Leconte, P., Delrieux, Y., Gimonet, B., Le Pape, A., Des Rochettes, H., M., 2006, "Wind-Tunnel Tests of a Helicopter Rotor with Active Flaps", $32^{\text {nd }}$ European Rotorcraft Forum, Maastricht, The Netherlands, Vol. 1, pp 235-250. 
[37] Bernhard, A. and Chopra, I., 1999, "Trailing Edge Flap Activated by a PiezoInduced Bending-Torsion Coupled Beam," Journal of the American Helicopter Society, 44(1), pp. 3-15.

[38] Centolanza, L. R. and Smith, E. C., 2000, "Design and Experimental Testing of an Induced-Shear Piezoelectric Actuator for Rotor Blade Trailing Edge Flaps," $41^{s t}$ AlAA Structures, Structural Dynamics, and Materials Conference, Atlanta, GA.

[39] Prechtl, E. F., Hall, S. R., 1998, “An X-Frame Actuator Servo-Flap Actuation System for Rotor Control,”, Smart Structures and Materials 1998: Smart Structures and Integrated Systems, Regelbrugge; M. E., Ed., Proceedings of the SPIE, Vol. 3329, pp. 309-320.

[40] Fenn, R. C., Downer, J. R., Bushko, D. A., Gondhalekar, V., and Ham, N. D., 1996, “Terfenol-D Driven Flaps for Helicopter Vibration Reduction," Journal of Smart Materials and Structures, 5(1), pp. 49-57.

[41] Bothwell, C. M., Chandra, R., and Chopra, I., 1995, "Torsional Actuation with Extension-Torsion Composite Coupling and a Magnetostrictive Actuator," AIAA Journal 33(4), pp. 723-729.

[42] Millott, T. A. and Friedmann, P. P., 1994, "Vibration Reduction in Hingeless Rotors Using an Actively Controlled Flap and Its Implementation Using Magnetostrictive Actuation," $20^{\text {th }}$ European Rotorcraft Forum, The Netherlands, Amsterdam, pp. $60.1-60.26$.

[43] Prechtl, E. F., Hall, S. R., 2000, "Closed-Loop Vibration Control Experiments on a Rotor with Blade Mounted Actuation," $41^{\text {st }}$ AIAA Structures, Structural Dynamics, and Materials Conference, Atlanta, GA.

[44] Dowson, S. et al., 1995, "Wind Tunnel Test of An Active Flap Rotor - BVI noise and Vibration Reduction," $51^{\text {st }}$ Annual Forum of the American Helicopter Society, Fort Worth, TX, pp. 631-648. 
[45] Roth, D., Enenkl, B., Dietrich, O., "Active rotor control by flaps for vibration reduction - full scale demonstrator and first flight test results", 32nd European Rotorcraft Forum, 12-14 Sep 2006, NLR, Maastricht, the Netherlands, Vol. 1, pp 801-814, 2007.

[46] Wickramasinghe, V. K., and Hagood, N. W., 2005, "Material Characterization of Active Fiber Composite Actuators for Integral Twist-Actuated Rotor Blade Application," Smart Materials and Structures Journal, 13(5), pp. 1155-1165.

[47] Wickramasinghe, V. K., and Hagood, N. W., 2004, "Durability Characterization of Active Fiber Composite Actuators for Helicopter Rotor Blade Applications" Journal of Aircraft, 41(4), pp.931-937

[48] Wilkie, W. K., Bryant, R. G., High, J. W., Fox, R. L., Helbaum, R. F., Jalink, A., Jr., Little, B. D., and Mirick, P. H., "Low-Cost Piezoelectric Actuator for Structural Control Applications," Smart Structures and Materials 2000: Industrial and Commercial Applications of Smart Structures Technologies; Jacobs, J. H., Ed., Proceedings of the SPIE, Vol. 3991, pp. 323-334.

[49] Wickramasinghe, V. K., and Hagood, N. W., 2008, Leading-Edge Materials Science Research, Nova Science Publishers Inc., Hauppauge, New York, Chapter 3 "Experimental Material Characterization of Active Fiber Composite Actuators for Structural Control Applications',' pp. 63-98.

[50] Melnykowycz, M, Kornmann, X., Huber, C., Barbezat, M., and Brunner, A. J., 2006, "Performance of Integrated Active Fiber Composites in Fiber Reinforced Epoxy Laminates," Smart Materials and Structures Journal, 15(1), pp. 204-212.

[51] Wilkie, W. K., Wilbur, M. L., Mirick, P. H., Cesnik, C. E. S., and Shin, S., 1999 "Aeroelastic Analysis of the NASA/ARMY/MIT Active Twist Rotor," 55 th $^{\text {Annual }}$ Forum of the American Helicopter Society, Montreal, Canada, pp. 545-557. 
[52] Wilbur, M. L., Yeager, W. T. Jr., Wilkie, W. K., Cesnik, C. E. S., and Shin, S., 2000, "Hover Testing of the NASA/ARMY/MIT Active Twist Rotor," $56^{\text {th }}$ Annual Forum of the American Helicopter Society, Virginia Beach, VA.

[53] Shin, S.-J. and Cesnik, C. E. S., 2002, "Helicopter Vibration Reduction in Forward Flight using Blade Integral Twist Control," Paper No. 2002-1447, $43^{\text {rd }}$ AIAA Structures, Structural Dynamics, and Materials Conference, Denver, Colorado.

[54] Chen, P. C. and Chopra, I., 1997, "Wind Tunnel Test of a Smart Rotor Model With Individual Blade Twist Control," Journal of Intelligent Material Systems and Structures, 8(5), pp. 414-425.

[55] Rodgers, J. P. and Hagood, N. W., 1998, "Preliminary Mach-scaled Hover Testing of an Integral Twisted-Actuated Rotor Blade," Smart Structures and Materials 1998: Smart Structures and Integrated Systems, Regelbrugge; M. E., Ed. Proceedings of the SPIE, Vol. 3329, pp. 291-308.

[56] Cribbs, R. and Friedmann, P. P., 2000, "Vibration Reduction in Rotorcraft Using an Enhanced ACSR Model," $41^{\text {st }}$ AIAA Structures, Structural Dynamics, and Materials Conference, Atlanta, GA.

[57] Chiu, T. and Friedmann, P. P., 1997, "An Analytical Model for ACSR Approach to Vibration Reduction in a Helicopter Rotor-Flexible Fuselage System," The Aeronautical Journal, 101(1009), pp. 399-408.

[58] Staple, A. E., 1990, “An Evaluation of Active Control of Structural Response and a Means of Reducing Helicopter Vibration," $46^{\text {th }}$ Annual Forum of the American Helicopter Society, Washington DC, pp. 3-17.

[59] Welsh, W. A., Fredrickson, C., Rauch, C., and Lyndon, I., 1995, "Flight Test of an Active Vibration Control System on the UH-60 Black Hawk Helicopter," $51^{\text {st }}$ Annual Forum of the American Helicopter Society, Fort Worth, TX, pp. 393-400.

[60] Welsh, W. A., Von Hardenberg, P. C., Von Hardenberg, P. W., and Staple, A. E., 1990, "Test and Evaluation of Fuselage Vibration Utilizing Active Control of 
Structural Response (ACSR) Optimized to ADS-27," $46^{\text {th }}$ Annual Forum of the American Helicopter Society, Washington DC, pp. 21-37.

[61] Hackett, N., 1995, "EH101 Active Control of Structural Response Development, Productionisation and Certification," EH101 Avionics- Civil and Military Conference, London, United Kingdom, pp. 5.1-5.10.

[62] Goodman, R. K. and Millott, T. A., 2000, "Design, Development, and Flight Testing of the Active Vibration Control System for the Sikorsky S-92," $56^{\text {th }}$ Annual Forum of the American Helicopter Society, Virginia Beach, VA.

[63] Man P.D., Lemerle P. and Verschueren J-P. et al., 2005, "An Investigation of A Semi-Active Suspension for A Fork Lift Truck", Vehicle System Dynamics, 43(2), pp.107-119.

[64] Cai L. and Chen D., 2004, "A Two-Stage Vibration Isolation System Featuring An Elctroerheological Damper via the Semi-active Static Output Feedback Variable Structure Control Method", Journal of Vibration and Control, 10(5), pp. 683-706.

[65] Choi, S. B., Choi, J. H., Nam, M. H., Cheong, C. C., and Lee, H. G., 1999, “A SemiActive Suspension Using ER Fluids for a Commercial Vehicle Seat," Journal of Intelligent Material Systems and Structures, 9(8), pp. 601-606.

[66] Choi, S. B., Nam, M. H., and Lee, B. K., 2000, "Vibration Control of MR Seat Damper for Commercial Vehicles," Journal of Intelligent Material Systems and Structures, 11(12), pp. 936-944.

[67] Wu, X., and Griffin, M. J., 1997, “A Semi-Active Control Policy to Reduce the Occurrence and Severity of End-Stop Impacts In A Suspension Seat with an Electrorheological Fluid Damper," Journal of Sound and Vibration, 203(5), pp. 781793.

[68] Choi, Y. T., and Wereley, N. M., 2005, "Biodynamic Response Mitigation to Shock Loads Using Magnetorheological Helicopter Crew Seat Suspensions," Journal of Aircraft, 42(5), pp. 1288-1295. 
[69] Hiemenz G. J., Hu W. and Wereley N., 2008, “Semi-Active Magnetorheological Helicopter Crew Seat Suspension for Vibration Isolation", Journal of Aircraft, 45(3), pp. 945-953.

[70] Hiemenz, G. J., Hu, W. and Wereley, N. M., 2008, "Semi-Active Magnetorheological Helicopter Crew Seat Suspension for Vibration Isolation," AIAA Journal of Aircraft, 45(3), pp. 945-953.

[71] Wickramasinghe, V. K., Yong, C., and Zimcik, D.G., 2010, "Development of an Active Suspension System for Adaptive Vibration Control of Helicopter Seats," NATO RTO AVT-170/RSM-025 on Active Suspension Technologies for Military Vehicles and Platforms, Sofia, Bulgaria.

[72] Yong, C., Wickramasinghe, V. K., Corbin, A., and Zimcik, D.G., 2010, "Investigation of Helicopter Seat Structural Dynamics for Aircrew Vibration Mitigation," $66^{\text {th }}$ Annual International Forum of the American Helicopter Society, Phoenix, AZ.

[73] Wickramasinghe, V., Chen, Y., and Zimcik, D., 2009, "Development of Adaptive Helicopter Seat for Aircrew Vibration Reduction," $20^{\text {th }}$ International Conference of Adaptive Structures and Technologies (ICAST 2009), Hong Kong.

[74] Chen, Y., Wickramasinghe, V., and Zimcik, D., 2009, "Development of Smart Structure Systems for Aerospace Structural Vibration and Noise Control Applications," $2^{\text {nd }}$ International Conference on Smart Materials and Nanotechnology in Engineering, WeiHai, China.

[75] Chen, Y., Wickramasinghe, V., and Zimcik, D., 2008, "Development of Adaptive Helicopter Seat Systems for Aircrew Vibration Mitigation", Smart Structures and Materials 2008: Active and Passive Smart Structures and Integrated Systems, Proceedings of the SPIE, Vol. 6928, San Diego, CA. 
[76] Chen, Y., Wickramasinghe, V., and Zimcik, D., 2011, "Development of Adaptive Helicopter Seat for Aircrew Vibration Reduction," Journal of the Intelligent Material Systems and Structures, 22(5), pp. 489-502.

[77] Wickramasinghe, V. Chen, Y., and Zimcik, D., 2009, "Development of Adaptive Seat Mount for Helicopter Pilot Vibration Reduction ", Journal of Vibration and Control, 15(12), pp. 1809-1825.

[78] Chen, Y., Wickramasinghe, V. K., and Zimcik, D. G., 2007, "Development of Smart Structure Systems for Helicopter Vibration and Noise Control", Transactions of the Canadian Society for Mechanical Engineering, 31(1), pp. 39-56.

[79] Chen, Y. Wickramasinghe, V. and Zimcik, D., 2011, "Investigation of Helicopter Seat Structural Dynamics for Aircrew Vibration Mitigation," Journal of the American Helicopter Society, 56(1), pp. 12006-1-12006-10.

[80] Harrison, M.F., Neary, J.P., Albert, W.J., and Croll, J.C., 2011, "Neck Pain and Muscle Function in a Population of CH-146 Helicopter Aircrew," Aviation Space and Environmental Medicine, 82 (12), pp. 1125-1130.

[81] Ulker, F. D., and Nitzsche, F., 2012, "Novel Controller Design Demonstration for Vibration Alleviation of Helicopter Rotor Blades" Active and Passive Smart Structures and Integrated Systems 2012, Sodano, H. A., Ed., Proceedings of the SPIE, Vol. 8341, San Diego, CA.

[82] Sahin, M., and Aridogan, U., 201 1, "Performance Evaluation of Piezoelectric sensor/actuator on Active Vibration Control of a Smart Beam." Institution of Mechanical Engineers Part I: Journal of Systems and Control Engineering, 225(5), pp. 533-547.

[83] Shen, G., Zheng, S.-T., Ye, Z.-M., Huang, Q.-T., Cong, D.-C., Han, J.-W., 2011, "Adaptive Inverse Control of Time Waveform Replication for Electrohydraulic Shaking Table," Journal of Vibration and Control, 17(11), pp. 1611-1633. 
[84] Ganguli, R., 2010, "Rotorcraft Research in India: Recent Developments," Aircraft Engineering and Aerospace Technology, 82(5), pp. 305-319.

[85] VerticalNews, "Findings from National Research Council in Helicopters Reported," Accessed 26 June 2011, http://www.verticalnews.com/premium_newsletters /Transportation-Business-Journal/201 1-06-26/66072TBJ.html

[86] United States Department of Defense, 2000, "Test Method Standard for Environmental Engineering Considerations and Laboratory Tests", MIL-STD-810F.

[87] Johnson, W., 1994, Helicopter Theory, Dover Publications Inc., New York, NY.

[88] Wickramasinghe V. and Chen Y., 2008, "Analysis of Vibration on Helicopter Aircrew Based on IAR Bell 412 Flight Tests and Shaker Tests on the Seat Structure", LTR-SMPL-2008-0068, National Research Council Canada, Ottawa, Canada.

[89] Bell 412EP Product Specifications, Accessed 24 January 2012, Bell Helicopter, http://www.bellhelicopter.com/en_US/Commercial/Bell412/1291148331974.html

[90] LMS Instruments, Accessed 16 July 2012, LMS SCADAS III Data Acquisition Front-End, Breda, The Netherlands, http://www.Imsintl.com.

[91] LMS International, Accessed 16 July 2012, LMS Test.Lab Spectral Testing Rev 10B, Leuven, Belgium, http://www.lmsintl.com.

[92] International Standards Organization, 1997, "Mechanical Vibration and ShockEvaluation of Human Exposure to Whole-Body Vibration, Part 1: General requirements," ISO 2631-1, Geneva, Switzerland.

[93] Griffin, M.J., 1990, Handbook of Human Vibration, Academic Press, London, UK.

[94] Woodson, W.E., Tillman, B., Tillman, P., 1992, Human Factors Design Handbook, $2^{\text {nd }}$ Ed., McGraw-Hill Book Company, New York, NY.

[95] Salyards, K. A., Hanagan, L. M., Trethewey, M. W., 2006, "Comparing Vibration Serviceability Assessment Measures For Stadium Rock Concert Data," XXIV 
International Modal Analysis Conference, Society for Experimental Mechanics, Bethel, CT.

[96] Ellis, B.R. and Littler, J.D., 2004, "Response of Cantilever Grandstands to Crowd Loads. Part 1: Serviceability Evaluation," Proceedings of Institution of Civil Engineers: Structures and Buildings, 157(4), pp.235-241.

[97] Gillespie, T.D., 1992, Fundamentals of Vehicle Dynamics, SAE Publishers, Warrendale, PA.

[98] Chen Y., Wickramasinghe V. and Zimcik D.G., 2009, "Development of Adaptive Seat Mounts for Helicopter Aircrew Body Vibration Reduction," Journal of Vibration and Control, 15(12), pp. 1809-1825.

[99] Ewins, D. J., 1984, Modal Testing: Theory and Practice, Research Studies Press Ltd., New York, NY.

[100] Craig, R., 1981, Structural Dynamics: An Introduction to Computer Methods, John Wiley \& Sons, New York, NY.

[101] Larsen, G.C., Hansen, M. H., Baumgart, A., Carlén, I, 2002, "Modal Analysis of Wind Turbine Blades, Ris $ø-R-1181(\mathrm{EN})$, Ris $\varnothing$ National Laboratory, Roskilde, Denmark.

[102] Heylen, W., Lammens, S., Sas, P., 1995, Modal Analysis Theory and Testing, Department of Mechanical Engineering, KULeuven, Belgium.

[103] Maia, N., Silva, J., 1997, Theoretical and Experimental Modal Analysis, Research Studies Press, Taunton, UK.

[104] LMS Instruments, Accessed 16 July 2012, LMS SCADAS III Data Acquisition Front-End, Breda, The Netherlands, http://www.lmsintl.com.

[105] LMS International, Accessed 16 July 2012, LMS Test.Lab - Random Control Software Module Rev 10B, Leuven, Belgium, http://www.lmsintl.com. 
[106] Peeters, B., Van der Auweraer, H., Guillaume, P., Leuridan, J., 2004, “The PolyMAX Frequency-Domain Method: A New Standard for Modal Parameter Estimation?" Shock and Vibration, 11(3), pp.395-409.

[107] Mevel, L., Benveniste, A., Basseville, M., Goursat, M., Peeters, B., Van der Auweraer H., and Vecchio, A., 2006, "Input/Output Versus Output-Only Data Processing for Structural Identification-Application to In-Flight Data Analysis" Journal of Sound and Vibration, 295(3-5), pp. 531-552.

[108] Phillips, A.W., Allemang, R.J., 2004, “A Low Order Implementation of the Polyreference Least Squares Complex Frequency (LSCF) Algorithm", ISMA International Conference on Noise and Vibration Engineering, Katholieke Universiteit Leuven, Belgium.

[109] Guillaume P., P. Verboven, S. Vanlandiut, H. Van der Auwaerer and Peeters, B., 2003, "A Poly-Reference Implementation of the Least-Squares Complex FrequencyDomain Estimator," $21^{\text {st }}$ International Modal Analysis Conference, Kissimmee, FL.

[110] Cooper, J. E., 1990, "Comparison of Modal Parameter Estimation Techniques on Aircraft Structural Data" Mechanical Systems and Signal Processing, 4(2), pp. 157172.

[111] Wan Y., and Schimmels, J.M., 1995, “A Simple Model that Captures the Essential Dynamics of A Seated Human Exposed to Whole Body Vibration" Advances in Bioengineering, ASME, 31, pp. 333-334.

[112] Liang, C.-C., Chiang, C.-F., 2008, "Modeling of a Seated Human Body Exposed to Vertical Vibrations in Various Automotive Postures", Industrial Health, 46, pp. 125-137, 2008.

[113] Liang, C.-C., Chiang, C.-F., 2006, “A Study On Biodynamic Models of Seated Human Subjects Exposed to Vertical Vibration", International Journal of Industrial Ergonomics, 36(10), pp. 869-890. 
[114]Kitazaki S. and Griffin M.J., 1997, "A Modal Analysis of Whole Body Vertical Vibration, using a Finite Element Method of the Human Body," Journal of Sound and Vibration, 200(1), pp. 83-103.

[115]Matsumoto, Y., Griffin, M. J., 1998, "Movement of Upper-Body of Seated Subjects Exposed to Vertical Whole-Body Vibration at the Principle Resonance Frequency," Journal of Sound and Vibration, 215(4), pp. 743-762.

[116] Rivin, E. L., 2003, Passive Vibration Isolation, The American Society of Mechanical Engineers, New York, NY.

[117] Jaffe, B., Cook, W. R., Jaffe, H., 1971, Piezoelectric Ceramics, Academic Press; New York, NY.

[118] Garg, D. P., and Anderson, G. L., 2003, "Structural Damping and Vibration Control via Smart Sensors and Actuators," Journal of Vibration and Control, 9(12), pp. 1421-1452.

[119] Nelson, L. J., 2002, "Literature Review: Smart Piezoelectric Fibre Composites," Materials Science and Technology, 18(11), pp. 1245-1256.

[120] Morgan Matroc Inc., 2005, "Piezoceramic Data Sheets," Morgan Matroc Electro Ceramic Division, Bedford $\mathrm{OH}$.

[121] Giurgiutiu V., 2000, "Recent Advances in Smart Material Rotor Control Actuation," $41^{\text {st }}$ AIAA Structures, Structural Dynamics, and Materials Conference, Atlanta, GA.

[122] Wirnitzer, J., Kistner, A., and Gaul, L., 2002, "Optimal Placement of Semi-Active Joints in Large Space Truss Structures," Smart Structures and Materials 2002:

Damping and Isolation, Agnes; G. S., Ed., Proceedings of the SPIE, Vol. 4697, pp. 246-257.

[123] Yan, Y. J., and Yam, L. H., 2002, “Optimal Design of Number and Locations of Actuators in Active Vibration Control of a Space Truss," Journal of Smart Materials and Structures, 11(4), pp. 496-503. 
[124] Hall S. R., Tzianetopoulou T., Straub, F. K., and Ngo, H. "Design and Testing of a Double X-Frame Piezoelectric Actuator," Smart Structures and Materials 2000: Smart Structures and Integrated Systems, Wereley; N. M., Ed., Proceedings of the SPIE, Vol. 3985, pp. 26-37.

[125] Prechtl, E. F. and Hall, S. R., 2000, "Closed-loop Vibration Control Experiments on a Rotor With Blade Mounted Actuation," $41^{s t}$ AIAA Structures, Structural Dynamics, and Materials Conference and Exhibit, Atlanta, GA.

[126] Straub, F. K., Ngo, H. T., Anand, V., and Domzalski, D. B., 2000, "Development of a Piezoelectric Actuator for Trailing edge Flap Control of Full-Scale Rotor Blades," Smart Materials and Structures Journal, 10(1), pp. 25-34.

[127] Sutton, T. J., Elliott, S. J., Brennan, M. J., Heron, K. H., and Jessop, D. A. C., 1997, "Active Isolation of Multiple Structural Waves on a Helicopter Gearbox Support Strut," Journal of Sound and Vibration, 205(1), pp. 81-101.

[128] Li, M., Lim, T. C., Shepard, S. W., and Guan, Y. H., 2005, "Experimental Active Vibration Control of Gear Mesh Harmonics in a Power Recirculation Gearbox System using a Piezoelectric Stack Actuator," Journal of Smart Materials and Structures, 14(5), pp. 917-927.

[129] Morgan, D. R., and Sanford, C., 1992, "A Control Theory Approach to the Stability and Transient Analysis of the Filtered-x LMS Adaptive Notch Filter," IEEE Transactions on Signal Processing, 40(9), pp. 2341-2346.

[130] Chen Y., Zimcik D. G. and Wickramasinghe V.K., 2005, "An Adaptive Impedance Control Algorithm for Helicopter Blade Vibration Suppression," Journal of Vibration and Control, 11(4), pp. 543-560.

[131] Chen Y., Wickramasinghe, V. and Zimcik, D. G., 2009, "Development of Adaptive Seat Mounts for Helicopter Aircrew Body Vibration Reduction," Journal of Vibration and Control, 15(12), pp. 1809-1825. 
[132] Glover J. R., 1997, “Adaptive Noise Canceling Applied to Sinusoidal Interferences," IEEE Transactions on Acoustics, Speech, and Signal Processing, 25(6), pp. 484-491.

[133] Eriksson L J, Allie M. A., 1989, "Use of Random Noise for Online Transducer Estimate in an Adaptive Active Attenuation System", Journal of the Acoustical Society of America, 85(2), pp. 797-802.

[134] Widrow, B., and Stearns, S. D., 1985, Adaptive Signal Processing, Prentice-Hall, Englewood Cliffs, NJ.

[135] Hansen, C. H., and Snyder, S. D., 1997, Active Control of Noise and Vibration, (E \& FN SPON, New York, NY.

[136] Elliott, S. J., and Nelson, P. A., 1989, "Multiple-point equalization in a room using adaptive digital filters," Journal of Audio Engineering Society, 37(11), pp. 899-907.

[137] Gawronski, W., 2004, Advanced Structural Dynamics, and Active Control of Structures, Springer, New York, NY.

[138] Rivin, E.I., 2003, Passive Vibration Isolation, ASME Press, New York, NY.

[139] United States Department of Defense, 1996, "Military Specifications: Seat System, Aircrew, Crash Resistant; General Specification," Military Standard MIL-S81771 A.

[140] Fairley, T.E. and Griffin, M.J., 1986, "A Test Method for the Prediction of Seat Transmissibility," SAE Paper 860046, Society of Automotive Engineers, International Congress and Exposition, Detroit, MI.

[141] Colvin, D.P. D.R. Myers, C. Potter, D. Moody, C.J. Lord and A.L. Patra, 1993, "HACS - A New Cushioning Structure for Impact Protection," ASME Advances in Bioengineering, Vol. 26. 
[142] Colvin, D.P., McKinney, R. A., 2005, "Reduction in Body Vibration and Spinal Impact with HACS Cushioning," ASME International Mechanical Engineering Congress and Exposition, IMECE2005-82911, Orlando, FL.

[143] Colvin, D.P. and Potter, C. S., 1991, "Cushioning Structure," US. Patent $5,030,501$.

[144] Colvin, D.P. and Potter, C. S., 1996, "Cushioning Structure," US Patent $5,518,802$.

[145] Cinats J., Reid D.C., Haddow J.B., 1987, "A Biomechanical Evaluation of Sorbothane," Clinical Orthopaedics and Related Research, 222, pp. 281-288.

[146] Schueneman, H. H., 1988, "A Comparison of Three Different Cushion Test Methods," Current Trends in Protective Packaging of Computers and Electronic Components, ASTM STP 994, Robert J. Peache and Denis O'Sullivan, Eds. American Society for Testing and Materials, PA, pp. 58-64.

[147] Rodríguez, E. E. and Gesnouin, G. A., 2007, "Effective Mass of an Oscillating Spring," The Physics Teacher, 45(2), pp. 100-103. 